Prepared in cooperation with the

KANSAS WATER OFFICE

\title{
Effects of Irrigation Practices on Water Use in the Groundwater Management Districts Within the Kansas High Plains, 1991-2003
}

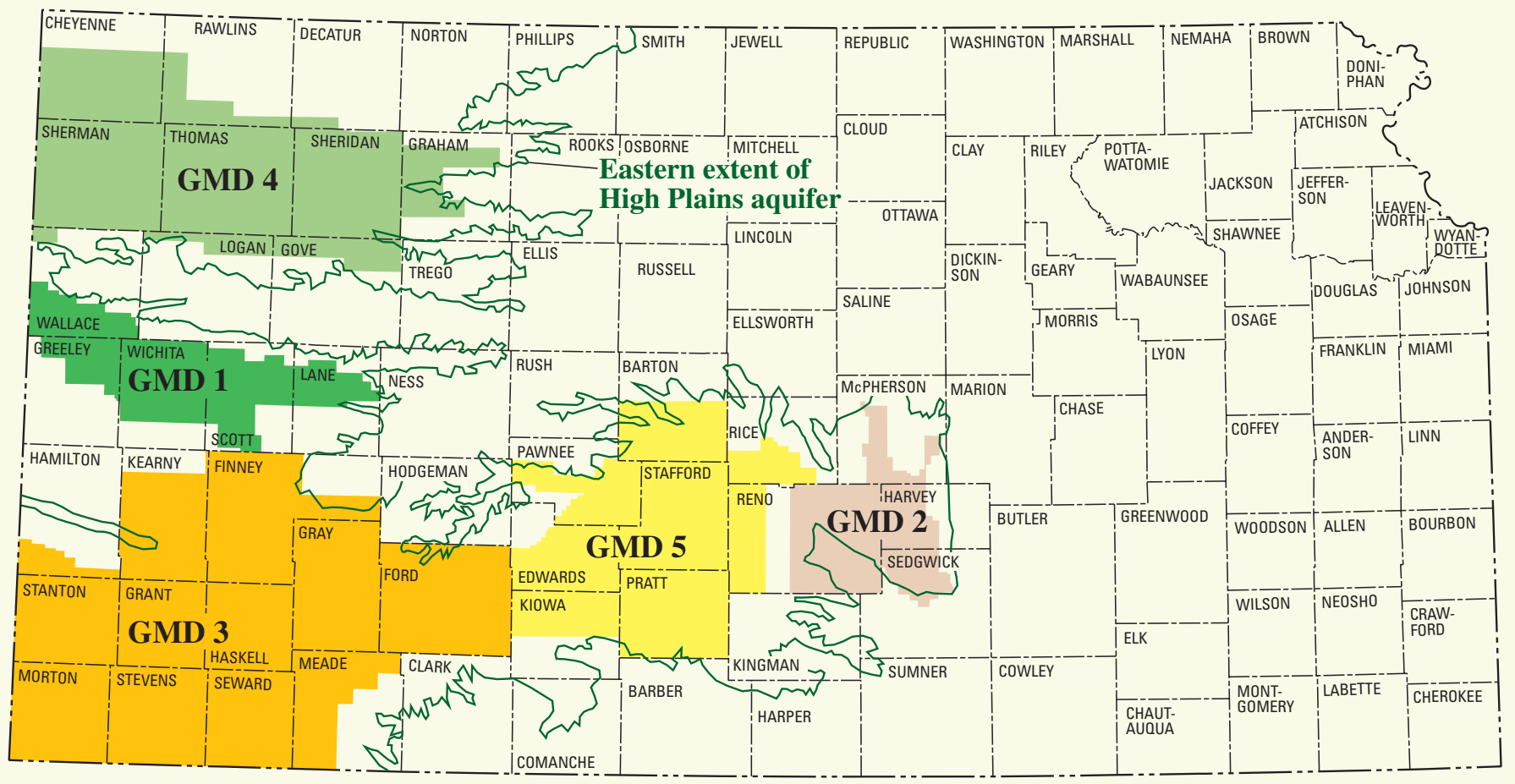

Scientific Investigations Report 2006-5069 


\section{Effects of Irrigation Practices on Water Use in the Groundwater Management Districts Within the Kansas High Plains, 1991-2003}

By Charles A. Perry

Prepared in cooperation with the Kansas Water Office

Scientific Investigations Report 2006-5069 


\title{
U.S. Department of the Interior Gale A. Norton, Secretary
}

\author{
U.S. Geological Survey \\ P. Patrick Leahy, Acting Director
}

\section{U.S. Geological Survey, Reston, Virginia: 2006}

\author{
For sale by U.S. Geological Survey, Information Services \\ Box 25286, Denver Federal Center \\ Denver, CO 80225 \\ For more information about the USGS and its products: \\ Telephone: 1-888-ASK-USGS \\ World Wide Web: http://www.usgs.gov/
}

Any use of trade, product, or firm names in this publication is for descriptive purposes only and does not imply endorsement by the U.S. Government.

Although this report is in the public domain, permission must be secured from the individual copyright owners to reproduce any copyrighted materials contained within this report.

Suggested citation:

Perry, C.A., 2006, Effect of irrigation practices on water use in the Groundwater Management Districts within the Kansas HIgh Plains, 1991-2003: U.S. Geological Survey Scientific Investigations Report 2006-5069, 93 p. 


\section{Contents}

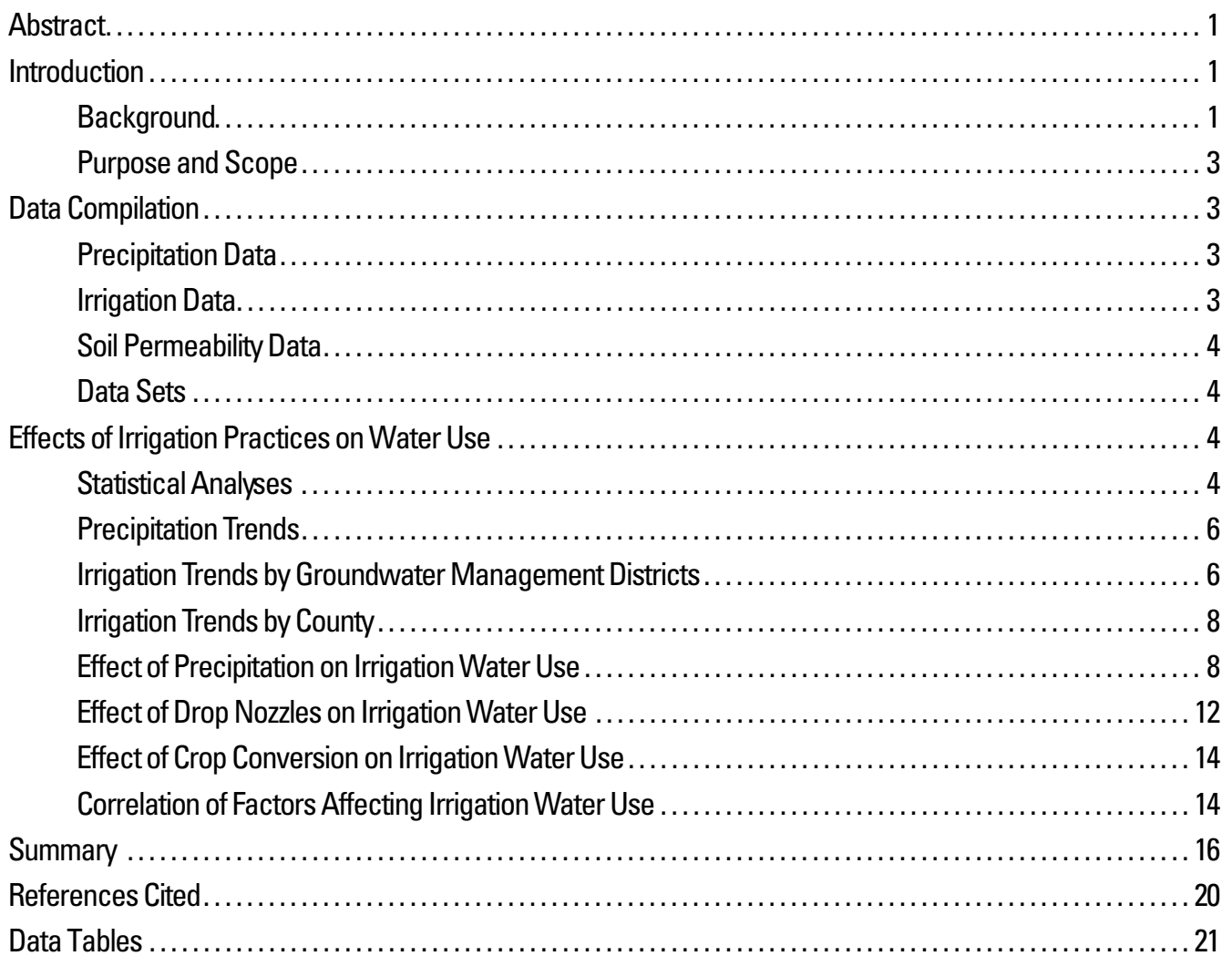

\section{Figures}

1. Map showing location of High Plains aquifer, counties included in study, Groundwater Management

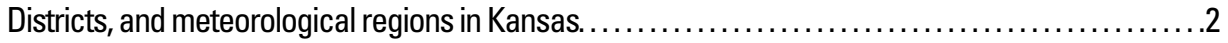

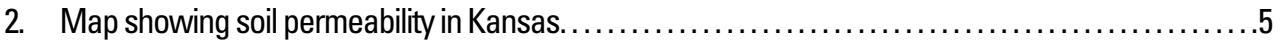

3-12. Graphs showing:

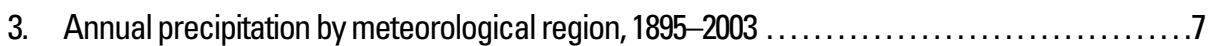

4. Relation between irrigation water use for Groundwater Management Districts and average precipitation for meteorological regions $1,4,7$, and $8,1991-2003 \ldots \ldots \ldots \ldots \ldots \ldots \ldots . \ldots$

5. Irrigation water use and annual regional precipitation by Groundwater Management District,

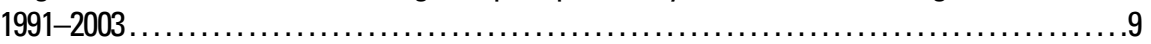

6. Acres irrigated by system type for Groundwater Management Districts, 1991-2003 ....... 11

7. Acres irrigated by crop type for Groundwater Management Districts, 1991-2003......... 13

8. Relation between irrigation water use and precipitation, $1991-2003 \ldots \ldots \ldots \ldots \ldots \ldots \ldots \ldots$

9. Comparison of measured irrigation water use and precipitation-adjusted irrigation water use for Groundwater Management Districts using two different equations for adjustment..... 15

10. Average precipitation plus irrigation-applied water for all Groundwater Management Districts,

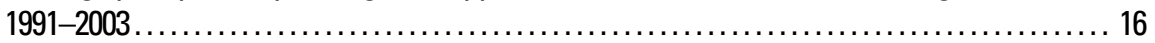

11. Relation between irrigation application rates for center pivot systems with and without drop nozzles for Groundwater Management Districts, 1992-2003....................... 17 
12. Relation between irrigation application rates for flood irrigation systems and center pivot systems with drop nozzles for Groundwater Management Districts, 1992-2003

\section{Tables}

1. Precipitation, irrigation water use, acres irrigated, irrigation application rate by system type, and soil permeability for counties in the Groundwater Management Districts within the Kansas High Plains, 1991-2003. 22

2. Irrigation water use, acres irrigated, and irrigation application rate by crop type for counties in the Groundwater Management Districts within the Kansas High Plains, 1991-2003 . .............. 42

3. Irrigation water use, acres irrigated, and irrigation application rate by system type for the Groundwater Management Districts within the Kansas High Plains, 1991-2003.

4. Irrigation water use, acres irrigated, and irrigation application rate by crop type for the Groundwater Management Districts within the Kansas High Plains, 1991-2003.

5. Average regional precipitation and irrigation water use in the Groundwater Management Districts

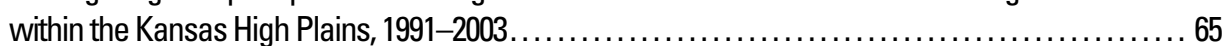

6. Trends in precipitation by meteorological regions in Kansas, $1895-2003 \ldots \ldots \ldots \ldots \ldots \ldots \ldots \ldots . \ldots \ldots$

7-12. Trends in precipitation and irrigation by system type, crop type, and prorated crop type for:

7. Groundwater Management District 1, west-central Kansas, 1991-2003 ...................69 69

8. Groundwater Management District 2, south-central Kansas, 1991-2003 . ............... 72

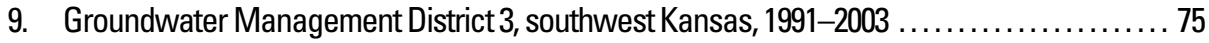

10. Groundwater Management District 4, northwest Kansas, 1991-2003.................. 78

11. Groundwater Management District5, south-central Kansas, 1991-2003 . ................ 81

12. All Groundwater Management Districts in Kansas, 1991-2003 .......................... 84

13. Results of regression analysis for irrigation water use and irrigation application rates based on

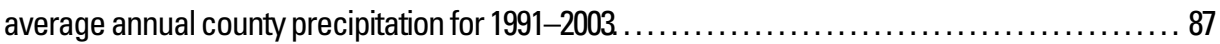

14. Results of regression analysis for irrigation water use based on average annual precipitation by

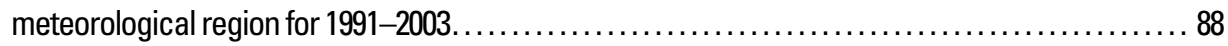

15. Irrigation water use adjusted using average regional precipitation by meteorological region for Groundwater Management Districts within the Kansas High Plains, 1991-2003 ............... 89

16. Average precipitation plus irrigation-applied water for all Groundwater Management Districts within

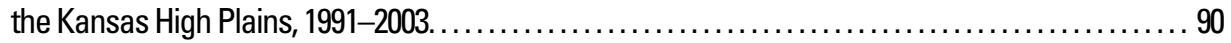

17. Relation between irrigation system types and application rates, 1992-2003, for Groundwater

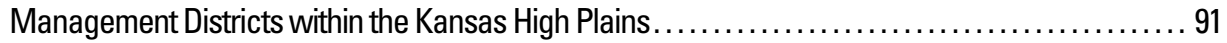

18. Correlation coefficients for irrigation water-use factors for 33 counties in the Groundwater Management Districts within the Kansas High Plains, $1991-2003 \ldots \ldots \ldots \ldots \ldots \ldots \ldots \ldots \ldots . \ldots 2$

19. Predictive equations for irrigation water use and irrigation application rates from county data for all Groundwater Management Districts within the Kansas High Plains, 1991-2003 .... 


\section{Conversion Factors, Abbreviations, and Datum}

\begin{tabular}{lcl}
\hline Multiply & By & To obtain \\
\hline & Length & \\
inch (in.) & 2.54 & centimeter $(\mathrm{cm})$ \\
mile (mi) & 1.609 & kilometer $(\mathrm{km})$ \\
& Area & \\
acre & 4,047 & square meter $\left(\mathrm{m}^{2}\right)$ \\
acre & 0.4047 & hectare $(\mathrm{ha})$ \\
acre & 0.4047 & square hectometer $\left(\mathrm{hm}^{2}\right)$ \\
acre & 0.004047 & square kilometer $\left(\mathrm{km}^{2}\right)$ \\
acre per year (acre/yr) & 4,047 & square meter per year $\left(\mathrm{m}^{2} / \mathrm{yr}\right)$ \\
& Volume & \\
acre-foot (acre-ft) & 1,233 & cubic meter $\left(\mathrm{m}^{3}\right)$ \\
acre-foot (acre-ft) & 0.001233 & cubic hectometer $\left(\mathrm{hm}{ }^{3}\right)$ \\
& Rate & \\
acre-foot per year (acre-ft/yr) & 1,233 & cubic meter per year $\left(\mathrm{m}^{3} / \mathrm{yr}\right)$ \\
acre-foot per acre (acre-ft/acre) & 0.3048 & cubic meter per square $\mathrm{meter}\left(\mathrm{m}^{3} / \mathrm{m}^{2}\right)$ \\
inch per hour (in/h) & 2.54 & centimeter per hour $\left(\mathrm{cm}^{2} / \mathrm{hr}\right)$ \\
\hline
\end{tabular}

Horizontal coordinate information is referenced to the North American Datum of 1983 (NAD 83). 


\title{
Effect of Irrigation Practices on Water Use in the Groundwater Management Districts Within the Kansas High Plains, 1991-2003
}

\author{
By Charles A. Perry
}

\section{Abstract}

Data compiled for the High Plains region of Kansas that includes five Groundwater Management Districts (GMDs) were analyzed for trends in irrigation water use, acres irrigated, precipitation, irrigation system types, and irrigated crop types to determine the effects of irrigation practices on water use over time. For the study period 1991 through 2003, precipitation decreased significantly (with 95-percent confidence) in northwestern and west-central Kansas but not in the southwestern and south-central parts of the State. Irrigation water use had no statistically significant trend during this period. There was a $\operatorname{good}(\mathrm{R}=-0.77)$ relation between average regional precipitation and total GMD irrigation water use. When irrigation water use was adjusted for this relation, there was a positive trend (90-percent confidence level) in the adjusted irrigation water use. Another adjustment to water use was made using the ratio of annual precipitation to 1991-2005 average precipitation, which resulted in a negative trend (95-percent confidence level) in irrigation water use. This demonstrated the contradictory nature of precipitation adjustments to water use, making their utility somewhat suspect. GMD 3 in southwestern Kansas used 63 percent of the total acre-feet of irrigation water within all the GMDs.

When all GMDs are considered, the number of irrigated acres for flood and center pivot systems without drop nozzles decreased significantly during the study period. At the same time the number of drop nozzle irrigated acres increased significantly. The number of irrigated acres of water-intensive crops (corn, alfalfa, and soybeans) also increased significantly, whereas the number of less- or non-water-intensive crops (grain sorghum and wheat), and multiple crop type acres decreased. Drop nozzle irrigation systems used approximately 2 percent less water in a year-by-year comparison than center pivot systems and 8 to 11 percent less water than flood irrigation.

The best estimator of irrigation water use incorporated total acres irrigated and annual average or March-October regional precipitation. A conclusion that can be drawn from the trend analyses described in this report is that, although irrigation water use for all GMDs showed no statistically significant trend, an apparent increased efficiency of center pivots irrigation systems with drop nozzles has allowed more waterintensive crops to be grown on more irrigated acres.

\section{Introduction}

\section{Background}

The High Plains aquifer underlies parts of Colorado, Kansas, Nebraska, New Mexico, Oklahoma, South Dakota, Texas, and Wyoming (fig. 1). Approximately 90 percent of the total water used for irrigation in Kansas in 2003 was pumped from wells in counties underlain by the High Plains aquifer. Historically, the saturated thickness of the High Plains aquifer has been declining in Kansas since the 1940s (McGuire and Sharpe, 1997). These declines have been mitigated somewhat by implementing various conservation strategies such as local regulation, changes in irrigation management practices, and significant advances to improve the efficiency of irrigation systems (McGuire and Sharpe, 1997).

In an effort to reduce consumptive use of ground water in western Kansas, State government has promoted various programs such as cost sharing for more efficient irrigation systems, assistance with irrigation scheduling, and preparation of conservation plans. These efforts have been ongoing for more than 10 years, during which time State and Federal agencies have invested substantially in equipment cost sharing and technical assistance to irrigators. Cost sharing is implemented locally through the county conservation districts. In State fiscal year 2003, Kansas conservation districts eligible for irrigation initiative allocations received approximately $\$ 388,000$ to implement irrigation efficiency practices (Kansas State Conservation Commission, 2005).

Five Groundwater Management Districts (GMDs) were created in the 1970s in the western and south-central parts of the State. GMDs provide water-use administration, planning, and information. The districts are governed by local boards and have been instrumental in providing information and identifying research and regulatory needs within their boundaries. 


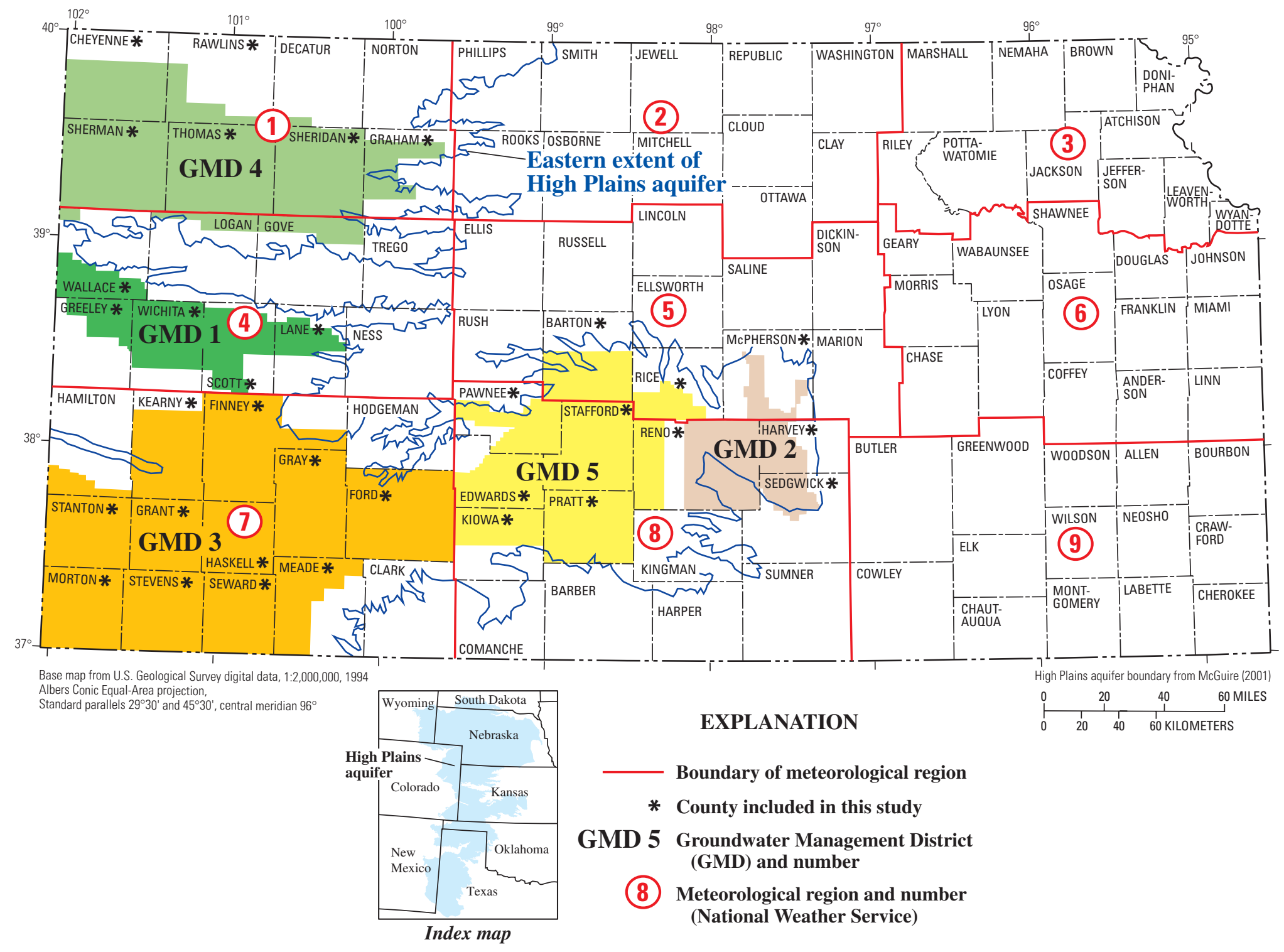

Figure 1. Location of High Plains aquifer, counties included in study, Groundwater Management Districts, and meteorological regions in Kansas. 
There is substantial interest from State water agencies, GMDs, and irrigators in evaluating the effectiveness of the costsharing investments in actually reducing consumptive irrigation water use. There is anecdotal evidence in some areas of the State that improved irrigation system efficiency has led to a net increase in water use as a result of either increases in the number of acres irrigated, increases in the length of the irrigation season by growing multiple crops, or irrigating crops with greater water requirements. This 1-year study by the U.S. Geological Survey (USGS), done in cooperation with the Kansas Water Office, was conducted to determine the effect of more efficient irrigation systems on irrigation water use in areas underlain by the High Plains aquifer in Kansas.

There are many factors that affect irrigation water use including precipitation, temperature, wind, number of acres irrigated, soil permeability, crop types, crop application rates, length of irrigation season, and irrigation system type. The question of whether water use has decreased or not as a result of the addition of cost-shared drop nozzles to center pivot irrigation systems needed to be addressed.

\section{Purpose and Scope}

The purpose of this report is to describe changes in irrigation water use during the past 13 years (1991-2003) in areas of Kansas in proximity of the High Plains aquifer and to evaluate the effect of more efficient irrigation systems on water use.

Relations between irrigation water use and changes in precipitation, acreage, crop type, and system type are described using existing county data. Irrigation water use data in this report primarily but not exclusively represent withdrawals from the High Plains aquifer.

The study area was limited to 33 counties, of which at least one-third or more of the county lies within one of the five GMDs (fig. 1). Reported water-use data from 1991 to 2003 (Kansas Water Office and Kansas Department of Agriculture, 1993-2005), including acre-feet applied, acres irrigated, crop type, and irrigation system type, along with the climatic variable precipitation were analyzed statistically. Site-specific data reported by irrigators each year to the Kansas Department of Agriculture, Division of Water Resources, and aggregated by county were used in this investigation to indicate any significant changes over time in Kansas irrigation water use, the geographic distribution of any such changes, and whether these changes in water use may be related to irrigation practices. The 1991 start year was chosen because that was the first year the State collected data on irrigation system type. Summaries of county irrigation withdrawals by method of reporting, acres irrigated by crop type, and acres irrigated by system type were prepared for each year from 1991 through 2003. These data were used to develop annual county-level estimates for irrigation water use, number of acres irrigated and acre-foot per acre application rates, using flood, center pivot with and without drop nozzles, or other systems, and number of acres planted to alfalfa, corn, grain sorghum, soybeans, wheat, and multiple crop types. Additional data considered in this investigation included precipitation from weather stations monitored by the U.S. Weather Service throughout western and south-central Kansas.

Statistical analyses of the data were conducted with simple procedures such as trend analyses and multiple least-squares regression to identify significant variables that affect irrigation water use. Statistical relations between irrigation water use and precipitation, crop types, and irrigation system type were evaluated and interpreted. County and GMD tables showing changes in irrigation water use and percentage of drop nozzle systems were generated and compared (see tables 1-19 in section on "Data Tables" at the back of this report).

The scope of this report did not include computing actual consumptive water use. Consumptive water use is difficult to measure even on a highly instrumented individual test site. Efficient irrigation systems reduce both evaporation and deep percolation. The rate of deep percolation of applied water returning to the aquifer can only be estimated. However, if the assumption that a certain percentage of applied water finds its way back to the water table under nonefficient irrigation systems, a lesser percentage of water would return to the aquifer under more efficient irrigation systems. More efficient systems would imply that less water would return to the aquifer.

\section{Data Compilation}

\section{Precipitation Data}

Precipitation data used in this study have been collected by the National Oceanic and Atmospheric Administration (18952004) since 1895. Average monthly values for 1895-2004 for the nine meteorological regions in Kansas (fig. 1) were compiled by the National Oceanic and Atmospheric Administration's National Climatic Center in Asheville, North Carolina. These precipitation data also have been aggregated into average monthly values for each county in Kansas and are available through the Kansas State University Weather Data Library (http://www.oznet.ksu.edu/wdl/) for the period 1981 through 2004. Monthly precipitation values were used to compute a seasonal (March through October) precipitation total for each county for the time period 1991 through 2003 (table 1 at the back of this report).

\section{Irrigation Data}

Irrigation data for this study have been collected by the Kansas Department of Agriculture, Division of Water Resources, on an annual basis since 1989. Annual water-use reports are required of all irrigators in Kansas, and return rates of the reports to the Kansas Department of Agriculture are about 99 percent as a result of penalties for nonreporting (Statutory Authority: K.S.A. 82a-701 et seg., K.S.A. 82a-1501 through 
1508, as amended) (http://www.kwo.org/

Reports\%20\&\%20Publications/REDBOOK/kda_dwr.pdf). These data are published in annual reports (Kansas Water Office and Kansas Department of Agriculture, Division of Water Resources, 1993-2005). This data set has evolved as irrigation practices have changed. Center pivots with drop nozzle irrigation systems were incorporated into the data set beginning in 1992.

Information is reported by point of diversion and includes the amount of water pumped, acres irrigated, crop type, and irrigation system type. Follow-up letters and phone calls are made to obtain missing data and to confirm or correct anomalous data (for example, excessive application rates). Where water use from multiple points of diversion is reported with a single total for acres irrigated, the acres are prorated among the diversion points. Beginning with the 2001 irrigation data, there has been an increasing number of reports with missing data due to decreased follow-up efforts. The most common problems are missing acres, crop type, and system type.

Irrigation data used in this report were aggregated by county and by GMD. The water-use reports were coded both with county location and GMD number. If the location was outside of the GMD boundary, it still was included in its respective county. For each of the five GMDs and the 33 counties included in this study, water use and acres irrigated were summarized by major crop type and by system type. Acre-feet per acre application rates were calculated for irrigation water use and also for each major crop and system type within each county and GMD. A description of crop and system types is provided in the following paragraphs.

Water applied to acres of single crops was identified for five major crop types - alfalfa, corn, grain sorghum, soybeans, and wheat. Because it is common to irrigate multiple crop types with single points of diversion, the largest number of irrigated acres reported on the questionnaire was for other or multiple crops. By prorating the numbers of acres reported for specified combinations of major crop types, an estimate was made of total acres of the five major crop types irrigated. This prorating technique assigned acres to each specified crop type in equal percentages. For example, if multiple crops were listed and corn and wheat were specified, 50 percent of the acres were assigned to each crop. If three crops were specified, then each was assigned 33.3 percent of the acres.

The major categories of irrigation system type identified on the water-use reports were flood, drip, center pivot, center pivot with drop nozzles, sprinkler other than center pivot, and combinations. In 1991, no identification of center pivot systems with drop nozzles was made, and the sprinkler other than center pivot was used primarily for golf course irrigation. Since 1992, water use and acres irrigated have been reported in increasing numbers for center pivot systems with drop nozzles.

\section{Soil Permeability Data}

Soil permeability for each county in the study area was estimated from soil permeability data for Kansas
(U.S. Department of Agriculture, 1994) (fig. 2). Permeability estimates were made for three randomly selected basins within each county that was underlain by the High Plains aquifer. Subbasins were those available from the Kansas stream statistics Web page (http://ks.water.usgs.gov/Kansas/studies/strmstats/ $\mathrm{ks} /$ ?test=basin). These three estimates were averaged to provide an estimate of the mean soil permeability for the county (table 1).

\section{Data Sets}

For this report, irrigation information was compiled into two sets of data. The first data set included irrigation data from 1991 through 2003 for each county having at least one-third of its area within a GMD (tables 1 and 2 at the back of this report). There were 33 counties that fell into this category (fig. 1). Data included irrigation water use, total acres irrigated, and irrigation application rates for system type (flood, center pivot, center pivot with drop nozzle, and other systems) and for crop type (alfalfa, corn, grain sorghum, soybeans, wheat, and other/multiple use). In addition to this irrigation information, average annual precipitation and an estimate of mean county soil permeability were included (table 1). The second set of data (tables 3 and 4 at the back of this report) included all irrigation information for each GMD. Soil permeability for each GMD was not determined. The average precipitation for each GMD was estimated as the average precipitation for their respective meteorological region (fig. 1) and is included in table 5 at the back of this report.

\section{Effects of Irrigation Practices on Water Use}

\section{Statistical Analyses}

Kendall's Tau trend analysis (Kendall and Gibbons, 1990) was used in this report to determine objectively if trends in water-use data exist. Kendall's Tau is a mathematical technique that compares each data point in a time series with the other data points and determines the degree to which the data values are increasing or decreasing. The method provides a measure of the probability ( $p$ ) of the trend whether positive or negative. The user then can choose the degree of confidence of accepting the computed trend. In most cases, the user wants to be 95-percent confident that the trend is real. Therefore, $\mathrm{p}$ must be equal to or less than 0.05 . For a less confident 90 -percent level, p must be equal to or less than 0.10 . If $p=0.01$, the confidence would be 99 percent. The slope provided by Kendall's Tau analysis is a measure of the rate (magnitude) of the trend-the greater the slope, the greater the magnitude of the trend. A positive slope indicates a positive trend, and a negative slope indicates a negative trend. 


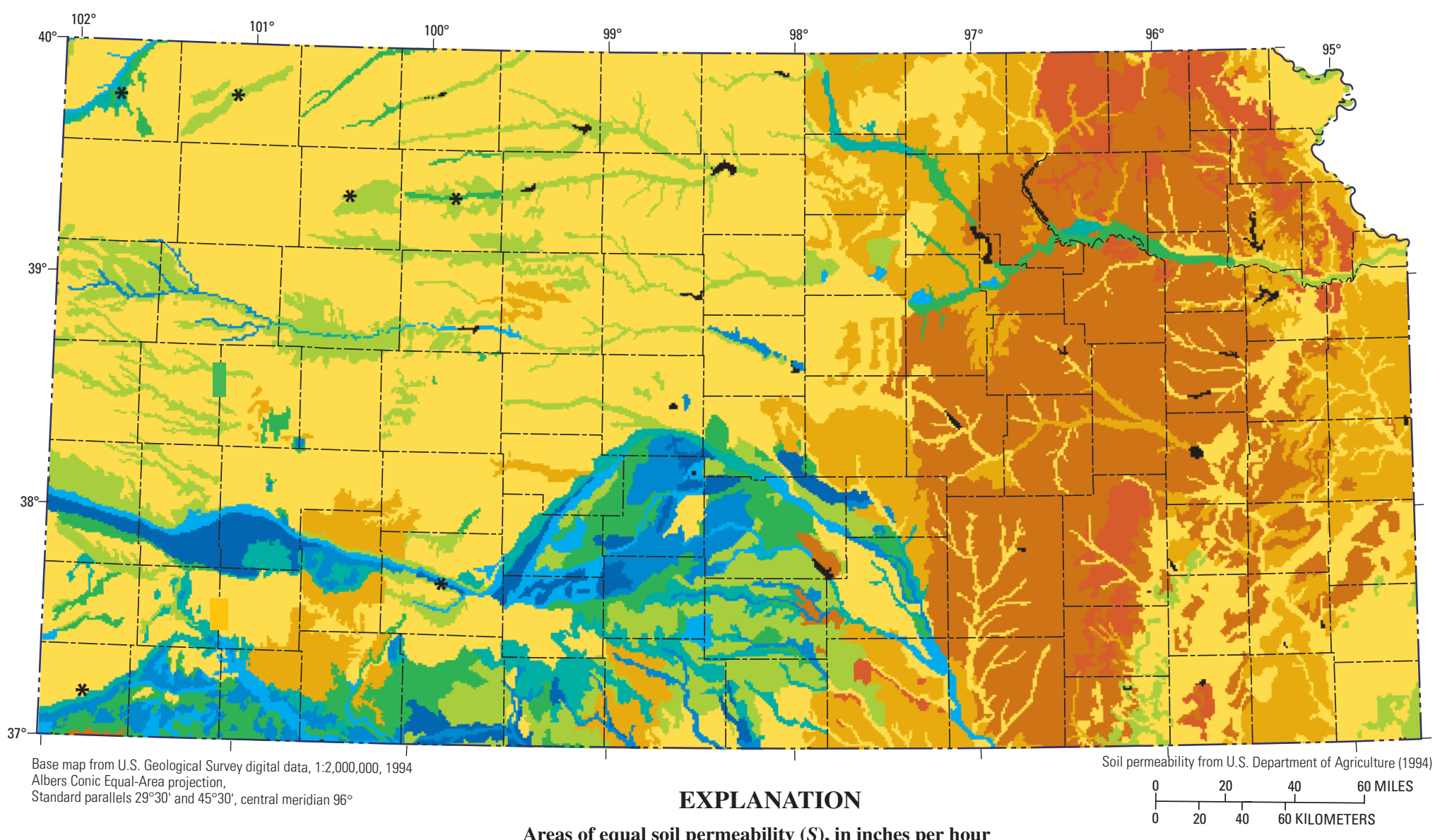

Areas of equal soil permeability $(S)$, in inches per hour

Figure 2. Soil permeability in Kansas.

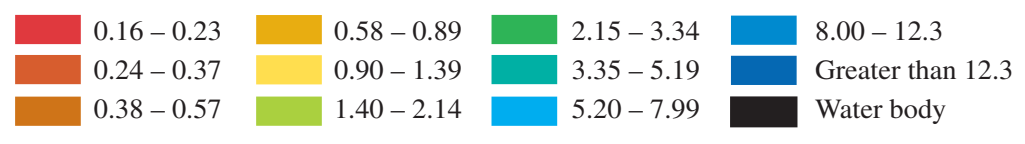




\section{Effect of Irrigation Practices on Water Use in the Groundwater Management Districts in the Kansas High Plains, 1991-2003}

Linear regression was used to determine the relation between an independent variable such as precipitation and a dependent variable such as water use. From this analysis a correlation coefficient was computed, which indicated the degree of fit between the two variables. The correlation coefficient, $R$, can vary from $R=0$, which is no fit, to $R=+1.0$, a perfect positive correlation, or to $\mathrm{R}=-1.0$, a perfect negative correlation. When the correlation coefficient is squared, the result provides a measure of the variability within the data. For example, a relation with $\mathrm{R}=0.8$, would have 64 percent of the variability within the relation explained by the independent variable.

\section{Precipitation Trends}

In analyzing irrigation data for trends, the factor that seems most important is the amount of precipitation. Irrigation supplies water needed for plant growth that is not supplied by rainfall, and the annual or growing-season precipitation should be linked directly to the amount of water applied through irrigation.

On average for the counties in the study area, 87 percent of the annual precipitation occurs from March through October. The remainder occurs during the winter months November through February. However, precipitation during the winter is crucial for antecedent moisture conditions for spring planting and winter wheat growth. Therefore, annual precipitation values were used in most of the analyses described in this report.

Figure 3 shows the distribution of annual precipitation for four of the meteorological regions $(1,4,7$, and 8) in Kansas. Table 6 (at the back of this report) is a compilation of Kendall's trend analysis (Kendall and Gibbons, 1990) of various time intervals of annual regional precipitation. The intervals were 1895 to 2003,1950 to 2003,1970 to 2003,1980 to 2003 , and finally the period 1991 through 2003, which is the time interval that includes the irrigation data described in this report. To be significant at the 95-percent confidence interval, the p-value must be equal to or less than 0.05 . For the first four periods, the only significant trend (positive) was during the period 1950 to 2003 in meteorological region 9 in southeastern Kansas (fig. 1). For 1991 through 2003, two regions had significant decreases in precipitation. Those decreases occurred in northwestern Kansas (region 1) and west-central Kansas (region 4). Average regional precipitation was defined as the average of the precipitation in meteorological regions $1,4,7$, and 8 . No statistically significant trend occurred in the average annual regional precipitation.

\section{Irrigation Trends by Groundwater Management Districts}

Total GMD irrigation water use for 1991 through 2003 was analyzed for trend, and it too did not show a significant trend. However, when average regional precipitation was compared with total GMD irrigation water use (fig. 4), a fairly good relation existed. The correlation coefficient was $\mathrm{R}=-0.77$, by which average annual regional precipitation explained 60 percent of the variability $\left(\mathrm{R}^{2}=0.60\right)$ of total GMD irrigation water use. The negative correlation indicates that more water was used during years with less precipitation.

A trend analysis for each of the GMDs was performed on precipitation, irrigation water use, acres irrigated, and irrigation application rate according to system type and crop type (tables 7-12). The rate of trend is reflected in the slope of the trend. The only significant trend (95-percent confidence level) in total irrigation water use was for GMD 2. The trend was positive and was slightly more than 6,000 acre-ft/yr (table 8). For total acres irrigated, all GMDs except GMD 1 had a statistically significant increase at the 95-percent confidence level (tables 8-11). However, the increase for GMD 3 (the largest) was only 99 acre/yr (table 9). GMD 5 had the largest significant increase in acres irrigated at 4,617 acre/yr (table 11), GMD 4 had an increase of 2,330 acre/yr, and GMD 2 had an increase of $2,843 \mathrm{acre} / \mathrm{yr}$. The trend for total acres irrigated for all GMDs was a positive $8,311 \mathrm{acre} / \mathrm{yr}$ and was significant at the 90-percent level ( $\mathrm{p}=0.059)$ (table 12). In every GMD the number of acres decreased significantly for flood and center pivot systems without drop nozzles and increased significantly for center pivot systems with drop nozzles (tables 7-12).

The total number of acre-feet used and number of acres irrigated, by system type and crop type, varied among GMDs because of their comparative sizes and geographic locations. Figure $5 A-F$ shows the irrigation water use in thousands of acre-feet, figure $6 A-F$ shows the number of acres irrigated for each system type, and figure $7 A-F$ shows the number of acres irrigated for each crop type. GMD 3 had the largest amount of irrigation water use and the most acres irrigated of any of the GMDs-63 percent of the total GMD water use and 57 percent of the total acres. For the number of acres irrigated by system type, the graphs in figure 6 show the decrease in acres irrigated using flood and center pivot systems and the concurrent increase in acres irrigated by center pivot systems with drop nozzles. This trend is apparent in each GMD (tables 7-12). GMD 3 had the largest number of acres converted to drop nozzle irrigation, approximately 800,000 . The number of acres planted to corn (after other or multiple crops were prorated) was the largest for any crops for all GMDs (fig. 7).

Trends in irrigation water use and number of acres irrigated for each crop suggested that more water-intensive crops are being irrigated simultaneously with the trend toward more acres irrigated by center pivots with drop nozzles. During 19912003 , irrigation water use and acres irrigated increased significantly (at the 95-percent confidence level) for alfalfa, corn, and soybeans (table 12). Concurrently, the less water-intensive crops of grain sorghum and wheat had significant (at the 95percent confidence level) negative trends in number of acres irrigated. A conclusion that can be drawn from these analyses is that, although total irrigation water use for all GMDs showed no statistically significant trend, an apparent increased efficiency 
A. Region 1, Groundwater Management District 4 (northwest Kansas, fig. 1)

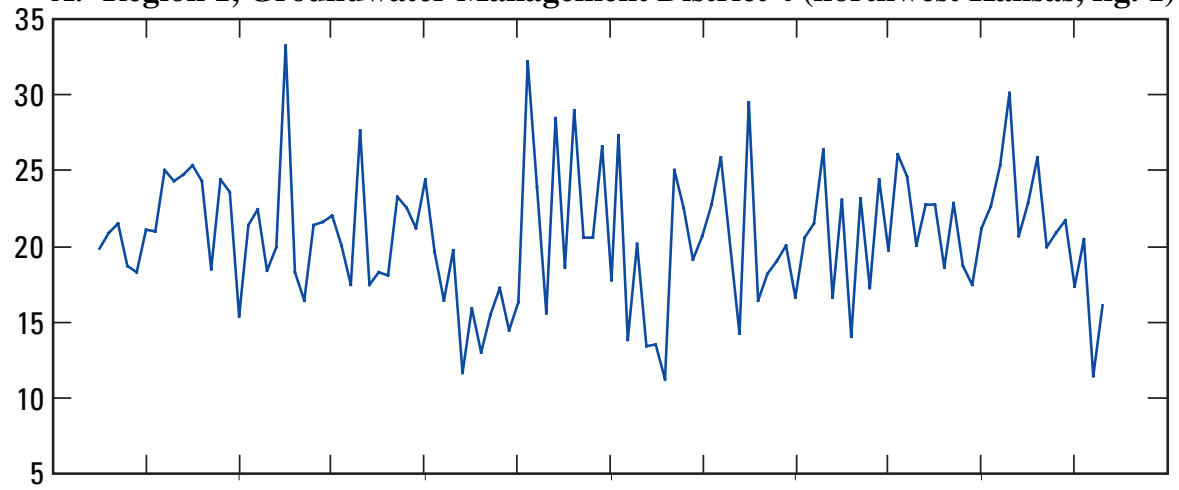

B. Region 4, Groundwater Management District 1 (west-central Kansas, fig. 1)

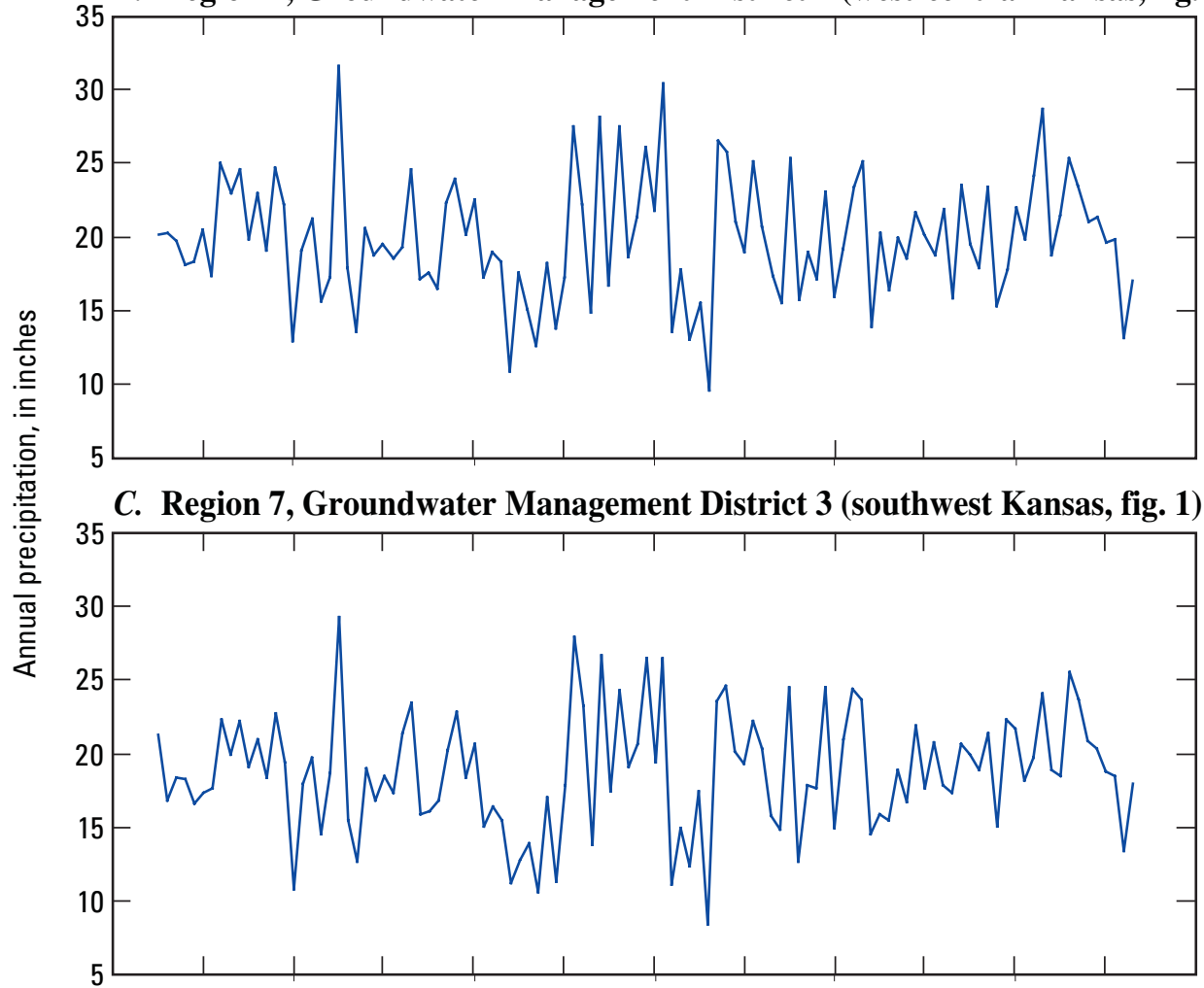

D. Region 8, Groundwater Management Districts 2 and 5 (south-central Kansas, fig. 1)

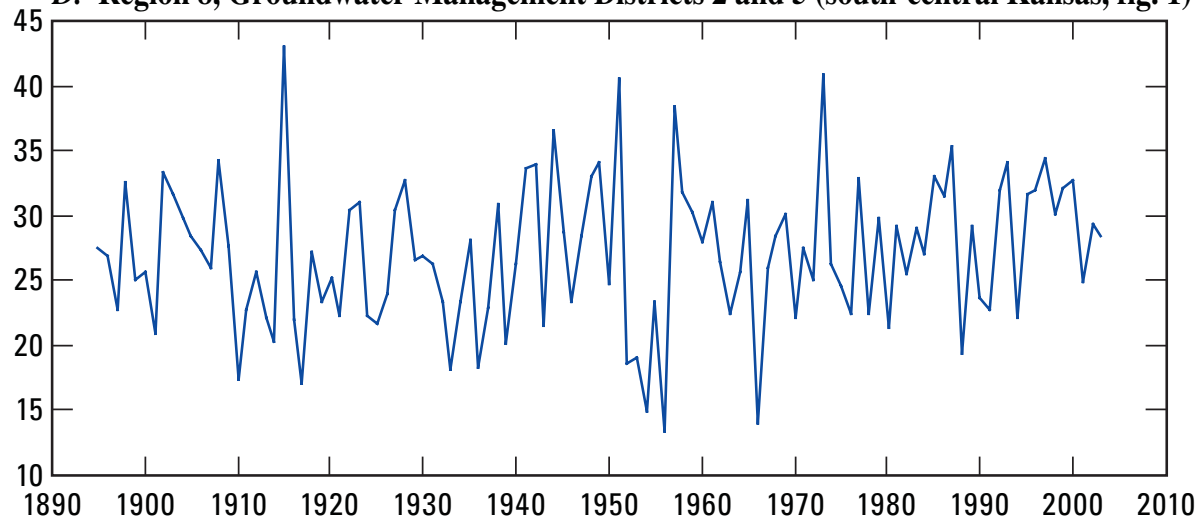

Figure 3. Annual precipitation by meteorological region, 1895-2003 (data from National Oceanic and Atmospheric Administration, 1895-2005). 


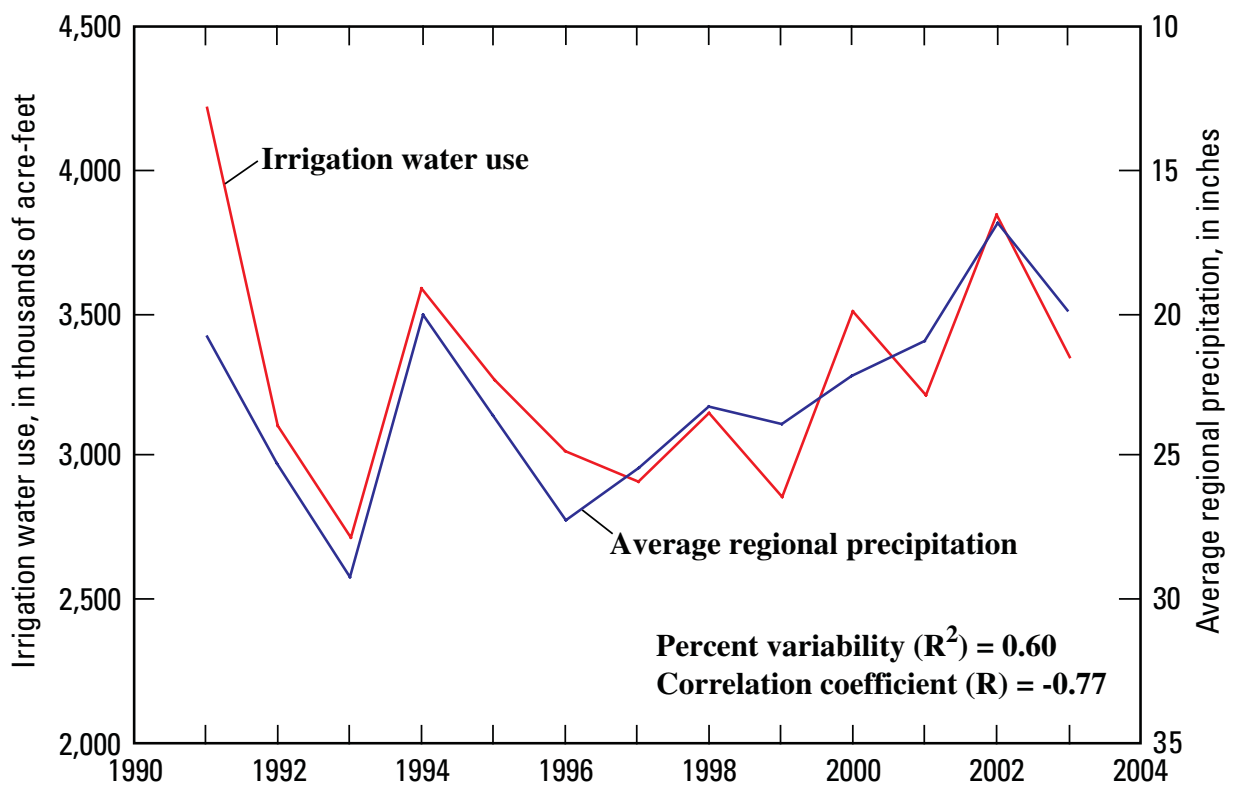

Figure 4. Relation between irrigation water use for Groundwater Management Districts and average precipitation for meteorological regions 1, 4, 7, 8, 1991-2003 (water-use data from Kansas Water Office and Kansas Department of Agriculture, Division of Water Resources, 1993-2005, and precipitation data from National Oceanic and Atmospheric and Administration, 1895-2005).

of center pivot irrigation systems with drop nozzles has allowed more water-intensive crops to be grown on more irrigated acres.

\section{Irrigation Trends by County}

Kendall's Tau analyses were performed on county data for irrigation water use and total acres irrigated. Trend analyses also were performed on annual county precipitation for comparison. These results are listed in table 1 . The largest significant positive trends at the 95-percent confidence level for either irrigation water use or total acres irrigated occurred in Harvey, Pratt, Reno, Seward, Sherman, and Stevens Counties. The largest significant negative trends at the 95-percent confidence level for either irrigation water use or total acres occurred in Grant, Haskell, and Stanton Counties.

\section{Effect of Precipitation on Irrigation Water Use}

As noted in the section on "Precipitation Trends," irrigation water use has a general link to precipitation. To test this relation in more detail, average annual county precipitation was compared to irrigation water use for each county for each year from 1991 through 2003. Individual county analyses showed a range of correlation coefficients from a high of $\mathrm{R}=0.91$ for Pawnee County to a low of $\mathrm{R}=0.07$ for Reno County (table 13 ). The average correlation coefficient for all 33 counties was $\mathrm{R}=0.58$.
Irrigation application rate did not correlate with precipitation amounts (average correlation coefficient of 0.12).

When irrigation water use in each GMD was considered with its respective regional precipitation, GMD 4 had the best relation, with 68 percent of the variability $\left(R^{2}=0.68\right)$ explained using annual precipitation from region 1 precipitation (table 14). Sixty percent of the irrigation water use for all of the GMDs was explained by the average regional precipitation (average of regions 1, 4, 7, and 8). When this relation was examined on a year-to-year basis (fig. 8), the data generally fell into a pattern of 1991 through 1996 data (center pivots with drop nozzles less than 10 percent of total acres irrigated, from table 3) plotting above the line of regression, while data from 1997 through 2003, except 2000 (center pivots with drop nozzles greater than 28 to 60 percent of total acres irrigated), plotting below the line of regression. If the data are separated into two data sets, the resulting regression lines show a decrease in irrigation water use with respect to precipitation from approximately 400,000 acre-ft at 20 in. of precipitation to 300,000 acre$\mathrm{ft}$ at $25 \mathrm{in}$. of precipitation for all GMDs. The difference between the $y$-intercepts for the two regression lines was found to be statistically significant using the statistical t test.

Because the shortage of precipitation necessitates the application of irrigation water, two equations were developed to factor in the effect of precipitation variability on collective irrigation water use for all GMDs. These equations were generated to explore the effect of annual precipitation on the amount of irrigation water use. A regression equation was developed to estimate adjusted water use from the relation between average 
A. Groundwater Management District 1 (west-central Kansas, fig. 1)
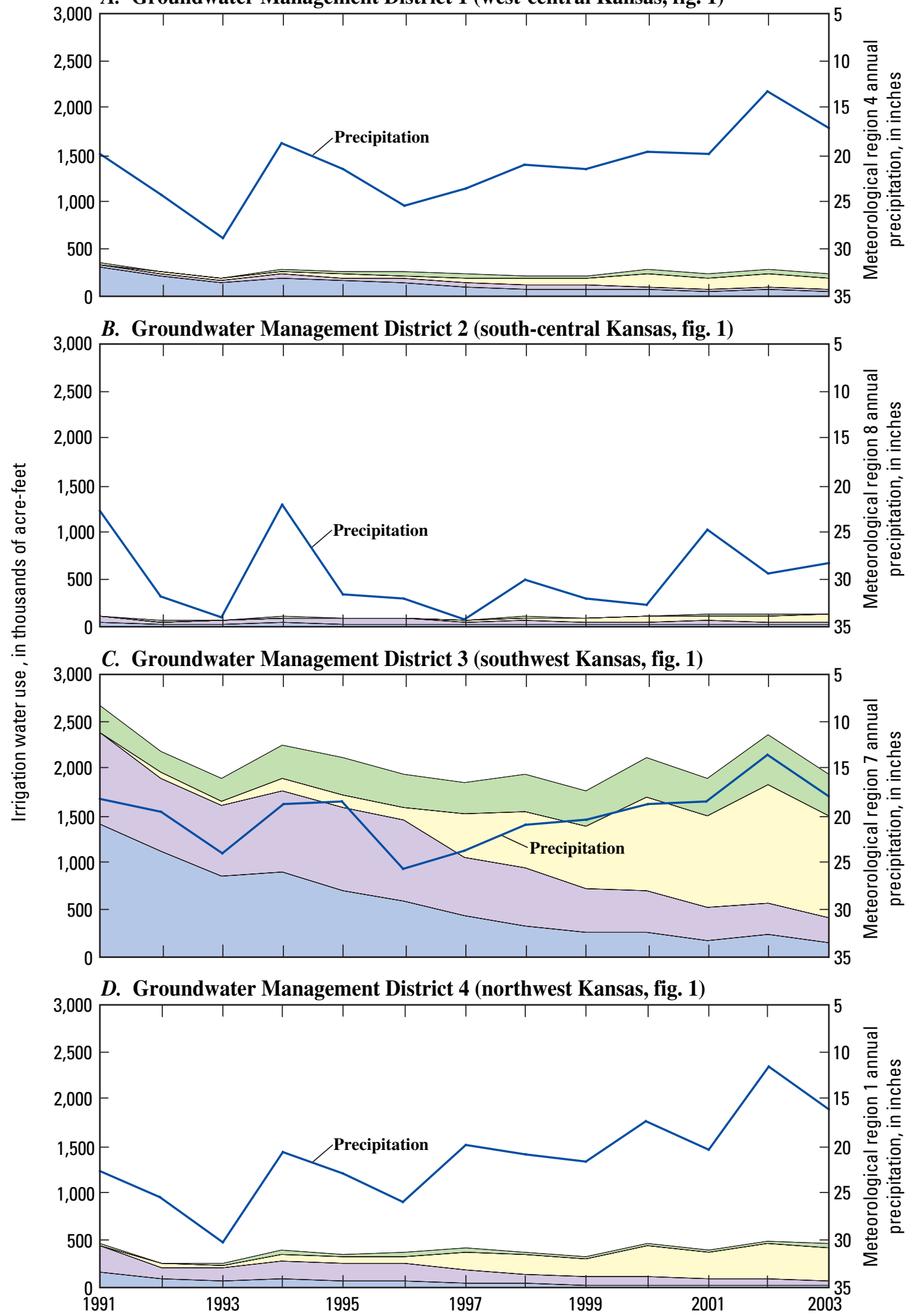

Figure 5. Irrigation water use and annual regional precipitation by Groundwater Management District, 19912003 (water-use data from Kansas Water Office and Kansas Department of Agriculture, Division of Water Resources, 1993-2005, and precipitation data from National Oceanic and Atmospheric Administration, 1895-2005). 


\section{E. Groundwater Management District 5 (south-central Kansas, fig. 1)}

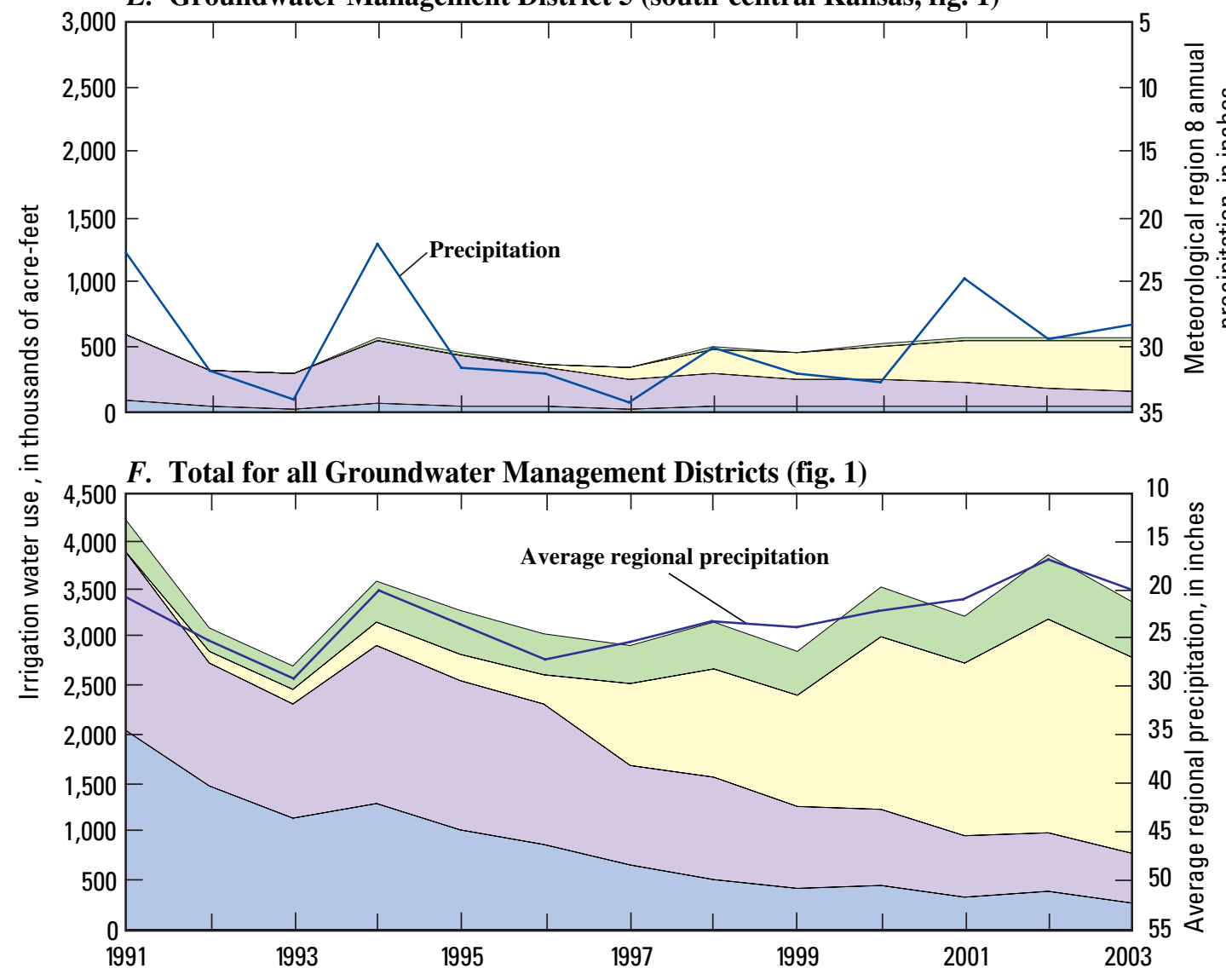

EXPLANATION

Type of irrigation system

Figure 5. Irrigation water use and annual regional precipitation by Groundwater Management District, 19912003 (water-use data from Kansas Water Office and Kansas Department of Agriculture, Division of Water Resources, 1993-2005, and precipitation data from National Oceanic and Atmospheric Administration, 18952005).-Continued

annual precipitation for the four meteorological regions in the study area and the measured irrigation water use. The equation becomes:

Estimated water use (acre-feet) $=96,300 x$

(average regional precipitation, inches) $+5,500,000$.

The second equation used to adjust irrigation water use for the variability of precipitation consisted of multiplying each annual water use times the ratio of the 13-year precipitation average to the annual precipitation:

Estimated water use $=$ Annual water use $\mathrm{x}$ (Average regional precipitation) Annual regional precipitation
As noted in a preceding section, the measured irrigation water use had no statistically significant trend during the 13-year study period. The regression-equation estimate (equation 1) showed a positive trend in water used that had a p-value of 0.076 (that is, significant at the 90-percent level). The estimate of water use using the ratio of average to annual regional precipitation (equation 2) resulted in a statistically significant (at the 95-percent confidence level) negative trend in water use (fig. 9). The trend analyses of the results from the comparison of two methods of adjusting irrigation water use for precipitation were contradictory. This demonstrates the problem of adjusting irrigation water use for precipitation where both equations are valid.

The average total water received for each year for all the irrigated area within the GMDs was estimated from the average 
A. Groundwater Management District 1 (west-central Kansas, fig. 1)

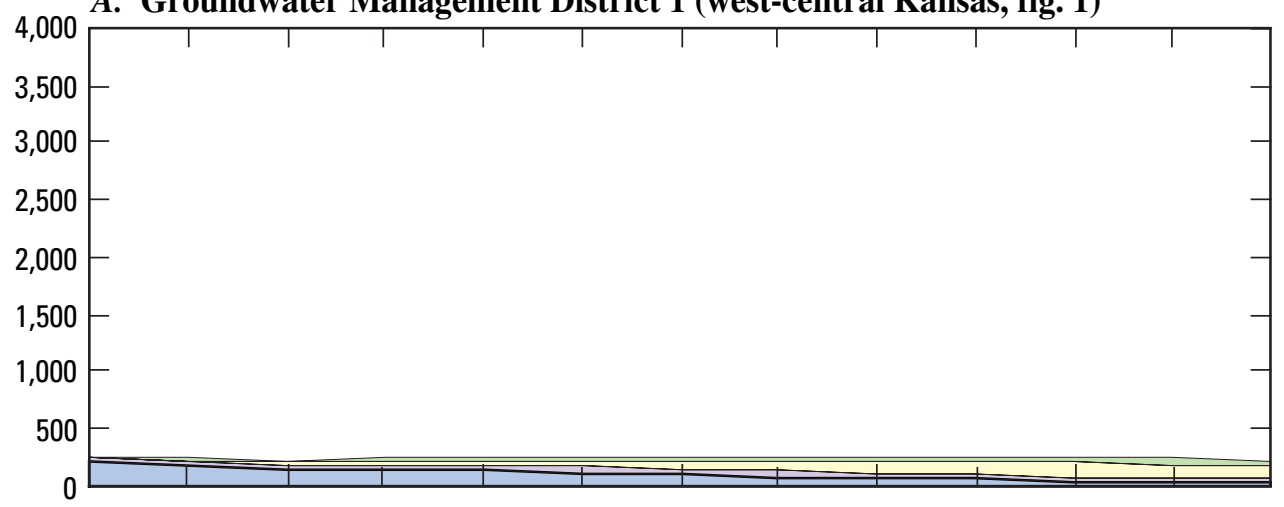

B. Groundwater Management District 2 (south-central Kansas, fig. 1)

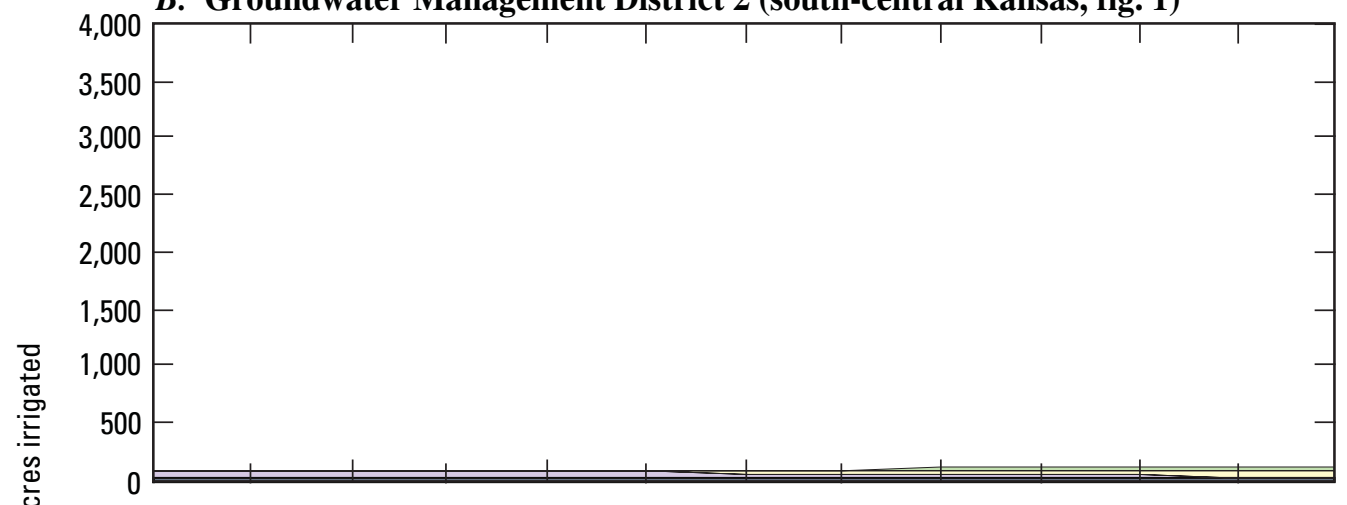

C. Groundwater Management District 3 (southwest Kansas, fig. 1)

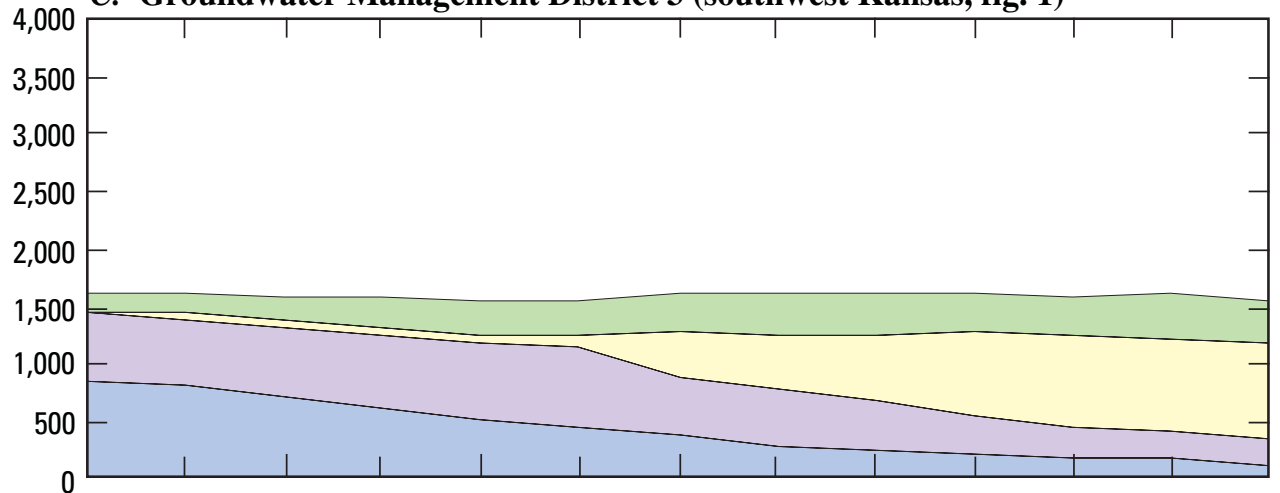

D. Groundwater Management District 4 (northwest Kansas, fig. 1)

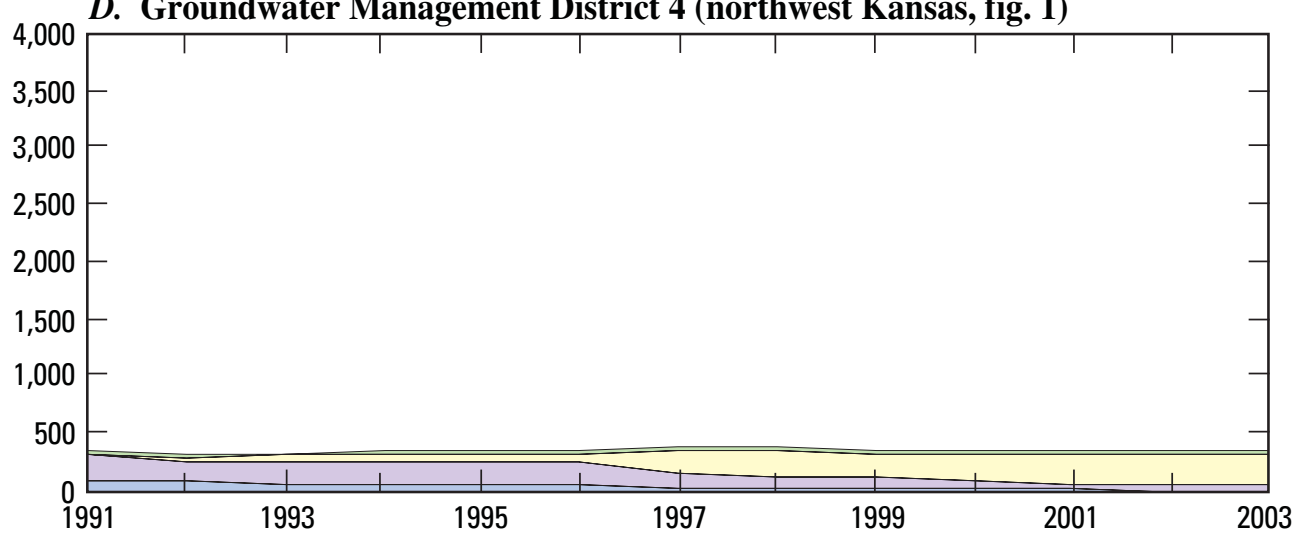

Figure 6. Acres irrigated by system type for Groundwater Management Districts, 1991-2003 (data from Kansas Water Office and Kansas Department of Agriculture, Division of Water Resources, 1993-2005). 


\section{E. Groundwater Management District 5 (south-central Kansas, fig. 1)}

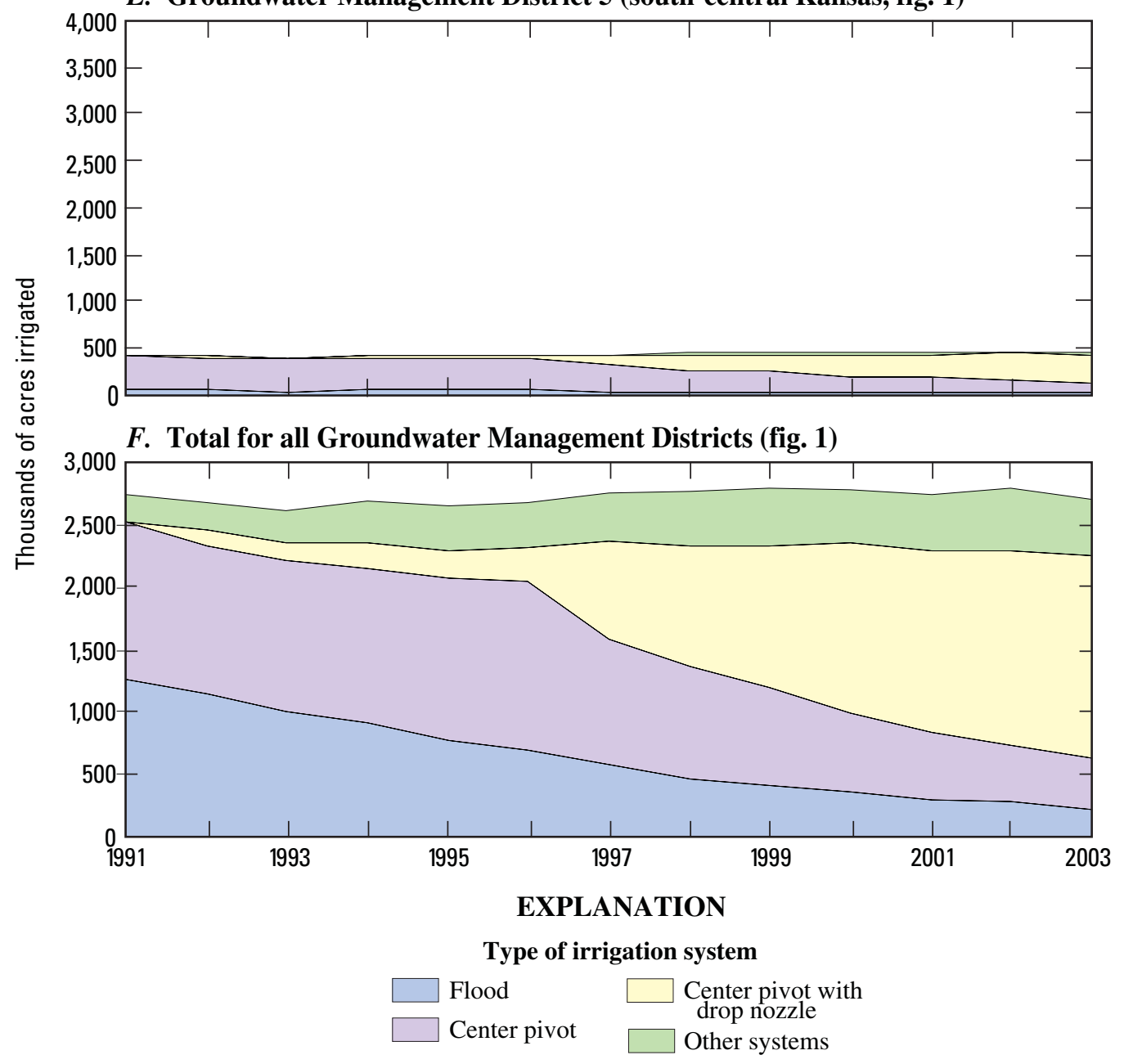

Figure 6. Acres irrigated by system type for Groundwater Management Districts, 1991-2003 (data from Kansas Water Office and Kansas Department of Agriculture, Division of Water Resources, 19932005).-Continued

precipitation over the study area plus the average irrigation application rate. Irrigation water use in acre-feet was divided by the number of acres irrigated for each year to obtain the average irrigation application rate. This number was converted to inches and added to the average precipitation for the study area. The results are listed in table 16 and shown in figure 10. The graph shows that the total water applied has decreased from 1991 to 2003. Kendall's Tau analysis on this time series shows a statistically significant decrease $(\mathrm{p}=0.003)$ with a Kendall's slope of $-0.46 \mathrm{in} / \mathrm{yr}$. Linear regression on this data results in a slope of $-0.48 \mathrm{in} / \mathrm{yr}$ and an $\mathrm{R}^{2}=0.60$.

\section{Effect of Drop Nozles on Irrigation Water Use}

One method of analyzing the effect of drop nozzle irrigation systems on irrigation water use is to compare irrigation application rates for each year. From the information in table 3 , graphs comparing flood and center pivot application rates to center pivots with drop nozzle application rates were constructed for each GMD and for the combination of all GMDs. A least-squares regression was performed on each data set, and the resulting relation was forced to a y-intercept of 0 . The slope of the resulting line shows the percentage of water used by one irrigation system compared to the other. For the centerpivot to drop-nozzle comparison, slopes ranged from 0.98 to 1.05 (fig. $11 A-E$ ) for individual GMDs. When irrigation water use and total acres irrigated for each year were used to compute a total application rate, the slope was 0.98 (fig. $11 F$ ), indicating that the drop nozzle application rate used approximately 2 percent less water than the center pivot application rate. When flood irrigation was compared with the drop nozzle application 
A. Groundwater Management District 1 (west-central Kansas, fig. 1)

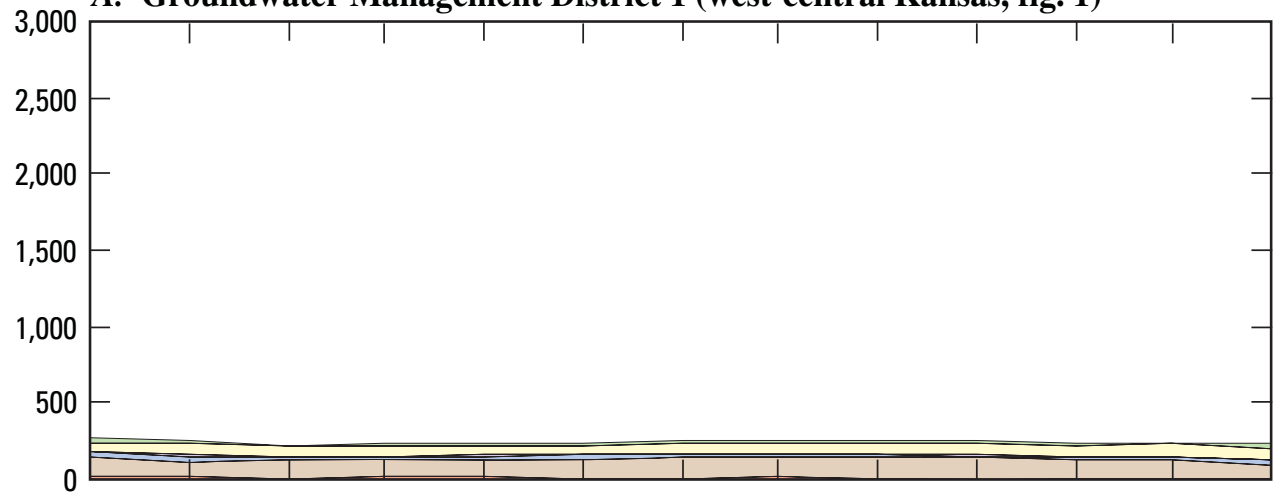

B. Groundwater Management District 2 (south-central Kansas, fig. 1)

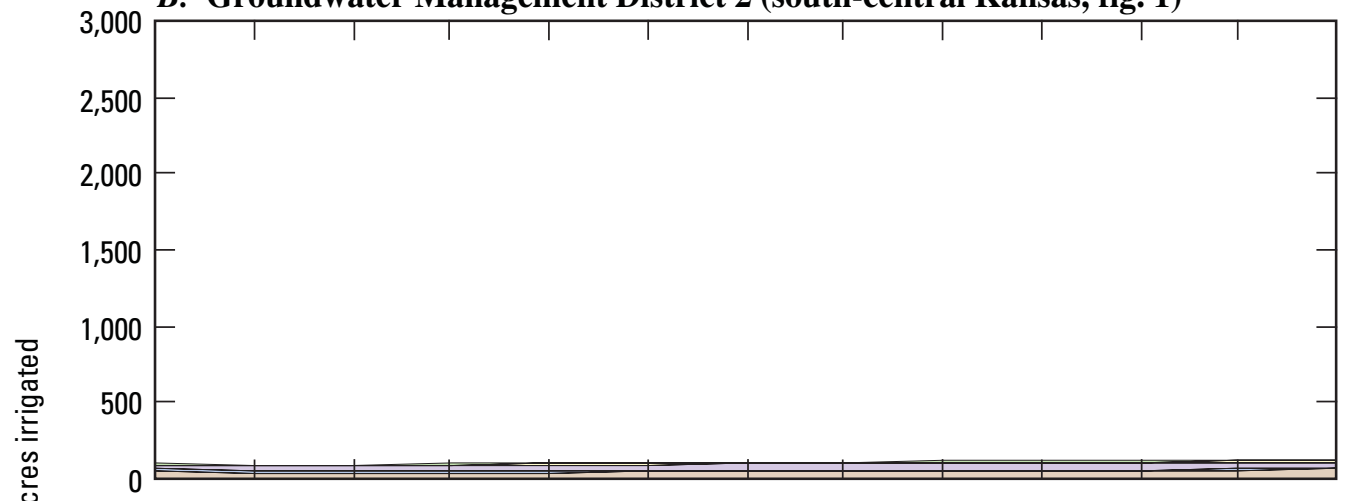

C. Groundwater Management District 3 (southwest Kansas, fig. 1)
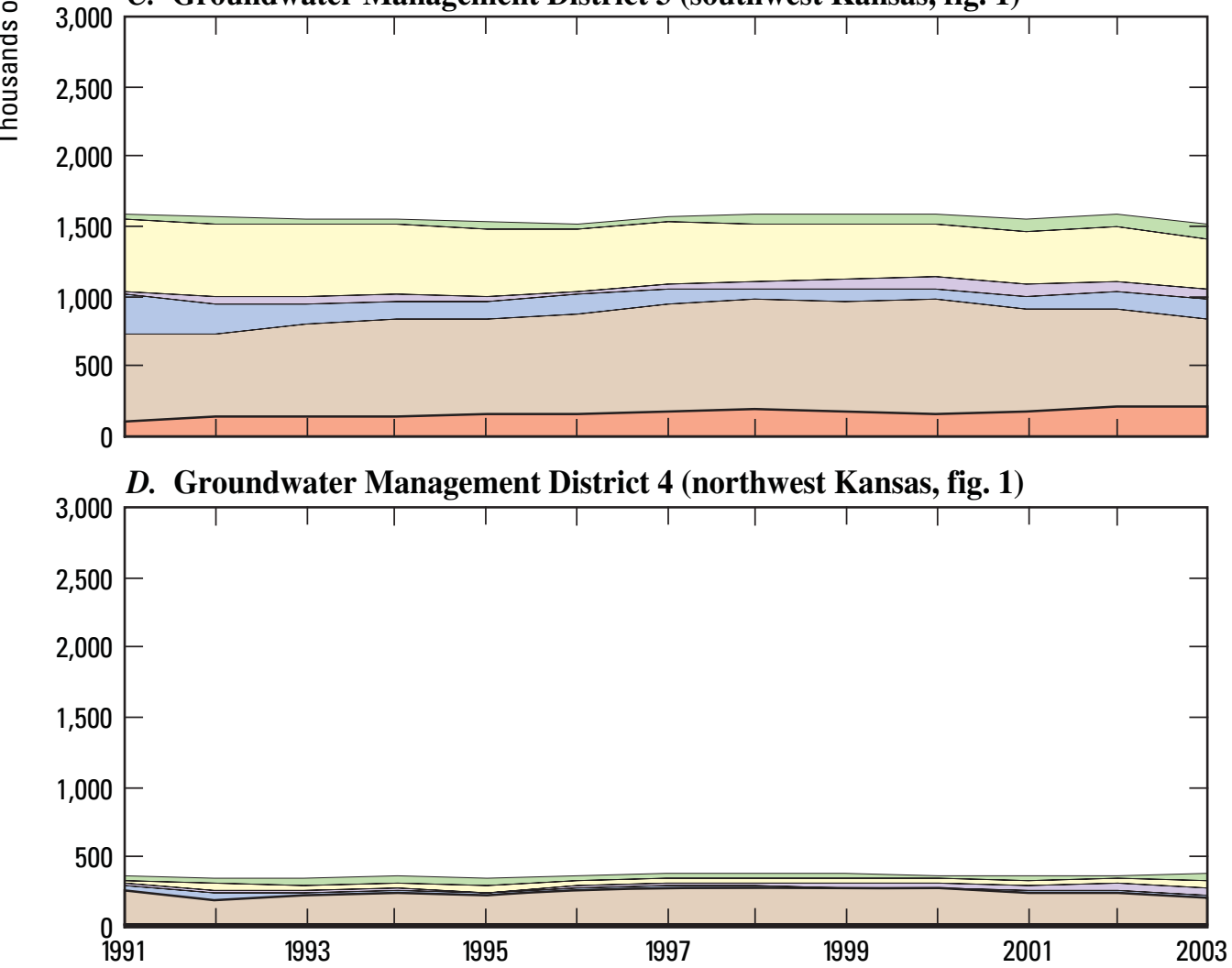

Figure 7. Acres irrigated by crop type for Groundwater Management Districts, 1991-2003 (data from Kansas Water Office and Kansas Department of Agriculture, Division of Water Resources, 1993-2005). 


\section{E. Groundwater Management District 5 (south-central Kansas, fig. 1)}

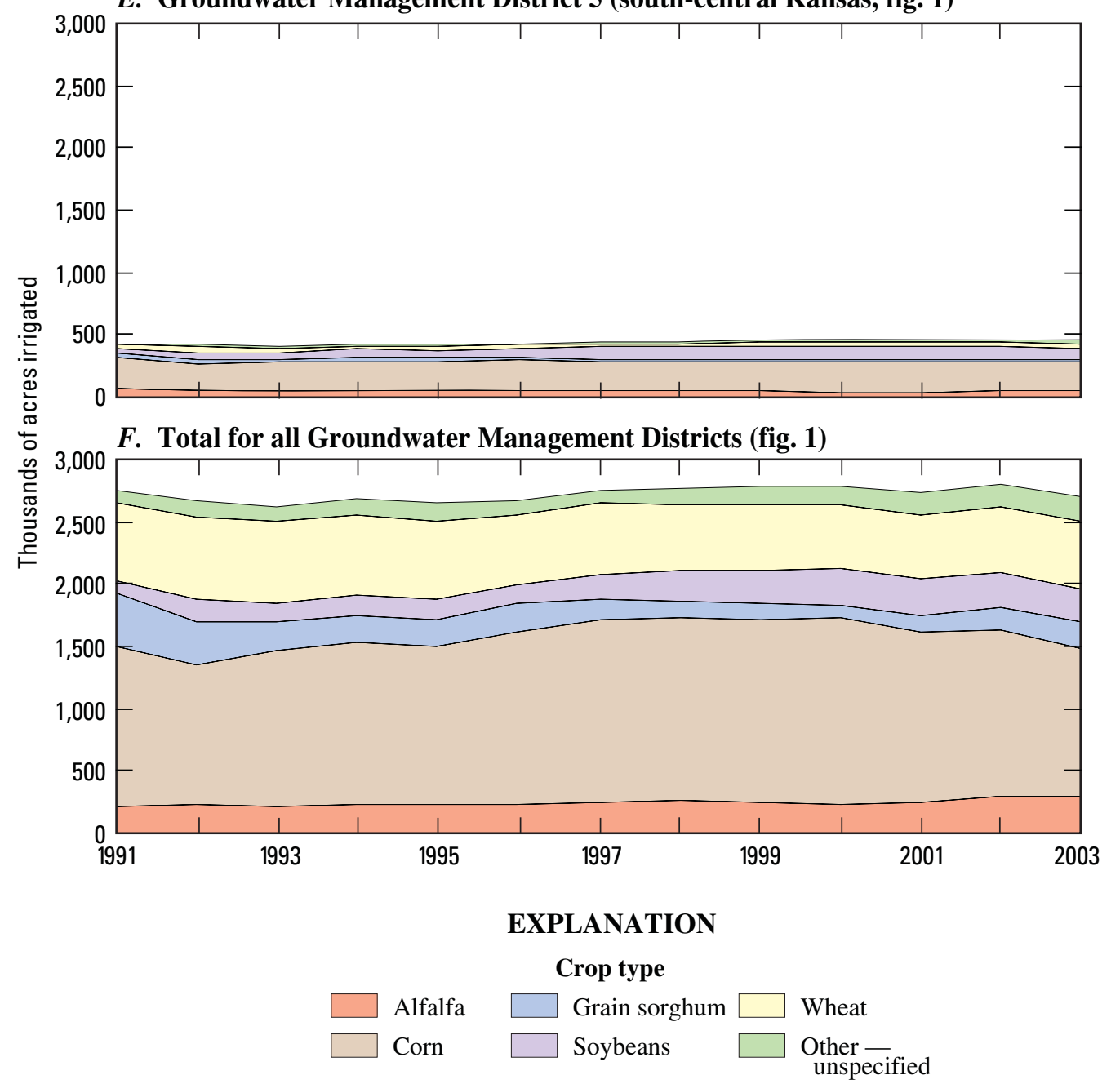

Figure 7. Acres irrigated by crop type for Groundwater Management Districts, 1991-2003 (data from Kansas Water Office and Kansas Department of Agriculture, Division of Water Resources, 19932005).-Continued

rate, the slope ranged from 0.82 to 1.10 (fig. $12 A-F$ ), and for the total application rate for all GMDs, the slope was 0.92

(fig. $12 F$ ). This analysis indicated that drop nozzle systems on average used 8 percent less water than flood irrigation systems. An analysis was done using the county data as well. Both GMD and county analyses are summarized in table 17 for the time period 1992-2003.

\section{Effect of Crop Conversion on Irrigation Water Use}

Total irrigated acres increased from 2,681,000 to 2,746,000 acres between 1991-93 and 2001-03, a 2.4-percent increase; however, during this time, the total acres of corn, alfalfa, and soybeans increased from 25 to 40 percent, depending upon whether total prorated acres $(1,483,000$ to $1,859,000$ acres) or only the actual corn-alfalfa-soybean reported acres (828,000 to 1,167,000 acres) are used (table 12). Using either figure, there has been a substantial increase in crops that traditionally are water intensive. When the average irrigation water use for the first 3 years (1991-93, $3,344,000$ acre-ft) is compared with the last 3 years (2001-03, 3,470,000 acre-ft) (table 3), there has been a 3.8-percent increase in irrigation water use from one period to the next. This increase in the total number of acres and the number of acres with water-intensive crops probably would result in an increase in water use in excess of the actual increase were irrigation efficiency not increasing.

\section{Correlation of Factors Affecting Irrigation Water Use}

An attempt was made to correlate the factors that affect irrigation water use using data from tables 1 and 2. Table 18 is a correlation matrix from a stepwise multiple regression analysis for water-use factors for the 33-county data set. Correlation 


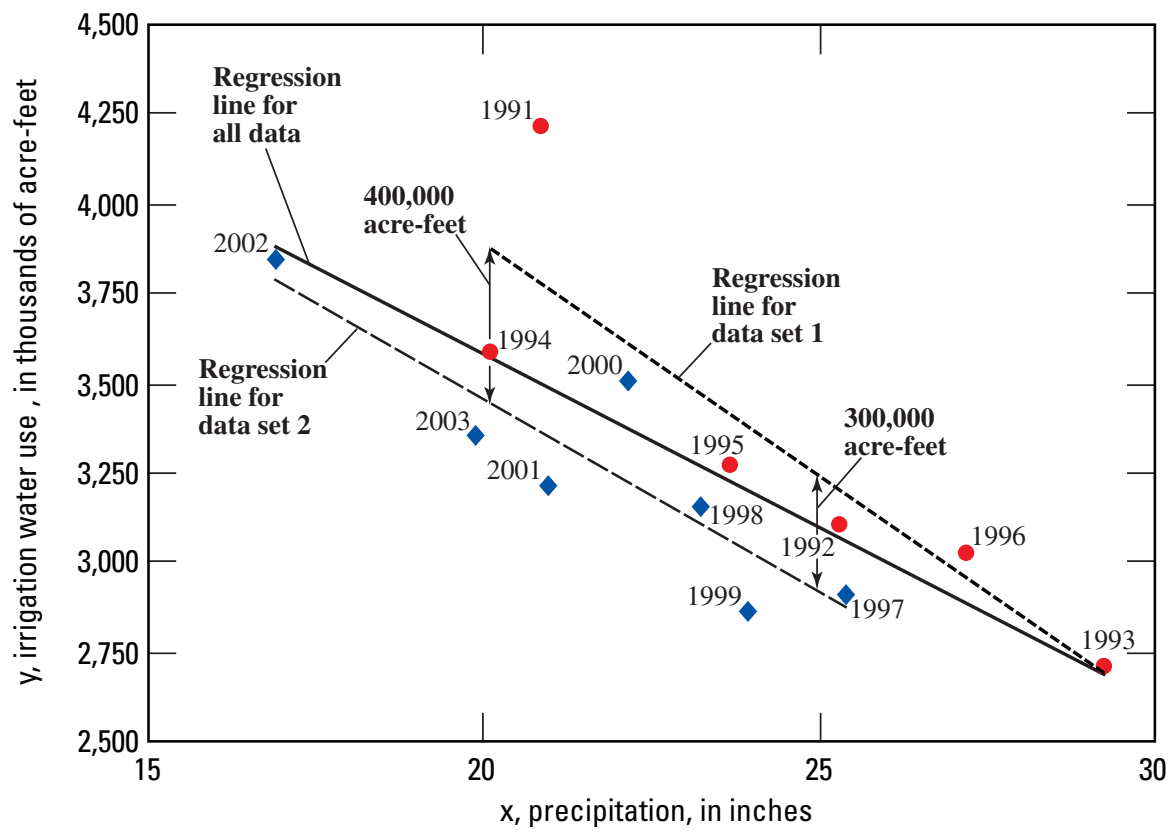

EXPLANATION

- Data set 1 (1991-96)

- Data set 2 (1997-2003)

$\begin{array}{lll}\text { All data } & \mathbf{y}=-\mathbf{9 6 , 3 0 0 x}+5,500,000 & \mathbf{R}^{2}=\mathbf{0 . 6 0} \\ \text { Data set 1 } & \mathbf{y}=-\mathbf{1 2 9 , 0 0 0 x}+\mathbf{6 , 4 7 0 , 0 0 0} & \mathbf{R}^{2}=\mathbf{0 . 7 7} \\ \text { Data set 2 } & \mathbf{y}=-107,000 x+5,600,000 & \mathbf{R}^{2}=\mathbf{0 . 7 9}\end{array}$

Figure 8. Relation between irrigation water use and precipitation, 1991-2003.

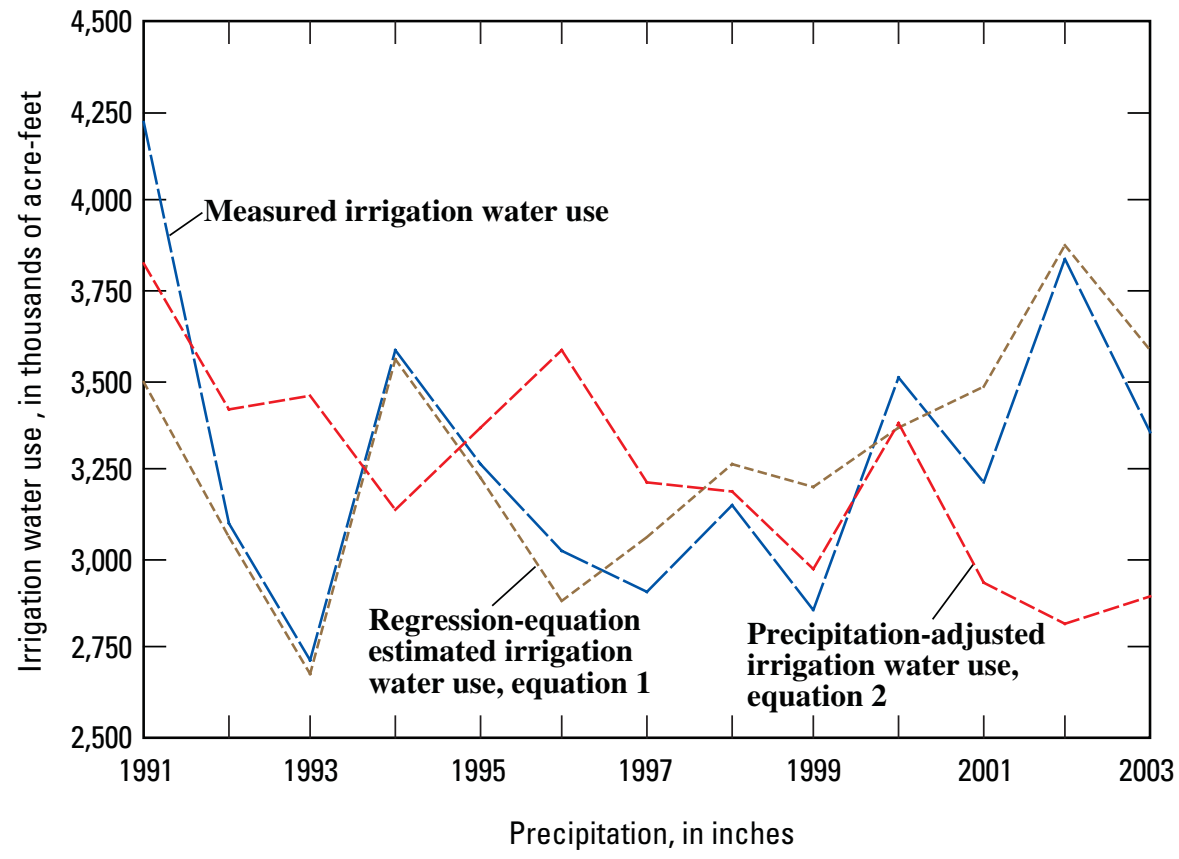

Figure 9. Comparison of measured irrigation water use and precipitation-adjusted irrigation water use for Groundwater Management Districts using two different equations for adjustment. 


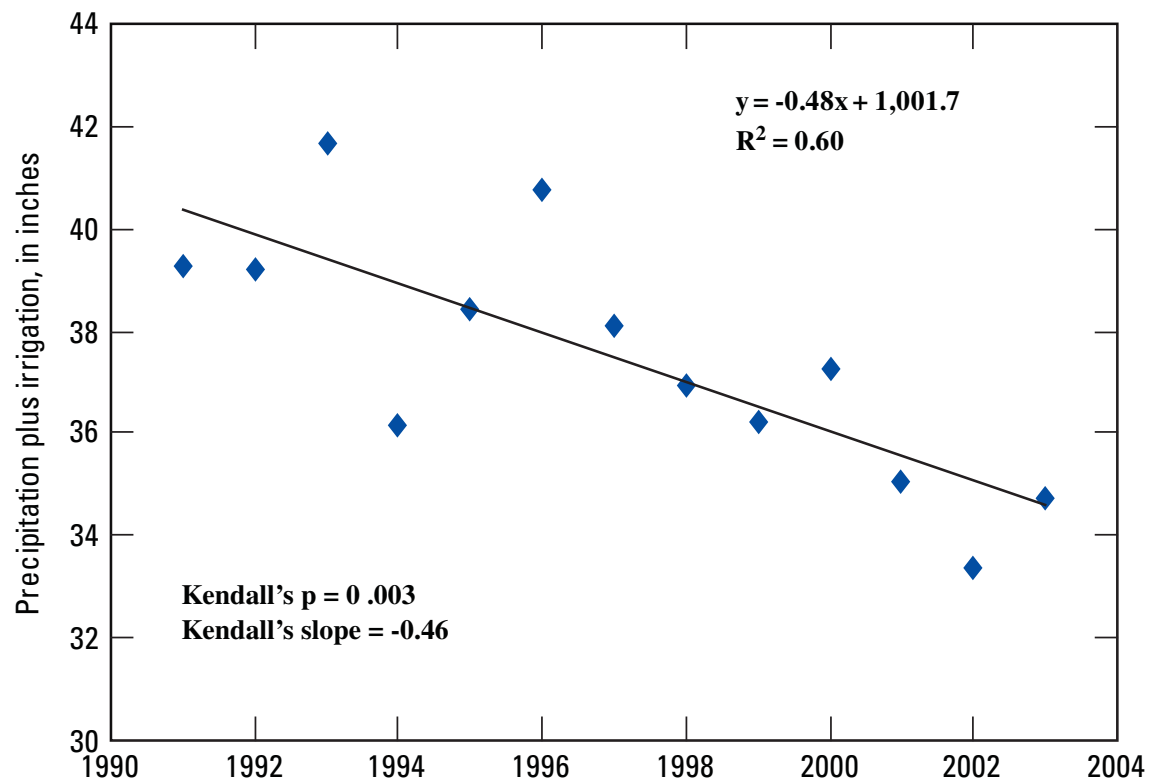

Figure 10. Average precipitation plus irrigation-applied water for all Groundwater Management Districts, 1991-2003.

coefficients ranged from $\mathrm{R}=0.97$ to $\mathrm{R}=-0.67$. The major factor in irrigation water use is the total number of acres irrigated $(\mathrm{R}=0.97)$. The next most significant factor was average annual county precipitation or March-October precipitation. The best estimate of irrigation water use involved the use of total number of acres irrigated and the average annual regional precipitation. Table 19 summarizes the multiple regression analyses for irrigation water use and for irrigation application rates for corn and alfalfa, the two most common crops grown in the Kansas High Plains GMDs.

\section{Summary}

Data compiled for the High Plains region of Kansas that includes five Groundwater Management Districts (GMDs) were analyzed for trends in precipitation, irrigation system types, and crop types to determine the effects of irrigation practices on water use. For the study period 1991 through 2003, precipitation decrease significantly at the 95-percent confidence level in northwestern and west-central Kansas, but there was no statistically significant increase or decrease in southwestern and south-central Kansas. When the precipitation from four meteorological regions was averaged, there was no statistically significant trend (at the 95-percent confidence level) in precipitation. There was a good $(\mathrm{R}=-0.77)$ relation between average regional precipitation and irrigation water use for all GMDs. Actual irri- gation water use had no significant trend, but when adjusted for the ratio of the 1991-2003 average precipitation to yearly precipitation, there was a significant decrease in adjusted water use at the 95-percent confidence level. GMD 3 in southwestern Kansas used 63 percent of the total number of acre-feet of irrigation water and had 57 percent of the total acres irrigated within all of the GMDs.

The number of flood irrigation and center pivot irrigated acres decreased significantly during the study period. At the same time, the number of drop nozzle irrigated acres increased significantly. Irrigated acres of water-intensive corn, alfalfa, and soybeans also increased significantly, whereas irrigated acres of less water-intensive grain sorghum and wheat, and multiple crop type decreased significantly. Irrigation application rates for drop-nozzle irrigation systems averaged approximately 2 percent less than application rates for center pivot systems in a year-by-year comparison and were 8 to 11 percent less than application rates for flood irrigation systems. However, both were not statistically different from zero change.

The best estimator of irrigation water use incorporated total acres irrigated and average annual or March-October regional precipitation. A conclusion that can be drawn from these analyses is that, although irrigation water use for all GMDs showed no statistically significant trend, an apparent increased efficiency of center pivots irrigation systems with drop nozzles has allowed more water-intensive crops to be grown on more irrigated acres. 
A. Groundwater Management District 1 (west-central Kansas, fig. 1)
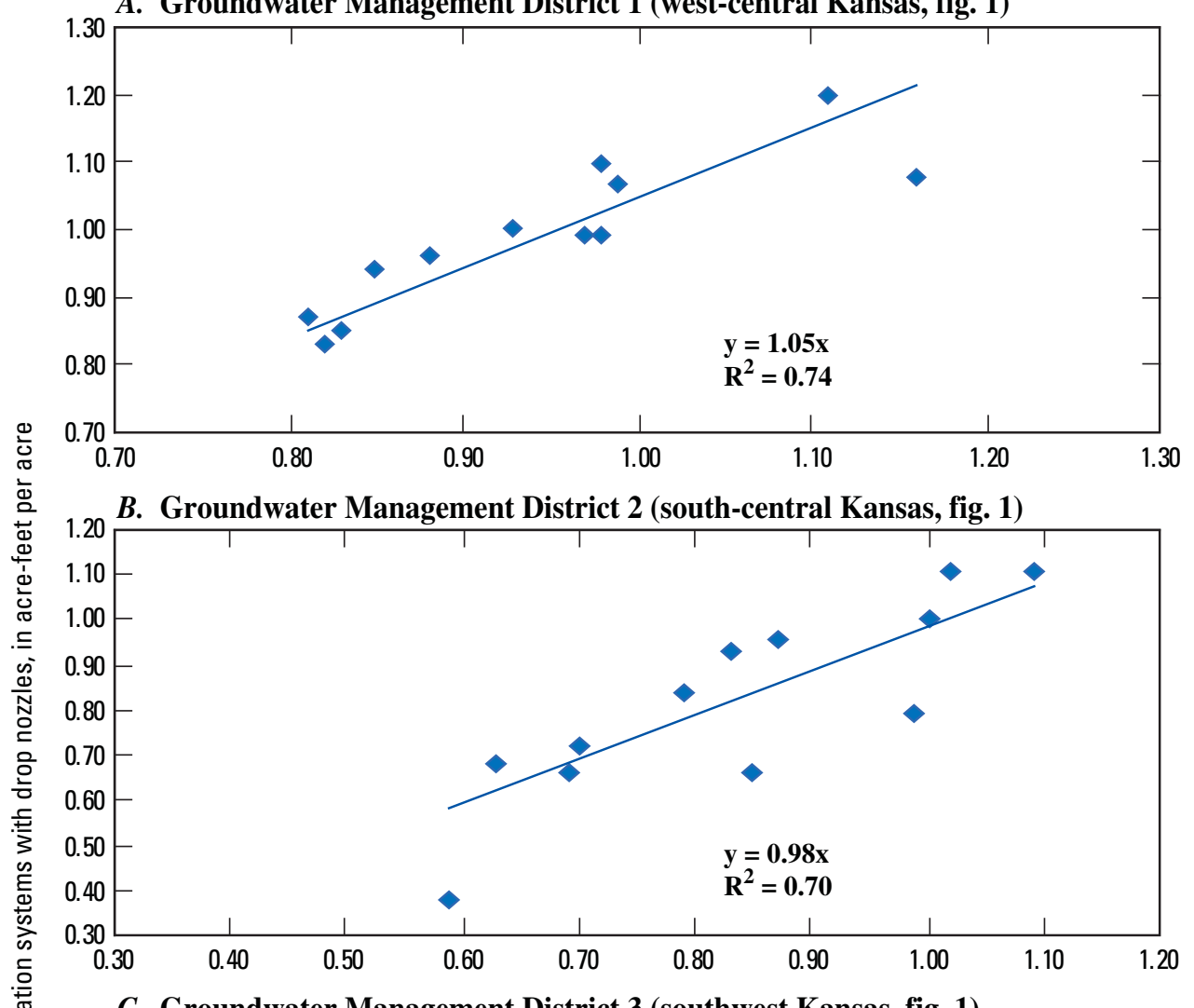

1.60 C. Groundwater Management District 3 (southwest Kansas, fig. 1)

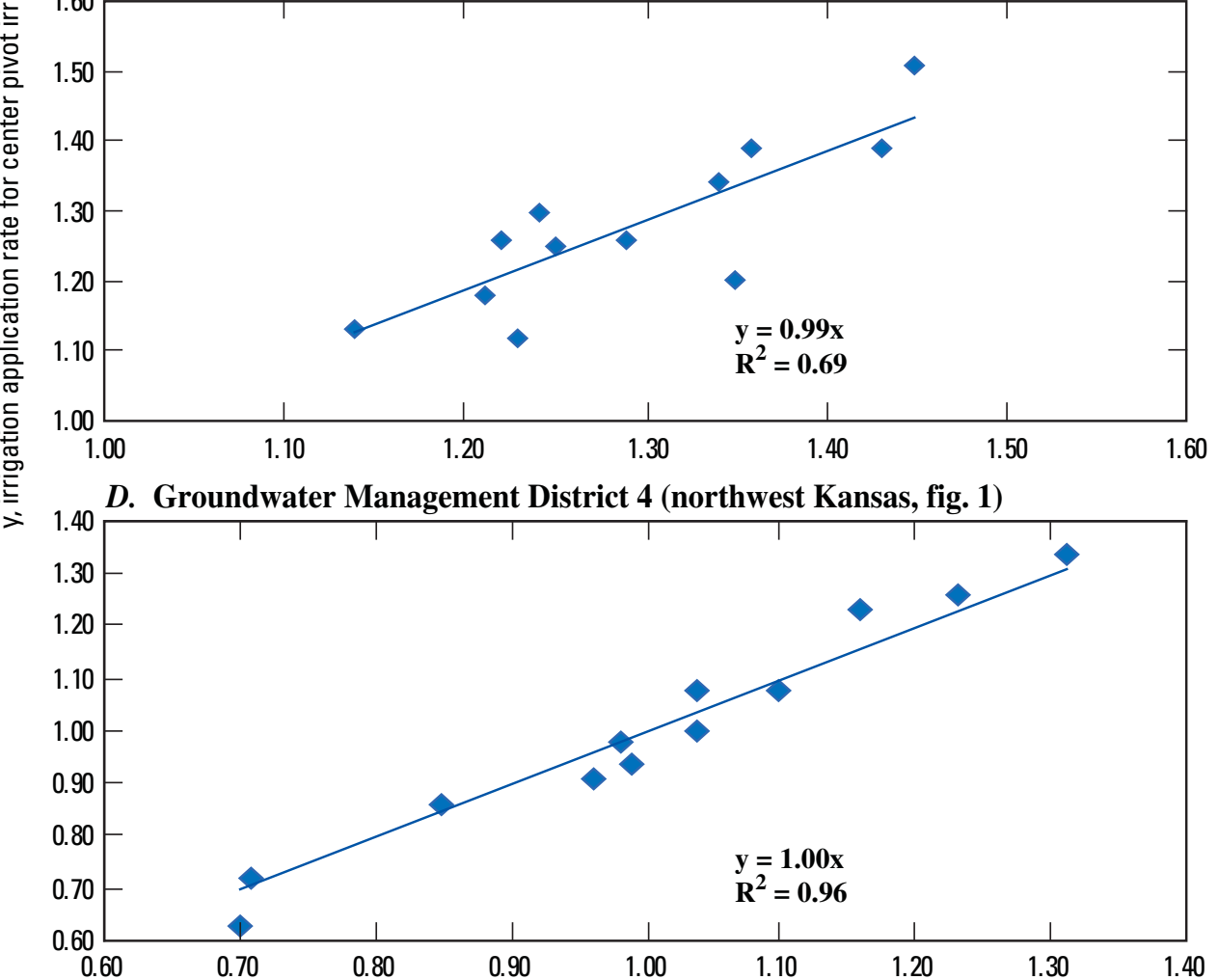

$x$, irrigation application rate for center pivot irrigation systems without drop nozzles, in acre-feet per acre

Figure 11. Relation between irrigation application rates for center pivot systems with and without drop nozzles for Groundwater Management Districts, 1992-2003. 


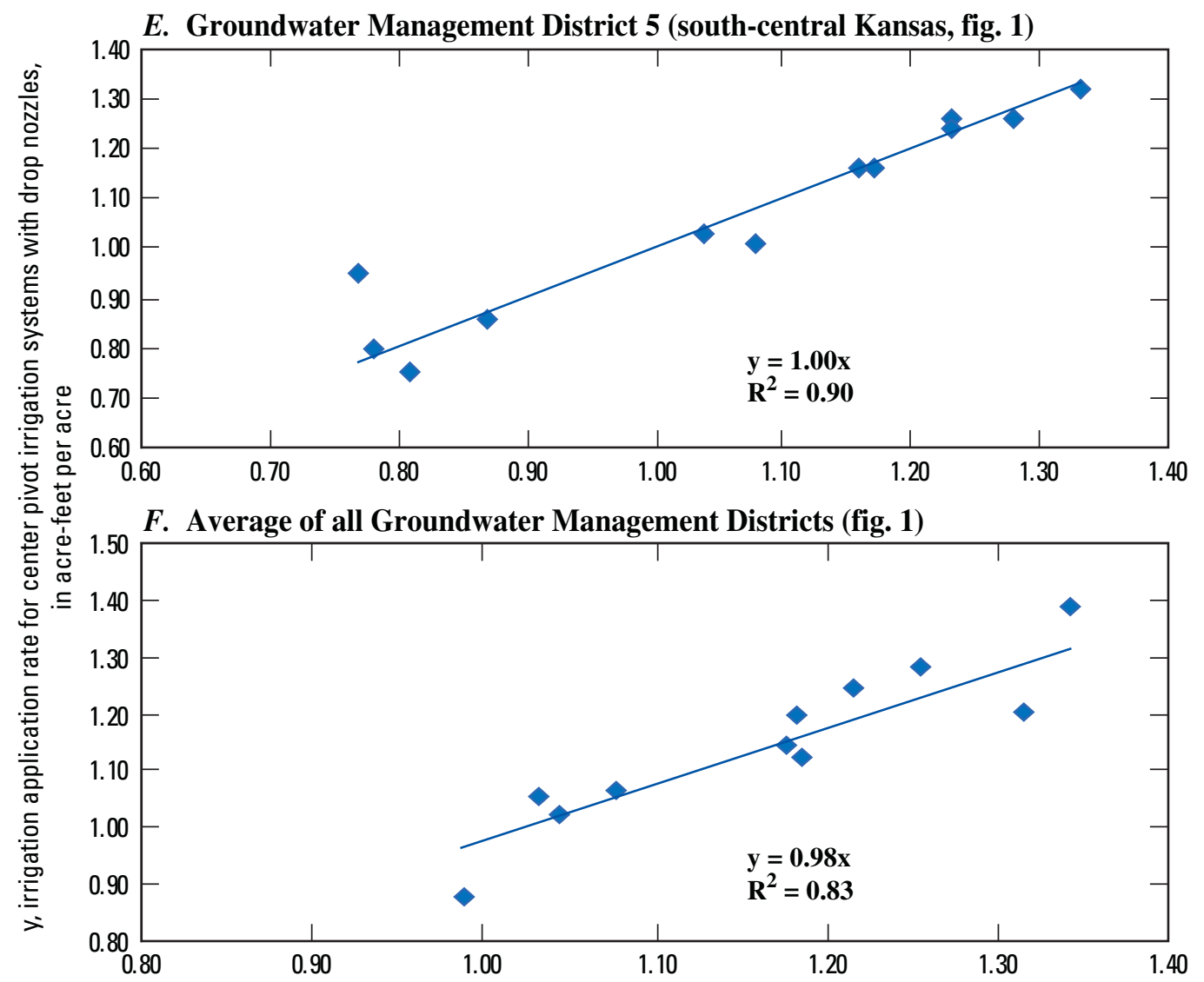

$\mathrm{x}$, irrigation application rate for center pivot irrigation systems without drop nozzles, in acre-feet per acre

Figure 11. Relation between irrigation application rates for center pivot systems with and without drop nozzles for Groundwater Management Districts, 1992-2003.—Continued 
A. Groundwater Management District 1 (west-central Kansas, fig. 1)
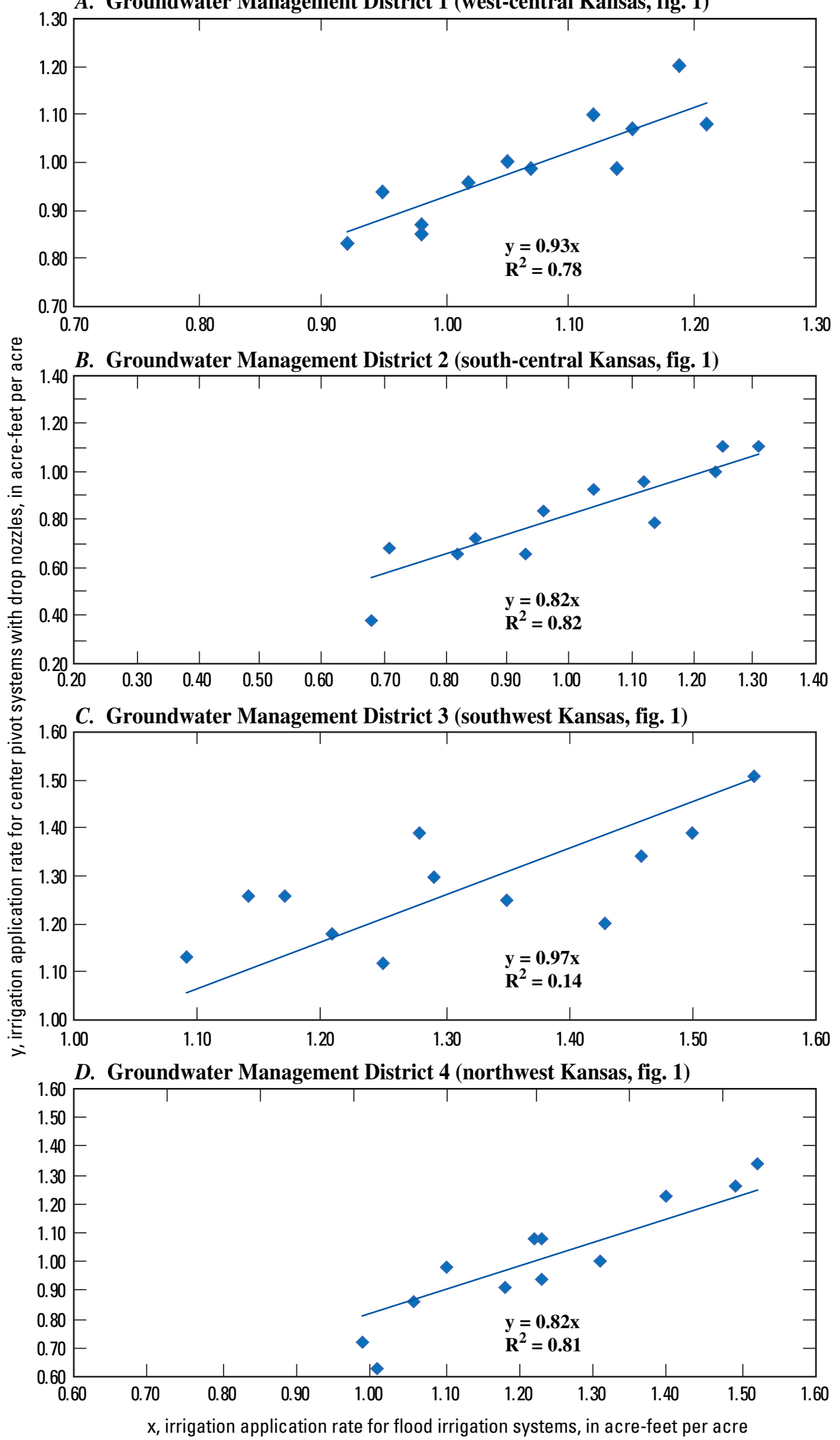

Figure 12. Relation between irrigation application rates for flood irrigation systems and center pivot systems with drop nozzles for Groundwater Management Districts, 1992-2003. 

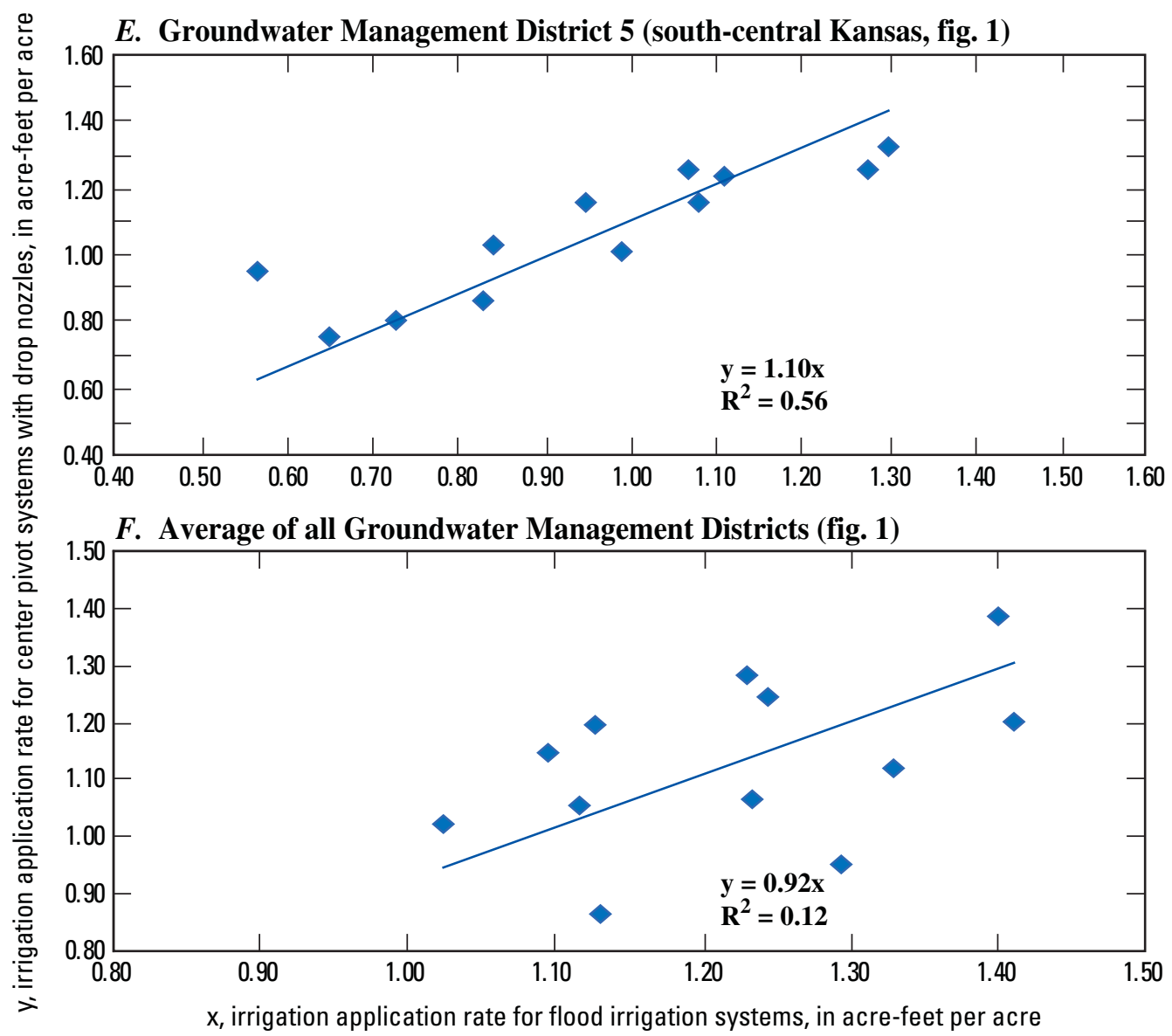

Figure 12. Relation between irrigation application rates for flood irrigation systems and center pivot systems with drop nozzles for Groundwater Management Districts, 1992-2003.-Continued

\section{References Cited}

Kansas State Climatologist, 2005, Precipitation summary, by county: Manhattan, Kansas State University Research and Extension, information available on Web, accessed October 6, 2005, at http://www.oznet.ksu.edu/wdl/wdl/ pmaps.htm

Kansas State Conservation Commission, 2005, FY 2003 SCC annual report: Topeka, Kansas, 27 p.

Kansas Water Office and Kansas Department of Agriculture, Division of Water Resources, 1993-2005, Kansas irrigation water use: Topeka, Kansas, published annually.

Kendall, M., and Gibbons, J.D., 1990, Rank correlation methods (5th ed.): New York, Oxford University Press, 260 p.
McGuire, V.L., 2001, Water-level changes in the High Plains aquifer, 1980 to 1999: U.S. Geological Survey Fact Sheet 029-01, 2 p.

McGuire, V.L., and Sharpe, J.B. 1997, Water-level changes in the High Plains aquifer-predevelopment to 1995: U.S. Geological Survey Water-Resources Investigations Report 974081, 2 sheets.

National Oceanic and Atmospheric Administration, 18952005, Climatological data, annual summary, Kansas, through volume 117: Ashville, North Carolina, National Climatic Center, published annually.

U.S. Department of Agriculture, 1994, State soil geographic (STATSGO) data base: Soil Conservation Miscellaneous Publication 1492, 37 p. 


\section{Data Tables}


Table 1. Precipitation, irrigation water use, acres irrigated, irrigation application rate by system type, and soil permeability for counties in the Groundwater

Management Districts within the Kansas High Plains, 1991-2003.

[Precipitation data from Kansas State Climatologist (2005). Irrigation data from Kansas Water Office and Kansas Department of Agriculture (1993-2005). Soil permeability from U.S. Department of Agriculture (1994). Blue shading indicates trend is significant at 95-percent confidence level ( $\mathrm{p} \leq 0.05)$. Green shading indicates trend is significant at 90 -percent confidence level ( $\mathrm{p} \leq 0.10)$. acre-ft, acre-feet; acre-ft/acre, acre-feet per acre; NA, data not available; --, not computed]

\begin{tabular}{|c|c|c|c|c|c|c|c|c|c|c|c|c|c|c|c|c|}
\hline \multirow[b]{2}{*}{ Year } & \multirow[b]{2}{*}{$\begin{array}{l}\text { Annual } \\
\text { precip- } \\
\text { itation } \\
\text { (inches) }\end{array}$} & \multirow[b]{2}{*}{$\begin{array}{l}\text { March- } \\
\text { October } \\
\text { precipi- } \\
\text { tation } \\
\text { (inches) }\end{array}$} & \multirow[b]{2}{*}{$\begin{array}{c}\text { Total } \\
\text { irrigation } \\
\text { water use } \\
\text { (acre-ft) }\end{array}$} & \multirow[b]{2}{*}{$\begin{array}{l}\text { Total acres } \\
\text { irrigated }\end{array}$} & \multicolumn{3}{|c|}{ Flood irrigation } & \multicolumn{3}{|c|}{ Center pivot irrigation } & \multicolumn{3}{|c|}{$\begin{array}{l}\text { Center pivot irrigation with } \\
\text { drop nozzle }\end{array}$} & \multicolumn{3}{|c|}{ Other irrigation systems } \\
\hline & & & & & $\begin{array}{c}\text { Total } \\
\text { acres } \\
\text { irrigated }\end{array}$ & $\begin{array}{c}\text { Water } \\
\text { used } \\
\text { (acre-ft) }\end{array}$ & $\begin{array}{c}\text { Rate of } \\
\text { irri- } \\
\text { gation } \\
\text { (acre- } \\
\text { ft/acre) }\end{array}$ & $\begin{array}{c}\text { Total } \\
\text { acres } \\
\text { irrigated }\end{array}$ & $\begin{array}{c}\text { Water } \\
\text { used } \\
\text { (acre-ft) }\end{array}$ & $\begin{array}{c}\text { Rate of } \\
\text { irri- } \\
\text { gation } \\
\text { (acre- } \\
\text { ft/acre) }\end{array}$ & $\begin{array}{c}\text { Total } \\
\text { acres } \\
\text { irrigated }\end{array}$ & $\begin{array}{c}\text { Water } \\
\text { used } \\
\text { (acre-ft) }\end{array}$ & $\begin{array}{l}\text { Rate of } \\
\text { irri- } \\
\text { gation } \\
\text { (acre- } \\
\text { ft/acre) }\end{array}$ & $\begin{array}{c}\text { Total } \\
\text { acres } \\
\text { irrigated }\end{array}$ & $\begin{array}{c}\text { Water } \\
\text { used } \\
\text { (acre-ft) }\end{array}$ & $\begin{array}{l}\text { Rate of } \\
\text { irri- } \\
\text { gation } \\
\text { (acre- } \\
\mathrm{ft} / \text { acre) }\end{array}$ \\
\hline
\end{tabular}

\begin{tabular}{|c|c|c|c|c|c|c|c|c|c|c|c|c|c|c|c|c|}
\hline \multicolumn{17}{|c|}{ Barton County (estimated mean soil permeability $=1.2$ inches per hour) } \\
\hline 1991 & 19.05 & 15.58 & 50,874 & 37,908 & 17,398 & 22,791 & 1.31 & 19,627 & 27,085 & 1.38 & NA & NA & NA & 883 & 997 & 1.13 \\
\hline 1992 & 32.59 & 27.28 & 20,220 & 32,508 & 11,892 & 5,827 & .49 & 18,745 & 13,496 & .72 & 1,364 & 709 & .52 & 507 & 187 & .37 \\
\hline 1993 & 38.22 & 32.97 & 17,959 & 31,882 & 12,137 & 6,554 & .54 & 18,900 & 10,962 & .58 & 428 & 223 & .52 & 417 & 220 & .53 \\
\hline 1994 & 18.00 & 15.04 & 43,754 & 35,425 & 14,731 & 17,825 & 1.21 & 19,292 & 24,308 & 1.26 & 737 & 921 & 1.25 & 665 & 700 & 1.05 \\
\hline 1995 & 26.55 & 25.38 & 33,487 & 36,004 & 15,547 & 13,837 & .89 & 19,001 & 18,241 & .96 & 914 & 960 & 1.05 & 542 & 450 & .83 \\
\hline 1996 & 31.52 & 28.74 & 33,203 & 36,416 & 14,864 & 13,080 & .88 & 19,865 & 18,077 & .91 & 1,170 & 1,310 & 1.12 & 517 & 735 & 1.42 \\
\hline 1997 & 26.36 & 22.94 & 24,952 & 36,395 & 14,099 & 8,459 & .60 & 14,978 & 10,934 & .73 & 6,962 & 5,361 & .77 & 356 & 198 & .56 \\
\hline 1998 & 29.69 & 23.58 & 30,239 & 36,235 & 13,526 & 10,145 & .75 & 14,587 & 13,274 & .91 & 7,677 & 6,525 & .85 & 445 & 295 & .66 \\
\hline 1999 & 28.59 & 25.66 & 28,049 & 36,867 & 13,945 & 9,622 & .69 & 14,042 & 11,936 & .85 & 8,455 & 6,172 & .73 & 425 & 319 & .75 \\
\hline 2000 & 29.32 & 24.67 & 38,111 & 37,538 & 13,014 & 12,624 & .97 & 12,556 & 13,184 & 1.05 & 10,374 & 10,893 & 1.05 & 1,594 & 1,411 & .88 \\
\hline 2001 & 29.66 & 24.57 & 39,885 & 36,665 & 13,387 & 12,985 & .97 & 10,933 & 12,573 & 1.15 & 11,660 & 13,292 & 1.14 & 685 & 1,034 & 1.51 \\
\hline 2002 & 19.01 & 17.77 & 40,168 & 38,204 & 12,598 & 11,968 & .95 & 6,917 & 7,678 & 1.11 & 16,438 & 18,082 & 1.10 & 2,251 & 2,440 & 1.08 \\
\hline 2003 & 21.32 & 18.28 & 42,814 & 37,675 & 13,300 & 14,630 & 1.10 & 5,248 & 5,563 & 1.06 & 18,276 & 21,931 & 1.20 & 851 & 690 & .81 \\
\hline Average & 26.91 & 23.26 & 34,132 & 36,132 & 13,880 & 12,334 & .87 & 14,976 & 14,409 & .97 & 7,038 & 7,198 & .94 & 780 & 744 & .95 \\
\hline p-value & .50 & -- & .30 & .01 & -- & -- & -- & -- & -- & -- & -- & -- & -- & -- & -- & -- \\
\hline Slope & -.33 & -- & 1,196 & 293 & -- & -- & -- & -- & -- & -- & -- & -- & -- & -- & -- & -- \\
\hline \multicolumn{17}{|c|}{ Cheyenne County (estimated mean soil permeability = 1.3 inches per hour) } \\
\hline 1991 & 24.99 & 22.38 & 56,064 & 43,979 & 13,981 & 20,133 & 1.44 & 25,190 & 28,969 & 1.15 & NA & NA & NA & 4,808 & 6,962 & 1.45 \\
\hline 1992 & 19.64 & 16.54 & 39,541 & 42,214 & 11,412 & 10,385 & .91 & 23,686 & 21,317 & .90 & 3,148 & 2,928 & .93 & 3,968 & 4,911 & 1.24 \\
\hline 1993 & 19.58 & 16.83 & 44,230 & 43,707 & 11,821 & 14,185 & 1.20 & 21,503 & 20,428 & .95 & 4,969 & 4,224 & .85 & 5,414 & 5,393 & 1.00 \\
\hline 1994 & 15.40 & 12.63 & 55,075 & 45,719 & 10,592 & 15,358 & 1.45 & 23,107 & 25,649 & 1.11 & 6,618 & 6,883 & 1.04 & 5,402 & 7,186 & 1.33 \\
\hline 1995 & 20.72 & 19.26 & 40,621 & 42,804 & 9,033 & 10,749 & 1.19 & 22,971 & 20,674 & .90 & 7,261 & 5,301 & .73 & 3,539 & 3,898 & 1.10 \\
\hline 1996 & 19.19 & 18.35 & 48,994 & 46,737 & 8,912 & 11,853 & 1.33 & 25,035 & 24,034 & .96 & 8,630 & 8,285 & .96 & 4,160 & 4,823 & 1.16 \\
\hline 1997 & 16.68 & 14.86 & 59,570 & 48,494 & 7,605 & 11,103 & 1.46 & 15,669 & 17,863 & 1.14 & 20,737 & 25,507 & 1.23 & 4,483 & 5,098 & 1.14 \\
\hline 1998 & 19.26 & 16.67 & 49,947 & 47,522 & 5,514 & 7,278 & 1.32 & 11,581 & 11,813 & 1.02 & 24,751 & 25,494 & 1.03 & 5,676 & 5,362 & .94 \\
\hline 1999 & 18.36 & 17.33 & 49,835 & 47,593 & 5,421 & 7,156 & 1.32 & 12,309 & 12,186 & .99 & 25,183 & 25,183 & 1.00 & 4,680 & 5,311 & 1.13 \\
\hline 2000 & 15.58 & 14.31 & 65,272 & 49,219 & 4,093 & 6,753 & 1.65 & 10,037 & 11,743 & 1.17 & 30,458 & 41,118 & 1.35 & 4,631 & 5,657 & 1.22 \\
\hline 2001 & 16.96 & 14.09 & 50,835 & 46,893 & 2,742 & 3,619 & 1.32 & 9,481 & 9,197 & .97 & 28,817 & 30,834 & 1.07 & 5,853 & 7,185 & 1.23 \\
\hline 2002 & 11.07 & 9.86 & 65,242 & 46,576 & 2,295 & 3,718 & 1.62 & 6,653 & 9,115 & 1.37 & 31,105 & 44,169 & 1.42 & 6,523 & 8,241 & 1.26 \\
\hline
\end{tabular}


Table 1. Precipitation, irrigation water use, acres irrigated, irrigation application rate by system type, and soil permeability for counties in the Groundwater Management Districts within the Kansas High Plains, 1991-2003.-Continued

[Precipitation data from Kansas State Climatologist (2005). Irrigation data from Kansas Water Office and Kansas Department of Agriculture (1993-2005). Soil permeability from U.S. Department of Agriculture (1994). Blue shading indicates trend is significant at 95-percent confidence level ( $\mathrm{p} \leq 0.05$ ). Green shading indicates trend is significant at 90 -percent confidence level ( $\mathrm{p} \leq 0.10$ ). acre-ft, acre-feet; acreft/acre, acre-feet per acre; NA, data not available; --, not computed]

\begin{tabular}{|c|c|c|c|c|c|c|c|c|c|c|c|c|c|c|c|c|}
\hline \multirow[b]{2}{*}{ Year } & \multirow[b]{2}{*}{$\begin{array}{l}\text { Annual } \\
\text { precip- } \\
\text { itation } \\
\text { (inches) }\end{array}$} & \multirow{2}{*}{$\begin{array}{l}\text { March- } \\
\text { October } \\
\text { precipi- } \\
\text { tation } \\
\text { (inches) }\end{array}$} & \multirow[b]{2}{*}{$\begin{array}{c}\text { Total } \\
\text { irrigation } \\
\text { water use } \\
\text { (acre-ft) }\end{array}$} & \multirow[b]{2}{*}{$\begin{array}{c}\text { Total acres } \\
\text { irrigated }\end{array}$} & \multicolumn{3}{|c|}{ Flood irrigation } & \multicolumn{3}{|c|}{ Center pivot irrigation } & \multicolumn{3}{|c|}{$\begin{array}{l}\text { Center pivot irrigation with } \\
\text { drop nozzle }\end{array}$} & \multicolumn{3}{|c|}{ Other irrigation systems } \\
\hline & & & & & $\begin{array}{c}\text { Total } \\
\text { acres } \\
\text { irrigated }\end{array}$ & $\begin{array}{c}\text { Water } \\
\text { used } \\
\text { (acre-ft) }\end{array}$ & $\begin{array}{l}\text { Rate of } \\
\text { irri- } \\
\text { gation } \\
\text { (acre- } \\
\text { ft/acre) }\end{array}$ & $\begin{array}{c}\text { Total } \\
\text { acres } \\
\text { irrigated }\end{array}$ & $\begin{array}{c}\text { Water } \\
\text { used } \\
\text { (acre-ft) }\end{array}$ & $\begin{array}{l}\text { Rate of } \\
\text { irri- } \\
\text { gation } \\
\text { (acre- } \\
\text { ft/acre) }\end{array}$ & $\begin{array}{c}\text { Total } \\
\text { acres } \\
\text { irrigated }\end{array}$ & $\begin{array}{c}\text { Water } \\
\text { used } \\
\text { (acre-ft) }\end{array}$ & $\begin{array}{l}\text { Rate of } \\
\text { irri- } \\
\text { gation } \\
\text { (acre- } \\
\text { ft/acre) }\end{array}$ & $\begin{array}{c}\text { Total } \\
\text { acres } \\
\text { irrigated }\end{array}$ & $\begin{array}{c}\text { Water } \\
\text { used } \\
\text { (acre-ft) }\end{array}$ & $\begin{array}{c}\text { Rate of } \\
\text { irri- } \\
\text { gation } \\
\text { (acre- } \\
\text { ft/acre) }\end{array}$ \\
\hline \multicolumn{17}{|c|}{ Cheyenne County (estimated mean soil permeability $=1.3$ inches per hour) - Continued } \\
\hline 2003 & 13.65 & 12.01 & 57,683 & 46,586 & 1,722 & 2,325 & 1.35 & 8,123 & 9,585 & 1.18 & 32,390 & 40,811 & 1.26 & 4,351 & 4,962 & 1.14 \\
\hline Average & 17.78 & 15.78 & 52,531 & 46,003 & 7,319 & 9,586 & 1.35 & 16,565 & 17,121 & 1.06 & 18,672 & 21,728 & 1.07 & 4,884 & 5,768 & 1.18 \\
\hline p-value & 0 & -- & .06 & .06 & -- & -- & -- & -- & -- & -- & -- & -- & -- & -- & -- & -- \\
\hline slope & -.67 & -- & 1,248 & 395 & -- & -- & -- & -- & -- & -- & -- & -- & -- & -- & -- & -- \\
\hline \multicolumn{17}{|c|}{ Edwards County (estimated mean soil permeability $=3.9$ inches per hour) } \\
\hline 1991 & 22.44 & 19.46 & 134,667 & 94,833 & 4,777 & 7,834 & 1.64 & 89,643 & 126,397 & 1.41 & NA & NA & NA & 413 & 436 & 1.06 \\
\hline 1992 & 33.17 & 27.72 & 79,543 & 94,801 & 4,196 & 3,525 & .84 & 86,595 & 73,606 & .85 & 3,377 & 2,263 & .67 & 633 & 150 & .24 \\
\hline 1993 & 32.52 & 27.05 & 75,378 & 92,703 & 3,935 & 3,187 & .81 & 86,289 & 69,894 & .81 & 1,909 & 2,176 & 1.14 & 570 & 121 & .21 \\
\hline 1994 & 18.87 & 15.59 & 124,957 & 93,019 & 2,871 & 4,393 & 1.53 & 88,052 & 117,990 & 1.34 & 1,325 & 1,776 & 1.34 & 771 & 800 & 1.04 \\
\hline 1995 & 30.08 & 28.15 & 104,652 & 92,835 & 2,517 & 3,373 & 1.34 & 86,430 & 96,802 & 1.12 & 2,974 & 3,450 & 1.16 & 914 & 1,028 & 1.12 \\
\hline 1996 & 37.37 & 34.80 & 74,959 & 94,891 & 2,659 & 2,047 & .77 & 88,029 & 69,543 & .79 & 3,052 & 2,289 & .75 & 1,151 & 1,079 & .94 \\
\hline 1997 & 36.85 & 31.67 & 77,679 & 96,756 & 2,044 & 1,880 & .92 & 74,943 & 60,704 & .81 & 19,582 & 14,882 & .76 & 187 & 212 & 1.13 \\
\hline 1998 & 24.22 & 19.43 & 126,379 & 97,971 & 1,780 & 1,887 & 1.06 & 59,543 & 77,406 & 1.30 & 31,238 & 39,360 & 1.26 & 5,410 & 7,726 & 1.43 \\
\hline 1999 & 25.11 & 22.16 & 108,909 & 100,065 & 1,885 & 2,055 & 1.09 & 58,763 & 65,227 & 1.11 & 35,849 & 38,000 & 1.06 & 3,568 & 3,628 & 1.02 \\
\hline 2000 & 30.27 & 27.08 & 126,017 & 100,031 & 1,942 & 2,486 & 1.28 & 55,352 & 70,297 & 1.27 & 40,791 & 50,581 & 1.24 & 1,946 & 2,654 & 1.36 \\
\hline 2001 & 24.34 & 20.55 & 127,470 & 99,786 & 1,771 & 2,107 & 1.19 & 43,471 & 55,643 & 1.28 & 46,696 & 59,771 & 1.28 & 7,848 & 9,949 & 1.27 \\
\hline 2002 & 18.92 & 16.85 & 134,264 & 101,294 & 1,553 & 2,159 & 1.39 & 33,965 & 43,815 & 1.29 & 64,352 & 86,875 & 1.35 & 1,424 & 1,415 & .99 \\
\hline 2003 & 19.24 & 17.27 & 127,702 & 100,527 & 1,231 & 1,822 & 1.48 & 26,200 & 33,274 & 1.27 & 69,309 & 87,329 & 1.26 & 3,787 & 5,277 & 1.39 \\
\hline Average & 27.18 & 23.68 & 109,429 & 96,886 & 2,551 & 2,981 & 1.18 & 67,483 & 73,892 & 1.13 & 26,705 & 32,396 & 1.11 & 2,202 & 2,652 & 1.20 \\
\hline p-value & .29 & -- & .10 & 0 & -- & -- & -- & -- & -- & -- & -- & -- & -- & -- & -- & -- \\
\hline slope & -.99 & -- & 2,800 & 754 & -- & -- & -- & -- & -- & -- & -- & -- & -- & -- & -- & -- \\
\hline \multicolumn{17}{|c|}{ Finney County (estimated mean soil permeability 1.9 inches per hour) } \\
\hline 1991 & 19.50 & 16.72 & 408,799 & 253,357 & 111,485 & 179,491 & 1.61 & 130,393 & 212,541 & 1.63 & NA & NA & NA & 11,479 & 16,768 & 1.46 \\
\hline 1992 & 23.63 & 19.10 & 289,956 & 250,194 & 101,956 & 103,995 & 1.02 & 128,806 & 168,736 & 1.31 & 4,741 & 4,836 & 1.02 & 14,691 & 12,390 & .84 \\
\hline 1993 & 26.75 & 22.30 & 266,562 & 241,411 & 93,313 & 83,982 & .90 & 118,936 & 149,859 & 1.26 & 11,946 & 17,800 & 1.49 & 17,216 & 14,921 & .87 \\
\hline 1994 & 17.85 & 15.47 & 348,399 & 243,085 & 89,163 & 114,129 & 1.28 & 117,657 & 183,545 & 1.56 & 16,478 & 28,013 & 1.70 & 19,787 & 22,712 & 1.15 \\
\hline 1995 & 20.73 & 19.23 & 309,058 & 243,298 & 76,362 & 87,816 & 1.15 & 125,751 & 172,279 & 1.37 & 20,576 & 28,395 & 1.38 & 20,609 & 20,568 & 1.00 \\
\hline 1996 & 22.74 & 21.06 & 296,412 & 240,283 & 74,761 & 88,218 & 1.18 & 118,068 & 149,946 & 1.27 & 30,627 & 41,040 & 1.34 & 16,827 & 17,208 & 1.02 \\
\hline 1997 & 25.84 & 22.97 & 292,861 & 243,130 & 65,394 & 71,279 & 1.09 & 84,582 & 115,032 & 1.36 & 72,802 & 86,634 & 1.19 & 20,352 & 19,915 & .98 \\
\hline
\end{tabular}


Table 1. Precipitation, irrigation water use, acres irrigated, irrigation application rate by system type, and soil permeability for counties in the Groundwater Management Districts within the Kansas High Plains, 1991-2003.-Continued

[Precipitation data from Kansas State Climatologist (2005). Irrigation data from Kansas Water Office and Kansas Department of Agriculture (1993-2005). Soil permeability from U.S. Department of Agriculture (1994). Blue shading indicates trend is significant at 95 -percent confidence level ( $\mathrm{p} \leq 0.05$ ). Green shading indicates trend is significant at 90 -percent confidence level ( $\mathrm{p} \leq 0.10)$. acre-ft, acre-feet; acreft/acre, acre-feet per acre; NA, data not available; --, not computed]

\begin{tabular}{|c|c|c|c|c|c|c|c|c|c|c|c|c|c|c|c|c|}
\hline \multirow[b]{2}{*}{ Year } & \multirow[b]{2}{*}{$\begin{array}{l}\text { Annual } \\
\text { precip- } \\
\text { itation } \\
\text { (inches) }\end{array}$} & \multirow{2}{*}{$\begin{array}{l}\text { March- } \\
\text { October } \\
\text { precipi- } \\
\text { tation } \\
\text { (inches) }\end{array}$} & \multirow[b]{2}{*}{$\begin{array}{c}\text { Total } \\
\text { irrigation } \\
\text { water use } \\
\text { (acre-ft) }\end{array}$} & \multirow[b]{2}{*}{$\begin{array}{c}\text { Total acres } \\
\text { irrigated }\end{array}$} & \multicolumn{3}{|c|}{ Flood irrigation } & \multicolumn{3}{|c|}{ Center pivot irrigation } & \multicolumn{3}{|c|}{$\begin{array}{l}\text { Center pivot irrigation with } \\
\text { drop nozzle }\end{array}$} & \multicolumn{3}{|c|}{ Other irrigation systems } \\
\hline & & & & & $\begin{array}{c}\text { Total } \\
\text { acres } \\
\text { irrigated }\end{array}$ & $\begin{array}{c}\text { Water } \\
\text { used } \\
\text { (acre-ft) }\end{array}$ & $\begin{array}{l}\text { Rate of } \\
\text { irri- } \\
\text { gation } \\
\text { (acre- } \\
\text { ft/acre) }\end{array}$ & $\begin{array}{c}\text { Total } \\
\text { acres } \\
\text { irrigated }\end{array}$ & $\begin{array}{c}\text { Water } \\
\text { used } \\
\text { (acre-ft) }\end{array}$ & $\begin{array}{l}\text { Rate of } \\
\text { irri- } \\
\text { gation } \\
\text { (acre- } \\
\text { ft/acre) }\end{array}$ & $\begin{array}{c}\text { Total } \\
\text { acres } \\
\text { irrigated }\end{array}$ & $\begin{array}{c}\text { Water } \\
\text { used } \\
\text { (acre-ft) }\end{array}$ & $\begin{array}{l}\text { Rate of } \\
\text { irri- } \\
\text { gation } \\
\text { (acre- } \\
\text { ft/acre) }\end{array}$ & $\begin{array}{c}\text { Total } \\
\text { acres } \\
\text { irrigated }\end{array}$ & $\begin{array}{c}\text { Water } \\
\text { used } \\
\text { (acre-ft) }\end{array}$ & $\begin{array}{c}\text { Rate of } \\
\text { irri- } \\
\text { gation } \\
\text { (acre- } \\
\text { ft/acre) }\end{array}$ \\
\hline \multicolumn{17}{|c|}{ Finney County (estimated mean soil permeability 1.9 inches per hour)-Continued } \\
\hline 1998 & 21.36 & 17.98 & 295,060 & 240,956 & 59,493 & 53,544 & 0.90 & 83,663 & 117,128 & 1.40 & 73,783 & 97,394 & 1.32 & 24,017 & 26,995 & 1.12 \\
\hline 1999 & 22.29 & 20.70 & 263,257 & 241,634 & 54,534 & 46,899 & .86 & 64,587 & 82,025 & 1.27 & 95,227 & 108,559 & 1.14 & 27,286 & 25,774 & .94 \\
\hline 2000 & 19.01 & 17.71 & 321,831 & 236,280 & 51,426 & 53,997 & 1.05 & 54,882 & 86,714 & 1.58 & 102,468 & 150,628 & 1.47 & 27,504 & 30,492 & 1.11 \\
\hline 2001 & 18.83 & 17.06 & 284,764 & 241,713 & 45,689 & 39,749 & .87 & 35,544 & 45,852 & 1.29 & 128,058 & 165,195 & 1.29 & 32,422 & 33,968 & 1.05 \\
\hline 2002 & 14.04 & 13.04 & 369,960 & 244,296 & 45,673 & 68,510 & 1.50 & 31,908 & 48,181 & 1.51 & 132,503 & 206,705 & 1.56 & 34,212 & 46,565 & 1.36 \\
\hline 2003 & 16.09 & 14.78 & 322,310 & 235,065 & 34,438 & 45,803 & 1.33 & 33,794 & 47,650 & 1.41 & 129,857 & 184,397 & 1.42 & 36,976 & 44,461 & 1.20 \\
\hline Average & 20.67 & 18.31 & 313,018 & 242,669 & 69,514 & 79,801 & 1.13 & 86,813 & 121,499 & 1.40 & 68,256 & 93,300 & 1.36 & 23,337 & 25,595 & 1.10 \\
\hline p-value & .06 & -- & 1.0 & .08 & -- & -- & -- & -- & -- & -- & -- & -- & -- & -- & -- & -- \\
\hline slope & -.56 & -- & -195 & -764 & -- & -- & -- & -- & -- & -- & -- & -- & -- & -- & -- & -- \\
\hline \multicolumn{17}{|c|}{ Ford County (estimated mean soil permeability = 2.5 inches per hour) } \\
\hline 1991 & 13.50 & 10.40 & 141,455 & 93,300 & 18,433 & 33,179 & 1.80 & 65,182 & 95,166 & 1.46 & NA & NA & NA & 9,685 & 13,110 & 1.35 \\
\hline 1992 & 25.41 & 21.30 & 92,433 & 89,827 & 16,955 & 22,211 & 1.31 & 60,888 & 57,844 & .95 & 5,505 & 5,890 & 1.07 & 6,479 & 6,488 & 1.00 \\
\hline 1993 & 25.66 & 20.68 & 88,526 & 89,023 & 14,700 & 17,640 & 1.20 & 61,536 & 58,459 & .95 & 3,762 & 4,138 & 1.10 & 9,025 & 8,288 & .92 \\
\hline 1994 & 19.28 & 16.57 & 111,336 & 91,698 & 13,971 & 19,140 & 1.37 & 63,671 & 74,495 & 1.17 & 4,478 & 5,642 & 1.26 & 9,578 & 12,058 & 1.26 \\
\hline 1995 & 23.54 & 22.06 & 100,312 & 89,654 & 12,183 & 15,594 & 1.28 & 62,550 & 66,303 & 1.06 & 5,268 & 7,270 & 1.38 & 9,653 & 11,145 & 1.15 \\
\hline 1996 & 33.56 & 30.55 & 82,198 & 89,347 & 9,728 & 8,172 & .84 & 63,224 & 58,166 & .92 & 6,617 & 6,485 & .98 & 9,778 & 9,376 & .96 \\
\hline 1997 & 28.49 & 24.68 & 74,438 & 86,938 & 7,309 & 5,482 & .75 & 51,306 & 43,097 & .84 & 21,995 & 19,576 & .89 & 6,328 & 6,284 & .99 \\
\hline 1998 & 22.20 & 18.33 & 99,973 & 86,708 & 6,111 & 6,844 & 1.12 & 37,098 & 42,663 & 1.15 & 32,984 & 38,921 & 1.18 & 10,515 & 11,545 & 1.10 \\
\hline 1999 & 21.84 & 19.83 & 89,491 & 87,610 & 4,985 & 5,135 & 1.03 & 29,366 & 29,953 & 1.02 & 42,917 & 45,063 & 1.05 & 10,342 & 9,340 & .90 \\
\hline 2000 & 23.38 & 21.62 & 104,815 & 90,356 & 4,914 & 6,044 & 1.23 & 29,072 & 32,851 & 1.13 & 45,250 & 54,753 & 1.21 & 11,120 & 11,167 & 1.00 \\
\hline 2001 & 17.50 & 14.55 & 107,713 & 91,476 & 4,281 & 5,094 & 1.19 & 27,015 & 31,878 & 1.18 & 48,628 & 58,840 & 1.21 & 11,552 & 11,901 & 1.03 \\
\hline 2002 & 17.84 & 16.32 & 115,809 & 93,396 & 3,918 & 5,328 & 1.36 & 21,162 & 24,125 & 1.14 & 55,977 & 71,091 & 1.27 & 12,339 & 15,265 & 1.24 \\
\hline 2003 & 19.07 & 17.19 & 98,452 & 90,029 & 3,352 & 3,553 & 1.06 & 22,640 & 24,225 & 1.07 & 54,788 & 61,910 & 1.13 & 9,249 & 8,764 & .95 \\
\hline Average & 22.40 & 19.54 & 100,535 & 89,951 & 9,295 & 11,801 & 1.20 & 45,747 & 49,171 & 1.08 & 27,347 & 31,632 & 1.14 & 9,665 & 10,364 & 1.07 \\
\hline $\mathrm{p}$-value & .30 & -- & .05 & 1.0 & -- & -- & -- & -- & -- & -- & -- & -- & -- & -- & -- & -- \\
\hline slope & -.48 & -- & 553 & -11 & -- & -- & -- & -- & -- & -- & -- & -- & -- & -- & -- & -- \\
\hline
\end{tabular}


Table 1. Precipitation, irrigation water use, acres irrigated, irrigation application rate by system type, and soil permeability for counties in the Groundwater Management Districts within the Kansas High Plains, 1991-2003.-Continued

[Precipitation data from Kansas State Climatologist (2005). Irrigation data from Kansas Water Office and Kansas Department of Agriculture (1993-2005). Soil permeability from U.S. Department of Agriculture (1994). Blue shading indicates trend is significant at 95-percent confidence level ( $\mathrm{p} \leq 0.05)$. Green shading indicates trend is significant at 90-percent confidence level ( $\mathrm{p} \leq 0.10)$. acre-ft, acre-feet; acreft/acre, acre-feet per acre; NA, data not available; --, not computed]

\begin{tabular}{|c|c|c|c|c|c|c|c|c|c|c|c|c|c|c|c|c|}
\hline \multirow[b]{2}{*}{ Year } & \multirow[b]{2}{*}{$\begin{array}{l}\text { Annual } \\
\text { precip- } \\
\text { itation } \\
\text { (inches) }\end{array}$} & \multirow{2}{*}{$\begin{array}{l}\text { March- } \\
\text { October } \\
\text { precipi- } \\
\text { tation } \\
\text { (inches) }\end{array}$} & \multirow[b]{2}{*}{$\begin{array}{c}\text { Total } \\
\text { irrigation } \\
\text { water use } \\
\text { (acre-ft) }\end{array}$} & \multirow[b]{2}{*}{$\begin{array}{c}\text { Total acres } \\
\text { irrigated }\end{array}$} & \multicolumn{3}{|c|}{ Flood irrigation } & \multicolumn{3}{|c|}{ Center pivot irrigation } & \multicolumn{3}{|c|}{$\begin{array}{l}\text { Center pivot irrigation with } \\
\text { drop nozzle }\end{array}$} & \multicolumn{3}{|c|}{ Other irrigation systems } \\
\hline & & & & & $\begin{array}{c}\text { Total } \\
\text { acres } \\
\text { irrigated }\end{array}$ & $\begin{array}{c}\text { Water } \\
\text { used } \\
\text { (acre-ft) }\end{array}$ & $\begin{array}{c}\text { Rate of } \\
\text { irri- } \\
\text { gation } \\
\text { (acre- } \\
\text { ft/acre) }\end{array}$ & $\begin{array}{c}\text { Total } \\
\text { acres } \\
\text { irrigated }\end{array}$ & $\begin{array}{c}\text { Water } \\
\text { used } \\
\text { (acre-ft) }\end{array}$ & $\begin{array}{l}\text { Rate of } \\
\text { irri- } \\
\text { gation } \\
\text { (acre- } \\
\text { ft/acre) }\end{array}$ & $\begin{array}{c}\text { Total } \\
\text { acres } \\
\text { irrigated }\end{array}$ & $\begin{array}{c}\text { Water } \\
\text { used } \\
\text { (acre-ft) }\end{array}$ & $\begin{array}{c}\text { Rate of } \\
\text { irri- } \\
\text { gation } \\
\text { (acre- } \\
\text { ft/acre) }\end{array}$ & $\begin{array}{c}\text { Total } \\
\text { acres } \\
\text { irrigated }\end{array}$ & $\begin{array}{c}\text { Water } \\
\text { used } \\
\text { (acre-ft) }\end{array}$ & $\begin{array}{l}\text { Rate of } \\
\text { irri- } \\
\text { gation } \\
\text { (acre- } \\
\text { ft/acre) }\end{array}$ \\
\hline \multicolumn{17}{|c|}{ Graham County (estimated mean soil permeability $=1.5$ inches per hour) } \\
\hline 1991 & 20.15 & 16.69 & 15,629 & 12,339 & 2,431 & 4,522 & 1.86 & 8,021 & 8,663 & 1.08 & NA & NA & NA & 1,887 & 2,445 & 1.30 \\
\hline 1992 & 25.27 & 20.80 & 6,632 & 11,164 & 1,163 & 1,093 & .94 & 7,514 & 4,058 & .54 & 1,011 & 536 & .53 & 1,476 & 945 & .64 \\
\hline 1993 & 37.58 & 33.44 & 3,122 & 8,888 & 939 & 714 & .76 & 7,024 & 1,967 & .28 & 383 & 153 & .40 & 542 & 288 & .53 \\
\hline 1994 & 16.27 & 12.23 & 10,275 & 11,312 & 1,315 & 1,854 & 1.41 & 8,288 & 6,962 & .84 & 762 & 686 & .90 & 947 & 774 & .82 \\
\hline 1995 & 25.96 & 24.05 & 10,806 & 11,374 & 939 & 1,268 & 1.35 & 8,340 & 7,673 & .92 & 1,282 & 1,051 & .82 & 813 & 815 & 1.00 \\
\hline 1996 & 26.44 & 23.19 & 9,708 & 12,164 & 962 & 1,126 & 1.17 & 8,793 & 6,683 & .76 & 1,596 & 1,181 & .74 & 813 & 719 & .88 \\
\hline 1997 & 22.51 & 19.12 & 11,448 & 12,604 & 859 & 1,220 & 1.42 & 6,874 & 5,912 & .86 & 4,338 & 3,948 & .91 & 533 & 369 & .69 \\
\hline 1998 & 23.74 & 19.41 & 11,531 & 13,764 & 564 & 778 & 1.38 & 6,524 & 5,219 & .80 & 5,895 & 5,070 & .86 & 781 & 463 & .59 \\
\hline 1999 & 20.92 & 19.64 & 9,626 & 13,838 & 515 & 659 & 1.28 & 6,819 & 4,705 & .69 & 5,529 & 3,649 & .66 & 975 & 612 & .63 \\
\hline 2000 & 20.00 & 17.32 & 14,681 & 14,590 & 659 & 1,061 & 1.61 & 6,661 & 6,128 & .92 & 6,803 & 7,075 & 1.04 & 467 & 417 & .89 \\
\hline 2001 & 22.35 & 18.94 & 11,972 & 14,098 & 669 & 896 & 1.34 & 5,609 & 4,487 & .80 & 7,085 & 5,810 & .82 & 735 & 779 & 1.06 \\
\hline 2002 & 14.40 & 13.55 & 17,127 & 14,774 & 479 & 666 & 1.39 & 4,347 & 4,434 & 1.02 & 9,139 & 10,784 & 1.18 & 809 & 1,243 & 1.54 \\
\hline 2003 & 13.65 & 12.39 & 15,383 & 14,759 & 428 & 496 & 1.16 & 4,512 & 4,422 & .98 & 8,649 & 9,254 & 1.07 & 1,170 & 1,210 & 1.03 \\
\hline Average & 22.25 & 19.29 & 11,380 & 12,744 & 917 & 1,258 & 1.31 & 6,871 & 5,485 & .81 & 4,373 & 4,100 & .83 & 919 & 852 & .93 \\
\hline p-value & .04 & -- & .03 & 0 & -- & -- & -- & -- & -- & -- & -- & -- & -- & -- & -- & -- \\
\hline slope & -.83 & -- & 695 & 392 & -- & -- & -- & -- & -- & -- & -- & -- & -- & -- & -- & -- \\
\hline \multicolumn{17}{|c|}{ Grant County (estimated mean soil permeability $=2.4$ inches per hour) } \\
\hline 1991 & 19.36 & 16.62 & 236,163 & 142,038 & 86,579 & 139,392 & 1.61 & 37,394 & 65,440 & 1.75 & NA & NA & NA & 18,065 & 31,331 & 1.73 \\
\hline 1992 & 11.79 & 9.20 & 212,451 & 143,886 & 84,177 & 122,057 & 1.45 & 36,900 & 58,302 & 1.58 & 9,136 & 12,973 & 1.42 & 13,673 & 19,120 & 1.40 \\
\hline 1993 & 22.60 & 18.81 & 158,507 & 138,752 & 67,988 & 74,787 & 1.10 & 41,424 & 54,680 & 1.32 & 8,413 & 8,750 & 1.04 & 20,927 & 20,291 & .97 \\
\hline 1994 & 17.36 & 15.90 & 196,856 & 139,096 & 58,423 & 82,376 & 1.41 & 41,965 & 62,948 & 1.50 & 11,676 & 14,829 & 1.27 & 27,032 & 36,704 & 1.36 \\
\hline 1995 & 15.45 & 15.20 & 186,807 & 136,205 & 45,898 & 65,175 & 1.42 & 44,565 & 61,945 & 1.39 & 12,453 & 15,691 & 1.26 & 33,289 & 43,996 & 1.32 \\
\hline 1996 & 22.56 & 21.32 & 172,941 & 134,228 & 42,468 & 54,784 & 1.29 & 44,736 & 61,736 & 1.38 & 11,824 & 13,243 & 1.12 & 35,200 & 43,178 & 1.23 \\
\hline 1997 & 19.29 & 18.55 & 144,617 & 137,703 & 32,925 & 32,925 & 1.00 & 29,835 & 34,012 & 1.14 & 35,910 & 38,783 & 1.08 & 39,033 & 38,897 & 1.00 \\
\hline 1998 & 16.57 & 14.65 & 143,152 & 134,983 & 24,581 & 21,385 & .87 & 26,822 & 33,259 & 1.24 & 40,744 & 48,485 & 1.19 & 42,836 & 40,021 & .93 \\
\hline 1999 & 15.44 & 13.97 & 144,243 & 130,566 & 18,429 & 17,508 & .95 & 25,199 & 32,759 & 1.30 & 45,725 & 50,755 & 1.11 & 41,213 & 43,222 & 1.05 \\
\hline 2000 & 18.50 & 17.75 & 167,867 & 126,929 & 11,195 & 11,419 & 1.02 & 25,795 & 37,661 & 1.46 & 50,422 & 71,599 & 1.42 & 39,517 & 47,188 & 1.19 \\
\hline 2001 & 16.91 & 16.04 & 121,369 & 119,984 & 7,202 & 5,762 & .80 & 20,825 & 22,283 & 1.07 & 51,413 & 57,068 & 1.11 & 40,544 & 36,256 & .89 \\
\hline 2002 & 11.75 & 10.86 & 166,915 & 120,861 & 6,089 & 7,611 & 1.25 & 14,604 & 19,715 & 1.35 & 65,494 & 95,621 & 1.46 & 34,674 & 43,967 & 1.27 \\
\hline
\end{tabular}


Table 1. Precipitation, irrigation water use, acres irrigated, irrigation application rate by system type, and soil permeability for counties in the Groundwater Management Districts within the Kansas High Plains, 1991-2003.-Continued

[Precipitation data from Kansas State Climatologist (2005). Irrigation data from Kansas Water Office and Kansas Department of Agriculture (1993-2005). Soil permeability from U.S. Department of Agriculture (1994). Blue shading indicates trend is significant at 95 -percent confidence level ( $\mathrm{p} \leq 0.05$ ). Green shading indicates trend is significant at 90 -percent confidence level ( $\mathrm{p} \leq 0.10)$. acre-ft, acre-feet; acreft/acre, acre-feet per acre; NA, data not available; --, not computed]

\begin{tabular}{|c|c|c|c|c|c|c|c|c|c|c|c|c|c|c|c|c|}
\hline \multirow[b]{2}{*}{ Year } & \multirow[b]{2}{*}{$\begin{array}{l}\text { Annual } \\
\text { precip- } \\
\text { itation } \\
\text { (inches) }\end{array}$} & \multirow{2}{*}{$\begin{array}{l}\text { March- } \\
\text { October } \\
\text { precipi- } \\
\text { tation } \\
\text { (inches) }\end{array}$} & \multirow[b]{2}{*}{$\begin{array}{c}\text { Total } \\
\text { irrigation } \\
\text { water use } \\
\text { (acre-ft) }\end{array}$} & \multirow[b]{2}{*}{$\begin{array}{c}\text { Total acres } \\
\text { irrigated }\end{array}$} & \multicolumn{3}{|c|}{ Flood irrigation } & \multicolumn{3}{|c|}{ Center pivot irrigation } & \multicolumn{3}{|c|}{$\begin{array}{l}\text { Center pivot irrigation with } \\
\text { drop nozzle }\end{array}$} & \multicolumn{3}{|c|}{ Other irrigation systems } \\
\hline & & & & & $\begin{array}{c}\text { Total } \\
\text { acres } \\
\text { irrigated }\end{array}$ & $\begin{array}{c}\text { Water } \\
\text { used } \\
\text { (acre-ft) }\end{array}$ & $\begin{array}{l}\text { Rate of } \\
\text { irri- } \\
\text { gation } \\
\text { (acre- } \\
\text { ft/acre) }\end{array}$ & $\begin{array}{c}\text { Total } \\
\text { acres } \\
\text { irrigated }\end{array}$ & $\begin{array}{c}\text { Water } \\
\text { used } \\
\text { (acre-ft) }\end{array}$ & $\begin{array}{l}\text { Rate of } \\
\text { irri- } \\
\text { gation } \\
\text { (acre- } \\
\text { ft/acre) }\end{array}$ & $\begin{array}{c}\text { Total } \\
\text { acres } \\
\text { irrigated }\end{array}$ & $\begin{array}{c}\text { Water } \\
\text { used } \\
\text { (acre-ft) }\end{array}$ & $\begin{array}{l}\text { Rate of } \\
\text { irri- } \\
\text { gation } \\
\text { (acre- } \\
\text { ft/acre) }\end{array}$ & $\begin{array}{c}\text { Total } \\
\text { acres } \\
\text { irrigated }\end{array}$ & $\begin{array}{c}\text { Water } \\
\text { used } \\
\text { (acre-ft) }\end{array}$ & $\begin{array}{c}\text { Rate of } \\
\text { irri- } \\
\text { gation } \\
\text { (acre- } \\
\text { ft/acre) }\end{array}$ \\
\hline \multicolumn{17}{|c|}{ Grant County (estimated mean soil permeability $=2.4$ inches per hour) - Continued } \\
\hline 2003 & 15.32 & 14.33 & 144,167 & 117,232 & 4,551 & 4,278 & 0.94 & 14,137 & 17,954 & 1.27 & 63,792 & 86,119 & 1.35 & 34,752 & 35,816 & 1.03 \\
\hline Average & 17.15 & 15.63 & 168,927 & 132,497 & 37,731 & 49,189 & 1.16 & 31,092 & 43,284 & 1.37 & 33,917 & 42,826 & 1.24 & 32,366 & 36,922 & 1.14 \\
\hline p-value & .10 & -- & 0 & 0 & -- & -- & -- & -- & -- & -- & -- & -- & -- & -- & -- & -- \\
\hline slope & -.36 & -- & $-6,778$ & $-2,172$ & -- & -- & -- & -- & -- & -- & -- & -- & -- & -- & -- & -- \\
\hline \multicolumn{17}{|c|}{ Gray County (estimated mean soil permeability $=2.0$ inches per hour) } \\
\hline 1991 & 20.96 & 17.64 & 318,248 & 197,652 & 65,390 & 115,740 & 1.77 & 118,959 & 183,197 & 1.54 & NA & NA & NA & 13,303 & 19,311 & 1.45 \\
\hline 1992 & 27.71 & 21.25 & 234,663 & 192,815 & 62,571 & 82,594 & 1.32 & 104,756 & 122,565 & 1.17 & 12,741 & 15,799 & 1.24 & 12,747 & 13,706 & 1.08 \\
\hline 1993 & 27.37 & 21.75 & 210,610 & 187,749 & 54,146 & 67,141 & 1.24 & 116,515 & 128,167 & 1.10 & 4,963 & 3,921 & .79 & 12,125 & 11,382 & .94 \\
\hline 1994 & 17.32 & 15.18 & 255,990 & 189,857 & 48,740 & 74,572 & 1.53 & 112,570 & 147,467 & 1.31 & 11,809 & 15,116 & 1.28 & 16,738 & 18,835 & 1.13 \\
\hline 1995 & 19.81 & 18.50 & 249,139 & 191,401 & 41,995 & 66,352 & 1.58 & 117,669 & 143,556 & 1.22 & 15,315 & 19,297 & 1.26 & 16,422 & 19,934 & 1.21 \\
\hline 1996 & 30.99 & 29.49 & 217,497 & 188,268 & 33,518 & 46,255 & 1.38 & 123,790 & 136,169 & 1.10 & 12,301 & 14,269 & 1.16 & 18,659 & 20,804 & 1.11 \\
\hline 1997 & 22.85 & 20.12 & 223,707 & 193,405 & 20,748 & 28,010 & 1.35 & 86,094 & 94,703 & 1.10 & 62,101 & 75,142 & 1.21 & 24,462 & 25,852 & 1.06 \\
\hline 1998 & 18.15 & 15.35 & 254,503 & 194,869 & 16,422 & 26,275 & 1.60 & 76,398 & 97,789 & 1.28 & 76,388 & 100,068 & 1.31 & 25,661 & 30,370 & 1.18 \\
\hline 1999 & 23.29 & 22.06 & 207,754 & 196,852 & 13,436 & 17,198 & 1.28 & 70,347 & 70,347 & 1.00 & 86,700 & 93,636 & 1.08 & 26,369 & 26,573 & 1.01 \\
\hline 2000 & 17.48 & 16.46 & 262,448 & 196,639 & 11,805 & 18,180 & 1.54 & 54,826 & 66,888 & 1.22 & 104,458 & 141,018 & 1.35 & 25,550 & 36,362 & 1.42 \\
\hline 2001 & 17.05 & 14.87 & 242,452 & 193,058 & 9,062 & 12,868 & 1.42 & 58,037 & 66,743 & 1.15 & 103,222 & 132,124 & 1.28 & 22,737 & 30,717 & 1.35 \\
\hline 2002 & 12.85 & 11.64 & 268,045 & 184,726 & 7,280 & 13,031 & 1.79 & 50,502 & 66,158 & 1.31 & 102,352 & 150,457 & 1.47 & 24,592 & 38,399 & 1.56 \\
\hline 2003 & 19.73 & 17.98 & 233,741 & 181,426 & 5,429 & 7,221 & 1.33 & 33,938 & 46,156 & 1.36 & 121,067 & 153,755 & 1.27 & 20,992 & 26,610 & 1.27 \\
\hline Average & 21.20 & 18.64 & 244,523 & 191,440 & 30,042 & 44,264 & 1.47 & 86,492 & 105,377 & 1.22 & 59,451 & 76,217 & 1.23 & 20,027 & 24,527 & 1.22 \\
\hline $\mathrm{p}$-value & .06 & -- & 1.0 & .58 & -- & -- & -- & -- & -- & -- & -- & -- & -- & -- & -- & -- \\
\hline slope & -.60 & -- & 390 & -367 & -- & -- & -- & -- & -- & -- & -- & -- & -- & -- & -- & -- \\
\hline \multicolumn{17}{|c|}{ Greeley County (estimated mean soil permeability = 1.2 inches per hour) } \\
\hline 1991 & 20.55 & 17.42 & 39,803 & 27,211 & 18,519 & 27,779 & 1.50 & 6,950 & 10,008 & 1.44 & NA & NA & NA & 1,742 & 2,016 & 1.16 \\
\hline 1992 & 19.82 & 16.23 & 34,118 & 28,347 & 14,819 & 19,265 & 1.30 & 6,245 & 5,308 & .85 & 5,264 & 7,264 & 1.38 & 2,019 & 2,281 & 1.13 \\
\hline 1993 & 20.67 & 16.74 & 28,895 & 26,874 & 14,138 & 15,552 & 1.10 & 5,227 & 4,809 & .92 & 5,686 & 6,994 & 1.23 & 1,823 & 1,541 & .85 \\
\hline 1994 & 18.76 & 17.04 & 34,724 & 27,044 & 13,187 & 17,143 & 1.30 & 7,325 & 9,889 & 1.35 & 3,189 & 3,572 & 1.12 & 3,343 & 4,121 & 1.23 \\
\hline 1995 & 18.64 & 17.35 & 28,223 & 28,029 & 10,372 & 11,513 & 1.11 & 4,971 & 5,468 & 1.10 & 10,651 & 9,692 & .91 & 2,035 & 1,549 & .76 \\
\hline 1996 & 21.88 & 21.10 & 32,404 & 29,743 & 8,163 & 10,122 & 1.24 & 7,167 & 7,310 & 1.02 & 12,336 & 13,323 & 1.08 & 2,077 & 1,649 & .79 \\
\hline
\end{tabular}


Table 1. Precipitation, irrigation water use, acres irrigated, irrigation application rate by system type, and soil permeability for counties in the Groundwater Management Districts within the Kansas High Plains, 1991-2003.-Continued

[Precipitation data from Kansas State Climatologist (2005). Irrigation data from Kansas Water Office and Kansas Department of Agriculture (1993-2005). Soil permeability from U.S. Department of Agriculture (1994). Blue shading indicates trend is significant at 95-percent confidence level ( $\mathrm{p} \leq 0.05)$. Green shading indicates trend is significant at 90 -percent confidence level ( $\mathrm{p} \leq 0.10)$. acre-ft, acre-feet; acreft/acre, acre-feet per acre; NA, data not available; --, not computed]

\begin{tabular}{|c|c|c|c|c|c|c|c|c|c|c|c|c|c|c|c|c|}
\hline \multirow[b]{2}{*}{ Year } & \multirow[b]{2}{*}{$\begin{array}{l}\text { Annual } \\
\text { precip- } \\
\text { itation } \\
\text { (inches) }\end{array}$} & \multirow{2}{*}{$\begin{array}{l}\text { March- } \\
\text { October } \\
\text { precipi- } \\
\text { tation } \\
\text { (inches) }\end{array}$} & \multirow[b]{2}{*}{$\begin{array}{c}\text { Total } \\
\text { irrigation } \\
\text { water use } \\
\text { (acre-ft) }\end{array}$} & \multirow[b]{2}{*}{$\begin{array}{l}\text { Total acres } \\
\text { irrigated }\end{array}$} & \multicolumn{3}{|c|}{ Flood irrigation } & \multicolumn{3}{|c|}{ Center pivot irrigation } & \multicolumn{3}{|c|}{$\begin{array}{l}\text { Center pivot irrigation with } \\
\text { drop nozzle }\end{array}$} & \multicolumn{3}{|c|}{ Other irrigation systems } \\
\hline & & & & & $\begin{array}{c}\text { Total } \\
\text { acres } \\
\text { irrigated }\end{array}$ & $\begin{array}{c}\text { Water } \\
\text { used } \\
\text { (acre-ft) }\end{array}$ & $\begin{array}{l}\text { Rate of } \\
\text { irri- } \\
\text { gation } \\
\text { (acre- } \\
\text { ft/acre) }\end{array}$ & $\begin{array}{c}\text { Total } \\
\text { acres } \\
\text { irrigated }\end{array}$ & $\begin{array}{c}\text { Water } \\
\text { used } \\
\text { (acre-ft) }\end{array}$ & $\begin{array}{l}\text { Rate of } \\
\text { irri- } \\
\text { gation } \\
\text { (acre- } \\
\text { ft/acre) }\end{array}$ & $\begin{array}{c}\text { Total } \\
\text { acres } \\
\text { irrigated }\end{array}$ & $\begin{array}{c}\text { Water } \\
\text { used } \\
\text { (acre-ft) }\end{array}$ & $\begin{array}{l}\text { Rate of } \\
\text { irri- } \\
\text { gation } \\
\text { (acre- } \\
\text { ft/acre) }\end{array}$ & $\begin{array}{c}\text { Total } \\
\text { acres } \\
\text { irrigated }\end{array}$ & $\begin{array}{c}\text { Water } \\
\text { used } \\
\text { (acre-ft) }\end{array}$ & $\begin{array}{l}\text { Rate of } \\
\text { irri- } \\
\text { gation } \\
\text { (acre- } \\
\text { ft/acre) }\end{array}$ \\
\hline \multicolumn{17}{|c|}{ Greeley County (estimated mean soil permeability $=1.2$ inches per hour) —Continued } \\
\hline 1997 & 24.72 & 21.91 & 28,153 & 28,997 & 7,367 & 7,809 & 1.06 & 3,697 & 3,660 & 0.99 & 15,912 & 14,639 & 0.92 & 2,021 & 2,045 & 1.01 \\
\hline 1998 & 17.49 & 15.20 & 27,964 & 30,367 & 5,755 & 5,813 & 1.01 & 4,571 & 4,525 & .99 & 18,248 & 15,876 & .87 & 1,793 & 1,750 & .98 \\
\hline 1999 & 21.38 & 20.24 & 26,227 & 29,740 & 5,299 & 5,140 & .97 & 3,694 & 3,288 & .89 & 17,439 & 15,172 & .87 & 3,308 & 2,627 & .79 \\
\hline 2000 & 14.67 & 13.46 & 31,874 & 29,451 & 3,120 & 3,557 & 1.14 & 3,859 & 3,782 & .98 & 19,758 & 20,943 & 1.06 & 2,714 & 3,592 & 1.32 \\
\hline 2001 & 13.07 & 10.98 & 28,056 & 28,464 & 2,056 & 2,611 & 1.27 & 3,475 & 3,614 & 1.04 & 20,786 & 19,955 & .96 & 2,147 & 1,876 & .87 \\
\hline 2002 & 10.21 & 9.62 & 33,752 & 29,261 & 2,377 & 2,971 & 1.25 & 3,304 & 3,833 & 1.16 & 21,563 & 23,935 & 1.11 & 2,017 & 3,013 & 1.49 \\
\hline 2003 & 16.70 & 15.83 & 28,574 & 28,438 & 2,012 & 2,072 & 1.03 & 2,350 & 2,609 & 1.11 & 21,347 & 20,493 & .96 & 2,729 & 3,400 & 1.25 \\
\hline Average & 18.35 & 16.39 & 30,982 & 28,613 & 8,245 & 10,104 & 1.18 & 4,833 & 5,239 & 1.06 & 14,348 & 14,321 & 1.04 & 2,290 & 2,420 & 1.06 \\
\hline p-value & .04 & -- & .08 & .16 & -- & -- & -- & -- & -- & -- & -- & -- & -- & -- & -- & -- \\
\hline slope & -.64 & -- & -436 & 156 & & & & & & & & & & & & \\
\hline \multicolumn{17}{|c|}{ Harvey County (estimated mean soil permeability = 1.2 inches per hour) } \\
\hline 1991 & 24.96 & 20.20 & 32,641 & 27,337 & 11,421 & 15,190 & 1.33 & 13,860 & 15,662 & 1.13 & NA & NA & NA & 2,056 & 1,789 & .87 \\
\hline 1992 & 34.89 & 24.94 & 18,593 & 26,211 & 10,302 & 8,139 & .79 & 14,416 & 9,515 & .66 & 133 & 64 & .48 & 1,360 & 876 & .64 \\
\hline 1993 & 41.21 & 35.35 & 16,865 & 25,369 & 9,360 & 6,739 & .72 & 14,524 & 9,586 & .66 & 97 & 60 & .62 & 1,388 & 480 & .35 \\
\hline 1994 & 25.42 & 20.85 & 29,135 & 26,878 & 9,941 & 11,730 & 1.18 & 15,278 & 16,042 & 1.05 & 79 & 27 & .34 & 1,580 & 1,336 & .85 \\
\hline 1995 & 36.75 & 35.03 & 24,695 & 27,701 & 9,106 & 9,015 & .99 & 16,288 & 14,008 & .86 & 277 & 177 & .64 & 2,030 & 1,496 & .74 \\
\hline 1996 & 32.07 & 27.79 & 24,981 & 28,860 & 9,167 & 8,892 & .97 & 17,345 & 14,570 & .84 & 165 & 101 & .61 & 2,183 & 1,418 & .65 \\
\hline 1997 & 33.20 & 27.05 & 21,350 & 29,390 & 8,309 & 6,564 & .79 & 13,498 & 10,124 & .75 & 5,359 & 3,376 & .63 & 2,224 & 1,286 & .58 \\
\hline 1998 & 39.72 & 27.71 & 27,425 & 30,126 & 8,354 & 8,438 & 1.01 & 10,623 & 8,817 & .83 & 8,254 & 8,006 & .97 & 2,895 & 2,164 & .75 \\
\hline 1999 & 36.76 & 30.70 & 23,261 & 30,000 & 7,432 & 6,689 & .90 & 9,320 & 6,244 & .67 & 10,810 & 8,756 & .81 & 2,438 & 1,572 & .64 \\
\hline 2000 & 29.09 & 25.00 & 30,603 & 31,432 & 8,274 & 9,019 & 1.09 & 4,823 & 4,341 & .90 & 14,126 & 13,561 & .96 & 4,209 & 3,683 & .87 \\
\hline 2001 & 29.09 & 23.98 & 35,737 & 31,181 & 7,812 & 9,531 & 1.22 & 5,722 & 6,294 & 1.10 & 14,848 & 16,778 & 1.13 & 2,799 & 3,134 & 1.12 \\
\hline 2002 & 28.61 & 26.03 & 33,307 & 31,844 & 7,852 & 9,658 & 1.23 & 3,774 & 3,472 & .92 & 17,267 & 17,440 & 1.01 & 2,951 & 2,737 & .93 \\
\hline 2003 & 36.05 & 33.02 & 37,459 & 32,962 & 7,119 & 9,326 & 1.31 & 3,514 & 3,444 & .98 & 18,098 & 20,089 & 1.11 & 4,231 & 4,601 & 1.09 \\
\hline Average & 32.91 & 27.51 & 27,389 & 29,176 & 8,804 & 9,148 & 1.04 & 10,999 & 9,394 & .87 & 7,459 & 7,370 & .78 & 2,488 & 2,044 & .82 \\
\hline p-value & 1.0 & -- & .03 & 0 & -- & -- & -- & -- & -- & -- & -- & -- & -- & -- & -- & -- \\
\hline slope & -.04 & -- & 1,291 & 614 & -- & -- & -- & -- & -- & -- & -- & -- & -- & -- & -- & -- \\
\hline
\end{tabular}


Table 1. Precipitation, irrigation water use, acres irrigated, irrigation application rate by system type, and soil permeability for counties in the Groundwater Management Districts within the Kansas High Plains, 1991-2003.-Continued

[Precipitation data from Kansas State Climatologist (2005). Irrigation data from Kansas Water Office and Kansas Department of Agriculture (1993-2005). Soil permeability from U.S. Department of Agriculture (1994). Blue shading indicates trend is significant at 95 -percent confidence level ( $\mathrm{p} \leq 0.05$ ). Green shading indicates trend is significant at 90 -percent confidence level ( $\mathrm{p} \leq 0.10)$. acre-ft, acre-feet; acreft/acre, acre-feet per acre; NA, data not available; --, not computed]

\begin{tabular}{|c|c|c|c|c|c|c|c|c|c|c|c|c|c|c|c|c|}
\hline \multirow[b]{2}{*}{ Year } & \multirow[b]{2}{*}{$\begin{array}{l}\text { Annual } \\
\text { precip- } \\
\text { itation } \\
\text { (inches) }\end{array}$} & \multirow{2}{*}{$\begin{array}{l}\text { March- } \\
\text { October } \\
\text { precipi- } \\
\text { tation } \\
\text { (inches) }\end{array}$} & \multirow[b]{2}{*}{$\begin{array}{c}\text { Total } \\
\text { irrigation } \\
\text { water use } \\
\text { (acre-ft) }\end{array}$} & \multirow[b]{2}{*}{$\begin{array}{c}\text { Total acres } \\
\text { irrigated }\end{array}$} & \multicolumn{3}{|c|}{ Flood irrigation } & \multicolumn{3}{|c|}{ Center pivot irrigation } & \multicolumn{3}{|c|}{$\begin{array}{l}\text { Center pivot irrigation with } \\
\text { drop nozzle }\end{array}$} & \multicolumn{3}{|c|}{ Other irrigation systems } \\
\hline & & & & & $\begin{array}{c}\text { Total } \\
\text { acres } \\
\text { irrigated }\end{array}$ & $\begin{array}{c}\text { Water } \\
\text { used } \\
\text { (acre-ft) }\end{array}$ & $\begin{array}{l}\text { Rate of } \\
\text { irri- } \\
\text { gation } \\
\text { (acre- } \\
\text { ft/acre) }\end{array}$ & $\begin{array}{c}\text { Total } \\
\text { acres } \\
\text { irrigated }\end{array}$ & $\begin{array}{c}\text { Water } \\
\text { used } \\
\text { (acre-ft) }\end{array}$ & $\begin{array}{l}\text { Rate of } \\
\text { irri- } \\
\text { gation } \\
\text { (acre- } \\
\text { ft/acre) }\end{array}$ & $\begin{array}{c}\text { Total } \\
\text { acres } \\
\text { irrigated }\end{array}$ & $\begin{array}{c}\text { Water } \\
\text { used } \\
\text { (acre-ft) }\end{array}$ & $\begin{array}{l}\text { Rate of } \\
\text { irri- } \\
\text { gation } \\
\text { (acre- } \\
\text { ft/acre) }\end{array}$ & $\begin{array}{c}\text { Total } \\
\text { acres } \\
\text { irrigated }\end{array}$ & $\begin{array}{c}\text { Water } \\
\text { used } \\
\text { (acre-ft) }\end{array}$ & $\begin{array}{c}\text { Rate of } \\
\text { irri- } \\
\text { gation } \\
\text { (acre- } \\
\text { ft/acre) }\end{array}$ \\
\hline \multicolumn{17}{|c|}{ Haskell County (estimated mean soil permeability $=2.0$ inches per hour) } \\
\hline 1991 & 16.18 & 13.32 & 390,649 & 218,666 & 173,326 & 306,787 & 1.77 & 27,151 & 45,614 & 1.68 & NA & NA & NA & 18,189 & 38,248 & 2.10 \\
\hline 1992 & 16.77 & 12.91 & 331,144 & 217,245 & 162,954 & 257,467 & 1.58 & 30,958 & 43,651 & 1.41 & 3,282 & 2,954 & .90 & 20,051 & 27,072 & 1.35 \\
\hline 1993 & 22.20 & 18.05 & 280,433 & 216,578 & 150,017 & 204,023 & 1.36 & 37,417 & 45,275 & 1.21 & 3,649 & 3,977 & 1.09 & 25,495 & 27,158 & 1.07 \\
\hline 1994 & 16.28 & 14.87 & 338,265 & 218,088 & 130,875 & 215,944 & 1.65 & 39,261 & 55,358 & 1.41 & 4,621 & 7,255 & 1.57 & 43,331 & 59,708 & 1.38 \\
\hline 1995 & 14.73 & 13.80 & 322,206 & 213,309 & 98,663 & 160,821 & 1.63 & 53,293 & 74,077 & 1.39 & 3,383 & 3,451 & 1.02 & 57,970 & 83,857 & 1.45 \\
\hline 1996 & 23.93 & 22.40 & 283,368 & 212,300 & 79,310 & 122,931 & 1.55 & 61,830 & 72,341 & 1.17 & 2,978 & 2,561 & .86 & 68,182 & 85,536 & 1.25 \\
\hline 1997 & 21.71 & 19.58 & 266,590 & 213,416 & 68,622 & 98,129 & 1.43 & 40,655 & 51,225 & 1.26 & 42,412 & 50,470 & 1.19 & 61,727 & 66,765 & 1.08 \\
\hline 1998 & 18.74 & 16.04 & 259,642 & 214,409 & 57,196 & 77,215 & 1.35 & 41,452 & 50,986 & 1.23 & 47,060 & 57,413 & 1.22 & 68,701 & 74,028 & 1.08 \\
\hline 1999 & 23.81 & 22.25 & 230,834 & 212,874 & 46,298 & 60,187 & 1.30 & 40,815 & 43,672 & 1.07 & 46,806 & 51,019 & 1.09 & 78,955 & 75,956 & .96 \\
\hline 2000 & 17.28 & 16.42 & 275,682 & 210,115 & 39,840 & 58,963 & 1.48 & 29,624 & 38,807 & 1.31 & 66,585 & 87,892 & 1.32 & 74,066 & 90,019 & 1.22 \\
\hline 2001 & 12.63 & 10.37 & 240,724 & 200,480 & 31,059 & 43,483 & 1.40 & 23,428 & 26,942 & 1.15 & 77,448 & 94,487 & 1.22 & 68,545 & 75,813 & 1.11 \\
\hline 2002 & 11.86 & 10.72 & 303,666 & 213,401 & 25,285 & 47,030 & 1.86 & 25,650 & 34,371 & 1.34 & 76,074 & 103,461 & 1.36 & 86,392 & 118,804 & 1.38 \\
\hline 2003 & 13.98 & 13.14 & 249,435 & 199,953 & 18,106 & 28,245 & 1.56 & 20,536 & 22,590 & 1.10 & 76,927 & 96,928 & 1.26 & 84,384 & 101,672 & 1.20 \\
\hline Average & 17.70 & 15.68 & 290,203 & 212,372 & 83,196 & 129,325 & 1.53 & 36,313 & 46,531 & 1.29 & 37,602 & 46,822 & 1.18 & 58,153 & 71,126 & 1.22 \\
\hline p-value & .30 & -- & .01 & 0 & -- & -- & -- & -- & -- & -- & -- & -- & -- & -- & -- & -- \\
\hline slope & -.36 & -- & $-8,922$ & -979 & -- & -- & -- & -- & -- & -- & -- & -- & -- & -- & -- & -- \\
\hline \multicolumn{17}{|c|}{ Kearny County (estimated mean soil permeability $=2.4$ inches per hour) } \\
\hline 1991 & 18.76 & 15.75 & 168,070 & 98,578 & 46,072 & 65,883 & 1.43 & 48,078 & 91,829 & 1.91 & NA & NA & NA & 4,428 & 10,358 & 2.34 \\
\hline 1992 & 18.62 & 15.54 & 133,546 & 97,766 & 46,238 & 45,776 & .99 & 47,605 & 82,357 & 1.73 & 629 & 1,107 & 1.76 & 3,294 & 4,306 & 1.31 \\
\hline 1993 & 21.65 & 18.33 & 125,288 & 98,361 & 42,890 & 37,743 & .88 & 49,430 & 80,571 & 1.63 & 1,555 & 2,208 & 1.42 & 4,486 & 4,766 & 1.06 \\
\hline 1994 & 24.22 & 22.10 & 149,561 & 97,871 & 35,785 & 42,942 & 1.20 & 49,623 & 88,825 & 1.79 & 3,394 & 4,718 & 1.39 & 9,069 & 13,076 & 1.44 \\
\hline 1995 & 20.98 & 20.42 & 129,483 & 96,063 & 30,170 & 27,455 & .91 & 46,772 & 78,577 & 1.68 & 6,656 & 8,253 & 1.24 & 12,465 & 15,198 & 1.22 \\
\hline 1996 & 23.20 & 22.29 & 131,765 & 97,004 & 28,028 & 27,187 & .97 & 52,148 & 84,480 & 1.62 & 6,699 & 9,379 & 1.40 & 10,129 & 10,719 & 1.06 \\
\hline 1997 & 24.42 & 22.38 & 131,882 & 104,172 & 25,776 & 20,621 & .80 & 38,677 & 61,883 & 1.60 & 25,973 & 37,141 & 1.43 & 13,746 & 12,236 & .89 \\
\hline 1998 & 20.96 & 17.67 & 131,906 & 103,819 & 22,644 & 16,530 & .73 & 42,474 & 67,534 & 1.59 & 23,093 & 33,947 & 1.47 & 15,608 & 13,895 & .89 \\
\hline 1999 & 16.75 & 15.35 & 133,931 & 103,050 & 20,706 & 14,908 & .72 & 35,116 & 53,025 & 1.51 & 32,607 & 50,215 & 1.54 & 14,621 & 15,782 & 1.08 \\
\hline 2000 & 17.01 & 15.94 & 161,529 & 103,217 & 18,780 & 16,526 & .88 & 15,867 & 30,147 & 1.90 & 56,383 & 101,489 & 1.80 & 12,187 & 13,365 & 1.10 \\
\hline 2001 & 18.86 & 16.84 & 132,076 & 101,111 & 18,118 & 11,958 & .66 & 12,852 & 19,792 & 1.54 & 54,961 & 85,739 & 1.56 & 15,180 & 14,587 & .96 \\
\hline 2002 & 10.51 & 9.43 & 185,629 & 107,275 & 17,534 & 23,846 & 1.36 & 12,711 & 25,803 & 2.03 & 59,970 & 112,144 & 1.87 & 17,060 & 23,835 & 1.40 \\
\hline
\end{tabular}


Table 1. Precipitation, irrigation water use, acres irrigated, irrigation application rate by system type, and soil permeability for counties in the Groundwater Management Districts within the Kansas High Plains, 1991-2003.-Continued

[Precipitation data from Kansas State Climatologist (2005). Irrigation data from Kansas Water Office and Kansas Department of Agriculture (1993-2005). Soil permeability from U.S. Department of Agriculture (1994). Blue shading indicates trend is significant at 95-percent confidence level ( $\mathrm{p} \leq 0.05)$. Green shading indicates trend is significant at 90-percent confidence level ( $\mathrm{p} \leq 0.10)$. acre-ft, acre-feet; acreft/acre, acre-feet per acre; NA, data not available; --, not computed]

\begin{tabular}{|c|c|c|c|c|c|c|c|c|c|c|c|c|c|c|c|c|}
\hline \multirow[b]{2}{*}{ Year } & \multirow[b]{2}{*}{$\begin{array}{l}\text { Annual } \\
\text { precip- } \\
\text { itation } \\
\text { (inches) }\end{array}$} & \multirow{2}{*}{$\begin{array}{l}\text { March- } \\
\text { October } \\
\text { precipi- } \\
\text { tation } \\
\text { (inches) }\end{array}$} & \multirow[b]{2}{*}{$\begin{array}{c}\text { Total } \\
\text { irrigation } \\
\text { water use } \\
\text { (acre-ft) }\end{array}$} & \multirow[b]{2}{*}{$\begin{array}{c}\text { Total acres } \\
\text { irrigated }\end{array}$} & \multicolumn{3}{|c|}{ Flood irrigation } & \multicolumn{3}{|c|}{ Center pivot irrigation } & \multicolumn{3}{|c|}{$\begin{array}{l}\text { Center pivot irrigation with } \\
\text { drop nozzle }\end{array}$} & \multicolumn{3}{|c|}{ Other irrigation systems } \\
\hline & & & & & $\begin{array}{c}\text { Total } \\
\text { acres } \\
\text { irrigated }\end{array}$ & $\begin{array}{c}\text { Water } \\
\text { used } \\
\text { (acre-ft) }\end{array}$ & $\begin{array}{l}\text { Rate of } \\
\text { irri- } \\
\text { gation } \\
\text { (acre- } \\
\text { ft/acre) }\end{array}$ & $\begin{array}{c}\text { Total } \\
\text { acres } \\
\text { irrigated }\end{array}$ & $\begin{array}{c}\text { Water } \\
\text { used } \\
\text { (acre-ft) }\end{array}$ & $\begin{array}{l}\text { Rate of } \\
\text { irri- } \\
\text { gation } \\
\text { (acre- } \\
\text { ft/acre) }\end{array}$ & $\begin{array}{c}\text { Total } \\
\text { acres } \\
\text { irrigated }\end{array}$ & $\begin{array}{c}\text { Water } \\
\text { used } \\
\text { (acre-ft) }\end{array}$ & $\begin{array}{c}\text { Rate of } \\
\text { irri- } \\
\text { gation } \\
\text { (acre- } \\
\text { ft/acre) }\end{array}$ & $\begin{array}{c}\text { Total } \\
\text { acres } \\
\text { irrigated }\end{array}$ & $\begin{array}{c}\text { Water } \\
\text { used } \\
\text { (acre-ft) }\end{array}$ & $\begin{array}{l}\text { Rate of } \\
\text { irri- } \\
\text { gation } \\
\text { (acre- } \\
\mathrm{ft} / \text { acre) }\end{array}$ \\
\hline \multicolumn{17}{|c|}{ Kearny County (estimated mean soil permeability $=2.4$ inches per hour) - Continued } \\
\hline 2003 & 18.06 & 16.36 & 146,641 & 103,846 & 14,169 & 13,886 & 0.98 & 11,731 & 19,122 & 1.63 & 59,041 & 91,514 & 1.55 & 18,905 & 22,120 & 1.17 \\
\hline Average & 19.54 & 17.57 & 143,177 & 100,933 & 28,224 & 28,097 & .96 & 35,622 & 60,303 & 1.70 & 27,580 & 44,821 & 1.54 & 11,629 & 13,403 & 1.15 \\
\hline p-value & .16 & -- & .20 & .06 & -- & -- & -- & -- & -- & -- & -- & -- & -- & -- & -- & -- \\
\hline slope & -.36 & -- & 936 & 642 & -- & -- & -- & -- & -- & -- & -- & -- & -- & -- & -- & -- \\
\hline \multicolumn{17}{|c|}{ Kiowa County (estimated mean soil permeability $=3.7$ inches per hour) } \\
\hline 1991 & 18.77 & 15.65 & 75,245 & 49,214 & 1,426 & 2,467 & 1.73 & 46,826 & 71,644 & 1.53 & NA & NA & NA & 962 & 1,134 & 1.18 \\
\hline 1992 & 26.89 & 22.47 & 47,130 & 48,883 & 936 & 936 & 1.00 & 46,425 & 44,568 & .96 & 1,075 & 1,118 & 1.04 & 447 & 508 & 1.14 \\
\hline 1993 & 31.50 & 26.32 & 51,802 & 48,559 & 930 & 1,163 & 1.25 & 45,279 & 47,543 & 1.05 & 1,546 & 2,180 & 1.41 & 804 & 917 & 1.14 \\
\hline 1994 & 21.60 & 18.17 & 66,869 & 48,812 & 1,044 & 1,608 & 1.54 & 45,630 & 62,969 & 1.38 & 1,777 & 2,044 & 1.15 & 361 & 249 & .69 \\
\hline 1995 & 24.59 & 23.28 & 55,885 & 48,684 & 868 & 1,215 & 1.40 & 45,470 & 52,291 & 1.15 & 1,714 & 1,937 & 1.13 & 632 & 442 & .70 \\
\hline 1996 & 31.44 & 29.14 & 45,897 & 50,672 & 830 & 838 & 1.01 & 46,147 & 41,994 & .91 & 3,022 & 2,508 & .83 & 673 & 557 & .83 \\
\hline 1997 & 31.01 & 26.89 & 44,379 & 51,365 & 585 & 649 & 1.11 & 32,328 & 27,479 & .85 & 18,164 & 16,166 & .89 & 288 & 85 & .29 \\
\hline 1998 & 22.41 & 18.17 & 69,232 & 51,852 & 604 & 918 & 1.52 & 25,207 & 33,525 & 1.33 & 23,721 & 32,023 & 1.35 & 2,320 & 2,765 & 1.19 \\
\hline 1999 & 21.66 & 20.55 & 59,727 & 52,023 & 409 & 519 & 1.27 & 23,074 & 26,535 & 1.15 & 25,932 & 30,081 & 1.16 & 2,608 & 2,591 & .99 \\
\hline 2000 & 33.72 & 31.02 & 64,090 & 51,852 & 261 & 326 & 1.25 & 16,010 & 20,013 & 1.25 & 33,672 & 42,090 & 1.25 & 1,909 & 1,661 & .87 \\
\hline 2001 & 24.16 & 17.81 & 70,605 & 51,539 & 413 & 297 & .72 & 15,593 & 22,454 & 1.44 & 33,846 & 45,692 & 1.35 & 1,687 & 2,161 & 1.28 \\
\hline 2002 & 22.33 & 20.85 & 69,065 & 52,851 & 431 & 491 & 1.14 & 12,353 & 17,788 & 1.44 & 37,464 & 47,205 & 1.26 & 2,603 & 3,581 & 1.38 \\
\hline 2003 & 19.00 & 17.31 & 67,440 & 52,629 & 385 & 485 & 1.26 & 10,304 & 14,838 & 1.44 & 39,894 & 49,070 & 1.23 & 2,046 & 3,047 & 1.49 \\
\hline Average & 25.31 & 22.13 & 60,567 & 50,687 & 702 & 916 & 1.25 & 31,588 & 37,203 & 1.22 & 18,486 & 22,676 & 1.17 & 1,334 & 1,515 & 1.14 \\
\hline p-value & .67 & -- & .36 & 0 & -- & -- & -- & -- & -- & -- & -- & -- & -- & -- & -- & -- \\
\hline slope & -.20 & -- & 1,382 & 360 & -- & -- & -- & -- & -- & -- & -- & -- & -- & -- & -- & -- \\
\hline \multicolumn{17}{|c|}{ Lane County (estimated mean soil permeability $=1.2$ inches per hour) } \\
\hline 1991 & 21.56 & 18.74 & 27,668 & 24,427 & 21,865 & 26,019 & 1.19 & 1,707 & 1,349 & .79 & NA & NA & NA & 855 & 300 & .35 \\
\hline 1992 & 25.66 & 20.44 & 16,615 & 20,264 & 15,898 & 14,785 & .93 & 2,301 & 1,220 & .53 & 0 & 0 & 0 & 2,065 & 610 & .30 \\
\hline 1993 & 29.73 & 23.97 & 11,164 & 17,080 & 13,171 & 9,351 & .71 & 1,821 & 819 & .45 & 490 & 431 & .88 & 1,598 & 562 & .35 \\
\hline 1994 & 22.78 & 18.73 & 20,020 & 18,490 & 14,635 & 16,099 & 1.10 & 1,508 & 1,191 & .79 & 864 & 1,140 & 1.32 & 1,483 & 1,590 & 1.07 \\
\hline 1995 & 21.13 & 20.07 & 21,075 & 21,705 & 14,669 & 15,402 & 1.05 & 1,729 & 1,245 & .72 & 1,305 & 1,736 & 1.33 & 4,002 & 2,692 & .67 \\
\hline 1996 & 32.79 & 31.21 & 15,347 & 21,230 & 13,299 & 10,240 & .77 & 2,014 & 1,611 & .80 & 2,168 & 1,734 & .80 & 3,749 & 1,761 & .47 \\
\hline
\end{tabular}


Table 1. Precipitation, irrigation water use, acres irrigated, irrigation application rate by system type, and soil permeability for counties in the Groundwater Management Districts within the Kansas High Plains, 1991-2003.-Continued

[Precipitation data from Kansas State Climatologist (2005). Irrigation data from Kansas Water Office and Kansas Department of Agriculture (1993-2005). Soil permeability from U.S. Department of Agriculture (1994). Blue shading indicates trend is significant at 95 -percent confidence level ( $\mathrm{p} \leq 0.05$ ). Green shading indicates trend is significant at 90 -percent confidence level ( $\mathrm{p} \leq 0.10)$. acre-ft, acre-feet; acreft/acre, acre-feet per acre; NA, data not available; --, not computed]

\begin{tabular}{|c|c|c|c|c|c|c|c|c|c|c|c|c|c|c|c|c|}
\hline \multirow[b]{2}{*}{ Year } & \multirow[b]{2}{*}{$\begin{array}{l}\text { Annual } \\
\text { precip- } \\
\text { itation } \\
\text { (inches) }\end{array}$} & \multirow{2}{*}{$\begin{array}{l}\text { March- } \\
\text { October } \\
\text { precipi- } \\
\text { tation } \\
\text { (inches) }\end{array}$} & \multirow[b]{2}{*}{$\begin{array}{c}\text { Total } \\
\text { irrigation } \\
\text { water use } \\
\text { (acre-ft) }\end{array}$} & \multirow[b]{2}{*}{$\begin{array}{l}\text { Total acres } \\
\text { irrigated }\end{array}$} & \multicolumn{3}{|c|}{ Flood irrigation } & \multicolumn{3}{|c|}{ Center pivot irrigation } & \multicolumn{3}{|c|}{$\begin{array}{l}\text { Center pivot irrigation with } \\
\text { drop nozzle }\end{array}$} & \multicolumn{3}{|c|}{ Other irrigation systems } \\
\hline & & & & & $\begin{array}{c}\text { Total } \\
\text { acres } \\
\text { irrigated }\end{array}$ & $\begin{array}{c}\text { Water } \\
\text { used } \\
\text { (acre-ft) }\end{array}$ & $\begin{array}{l}\text { Rate of } \\
\text { irri- } \\
\text { gation } \\
\text { (acre- } \\
\text { ft/acre) }\end{array}$ & $\begin{array}{c}\text { Total } \\
\text { acres } \\
\text { irrigated }\end{array}$ & $\begin{array}{c}\text { Water } \\
\text { used } \\
\text { (acre-ft) }\end{array}$ & $\begin{array}{l}\text { Rate of } \\
\text { irri- } \\
\text { gation } \\
\text { (acre- } \\
\text { ft/acre) }\end{array}$ & $\begin{array}{c}\text { Total } \\
\text { acres } \\
\text { irrigated }\end{array}$ & $\begin{array}{c}\text { Water } \\
\text { used } \\
\text { (acre-ft) }\end{array}$ & $\begin{array}{l}\text { Rate of } \\
\text { irri- } \\
\text { gation } \\
\text { (acre- } \\
\text { ft/acre) }\end{array}$ & $\begin{array}{c}\text { Total } \\
\text { acres } \\
\text { irrigated }\end{array}$ & $\begin{array}{c}\text { Water } \\
\text { used } \\
\text { (acre-ft) }\end{array}$ & $\begin{array}{l}\text { Rate of } \\
\text { irri- } \\
\text { gation } \\
\text { (acre- } \\
\text { ft/acre) }\end{array}$ \\
\hline \multicolumn{17}{|c|}{ Lane County (estimated mean soil permeability $=1.2$ inches per hour)-Continued } \\
\hline 1997 & 24.81 & 21.29 & 14,951 & 21,445 & 12,731 & 9,548 & 0.75 & 1,876 & 1,219 & 0.65 & 3,044 & 1,918 & 0.63 & 3,794 & 2,265 & 0.60 \\
\hline 1998 & 24.43 & 19.91 & 15,083 & 22,295 & 10,479 & 8,069 & .77 & 2,075 & 1,432 & .69 & 4,030 & 2,539 & .63 & 5,711 & 3,044 & .53 \\
\hline 1999 & 18.48 & 17.58 & 16,707 & 23,162 & 9,890 & 8,901 & .90 & 1,379 & 1,269 & .92 & 4,982 & 3,089 & .62 & 6,911 & 3,448 & .50 \\
\hline 2000 & 19.92 & 17.90 & 18,864 & 22,912 & 8,026 & 7,464 & .93 & 484 & 581 & 1.20 & 7,206 & 4,900 & .68 & 7,196 & 5,919 & .82 \\
\hline 2001 & 19.53 & 17.57 & 16,858 & 19,507 & 6,403 & 6,403 & 1.00 & 593 & 433 & .73 & 6,358 & 4,578 & .72 & 6,153 & 5,445 & .88 \\
\hline 2002 & 13.66 & 12.50 & 18,488 & 18,965 & 4,921 & 5,856 & 1.19 & 1,330 & 1,796 & 1.35 & 5,765 & 4,554 & .79 & 6,949 & 6,282 & .90 \\
\hline 2003 & 19.45 & 18.20 & 20,973 & 20,137 & 7,225 & 8,598 & 1.19 & 255 & 467 & 1.83 & 7,083 & 6,304 & .89 & 5,574 & 5,605 & 1.01 \\
\hline Average & 22.61 & 19.85 & 17,986 & 20,894 & 11,786 & 11,287 & .96 & 1,467 & 1,125 & .88 & 3,608 & 2,744 & .77 & 4,311 & 3,040 & .71 \\
\hline p-value & .02 & -- & .67 & 1.0 & -- & -- & -- & -- & -- & -- & -- & -- & -- & -- & -- & -- \\
\hline slope & -.70 & -- & 119 & 24 & -- & -- & -- & -- & -- & -- & -- & -- & -- & -- & -- & -- \\
\hline \multicolumn{17}{|c|}{ McPherson County (estimated mean soil permeability $=0.9$ inches per hour) } \\
\hline 1991 & 24.36 & 20.11 & 36,866 & 30,435 & 16,609 & 22,256 & 1.34 & 12,077 & 12,802 & 1.06 & NA & NA & NA & 1,749 & 1,808 & 1.03 \\
\hline 1992 & 37.63 & 30.75 & 12,316 & 27,899 & 14,571 & 7,431 & .51 & 10,813 & 3,785 & .35 & 796 & 279 & .35 & 1,719 & 821 & .48 \\
\hline 1993 & 46.78 & 40.53 & 12,389 & 25,109 & 12,372 & 6,928 & .56 & 10,982 & 4,503 & .41 & 245 & 103 & .42 & 1,510 & 855 & .57 \\
\hline 1994 & 20.33 & 15.41 & 30,414 & 29,532 & 15,211 & 17,645 & 1.16 & 11,030 & 9,817 & .89 & 246 & 308 & 1.25 & 3,045 & 2,645 & .87 \\
\hline 1995 & 37.90 & 35.59 & 23,802 & 28,217 & 13,453 & 13,049 & .97 & 12,143 & 8,743 & .72 & 258 & 219 & .85 & 2,363 & 1,791 & .76 \\
\hline 1996 & 31.41 & 27.84 & 24,149 & 30,722 & 13,147 & 12,095 & .92 & 14,093 & 9,583 & .68 & 698 & 719 & 1.03 & 2,784 & 1,752 & .63 \\
\hline 1997 & 35.66 & 29.77 & 22,168 & 31,455 & 12,397 & 10,537 & .85 & 8,714 & 4,967 & .57 & 7,039 & 4,435 & .63 & 3,305 & 2,229 & .67 \\
\hline 1998 & 38.06 & 28.85 & 27,394 & 32,724 & 11,387 & 11,501 & 1.01 & 9,657 & 6,663 & .69 & 7,118 & 5,623 & .79 & 4,562 & 3,607 & .79 \\
\hline 1999 & 38.69 & 32.78 & 20,366 & 32,561 & 10,785 & 8,304 & .77 & 7,963 & 4,300 & .54 & 9,745 & 5,067 & .52 & 4,068 & 2,694 & .66 \\
\hline 2000 & 31.96 & 26.69 & 28,787 & 33,088 & 11,303 & 12,320 & 1.09 & 8,050 & 5,474 & .68 & 9,759 & 7,710 & .79 & 3,976 & 3,283 & .83 \\
\hline 2001 & 28.26 & 23.45 & 33,159 & 33,269 & 10,815 & 12,978 & 1.20 & 6,795 & 5,640 & .83 & 10,779 & 9,809 & .91 & 4,880 & 4,732 & .97 \\
\hline 2002 & 29.02 & 25.65 & 35,375 & 34,548 & 10,195 & 12,336 & 1.21 & 5,920 & 5,565 & .94 & 12,709 & 11,946 & .94 & 5,724 & 5,528 & .97 \\
\hline 2003 & 31.80 & 28.21 & 39,036 & 33,988 & 9,307 & 12,471 & 1.34 & 6,270 & 6,709 & 1.07 & 12,634 & 13,392 & 1.06 & 5,777 & 6,464 & 1.12 \\
\hline Average & 33.22 & 28.13 & 26,632 & 31,042 & 12,427 & 12,296 & .99 & 9,577 & 6,812 & .73 & 6,002 & 4,967 & .80 & 3,497 & 2,939 & .84 \\
\hline p-value & .85 & -- & .06 & 0 & -- & -- & -- & -- & -- & -- & -- & -- & -- & -- & -- & -- \\
\hline slope & -.40 & -- & 1,836 & 595 & -- & -- & -- & -- & -- & -- & -- & -- & -- & -- & -- & -- \\
\hline
\end{tabular}


Table 1. Precipitation, irrigation water use, acres irrigated, irrigation application rate by system type, and soil permeability for counties in the Groundwater Management Districts within the Kansas High Plains, 1991-2003.-Continued

[Precipitation data from Kansas State Climatologist (2005). Irrigation data from Kansas Water Office and Kansas Department of Agriculture (1993-2005). Soil permeability from U.S. Department of Agriculture (1994). Blue shading indicates trend is significant at 95-percent confidence level $(\mathrm{p}<0.05)$. Green shading indicates trend is significant at 90 -percent confidence level $(\mathrm{p}<0.10)$. acre-ft, acre-feet; acreft/acre, acre-feet per acre; NA, data not available; --, not computed]

\begin{tabular}{|c|c|c|c|c|c|c|c|c|c|c|c|c|c|c|c|c|}
\hline \multirow[b]{2}{*}{ Year } & \multirow[b]{2}{*}{$\begin{array}{l}\text { Annual } \\
\text { precip- } \\
\text { itation } \\
\text { (inches) }\end{array}$} & \multirow{2}{*}{$\begin{array}{l}\text { March- } \\
\text { October } \\
\text { precipi- } \\
\text { tation } \\
\text { (inches) }\end{array}$} & \multirow[b]{2}{*}{$\begin{array}{c}\text { Total } \\
\text { irrigation } \\
\text { water use } \\
\text { (acre-ft) }\end{array}$} & \multirow[b]{2}{*}{$\begin{array}{c}\text { Total acres } \\
\text { irrigated }\end{array}$} & \multicolumn{3}{|c|}{ Flood irrigation } & \multicolumn{3}{|c|}{ Center pivot irrigation } & \multicolumn{3}{|c|}{$\begin{array}{l}\text { Center pivot irrigation with } \\
\text { drop nozzle }\end{array}$} & \multicolumn{3}{|c|}{ Other irrigation systems } \\
\hline & & & & & $\begin{array}{c}\text { Total } \\
\text { acres } \\
\text { irrigated }\end{array}$ & $\begin{array}{c}\text { Water } \\
\text { used } \\
\text { (acre-ft) }\end{array}$ & $\begin{array}{c}\text { Rate of } \\
\text { irri- } \\
\text { gation } \\
\text { (acre- } \\
\text { ft/acre) }\end{array}$ & $\begin{array}{c}\text { Total } \\
\text { acres } \\
\text { irrigated }\end{array}$ & $\begin{array}{c}\text { Water } \\
\text { used } \\
\text { (acre-ft) }\end{array}$ & $\begin{array}{l}\text { Rate of } \\
\text { irri- } \\
\text { gation } \\
\text { (acre- } \\
\text { ft/acre) }\end{array}$ & $\begin{array}{c}\text { Total } \\
\text { acres } \\
\text { irrigated }\end{array}$ & $\begin{array}{c}\text { Water } \\
\text { used } \\
\text { (acre-ft) }\end{array}$ & $\begin{array}{c}\text { Rate of } \\
\text { irri- } \\
\text { gation } \\
\text { (acre- } \\
\text { ft/acre) }\end{array}$ & $\begin{array}{c}\text { Total } \\
\text { acres } \\
\text { irrigated }\end{array}$ & $\begin{array}{c}\text { Water } \\
\text { used } \\
\text { (acre-ft) }\end{array}$ & $\begin{array}{c}\text { Rate of } \\
\text { irri- } \\
\text { gation } \\
\text { (acre- } \\
\mathrm{ft} / \text { acre) }\end{array}$ \\
\hline \multicolumn{17}{|c|}{ Meade County (estimated mean soil permeability = 2.2 inches per hour) } \\
\hline 1991 & 19.14 & 14.88 & 216,129 & 124,784 & 84,112 & 160,654 & 1.91 & 23,287 & 31,670 & 1.36 & NA & NA & NA & 17,385 & 23,805 & 1.37 \\
\hline 1992 & 18.90 & 15.02 & 168,859 & 123,552 & 84,747 & 126,273 & 1.49 & 24,077 & 24,318 & 1.01 & 1,070 & 1,102 & 1.03 & 13,658 & 17,166 & 1.26 \\
\hline 1993 & 17.89 & 13.88 & 161,882 & 124,442 & 83,461 & 120,184 & 1.44 & 25,808 & 26,066 & 1.01 & 2,000 & 1,700 & .85 & 13,173 & 13,932 & 1.06 \\
\hline 1994 & 21.87 & 20.34 & 182,199 & 122,846 & 73,467 & 119,017 & 1.62 & 28,608 & 35,760 & 1.25 & 2,768 & 3,654 & 1.32 & 18,003 & 23,769 & 1.32 \\
\hline 1995 & 20.66 & 19.43 & 173,885 & 119,254 & 65,295 & 102,513 & 1.57 & 31,331 & 41,357 & 1.32 & 1,091 & 1,353 & 1.24 & 21,537 & 28,662 & 1.33 \\
\hline 1996 & 26.76 & 23.53 & 166,599 & 124,793 & 65,867 & 97,483 & 1.48 & 38,692 & 44,109 & 1.14 & 1,728 & 1,503 & .87 & 18,506 & 23,504 & 1.27 \\
\hline 1997 & 28.14 & 22.48 & 144,351 & 124,399 & 48,169 & 61,656 & 1.28 & 26,595 & 27,925 & 1.05 & 20,463 & 22,919 & 1.12 & 29,172 & 31,852 & 1.09 \\
\hline 1998 & 27.42 & 26.46 & 170,925 & 124,726 & 34,202 & 51,987 & 1.52 & 22,098 & 28,064 & 1.27 & 32,483 & 42,878 & 1.32 & 35,943 & 47,996 & 1.34 \\
\hline 1999 & 27.35 & 26.19 & 142,101 & 126,820 & 28,241 & 36,431 & 1.29 & 21,333 & 24,106 & 1.13 & 38,255 & 41,315 & 1.08 & 38,991 & 40,248 & 1.03 \\
\hline 2000 & 21.38 & 19.82 & 179,978 & 128,336 & 25,546 & 39,341 & 1.54 & 14,869 & 22,304 & 1.50 & 51,214 & 71,187 & 1.39 & 36,707 & 47,146 & 1.28 \\
\hline 2001 & 24.63 & 16.92 & 160,400 & 118,987 & 16,424 & 23,486 & 1.43 & 15,242 & 22,558 & 1.48 & 51,326 & 67,750 & 1.32 & 35,995 & 46,605 & 1.29 \\
\hline 2002 & 23.36 & 21.06 & 201,544 & 125,890 & 17,978 & 28,945 & 1.61 & 16,013 & 27,382 & 1.71 & 53,905 & 87,326 & 1.62 & 37,994 & 57,891 & 1.52 \\
\hline 2003 & 33.72 & 32.41 & 156,630 & 122,229 & 13,148 & 16,829 & 1.28 & 11,734 & 13,025 & 1.11 & 57,672 & 77,280 & 1.34 & 39,675 & 49,495 & 1.25 \\
\hline Average & 23.94 & 20.96 & 171,191 & 123,928 & 49,281 & 75,754 & 1.50 & 23,053 & 28,357 & 1.26 & 26,165 & 34,997 & 1.21 & 27,441 & 34,775 & 1.27 \\
\hline p-value & .03 & -- & .30 & .76 & -- & -- & -- & -- & -- & -- & -- & -- & -- & -- & -- & -- \\
\hline slope & .87 & -- & $-1,282$ & 108 & -- & -- & -- & & -- & -- & -- & -- & -- & -- & -- & -- \\
\hline \multicolumn{17}{|c|}{ Morton County (estimated mean soil permeability $=2.5$ inches per hour) } \\
\hline 1991 & 18.40 & 15.50 & 83,832 & 50,778 & 34,871 & 58,235 & 1.67 & 10,113 & 16,080 & 1.59 & NA & NA & NA & 5,794 & 9,518 & 1.64 \\
\hline 1992 & 14.92 & 12.70 & 74,013 & 49,648 & 31,931 & 47,897 & 1.50 & 7,448 & 10,278 & 1.38 & 2,112 & 2,577 & 1.22 & 8,157 & 13,261 & 1.63 \\
\hline 1993 & 19.65 & 16.43 & 67,257 & 53,025 & 28,607 & 36,903 & 1.29 & 12,580 & 16,228 & 1.29 & 2,163 & 2,877 & 1.33 & 9,675 & 11,249 & 1.16 \\
\hline 1994 & 18.51 & 16.85 & 65,899 & 52,123 & 24,102 & 30,128 & 1.25 & 12,262 & 16,799 & 1.37 & 2,835 & 2,920 & 1.03 & 12,924 & 16,052 & 1.24 \\
\hline 1995 & 15.74 & 14.41 & 60,528 & 49,624 & 18,184 & 22,730 & 1.25 & 14,203 & 16,902 & 1.19 & 3,755 & 4,243 & 1.13 & 13,482 & 16,654 & 1.24 \\
\hline 1996 & 21.78 & 20.79 & 56,036 & 52,208 & 19,814 & 19,616 & .99 & 13,032 & 14,726 & 1.13 & 5,213 & 6,256 & 1.20 & 14,149 & 15,438 & 1.09 \\
\hline 1997 & 19.30 & 17.10 & 53,473 & 50,965 & 13,122 & 11,941 & .91 & 13,205 & 14,790 & 1.12 & 14,112 & 15,100 & 1.07 & 10,526 & 11,642 & 1.11 \\
\hline 1998 & 20.92 & 18.63 & 54,533 & 49,924 & 8,182 & 7,527 & .92 & 11,243 & 12,929 & 1.15 & 19,675 & 21,446 & 1.09 & 10,824 & 12,630 & 1.17 \\
\hline 1999 & 18.14 & 16.87 & 48,298 & 52,460 & 8,755 & 7,267 & .83 & 11,255 & 11,368 & 1.01 & 24,146 & 21,973 & .91 & 8,304 & 7,691 & .93 \\
\hline 2000 & 16.54 & 16.01 & 55,442 & 50,547 & 6,666 & 6,799 & 1.02 & 11,228 & 13,698 & 1.22 & 26,148 & 29,286 & 1.12 & 6,505 & 5,659 & .87 \\
\hline 2001 & 18.53 & 16.02 & 44,290 & 47,586 & 5,580 & 3,683 & .66 & 11,320 & 12,905 & 1.14 & 22,464 & 21,116 & .94 & 8,222 & 6,586 & .80 \\
\hline 2002 & 13.09 & 11.43 & 49,960 & 42,406 & 3,146 & 3,870 & 1.23 & 7,323 & 9,666 & 1.32 & 24,593 & 28,282 & 1.15 & 7,344 & 8,143 & 1.11 \\
\hline
\end{tabular}


Table 1. Precipitation, irrigation water use, acres irrigated, irrigation application rate by system type, and soil permeability for counties in the Groundwater Management Districts within the Kansas High Plains, 1991-2003.-Continued

[Precipitation data from Kansas State Climatologist (2005). Irrigation data from Kansas Water Office and Kansas Department of Agriculture (1993-2005). Soil permeability from U.S. Department of Agriculture (1994). Blue shading indicates trend is significant at 95 -percent confidence level ( $\mathrm{p} \leq 0.05$ ). Green shading indicates trend is significant at 90 -percent confidence level ( $\mathrm{p} \leq 0.10)$. acre-ft, acre-feet; acreft/acre, acre-feet per acre; NA, data not available; --, not computed]

\begin{tabular}{|c|c|c|c|c|c|c|c|c|c|c|c|c|c|c|c|c|}
\hline \multirow[b]{2}{*}{ Year } & \multirow[b]{2}{*}{$\begin{array}{l}\text { Annual } \\
\text { precip- } \\
\text { itation } \\
\text { (inches) }\end{array}$} & \multirow{2}{*}{$\begin{array}{l}\text { March- } \\
\text { October } \\
\text { precipi- } \\
\text { tation } \\
\text { (inches) }\end{array}$} & \multirow[b]{2}{*}{$\begin{array}{c}\text { Total } \\
\text { irrigation } \\
\text { water use } \\
\text { (acre-ft) }\end{array}$} & \multirow[b]{2}{*}{$\begin{array}{c}\text { Total acres } \\
\text { irrigated }\end{array}$} & \multicolumn{3}{|c|}{ Flood irrigation } & \multicolumn{3}{|c|}{ Center pivot irrigation } & \multicolumn{3}{|c|}{$\begin{array}{l}\text { Center pivot irrigation with } \\
\text { drop nozzle }\end{array}$} & \multicolumn{3}{|c|}{ Other irrigation systems } \\
\hline & & & & & $\begin{array}{c}\text { Total } \\
\text { acres } \\
\text { irrigated }\end{array}$ & $\begin{array}{c}\text { Water } \\
\text { used } \\
\text { (acre-ft) }\end{array}$ & $\begin{array}{l}\text { Rate of } \\
\text { irri- } \\
\text { gation } \\
\text { (acre- } \\
\text { ft/acre) }\end{array}$ & $\begin{array}{c}\text { Total } \\
\text { acres } \\
\text { irrigated }\end{array}$ & $\begin{array}{c}\text { Water } \\
\text { used } \\
\text { (acre-ft) }\end{array}$ & $\begin{array}{l}\text { Rate of } \\
\text { irri- } \\
\text { gation } \\
\text { (acre- } \\
\text { ft/acre) }\end{array}$ & $\begin{array}{c}\text { Total } \\
\text { acres } \\
\text { irrigated }\end{array}$ & $\begin{array}{c}\text { Water } \\
\text { used } \\
\text { (acre-ft) }\end{array}$ & $\begin{array}{l}\text { Rate of } \\
\text { irri- } \\
\text { gation } \\
\text { (acre- } \\
\text { ft/acre) }\end{array}$ & $\begin{array}{c}\text { Total } \\
\text { acres } \\
\text { irrigated }\end{array}$ & $\begin{array}{c}\text { Water } \\
\text { used } \\
\text { (acre-ft) }\end{array}$ & $\begin{array}{c}\text { Rate of } \\
\text { irri- } \\
\text { gation } \\
\text { (acre- } \\
\text { ft/acre) }\end{array}$ \\
\hline \multicolumn{17}{|c|}{ Morton County (estimated mean soil permeability $=2.5$ inches per hour) -Continued } \\
\hline 2003 & 19.22 & 17.38 & 39,507 & 42,786 & 1,954 & 1,583 & 0.81 & 11,127 & 10,682 & 0.96 & 24,391 & 21,464 & 0.88 & 5,314 & 5,779 & 1.09 \\
\hline Average & 18.06 & 16.16 & 57,928 & 49,545 & 15,763 & 19,860 & 1.10 & 11,257 & 13,619 & 1.22 & 14,301 & 14,795 & 1.09 & 9,325 & 10,792 & 1.16 \\
\hline p-value & .67 & -- & 0 & .08 & -- & -- & -- & -- & -- & -- & -- & -- & -- & -- & -- & -- \\
\hline slope & -.09 & -- & $-2,968$ & -532 & -- & -- & -- & -- & -- & -- & -- & -- & -- & -- & -- & -- \\
\hline \multicolumn{17}{|c|}{ Pawnee County (estimated mean soil permeability $=1.2$ inches per hour) } \\
\hline 1991 & 14.08 & 10.72 & 106,932 & 77,085 & 35,727 & 54,662 & 1.53 & 40,418 & 50,523 & 1.25 & NA & NA & NA & 940 & 1,747 & 1.86 \\
\hline 1992 & 32.14 & 26.55 & 45,543 & 70,536 & 29,419 & 18,240 & .62 & 38,004 & 25,463 & .67 & 1,734 & 1,231 & .71 & 1,379 & 609 & .44 \\
\hline 1993 & 38.05 & 30.99 & 31,209 & 64,354 & 23,952 & 10,778 & .45 & 38,512 & 19,256 & .50 & 723 & 289 & .40 & 1,167 & 885 & .76 \\
\hline 1994 & 14.83 & 11.72 & 90,564 & 74,522 & 31,653 & 39,250 & 1.24 & 40,513 & 49,021 & 1.21 & 423 & 326 & .77 & 1,933 & 1,968 & 1.02 \\
\hline 1995 & 24.52 & 22.38 & 75,112 & 77,576 & 33,064 & 31,411 & .95 & 39,920 & 39,122 & .98 & 3,124 & 3,186 & 1.02 & 1,468 & 1,393 & .95 \\
\hline 1996 & 32.01 & 28.58 & 53,184 & 74,372 & 27,290 & 20,468 & .75 & 40,253 & 28,177 & .70 & 4,932 & 3,600 & .73 & 1,897 & 939 & .49 \\
\hline 1997 & 27.34 & 23.64 & 53,515 & 75,503 & 24,555 & 18,171 & .74 & 28,019 & 19,333 & .69 & 19,278 & 13,880 & .72 & 3,651 & 2,131 & .58 \\
\hline 1998 & 25.25 & 20.51 & 77,546 & 80,600 & 24,263 & 22,807 & .94 & 22,154 & 21,711 & .98 & 29,720 & 28,531 & .96 & 4,463 & 4,497 & 1.01 \\
\hline 1999 & 24.92 & 22.32 & 67,624 & 80,135 & 22,619 & 18,774 & .83 & 21,726 & 18,684 & .86 & 31,173 & 27,121 & .87 & 4,617 & 3,045 & .66 \\
\hline 2000 & 26.74 & 23.08 & 84,802 & 79,215 & 19,061 & 21,348 & 1.12 & 16,664 & 18,830 & 1.13 & 37,624 & 38,753 & 1.03 & 5,866 & 5,870 & 1.00 \\
\hline 2001 & 24.35 & 20.94 & 82,947 & 79,534 & 18,317 & 19,233 & 1.05 & 11,907 & 11,907 & 1.00 & 43,371 & 45,973 & 1.06 & 5,939 & 5,834 & .98 \\
\hline 2002 & 17.37 & 15.98 & 94,792 & 79,512 & 16,944 & 19,655 & 1.16 & 11,734 & 14,198 & 1.21 & 43,154 & 51,353 & 1.19 & 7,680 & 9,586 & 1.25 \\
\hline 2003 & 19.06 & 17.12 & 83,221 & 74,912 & 12,124 & 15,276 & 1.26 & 9,905 & 10,995 & 1.11 & 45,815 & 49,938 & 1.09 & 7,068 & 7,012 & .99 \\
\hline Average & 24.66 & 21.12 & 72,846 & 75,989 & 24,538 & 23,852 & .97 & 27,671 & 25,171 & .95 & 21,756 & 22,015 & .88 & 3,698 & 3,501 & .95 \\
\hline p-value & .30 & -- & .20 & .10 & -- & -- & -- & -- & -- & -- & -- & -- & -- & -- & -- & -- \\
\hline slope & -.81 & -- & 2,374 & 450 & -- & -- & -- & -- & -- & -- & -- & -- & -- & -- & -- & -- \\
\hline \multicolumn{17}{|c|}{ Pratt County (estimated mean soil permeability $=4.1$ inches per hour) } \\
\hline 1991 & 28.72 & 24.61 & 97,761 & 71,656 & 1,838 & 2,831 & 1.54 & 67,299 & 91,527 & 1.36 & NA & NA & NA & 2,519 & 3,404 & 1.35 \\
\hline 1992 & 27.42 & 22.73 & 62,151 & 70,424 & 1,687 & 1,805 & 1.07 & 64,142 & 57,086 & .89 & 2,882 & 2,306 & .80 & 1,713 & 954 & .56 \\
\hline 1993 & 33.23 & 27.98 & 68,322 & 70,944 & 1,759 & 1,812 & 1.03 & 66,325 & 63,672 & .96 & 1,328 & 1,687 & 1.27 & 1,532 & 1,151 & .75 \\
\hline 1994 & 19.40 & 16.31 & 102,272 & 71,198 & 1,470 & 2,984 & 2.03 & 65,759 & 93,378 & 1.42 & 1,866 & 2,762 & 1.48 & 2,103 & 3,149 & 1.50 \\
\hline 1995 & 26.11 & 23.76 & 82,056 & 70,901 & 1,269 & 2,119 & 1.67 & 65,473 & 75,949 & 1.16 & 2,457 & 2,138 & .87 & 1,702 & 1,850 & 1.09 \\
\hline 1996 & 33.15 & 29.09 & 68,821 & 71,351 & 1,546 & 1,701 & 1.10 & 65,598 & 62,974 & .96 & 2,925 & 2,984 & 1.02 & 1,282 & 1,163 & .91 \\
\hline
\end{tabular}


Table 1. Precipitation, irrigation water use, acres irrigated, irrigation application rate by system type, and soil permeability for counties in the Groundwater Management Districts within the Kansas High Plains, 1991-2003.-Continued

[Precipitation data from Kansas State Climatologist (2005). Irrigation data from Kansas Water Office and Kansas Department of Agriculture (1993-2005). Soil permeability from U.S. Department of Agriculture (1994). Blue shading indicates trend is significant at 95-percent confidence level ( $\mathrm{p} \leq 0.05)$. Green shading indicates trend is significant at 90-percent confidence level ( $\mathrm{p} \leq 0.10)$. acre-ft, acre-feet; acreft/acre, acre-feet per acre; NA, data not available; --, not computed]

\begin{tabular}{|c|c|c|c|c|c|c|c|c|c|c|c|c|c|c|c|c|}
\hline \multirow[b]{2}{*}{ Year } & \multirow[b]{2}{*}{$\begin{array}{l}\text { Annual } \\
\text { precip- } \\
\text { itation } \\
\text { (inches) }\end{array}$} & \multirow{2}{*}{$\begin{array}{l}\text { March- } \\
\text { October } \\
\text { precipi- } \\
\text { tation } \\
\text { (inches) }\end{array}$} & \multirow[b]{2}{*}{$\begin{array}{c}\text { Total } \\
\text { irrigation } \\
\text { water use } \\
\text { (acre-ft) }\end{array}$} & \multirow[b]{2}{*}{$\begin{array}{c}\text { Total acres } \\
\text { irrigated }\end{array}$} & \multicolumn{3}{|c|}{ Flood irrigation } & \multicolumn{3}{|c|}{ Center pivot irrigation } & \multicolumn{3}{|c|}{$\begin{array}{l}\text { Center pivot irrigation with } \\
\text { drop nozzle }\end{array}$} & \multicolumn{3}{|c|}{ Other irrigation systems } \\
\hline & & & & & $\begin{array}{c}\text { Total } \\
\text { acres } \\
\text { irrigated }\end{array}$ & $\begin{array}{c}\text { Water } \\
\text { used } \\
\text { (acre-ft) }\end{array}$ & $\begin{array}{l}\text { Rate of } \\
\text { irri- } \\
\text { gation } \\
\text { (acre- } \\
\text { ft/acre) }\end{array}$ & $\begin{array}{c}\text { Total } \\
\text { acres } \\
\text { irrigated }\end{array}$ & $\begin{array}{c}\text { Water } \\
\text { used } \\
\text { (acre-ft) }\end{array}$ & $\begin{array}{l}\text { Rate of } \\
\text { irri- } \\
\text { gation } \\
\text { (acre- } \\
\text { ft/acre) }\end{array}$ & $\begin{array}{c}\text { Total } \\
\text { acres } \\
\text { irrigated }\end{array}$ & $\begin{array}{c}\text { Water } \\
\text { used } \\
\text { (acre-ft) }\end{array}$ & $\begin{array}{l}\text { Rate of } \\
\text { irri- } \\
\text { gation } \\
\text { (acre- } \\
\text { ft/acre) }\end{array}$ & $\begin{array}{c}\text { Total } \\
\text { acres } \\
\text { irrigated }\end{array}$ & $\begin{array}{c}\text { Water } \\
\text { used } \\
\text { (acre-ft) }\end{array}$ & $\begin{array}{l}\text { Rate of } \\
\text { irri- } \\
\text { gation } \\
\text { (acre- } \\
\text { ft/acre) }\end{array}$ \\
\hline \multicolumn{17}{|c|}{ Pratt County (estimated mean soil permeability $=4.1$ inches per hour) - Continued } \\
\hline 1997 & 34.67 & 28.46 & 57,970 & 73,131 & 1,349 & 1,187 & 0.88 & 46,462 & 35,311 & 0.76 & 24,132 & 20,754 & 0.86 & 1,188 & 718 & 0.60 \\
\hline 1998 & 24.23 & 19.06 & 92,301 & 75,305 & 1,183 & 1,644 & 1.39 & 37,791 & 45,727 & 1.21 & 34,652 & 42,968 & 1.24 & 1,679 & 1,962 & 1.17 \\
\hline 1999 & 28.26 & 25.52 & 92,628 & 80,233 & 1,165 & 1,445 & 1.24 & 39,427 & 44,553 & 1.13 & 37,541 & 44,674 & 1.19 & 2,100 & 1,958 & .93 \\
\hline 2000 & 34.92 & 29.51 & 91,301 & 81,714 & 920 & 1,040 & 1.13 & 28,988 & 32,177 & 1.11 & 49,397 & 55,325 & 1.12 & 2,409 & 2,760 & 1.15 \\
\hline 2001 & 15.01 & 12.75 & 109,599 & 82,459 & 957 & 1,522 & 1.59 & 22,725 & 29,543 & 1.30 & 55,908 & 74,917 & 1.34 & 2,869 & 3,618 & 1.26 \\
\hline 2002 & 28.14 & 24.83 & 99,366 & 83,607 & 672 & 753 & 1.12 & 22,221 & 26,443 & 1.19 & 57,178 & 68,042 & 1.19 & 3,536 & 4,129 & 1.17 \\
\hline 2003 & 24.43 & 21.40 & 110,278 & 84,191 & 887 & 1,153 & 1.30 & 23,435 & 30,934 & 1.32 & 57,402 & 74,623 & 1.30 & 2,467 & 3,568 & 1.45 \\
\hline Average & 27.51 & 23.54 & 87,294 & 75,932 & 1,285 & 1,692 & 1.31 & 47,357 & 53,021 & 1.14 & 27,306 & 32,765 & 1.14 & 2,085 & 2,337 & 1.12 \\
\hline p-value & .67 & -- & .06 & 0 & -- & -- & -- & -- & -- & -- & -- & -- & -- & -- & -- & -- \\
\hline slope & -.13 & -- & 3,349 & 1,285 & -- & -- & -- & -- & -- & -- & -- & -- & -- & -- & -- & -- \\
\hline \multicolumn{17}{|c|}{ Rawlins County (estimated mean soil permeability = 1.3 inches per hour) } \\
\hline 1991 & 22.55 & 19.27 & 19,212 & 16,397 & 4,826 & 6,805 & 1.41 & 6,899 & 6,416 & .93 & NA & NA & NA & 4,672 & 5,991 & 1.28 \\
\hline 1992 & 25.11 & 21.63 & 9,316 & 14,753 & 4,225 & 3,549 & .84 & 5,686 & 2,786 & .49 & 1,580 & 980 & .62 & 3,262 & 2,001 & .61 \\
\hline 1993 & 26.89 & 23.19 & 10,943 & 15,300 & 3,305 & 3,636 & 1.10 & 6,642 & 3,919 & .59 & 2,711 & 1,762 & .65 & 2,642 & 1,627 & .62 \\
\hline 1994 & 23.14 & 18.97 & 15,870 & 17,909 & 3,377 & 3,884 & 1.15 & 6,974 & 5,091 & .73 & 2,364 & 1,962 & .83 & 5,194 & 4,933 & .95 \\
\hline 1995 & 22.49 & 21.33 & 16,629 & 19,293 & 2,779 & 3,363 & 1.21 & 8,747 & 6,298 & .72 & 3,414 & 2,902 & .85 & 4,353 & 4,066 & .93 \\
\hline 1996 & 25.16 & 23.97 & 13,625 & 17,245 & 2,685 & 2,631 & .98 & 5,862 & 4,455 & .76 & 4,770 & 3,053 & .64 & 3,928 & 3,486 & .89 \\
\hline 1997 & 18.36 & 16.25 & 19,379 & 17,932 & 2,508 & 3,536 & 1.41 & 4,463 & 4,954 & 1.11 & 7,329 & 7,695 & 1.05 & 3,632 & 3,193 & .88 \\
\hline 1998 & 21.08 & 18.48 & 16,211 & 17,904 & 1,864 & 2,125 & 1.14 & 4,316 & 4,273 & .99 & 7,509 & 6,308 & .84 & 4,215 & 3,506 & .83 \\
\hline 1999 & 21.43 & 20.77 & 12,993 & 17,288 & 1,522 & 1,233 & .81 & 3,195 & 2,876 & .90 & 8,840 & 6,276 & .71 & 3,731 & 2,608 & .70 \\
\hline 2000 & 17.93 & 15.22 & 23,121 & 18,259 & 1,296 & 1,737 & 1.34 & 2,205 & 2,514 & 1.14 & 10,311 & 13,611 & 1.32 & 4,447 & 5,260 & 1.18 \\
\hline 2001 & 21.52 & 18.43 & 16,039 & 17,995 & 1,351 & 1,689 & 1.25 & 2,057 & 1,810 & .88 & 10,603 & 9,543 & .90 & 3,984 & 2,997 & .75 \\
\hline 2002 & 11.09 & 9.95 & 23,276 & 19,099 & 1,321 & 1,625 & 1.23 & 2,282 & 2,350 & 1.03 & 10,658 & 13,536 & 1.27 & 4,838 & 5,765 & 1.19 \\
\hline 2003 & 17.07 & 15.79 & 19,454 & 19,110 & 1,560 & 2,122 & 1.36 & 1,924 & 1,751 & .91 & 11,122 & 10,900 & .98 & 4,504 & 4,682 & 1.04 \\
\hline Average & 21.06 & 18.71 & 16,621 & 17,576 & 2,509 & 2,918 & 1.17 & 4,712 & 3,807 & .86 & 6,768 & 6,544 & .89 & 4,108 & 3,855 & .94 \\
\hline $\mathrm{p}$-value & .01 & -- & .06 & .01 & -- & -- & -- & -- & -- & -- & -- & -- & -- & -- & -- & -- \\
\hline slope & -.69 & -- & 752 & 249 & -- & -- & -- & -- & -- & -- & -- & -- & -- & -- & -- & -- \\
\hline
\end{tabular}


Table 1. Precipitation, irrigation water use, acres irrigated, irrigation application rate by system type, and soil permeability for counties in the Groundwater Management Districts within the Kansas High Plains, 1991-2003.-Continued

[Precipitation data from Kansas State Climatologist (2005). Irrigation data from Kansas Water Office and Kansas Department of Agriculture (1993-2005). Soil permeability from U.S. Department of Agriculture (1994). Blue shading indicates trend is significant at 95 -percent confidence level ( $\mathrm{p} \leq 0.05$ ). Green shading indicates trend is significant at 90 -percent confidence level ( $\mathrm{p} \leq 0.10)$. acre-ft, acre-feet; acre$\mathrm{ft} / \mathrm{acre}$, acre-feet per acre; NA, data not available; --, not computed]

\begin{tabular}{|c|c|c|c|c|c|c|c|c|c|c|c|c|c|c|c|c|}
\hline \multirow[b]{2}{*}{ Year } & \multirow[b]{2}{*}{$\begin{array}{l}\text { Annual } \\
\text { precip- } \\
\text { itation } \\
\text { (inches) }\end{array}$} & \multirow{2}{*}{$\begin{array}{l}\text { March- } \\
\text { October } \\
\text { precipi- } \\
\text { tation } \\
\text { (inches) }\end{array}$} & \multirow[b]{2}{*}{$\begin{array}{c}\text { Total } \\
\text { irrigation } \\
\text { water use } \\
\text { (acre-ft) }\end{array}$} & \multirow[b]{2}{*}{$\begin{array}{c}\text { Total acres } \\
\text { irrigated }\end{array}$} & \multicolumn{3}{|c|}{ Flood irrigation } & \multicolumn{3}{|c|}{ Center pivot irrigation } & \multicolumn{3}{|c|}{$\begin{array}{l}\text { Center pivot irrigation with } \\
\text { drop nozzle }\end{array}$} & \multicolumn{3}{|c|}{ Other irrigation systems } \\
\hline & & & & & $\begin{array}{c}\text { Total } \\
\text { acres } \\
\text { irrigated }\end{array}$ & $\begin{array}{c}\text { Water } \\
\text { used } \\
\text { (acre-ft) }\end{array}$ & $\begin{array}{l}\text { Rate of } \\
\text { irri- } \\
\text { gation } \\
\text { (acre- } \\
\text { ft/acre) }\end{array}$ & $\begin{array}{c}\text { Total } \\
\text { acres } \\
\text { irrigated }\end{array}$ & $\begin{array}{c}\text { Water } \\
\text { used } \\
\text { (acre-ft) }\end{array}$ & $\begin{array}{l}\text { Rate of } \\
\text { irri- } \\
\text { gation } \\
\text { (acre- } \\
\text { ft/acre) }\end{array}$ & $\begin{array}{c}\text { Total } \\
\text { acres } \\
\text { irrigated }\end{array}$ & $\begin{array}{c}\text { Water } \\
\text { used } \\
\text { (acre-ft) }\end{array}$ & $\begin{array}{c}\text { Rate of } \\
\text { irri- } \\
\text { gation } \\
\text { (acre- } \\
\text { ft/acre) }\end{array}$ & $\begin{array}{c}\text { Total } \\
\text { acres } \\
\text { irrigated }\end{array}$ & $\begin{array}{c}\text { Water } \\
\text { used } \\
\text { (acre-ft) }\end{array}$ & $\begin{array}{l}\text { Rate of } \\
\text { irri- } \\
\text { gation } \\
\text { (acre- } \\
\text { ft/acre) }\end{array}$ \\
\hline \multicolumn{17}{|c|}{ Reno County (estimated mean soil permeability $=4.7$ inches per hour) } \\
\hline 1991 & 25.65 & 22.30 & 38,133 & 33,755 & 3,457 & 3,492 & 1.01 & 26,698 & 31,237 & 1.17 & NA & NA & NA & 3,600 & 3,405 & 0.95 \\
\hline 1992 & 31.52 & 25.07 & 20,433 & 31,999 & 3,084 & 1,881 & 61 & 25,281 & 16,180 & .64 & 1,123 & 752 & .67 & 2,511 & 1,619 & .64 \\
\hline 1993 & 33.54 & 28.38 & 21,051 & 31,549 & 2,393 & 1,340 & .56 & 25,571 & 17,388 & .68 & 1,071 & 793 & .74 & 2,514 & 1,530 & .61 \\
\hline 1994 & 24.01 & 20.01 & 34,772 & 33,232 & 2,371 & 2,086 & .88 & 26,874 & 28,486 & 1.06 & 323 & 210 & .65 & 3,664 & 3,989 & 1.09 \\
\hline 1995 & 33.31 & 31.60 & 29,421 & 33,164 & 2,139 & 1,604 & .75 & 27,867 & 25,080 & .90 & 623 & 617 & .99 & 2,535 & 2,120 & .84 \\
\hline 1996 & 26.01 & 22.69 & 31,536 & 36,923 & 1,869 & 1,589 & .85 & 30,830 & 26,514 & .86 & 1,520 & 1,170 & .77 & 2,704 & 2,263 & .84 \\
\hline 1997 & 32.22 & 27.16 & 27,613 & 38,161 & 1,859 & 1,153 & .62 & 20,885 & 15,455 & .74 & 13,694 & 9,586 & .70 & 1,723 & 1,420 & .82 \\
\hline 1998 & 32.04 & 24.70 & 41,331 & 40,263 & 1,533 & 1,134 & .74 & 17,345 & 17,865 & 1.03 & 17,232 & 18,094 & 1.05 & 4,153 & 4,238 & 1.02 \\
\hline 1999 & 30.67 & 26.86 & 38,276 & 44,301 & 1,826 & 1,059 & .58 & 16,148 & 15,179 & .94 & 23,077 & 19,385 & .84 & 3,250 & 2,653 & .82 \\
\hline 2000 & 33.67 & 28.20 & 48,685 & 44,632 & 1,842 & 1,750 & .95 & 11,720 & 12,423 & 1.06 & 29,050 & 32,827 & 1.13 & 2,020 & 1,686 & .83 \\
\hline 2001 & 26.59 & 21.68 & 53,643 & 46,125 & 1,755 & 1,878 & 1.07 & 11,853 & 12,564 & 1.06 & 29,418 & 35,890 & 1.22 & 3,099 & 3,311 & 1.07 \\
\hline 2002 & 32.16 & 28.67 & 49,356 & 47,673 & 1,787 & 1,716 & .96 & 12,105 & 13,073 & 1.08 & 29,200 & 30,368 & 1.04 & 4,581 & 4,199 & .92 \\
\hline 2003 & 32.18 & 29.13 & 55,372 & 47,766 & 1,520 & 1,854 & 1.22 & 10,340 & 11,271 & 1.09 & 31,749 & 38,099 & 1.20 & 4,157 & 4,149 & 1.00 \\
\hline Average & 30.27 & 25.88 & 37,663 & 39,196 & 2,110 & 1,734 & .83 & 20,271 & 18,670 & .95 & 14,840 & 15,649 & .92 & 3,116 & 2,814 & .90 \\
\hline p-value & .43 & -- & 0 & 0 & -- & -- & -- & -- & -- & -- & -- & -- & -- & -- & -- & -- \\
\hline slope & .07 & -- & 2,859 & 1,618 & -- & -- & -- & -- & -- & -- & -- & -- & -- & -- & -- & -- \\
\hline \multicolumn{17}{|c|}{ Rice County (estimated mean soil permeability $=1.8$ inches per hour) } \\
\hline 1991 & 18.89 & 15.39 & 27,412 & 21,182 & 2,087 & 2,880 & 1.38 & 18,402 & 23,555 & 1.28 & NA & NA & NA & 693 & 978 & 1.41 \\
\hline 1992 & 33.86 & 29.18 & 10,793 & 20,546 & 1,714 & 1,063 & .62 & 17,344 & 8,672 & .50 & 1,124 & 652 & .58 & 364 & 406 & 1.12 \\
\hline 1993 & 39.15 & 33.31 & 9,966 & 19,863 & 1,257 & 528 & .42 & 17,119 & 8,560 & .50 & 1,045 & 784 & .75 & 442 & 94 & .21 \\
\hline 1994 & 17.56 & 14.41 & 25,946 & 21,450 & 1,598 & 2,125 & 1.33 & 17,809 & 21,549 & 1.21 & 1,360 & 1,836 & 1.35 & 683 & 436 & .64 \\
\hline 1995 & 31.48 & 29.67 & 17,621 & 20,314 & 1,252 & 1,064 & .85 & 17,032 & 14,477 & .85 & 1,421 & 1,336 & .94 & 609 & 744 & 1.22 \\
\hline 1996 & 25.97 & 22.12 & 19,344 & 21,446 & 1,358 & 1,317 & .97 & 17,893 & 15,746 & .88 & 2,030 & 2,050 & 1.01 & 165 & 230 & 1.39 \\
\hline 1997 & 3.46 & 25.83 & 17,739 & 21,752 & 1,120 & 1,109 & .99 & 12,555 & 10,295 & .82 & 7,767 & 6,058 & .78 & 310 & 277 & .89 \\
\hline 1998 & 28.93 & 24.07 & 18,857 & 21,815 & 1,236 & 1,075 & .87 & 12,563 & 10,553 & .84 & 7,703 & 6,933 & .90 & 313 & 296 & .95 \\
\hline 1999 & 26.46 & 23.32 & 20,041 & 22,803 & 1,276 & 1,059 & .83 & 9,608 & 7,879 & .82 & 11,589 & 10,894 & .94 & 330 & 209 & .63 \\
\hline 2000 & 31.22 & 26.02 & 23,217 & 22,852 & 1,089 & 904 & .83 & 8,688 & 8,080 & .93 & 11,835 & 12,900 & 1.09 & 1,240 & 1,333 & 1.07 \\
\hline 2001 & 22.42 & 17.96 & 28,147 & 23,260 & 1,455 & 1,644 & 1.13 & 6,974 & 7,741 & 1.11 & 14,428 & 18,035 & 1.25 & 403 & 727 & 1.80 \\
\hline 2002 & 27.73 & 25.57 & 23,516 & 23,207 & 1,348 & 1,375 & 1.02 & 5,638 & 5,356 & .95 & 15,107 & 15,409 & 1.02 & 1,114 & 1,376 & 1.24 \\
\hline
\end{tabular}


Table 1. Precipitation, irrigation water use, acres irrigated, irrigation application rate by system type, and soil permeability for counties in the Groundwater Management Districts within the Kansas High Plains, 1991-2003.-Continued

[Precipitation data from Kansas State Climatologist (2005). Irrigation data from Kansas Water Office and Kansas Department of Agriculture (1993-2005). Soil permeability from U.S. Department of Agriculture (1994). Blue shading indicates trend is significant at 95-percent confidence level ( $\mathrm{p} \leq 0.05)$. Green shading indicates trend is significant at 90-percent confidence level ( $\mathrm{p} \leq 0.10)$. acre-ft, acre-feet; acreft/acre, acre-feet per acre; NA, data not available; --, not computed]

\begin{tabular}{|c|c|c|c|c|c|c|c|c|c|c|c|c|c|c|c|c|}
\hline \multirow[b]{2}{*}{ Year } & \multirow[b]{2}{*}{$\begin{array}{l}\text { Annual } \\
\text { precip- } \\
\text { itation } \\
\text { (inches) }\end{array}$} & \multirow{2}{*}{$\begin{array}{l}\text { March- } \\
\text { October } \\
\text { precipi- } \\
\text { tation } \\
\text { (inches) }\end{array}$} & \multirow[b]{2}{*}{$\begin{array}{c}\text { Total } \\
\text { irrigation } \\
\text { water use } \\
\text { (acre-ft) }\end{array}$} & \multirow[b]{2}{*}{$\begin{array}{c}\text { Total acres } \\
\text { irrigated }\end{array}$} & \multicolumn{3}{|c|}{ Flood irrigation } & \multicolumn{3}{|c|}{ Center pivot irrigation } & \multicolumn{3}{|c|}{$\begin{array}{l}\text { Center pivot irrigation with } \\
\text { drop nozzle }\end{array}$} & \multicolumn{3}{|c|}{ Other irrigation systems } \\
\hline & & & & & $\begin{array}{c}\text { Total } \\
\text { acres } \\
\text { irrigated }\end{array}$ & $\begin{array}{c}\text { Water } \\
\text { used } \\
\text { (acre-ft) }\end{array}$ & $\begin{array}{l}\text { Rate of } \\
\text { irri- } \\
\text { gation } \\
\text { (acre- } \\
\text { ft/acre) }\end{array}$ & $\begin{array}{c}\text { Total } \\
\text { acres } \\
\text { irrigated }\end{array}$ & $\begin{array}{c}\text { Water } \\
\text { used } \\
\text { (acre-ft) }\end{array}$ & $\begin{array}{l}\text { Rate of } \\
\text { irri- } \\
\text { gation } \\
\text { (acre- } \\
\text { ft/acre) }\end{array}$ & $\begin{array}{c}\text { Total } \\
\text { acres } \\
\text { irrigated }\end{array}$ & $\begin{array}{c}\text { Water } \\
\text { used } \\
\text { (acre-ft) }\end{array}$ & $\begin{array}{l}\text { Rate of } \\
\text { irri- } \\
\text { gation } \\
\text { (acre- } \\
\text { ft/acre) }\end{array}$ & $\begin{array}{c}\text { Total } \\
\text { acres } \\
\text { irrigated }\end{array}$ & $\begin{array}{c}\text { Water } \\
\text { used } \\
\text { (acre-ft) }\end{array}$ & $\begin{array}{l}\text { Rate of } \\
\text { irri- } \\
\text { gation } \\
\text { (acre- } \\
\mathrm{ft} / \text { acre) }\end{array}$ \\
\hline \multicolumn{17}{|c|}{ Rice County (estimated mean soil permeability $=1.8$ inches per hour) -Continued } \\
\hline 2003 & 24.01 & 21.96 & 28,993 & 22,801 & 1,054 & 1,423 & 1.35 & 3,085 & 3,579 & 1.16 & 17,581 & 22,504 & 1.28 & 1,081 & 1,487 & 1.38 \\
\hline Average & 27.55 & 23.75 & 20,892 & 21,792 & 1,373 & 1,351 & .97 & 12,670 & 11,234 & .91 & 7,749 & 8,283 & .99 & 596 & 661 & 1.11 \\
\hline p-value & .43 & -- & .03 & 0 & -- & -- & -- & -- & -- & -- & -- & -- & -- & -- & -- & -- \\
\hline slope & -.54 & -- & 1,161 & 237 & -- & -- & -- & -- & -- & -- & -- & -- & -- & -- & -- & -- \\
\hline \multicolumn{17}{|c|}{ Scott County (estimated mean soil permeability $=1.2$ inches per hour) } \\
\hline 1991 & 21.53 & 18.07 & 89,217 & 66,737 & 62,014 & 82,479 & 1.33 & 1,575 & 2,000 & 1.27 & NA & NA & NA & 3,148 & 4,738 & 1.51 \\
\hline 1992 & 22.29 & 17.62 & 68,154 & 60,801 & 57,975 & 65,512 & 1.13 & 1,614 & 1,614 & 1.00 & 352 & 197 & .56 & 860 & 831 & .97 \\
\hline 1993 & 28.23 & 23.61 & 45,229 & 52,291 & 45,434 & 39,982 & .88 & 3,020 & 2,325 & .77 & 1,210 & 750 & .62 & 2,627 & 2,172 & .83 \\
\hline 1994 & 18.59 & 15.55 & 68,869 & 58,816 & 48,409 & 56,639 & 1.17 & 2,701 & 3,052 & 1.13 & 2,354 & 2,260 & .96 & 5,352 & 6,919 & 1.29 \\
\hline 1995 & 17.69 & 17.03 & 66,414 & 61,306 & 44,264 & 50,018 & 1.13 & 5,973 & 6,451 & 1.08 & 3,371 & 2,865 & .85 & 7,698 & 7,079 & .92 \\
\hline 1996 & 25.13 & 23.09 & 53,285 & 59,206 & 40,126 & 35,311 & .88 & 7,685 & 6,763 & .88 & 3,716 & 3,307 & .89 & 7,679 & 7,905 & 1.03 \\
\hline 1997 & 21.02 & 19.02 & 52,927 & 59,997 & 35,963 & 32,007 & .89 & 7,441 & 6,027 & .81 & 6,919 & 6,504 & .94 & 9,674 & 8,389 & .87 \\
\hline 1998 & 18.41 & 15.13 & 52,090 & 60,720 & 29,596 & 26,932 & .91 & 12,280 & 10,070 & .82 & 6,413 & 5,323 & .83 & 12,431 & 9,765 & .79 \\
\hline 1999 & 18.66 & 17.70 & 53,342 & 57,992 & 25,324 & 26,084 & 1.03 & 12,588 & 10,448 & .83 & 9,724 & 7,390 & .76 & 10,356 & 9,420 & .91 \\
\hline 2000 & 18.26 & 15.85 & 65,186 & 61,174 & 23,908 & 26,299 & 1.10 & 6,199 & 7,129 & 1.15 & 20,765 & 21,180 & 1.02 & 10,302 & 10,578 & 1.03 \\
\hline 2001 & 17.80 & 16.04 & 51,564 & 58,450 & 19,041 & 18,279 & .96 & 6,868 & 6,525 & .95 & 21,034 & 19,562 & .93 & 11,507 & 7,198 & .63 \\
\hline 2002 & 13.18 & 12.60 & 70,469 & 61,338 & 18,278 & 21,568 & 1.18 & 5,739 & 7,059 & 1.23 & 24,054 & 28,384 & 1.18 & 13,267 & 13,458 & 1.01 \\
\hline 2003 & 16.95 & 16.09 & 61,369 & 59,597 & 17,237 & 17,926 & 1.04 & 5,158 & 5,622 & 1.09 & 25,985 & 25,205 & .97 & 11,217 & 12,615 & 1.12 \\
\hline Average & 19.83 & 17.49 & 61,393 & 59,879 & 35,967 & 38,387 & 1.05 & 6,065 & 5,776 & 1.00 & 10,491 & 10,244 & .88 & 8,163 & 7,774 & .95 \\
\hline p-value & .01 & -- & .43 & .95 & -- & -- & -- & -- & -- & -- & -- & -- & -- & -- & -- & -- \\
\hline slope & -.50 & -- & -589 & -20 & -- & -- & -- & -- & -- & -- & -- & -- & -- & -- & -- & -- \\
\hline \multicolumn{17}{|c|}{ Sedgwick County (estimated mean soil permeability = 2.9 inches per hour) } \\
\hline 1991 & 25.40 & 20.54 & 42,425 & 35,420 & 13,912 & 17,807 & 1.28 & 18,450 & 21,771 & 1.18 & NA & NA & NA & 3,058 & 2,847 & .93 \\
\hline 1992 & 35.51 & 27.81 & 25,462 & 33,004 & 11,301 & 9,380 & .83 & 18,529 & 12,970 & .70 & 713 & 435 & .61 & 2,461 & 2,677 & 1.09 \\
\hline 1993 & 37.44 & 32.07 & 28,513 & 34,379 & 11,942 & 10,867 & .91 & 19,256 & 15,020 & .78 & 295 & 224 & .76 & 2,886 & 2,402 & .83 \\
\hline 1994 & 25.82 & 21.34 & 37,948 & 35,423 & 12,498 & 14,498 & 1.16 & 18,823 & 19,199 & 1.02 & 585 & 509 & .87 & 3,517 & 3,742 & 1.06 \\
\hline 1995 & 36.38 & 34.55 & 30,594 & 36,317 & 12,471 & 10,974 & .88 & 19,793 & 16,428 & .83 & 1,117 & 525 & .47 & 2,936 & 2,666 & .91 \\
\hline 1996 & 30.57 & 26.18 & 32,879 & 36,763 & 11,359 & 11,132 & .98 & 22,039 & 18,733 & .85 & 495 & 515 & 1.04 & 2,870 & 2,500 & .87 \\
\hline
\end{tabular}


Table 1. Precipitation, irrigation water use, acres irrigated, irrigation application rate by system type, and soil permeability for counties in the Groundwater Management Districts within the Kansas High Plains, 1991-2003.-Continued

[Precipitation data from Kansas State Climatologist (2005). Irrigation data from Kansas Water Office and Kansas Department of Agriculture (1993-2005). Soil permeability from U.S. Department of Agriculture (1994). Blue shading indicates trend is significant at 95 -percent confidence level ( $\mathrm{p} \leq 0.05$ ). Green shading indicates trend is significant at 90 -percent confidence level ( $\mathrm{p} \leq 0.10)$. acre-ft, acre-feet; acreft/acre, acre-feet per acre; NA, data not available; --, not computed]

\begin{tabular}{|c|c|c|c|c|c|c|c|c|c|c|c|c|c|c|c|c|}
\hline \multirow[b]{2}{*}{ Year } & \multirow[b]{2}{*}{$\begin{array}{l}\text { Annual } \\
\text { precip- } \\
\text { itation } \\
\text { (inches) }\end{array}$} & \multirow{2}{*}{$\begin{array}{l}\text { March- } \\
\text { October } \\
\text { precipi- } \\
\text { tation } \\
\text { (inches) }\end{array}$} & \multirow[b]{2}{*}{$\begin{array}{c}\text { Total } \\
\text { irrigation } \\
\text { water use } \\
\text { (acre-ft) }\end{array}$} & \multirow[b]{2}{*}{$\begin{array}{c}\text { Total acres } \\
\text { irrigated }\end{array}$} & \multicolumn{3}{|c|}{ Flood irrigation } & \multicolumn{3}{|c|}{ Center pivot irrigation } & \multicolumn{3}{|c|}{$\begin{array}{l}\text { Center pivot irrigation with } \\
\text { drop nozzle }\end{array}$} & \multicolumn{3}{|c|}{ Other irrigation systems } \\
\hline & & & & & $\begin{array}{c}\text { Total } \\
\text { acres } \\
\text { irrigated }\end{array}$ & $\begin{array}{c}\text { Water } \\
\text { used } \\
\text { (acre-ft) }\end{array}$ & $\begin{array}{l}\text { Rate of } \\
\text { irri- } \\
\text { gation } \\
\text { (acre- } \\
\text { ft/acre) }\end{array}$ & $\begin{array}{c}\text { Total } \\
\text { acres } \\
\text { irrigated }\end{array}$ & $\begin{array}{c}\text { Water } \\
\text { used } \\
\text { (acre-ft) }\end{array}$ & $\begin{array}{l}\text { Rate of } \\
\text { irri- } \\
\text { gation } \\
\text { (acre- } \\
\text { ft/acre) }\end{array}$ & $\begin{array}{c}\text { Total } \\
\text { acres } \\
\text { irrigated }\end{array}$ & $\begin{array}{c}\text { Water } \\
\text { used } \\
\text { (acre-ft) }\end{array}$ & $\begin{array}{l}\text { Rate of } \\
\text { irri- } \\
\text { gation } \\
\text { (acre- } \\
\text { ft/acre) }\end{array}$ & $\begin{array}{c}\text { Total } \\
\text { acres } \\
\text { irrigated }\end{array}$ & $\begin{array}{c}\text { Water } \\
\text { used } \\
\text { (acre-ft) }\end{array}$ & $\begin{array}{c}\text { Rate of } \\
\text { irri- } \\
\text { gation } \\
\text { (acre- } \\
\text { ft/acre) }\end{array}$ \\
\hline \multicolumn{17}{|c|}{ Sedgwick County (estimated mean soil permeability $=2.9$ inches per hour) - Continued } \\
\hline 1997 & 34.70 & 28.14 & 27,267 & 37,063 & 10,802 & 9,614 & 0.89 & 16,203 & 10,694 & 0.66 & 6,816 & 4,499 & 0.66 & 3,242 & 2,460 & 0.76 \\
\hline 1998 & 34.91 & 27.13 & 37,601 & 38,113 & 10,210 & 11,435 & 1.12 & 17,622 & 16,036 & .91 & 6,428 & 6,557 & 1.02 & 3,853 & 3,574 & .93 \\
\hline 1999 & 41.10 & 34.84 & 31,010 & 38,730 & 10,565 & 9,931 & .94 & 16,075 & 11,735 & .73 & 8,329 & 6,663 & .80 & 3,761 & 2,681 & .71 \\
\hline 2000 & 30.64 & 25.63 & 37,079 & 38,084 & 10,180 & 11,809 & 1.16 & 10,326 & 8,984 & .87 & 13,882 & 12,494 & .90 & 3,696 & 3,793 & 1.03 \\
\hline 2001 & 28.64 & 22.43 & 42,954 & 38,554 & 9,840 & 12,398 & 1.26 & 10,369 & 10,887 & 1.05 & 13,749 & 14,436 & 1.05 & 4,596 & 5,232 & 1.14 \\
\hline 2002 & 34.80 & 31.38 & 42,381 & 41,243 & 9,944 & 12,231 & 1.23 & 7,188 & 6,757 & .94 & 18,532 & 17,605 & .95 & 5,579 & 5,788 & 1.04 \\
\hline 2003 & 31.08 & 28.13 & 42,752 & 39,432 & 9,115 & 11,211 & 1.23 & 6,283 & 6,409 & 1.02 & 17,668 & 18,728 & 1.06 & 6,366 & 6,404 & 1.01 \\
\hline Average & 32.84 & 27.70 & 35,297 & 37,117 & 11,088 & 11,791 & 1.07 & 15,458 & 13,509 & .89 & 7,384 & 6,932 & .85 & 3,755 & 3,597 & .96 \\
\hline p-value & .95 & -- & .08 & 0 & -- & -- & -- & -- & -- & -- & -- & -- & -- & -- & -- & -- \\
\hline slope & .02 & -- & 1,260 & 529 & -- & -- & -- & -- & -- & -- & -- & -- & -- & -- & -- & -- \\
\hline \multicolumn{17}{|c|}{ Seward County (estimated mean soil permeability $=3.1$ inches per hour) } \\
\hline 1991 & 21.77 & 17.98 & 199,783 & 109,896 & 57,574 & 112,845 & 1.96 & 41,146 & 69,948 & 1.70 & NA & NA & NA & 11,176 & 16,989 & 1.52 \\
\hline 1992 & 19.40 & 14.39 & 174,163 & 109,293 & 56,447 & 94,831 & 1.68 & 40,783 & 63,621 & 1.56 & 4,221 & 5,150 & 1.22 & 7,842 & 10,561 & 1.35 \\
\hline 1993 & 22.94 & 18.56 & 150,954 & 107,865 & 53,299 & 77,284 & 1.45 & 40,564 & 57,601 & 1.42 & 2,676 & 2,783 & 1.04 & 11,326 & 13,287 & 1.17 \\
\hline 1994 & 20.70 & 18.84 & 165,559 & 109,022 & 50,035 & 81,557 & 1.63 & 42,542 & 59,984 & 1.41 & 2,572 & 3,652 & 1.42 & 13,873 & 20,365 & 1.47 \\
\hline 1995 & 15.47 & 14.32 & 168,353 & 108,298 & 41,102 & 69,873 & 1.70 & 45,942 & 70,291 & 1.53 & 1,395 & 2,609 & 1.87 & 19,859 & 25,579 & 1.29 \\
\hline 1996 & 23.45 & 21.62 & 164,911 & 108,727 & 36,566 & 57,043 & 1.56 & 52,663 & 80,574 & 1.53 & 3,265 & 4,734 & 1.45 & 16,233 & 22,559 & 1.39 \\
\hline 1997 & 17.86 & 15.76 & 151,370 & 116,582 & 31,919 & 43,410 & 1.36 & 41,706 & 55,469 & 1.33 & 21,701 & 29,296 & 1.35 & 21,256 & 23,195 & 1.09 \\
\hline 1998 & 25.85 & 24.81 & 157,773 & 123,217 & 23,808 & 30,712 & 1.29 & 38,916 & 52,537 & 1.35 & 33,082 & 41,683 & 1.26 & 27,411 & 32,841 & 1.20 \\
\hline 1999 & 20.58 & 19.61 & 135,520 & 122,761 & 19,427 & 22,341 & 1.15 & 36,815 & 40,497 & 1.10 & 38,220 & 43,189 & 1.13 & 28,299 & 29,493 & 1.04 \\
\hline 2000 & 20.92 & 20.17 & 161,124 & 122,163 & 19,261 & 27,351 & 1.42 & 27,580 & 36,406 & 1.32 & 50,435 & 66,070 & 1.31 & 24,887 & 31,298 & 1.26 \\
\hline 2001 & 15.64 & 13.42 & 157,073 & 126,340 & 13,739 & 17,174 & 1.25 & 22,650 & 30,125 & 1.33 & 60,664 & 79,470 & 1.31 & 29,287 & 30,305 & 1.03 \\
\hline 2002 & 15.14 & 13.15 & 204,566 & 130,913 & 12,401 & 20,338 & 1.64 & 19,767 & 29,255 & 1.48 & 57,001 & 94,622 & 1.66 & 41,744 & 60,352 & 1.45 \\
\hline 2003 & 19.77 & 18.30 & 147,422 & 117,791 & 9,518 & 11,326 & 1.19 & 18,824 & 21,648 & 1.15 & 62,359 & 78,572 & 1.26 & 27,090 & 35,876 & 1.32 \\
\hline Average & 19.96 & 17.76 & 164,505 & 116,374 & 32,700 & 51,237 & 1.48 & 36,146 & 51,381 & 1.40 & 28,133 & 37,652 & 1.36 & 21,560 & 27,131 & 1.26 \\
\hline p-value & .30 & -- & .13 & .02 & -- & -- & -- & -- & -- & -- & -- & -- & -- & -- & -- & -- \\
\hline slope & -.31 & -- & $-1,941$ & 1,877 & -- & -- & -- & -- & -- & -- & -- & -- & -- & -- & -- & -- \\
\hline
\end{tabular}


Table 1. Precipitation, irrigation water use, acres irrigated, irrigation application rate by system type, and soil permeability for counties in the Groundwater Management Districts within the Kansas High Plains, 1991-2003.-Continued

[Precipitation data from Kansas State Climatologist (2005). Irrigation data from Kansas Water Office and Kansas Department of Agriculture (1993-2005). Soil permeability from U.S. Department of Agriculture (1994). Blue shading indicates trend is significant at 95-percent confidence level $(\mathrm{p}<0.05)$. Green shading indicates trend is significant at 90 -percent confidence level $(\mathrm{p}<0.10)$. acre-ft, acre-feet; acreft/acre, acre-feet per acre; NA, data not available; --, not computed]

\begin{tabular}{|c|c|c|c|c|c|c|c|c|c|c|c|c|c|c|c|c|}
\hline \multirow[b]{2}{*}{ Year } & \multirow[b]{2}{*}{$\begin{array}{l}\text { Annual } \\
\text { precip- } \\
\text { itation } \\
\text { (inches) }\end{array}$} & \multirow{2}{*}{$\begin{array}{l}\text { March- } \\
\text { October } \\
\text { precipi- } \\
\text { tation } \\
\text { (inches) }\end{array}$} & \multirow[b]{2}{*}{$\begin{array}{c}\text { Total } \\
\text { irrigation } \\
\text { water use } \\
\text { (acre-ft) }\end{array}$} & \multirow[b]{2}{*}{$\begin{array}{c}\text { Total acres } \\
\text { irrigated }\end{array}$} & \multicolumn{3}{|c|}{ Flood irrigation } & \multicolumn{3}{|c|}{ Center pivot irrigation } & \multicolumn{3}{|c|}{$\begin{array}{l}\text { Center pivot irrigation with } \\
\text { drop nozzle }\end{array}$} & \multicolumn{3}{|c|}{ Other irrigation systems } \\
\hline & & & & & $\begin{array}{c}\text { Total } \\
\text { acres } \\
\text { irrigated }\end{array}$ & $\begin{array}{c}\text { Water } \\
\text { used } \\
\text { (acre-ft) }\end{array}$ & $\begin{array}{l}\text { Rate of } \\
\text { irri- } \\
\text { gation } \\
\text { (acre- } \\
\text { ft/acre) }\end{array}$ & $\begin{array}{c}\text { Total } \\
\text { acres } \\
\text { irrigated }\end{array}$ & $\begin{array}{c}\text { Water } \\
\text { used } \\
\text { (acre-ft) }\end{array}$ & $\begin{array}{l}\text { Rate of } \\
\text { irri- } \\
\text { gation } \\
\text { (acre- } \\
\text { ft/acre) }\end{array}$ & $\begin{array}{c}\text { Total } \\
\text { acres } \\
\text { irrigated }\end{array}$ & $\begin{array}{c}\text { Water } \\
\text { used } \\
\text { (acre-ft) }\end{array}$ & $\begin{array}{c}\text { Rate of } \\
\text { irri- } \\
\text { gation } \\
\text { (acre- } \\
\text { ft/acre) }\end{array}$ & $\begin{array}{c}\text { Total } \\
\text { acres } \\
\text { irrigated }\end{array}$ & $\begin{array}{c}\text { Water } \\
\text { used } \\
\text { (acre-ft) }\end{array}$ & $\begin{array}{l}\text { Rate of } \\
\text { irri- } \\
\text { gation } \\
\text { (acre- } \\
\text { ft/acre) }\end{array}$ \\
\hline \multicolumn{17}{|c|}{ Sheridan County (estimated mean soil permeability $=1.3$ inches per hour) } \\
\hline 1991 & 20.18 & 17.11 & 106,056 & 72,711 & 25,236 & 41,892 & 1.66 & 37,491 & 49,113 & 1.31 & NA & NA & NA & 9,984 & 15,051 & 1.51 \\
\hline 1992 & 30.04 & 25.73 & 52,518 & 67,069 & 19,013 & 17,682 & .93 & 30,634 & 21,750 & .71 & 8,441 & 6,331 & .75 & 8,981 & 6,755 & .75 \\
\hline 1993 & 33.03 & 28.77 & 37,556 & 67,138 & 16,780 & 12,082 & .72 & 32,319 & 16,160 & .50 & 11,920 & 6,556 & .55 & 6,119 & 2,759 & .45 \\
\hline 1994 & 20.05 & 16.87 & 82,758 & 71,739 & 16,402 & 22,799 & 1.39 & 31,462 & 34,294 & 1.09 & 15,350 & 17,039 & 1.11 & 8,525 & 8,628 & 1.01 \\
\hline 1995 & 23.66 & 22.65 & 85,854 & 71,711 & 14,580 & 20,120 & 1.38 & 31,471 & 36,821 & 1.17 & 17,075 & 18,953 & 1.11 & 8,585 & 9,959 & 1.16 \\
\hline 1996 & 24.40 & 22.83 & 72,327 & 73,368 & 11,889 & 14,029 & 1.18 & 31,480 & 30,221 & .96 & 18,485 & 16,821 & .91 & 11,514 & 11,256 & .98 \\
\hline 1997 & 20.87 & 18.41 & 71,798 & 75,041 & 9,184 & 9,735 & 1.06 & 26,393 & 24,809 & .94 & 29,963 & 27,566 & .92 & 9,501 & 9,688 & 1.02 \\
\hline 1998 & 20.49 & 16.15 & 71,006 & 74,876 & 6,906 & 8,011 & 1.16 & 21,180 & 19,909 & .94 & 36,458 & 33,177 & .91 & 10,332 & 9,909 & .96 \\
\hline 1999 & 22.37 & 21.42 & 62,029 & 75,310 & 5,637 & 5,468 & .97 & 21,950 & 17,780 & .81 & 35,215 & 28,524 & .81 & 12,508 & 10,258 & .82 \\
\hline 2000 & 18.16 & 15.94 & 97,567 & 76,762 & 5,234 & 7,799 & 1.49 & 13,912 & 17,112 & 1.23 & 47,070 & 59,779 & 1.27 & 10,546 & 12,878 & 1.22 \\
\hline 2001 & 22.60 & 19.86 & 81,265 & 74,168 & 3,197 & 3,900 & 1.22 & 11,416 & 12,558 & 1.10 & 48,340 & 52,207 & 1.08 & 11,215 & 12,600 & 1.12 \\
\hline 2002 & 10.44 & 9.82 & 103,788 & 76,432 & 3,573 & 5,324 & 1.49 & 9,683 & 12,878 & 1.33 & 51,612 & 71,225 & 1.38 & 11,564 & 14,361 & 1.24 \\
\hline 2003 & 13.86 & 12.83 & 98,120 & 77,645 & 2,475 & 3,539 & 1.43 & 10,072 & 12,187 & 1.21 & 54,668 & 69,975 & 1.28 & 10,430 & 12,418 & 1.19 \\
\hline Average & 21.55 & 19.11 & 78,665 & 73,382 & 10,777 & 13,260 & 1.24 & 23,805 & 23,507 & 1.02 & 31,216 & 34,013 & 1.01 & 9,985 & 10,501 & 1.05 \\
\hline p-value & .06 & -- & .36 & 0 & -- & -- & -- & -- & -- & -- & -- & -- & -- & -- & -- & -- \\
\hline slope & -1.13 & -- & 2,515 & 694 & -- & -- & -- & -- & -- & -- & -- & -- & -- & -- & -- & -- \\
\hline \multicolumn{17}{|c|}{ Sherman County (estimated mean soil permeability $=1.3$ inches per hour) } \\
\hline 1991 & 23.24 & 20.39 & 134,181 & 107,024 & 40,720 & 63,608 & 1.56 & 59,639 & 62,064 & 1.04 & -- & -- & -- & 6,665 & 8,509 & 1.28 \\
\hline 1992 & 21.93 & 20.53 & 90,380 & 103,480 & 32,740 & 37,137 & 1.13 & 54,167 & 41,460 & .77 & 11,002 & 7,417 & .67 & 5,571 & 4,365 & .78 \\
\hline 1993 & 27.98 & 26.00 & 88,641 & 105,369 & 31,124 & 35,884 & 1.15 & 54,332 & 39,668 & .73 & 13,784 & 8,666 & .63 & 6,129 & 4,424 & .72 \\
\hline 1994 & 15.84 & 13.65 & 113,957 & 110,456 & 27,033 & 34,438 & 1.27 & 56,455 & 55,350 & .98 & 18,095 & 16,771 & .93 & 8,873 & 7,398 & .83 \\
\hline 1995 & 20.82 & 19.80 & 97,823 & 103,751 & 20,617 & 24,577 & 1.19 & 61,606 & 55,726 & .90 & 13,782 & 11,795 & .86 & 7,746 & 5,725 & .74 \\
\hline 1996 & 19.23 & 18.69 & 116,340 & 114,599 & 18,411 & 22,801 & 1.24 & 68,502 & 67,305 & .98 & 19,045 & 17,518 & .92 & 8,641 & 8,716 & 1.01 \\
\hline 1997 & 18.67 & 16.44 & 132,015 & 116,825 & 15,828 & 20,067 & 1.27 & 44,234 & 46,913 & 1.06 & 50,580 & 58,426 & 1.16 & 6,183 & 6,609 & 1.07 \\
\hline 1998 & 18.31 & 15.33 & 120,595 & 116,611 & 12,206 & 12,476 & 1.02 & 29,983 & 29,972 & 1.00 & 67,164 & 70,830 & 1.05 & 7,258 & 7,316 & 1.01 \\
\hline 1999 & 20.29 & 19.27 & 104,481 & 114,015 & 9,419 & 9,949 & 1.06 & 24,774 & 20,925 & .84 & 70,224 & 65,368 & .93 & 9,598 & 8,240 & .86 \\
\hline 2000 & 16.82 & 15.17 & 142,948 & 115,596 & 7,048 & 10,592 & 1.50 & 18,452 & 22,779 & 1.23 & 83,083 & 101,880 & 1.23 & 7,013 & 7,696 & 1.10 \\
\hline 2001 & 18.35 & 15.35 & 128,139 & 114,314 & 6,378 & 7,851 & 1.23 & 16,310 & 19,093 & 1.17 & 85,417 & 94,619 & 1.11 & 6,209 & 6,576 & 1.06 \\
\hline 2002 & 11.02 & 10.43 & 156,184 & 115,501 & 4,869 & 7,897 & 1.62 & 15,946 & 22,470 & 1.41 & 88,097 & 117,703 & 1.34 & 6,589 & 8,114 & 1.23 \\
\hline
\end{tabular}


Table 1. Precipitation, irrigation water use, acres irrigated, irrigation application rate by system type, and soil permeability for counties in the Groundwater Management Districts within the Kansas High Plains, 1991-2003.-Continued

[Precipitation data from Kansas State Climatologist (2005). Irrigation data from Kansas Water Office and Kansas Department of Agriculture (1993-2005). Soil permeability from U.S. Department of Agriculture (1994). Blue shading indicates trend is significant at 95 -percent confidence level ( $\mathrm{p} \leq 0.05$ ). Green shading indicates trend is significant at 90 -percent confidence level ( $\mathrm{p} \leq 0.10)$. acre-ft, acre-feet; acreft/acre, acre-feet per acre; NA, data not available; --, not computed]

\begin{tabular}{|c|c|c|c|c|c|c|c|c|c|c|c|c|c|c|c|c|}
\hline \multirow[b]{2}{*}{ Year } & \multirow[b]{2}{*}{$\begin{array}{l}\text { Annual } \\
\text { precip- } \\
\text { itation } \\
\text { (inches) }\end{array}$} & \multirow{2}{*}{$\begin{array}{l}\text { March- } \\
\text { October } \\
\text { precipi- } \\
\text { tation } \\
\text { (inches) }\end{array}$} & \multirow[b]{2}{*}{$\begin{array}{c}\text { Total } \\
\text { irrigation } \\
\text { water use } \\
\text { (acre-ft) }\end{array}$} & \multirow[b]{2}{*}{$\begin{array}{c}\text { Total acres } \\
\text { irrigated }\end{array}$} & \multicolumn{3}{|c|}{ Flood irrigation } & \multicolumn{3}{|c|}{ Center pivot irrigation } & \multicolumn{3}{|c|}{$\begin{array}{l}\text { Center pivot irrigation with } \\
\text { drop nozzle }\end{array}$} & \multicolumn{3}{|c|}{ Other irrigation systems } \\
\hline & & & & & $\begin{array}{c}\text { Total } \\
\text { acres } \\
\text { irrigated }\end{array}$ & $\begin{array}{c}\text { Water } \\
\text { used } \\
\text { (acre-ft) }\end{array}$ & $\begin{array}{l}\text { Rate of } \\
\text { irri- } \\
\text { gation } \\
\text { (acre- } \\
\text { ft/acre) }\end{array}$ & $\begin{array}{c}\text { Total } \\
\text { acres } \\
\text { irrigated }\end{array}$ & $\begin{array}{c}\text { Water } \\
\text { used } \\
\text { (acre-ft) }\end{array}$ & $\begin{array}{l}\text { Rate of } \\
\text { irri- } \\
\text { gation } \\
\text { (acre- } \\
\text { ft/acre) }\end{array}$ & $\begin{array}{c}\text { Total } \\
\text { acres } \\
\text { irrigated }\end{array}$ & $\begin{array}{c}\text { Water } \\
\text { used } \\
\text { (acre-ft) }\end{array}$ & $\begin{array}{l}\text { Rate of } \\
\text { irri- } \\
\text { gation } \\
\text { (acre- } \\
\text { ft/acre) }\end{array}$ & $\begin{array}{c}\text { Total } \\
\text { acres } \\
\text { irrigated }\end{array}$ & $\begin{array}{c}\text { Water } \\
\text { used } \\
\text { (acre-ft) }\end{array}$ & $\begin{array}{c}\text { Rate of } \\
\text { irri- } \\
\text { gation } \\
\text { (acre- } \\
\text { ft/acre) }\end{array}$ \\
\hline \multicolumn{17}{|c|}{ Sherman County (estimated mean soil permeability $=1.3$ inches per hour) -Continued } \\
\hline 2003 & 14.70 & 13.98 & 144,636 & 116,603 & 4,182 & 5,762 & 1.38 & 14,268 & 17,991 & 1.26 & 91,727 & 113,302 & 1.24 & 6,426 & 7,582 & 1.18 \\
\hline Average & 19.02 & 17.31 & 120,794 & 111,857 & 17,737 & 22,541 & 1.28 & 39,898 & 38,594 & 1.03 & 51,000 & 57,025 & 1.00 & 7,146 & 7,021 & .99 \\
\hline p-value & 0 & -- & .02 & .02 & -- & -- & -- & -- & -- & -- & -- & -- & -- & -- & -- & -- \\
\hline slope & -.69 & -- & 4,699 & 1,054 & -- & -- & -- & -- & -- & -- & -- & -- & -- & -- & -- & -- \\
\hline \multicolumn{17}{|c|}{ Stafford County (estimated mean soil permeability $=6.3$ inches per hour) } \\
\hline 1991 & 15.25 & 11.83 & 108,602 & 78,445 & 5,975 & 8,604 & 1.44 & 69,563 & 96,693 & 1.39 & NA & NA & NA & 2,907 & 3,305 & 1.14 \\
\hline 1992 & 31.47 & 24.60 & 62,469 & 77,325 & 5,383 & 4,199 & .78 & 65,646 & 53,830 & .82 & 4,134 & 3,473 & .84 & 2,162 & 968 & .45 \\
\hline 1993 & 38.73 & 32.67 & 50,492 & 76,489 & 5,103 & 3,776 & .74 & 67,871 & 44,795 & .66 & 1,212 & 582 & .48 & 2,303 & 1,339 & .58 \\
\hline 1994 & 16.99 & 13.92 & 106,461 & 79,224 & 5,164 & 7,694 & 1.49 & 68,916 & 93,037 & 1.35 & 2,270 & 3,201 & 1.41 & 2,874 & 2,530 & .88 \\
\hline 1995 & 32.04 & 29.65 & 82,086 & 76,641 & 4,608 & 5,622 & 1.22 & 68,761 & 73,574 & 1.07 & 1,902 & 1,617 & .85 & 1,370 & 1,273 & .93 \\
\hline 1996 & 26.49 & 23.80 & 71,546 & 79,422 & 4,738 & 4,359 & .92 & 71,300 & 64,883 & .91 & 1,781 & 1,389 & .78 & 1,603 & 915 & .57 \\
\hline 1997 & 32.62 & 26.74 & 62,052 & 80,183 & 4,450 & 3,204 & .72 & 54,868 & 43,346 & .79 & 19,413 & 14,948 & .77 & 1,452 & 554 & .38 \\
\hline 1998 & 27.99 & 21.66 & 89,716 & 81,781 & 3,760 & 4,286 & 1.14 & 44,075 & 47,601 & 1.08 & 31,638 & 35,751 & 1.13 & 2,308 & 2,077 & .90 \\
\hline 1999 & 29.86 & 26.10 & 80,130 & 82,256 & 3,540 & 3,469 & .98 & 39,123 & 38,341 & .98 & 36,855 & 36,486 & .99 & 2,738 & 1,834 & .67 \\
\hline 2000 & 29.87 & 24.81 & 94,055 & 81,946 & 3,521 & 3,767 & 1.07 & 33,797 & 38,867 & 1.15 & 42,287 & 49,053 & 1.16 & 2,341 & 2,368 & 1.01 \\
\hline 2001 & 25.52 & 21.36 & 100,190 & 82,397 & 3,582 & 4,155 & 1.16 & 28,050 & 33,941 & 1.21 & 48,613 & 59,794 & 1.23 & 2,152 & 2,301 & 1.07 \\
\hline 2002 & 30.06 & 27.07 & 102,565 & 82,670 & 3,142 & 4,053 & 1.29 & 20,838 & 25,422 & 1.22 & 56,001 & 70,561 & 1.26 & 2,689 & 2,528 & .94 \\
\hline 2003 & 23.82 & 21.51 & 104,854 & 80,950 & 2,986 & 3,942 & 1.32 & 17,674 & 22,799 & 1.29 & 57,548 & 74,812 & 1.30 & 2,742 & 3,300 & 1.20 \\
\hline Average & 27.75 & 23.52 & 85,786 & 79,979 & 4,304 & 4,702 & 1.10 & 50,037 & 52,087 & 1.07 & 25,305 & 29,306 & 1.02 & 2,280 & 1,946 & .85 \\
\hline p-value & .85 & -- & .20 & 0 & -- & -- & -- & -- & -- & -- & -- & -- & -- & -- & -- & -- \\
\hline slope & -.20 & -- & 2,908 & 475 & -- & -- & -- & -- & -- & -- & -- & -- & -- & -- & -- & -- \\
\hline \multicolumn{17}{|c|}{ Stanton County (estimated mean soil permeability = 1.8 inches per hour) } \\
\hline 1991 & 18.22 & 15.20 & 236,953 & 151,971 & 92,302 & 148,606 & 1.61 & 32,928 & 47,416 & 1.44 & NA & NA & NA & 26,741 & 40,930 & 1.53 \\
\hline 1992 & 15.37 & 12.46 & 221,141 & 151,103 & 88,751 & 134,902 & 1.52 & 28,737 & 41,669 & 1.45 & 2,056 & 2,262 & 1.10 & 31,559 & 42,309 & 1.34 \\
\hline 1993 & 20.97 & 17.46 & 179,287 & 143,334 & 68,417 & 94,415 & 1.38 & 33,269 & 38,925 & 1.17 & 4,895 & 4,944 & 1.01 & 36,753 & 41,003 & 1.12 \\
\hline 1994 & 17.04 & 15.49 & 198,112 & 140,109 & 51,219 & 77,853 & 1.52 & 31,465 & 39,961 & 1.27 & 16,858 & 24,613 & 1.46 & 40,567 & 55,686 & 1.37 \\
\hline 1995 & 15.46 & 14.69 & 182,745 & 134,596 & 33,602 & 50,403 & 1.50 & 38,762 & 48,453 & 1.25 & 14,003 & 22,265 & 1.59 & 48,229 & 61,624 & 1.28 \\
\hline 1996 & 23.91 & 22.85 & 156,832 & 130,683 & 32,998 & 42,567 & 1.29 & 36,219 & 39,117 & 1.08 & 18,296 & 23,968 & 1.31 & 43,170 & 51,180 & 1.19 \\
\hline
\end{tabular}


Table 1. Precipitation, irrigation water use, acres irrigated, irrigation application rate by system type, and soil permeability for counties in the Groundwater Management Districts within the Kansas High Plains, 1991-2003.-Continued

[Precipitation data from Kansas State Climatologist (2005). Irrigation data from Kansas Water Office and Kansas Department of Agriculture (1993-2005). Soil permeability from U.S. Department of Agriculture (1994). Blue shading indicates trend is significant at 95-percent confidence level ( $\mathrm{p} \leq 0.05)$. Green shading indicates trend is significant at 90-percent confidence level ( $\mathrm{p} \leq 0.10)$. acre-ft, acre-feet; acre$\mathrm{ft} / \mathrm{acre}$, acre-feet per acre; NA, data not available; --, not computed]

\begin{tabular}{|c|c|c|c|c|c|c|c|c|c|c|c|c|c|c|c|c|}
\hline \multirow[b]{2}{*}{ Year } & \multirow[b]{2}{*}{$\begin{array}{l}\text { Annual } \\
\text { precip- } \\
\text { itation } \\
\text { (inches) }\end{array}$} & \multirow[b]{2}{*}{$\begin{array}{l}\text { March- } \\
\text { October } \\
\text { precipi- } \\
\text { tation } \\
\text { (inches) }\end{array}$} & \multirow[b]{2}{*}{$\begin{array}{c}\text { Total } \\
\text { irrigation } \\
\text { water use } \\
\text { (acre-ft) }\end{array}$} & \multirow[b]{2}{*}{$\begin{array}{c}\text { Total acres } \\
\text { irrigated }\end{array}$} & \multicolumn{3}{|c|}{ Flood irrigation } & \multicolumn{3}{|c|}{ Center pivot irrigation } & \multicolumn{3}{|c|}{$\begin{array}{l}\text { Center pivot irrigation with } \\
\text { drop nozzle }\end{array}$} & \multicolumn{3}{|c|}{ Other irrigation systems } \\
\hline & & & & & $\begin{array}{c}\text { Total } \\
\text { acres } \\
\text { irrigated }\end{array}$ & $\begin{array}{c}\text { Water } \\
\text { used } \\
\text { (acre-ft) }\end{array}$ & $\begin{array}{c}\text { Rate of } \\
\text { irri- } \\
\text { gation } \\
\text { (acre- } \\
\text { ft/acre) }\end{array}$ & $\begin{array}{c}\text { Total } \\
\text { acres } \\
\text { irrigated }\end{array}$ & $\begin{array}{c}\text { Water } \\
\text { used } \\
\text { (acre-ft) }\end{array}$ & $\begin{array}{l}\text { Rate of } \\
\text { irri- } \\
\text { gation } \\
\text { (acre- } \\
\text { ft/acre) }\end{array}$ & $\begin{array}{c}\text { Total } \\
\text { acres } \\
\text { irrigated }\end{array}$ & $\begin{array}{c}\text { Water } \\
\text { used } \\
\text { (acre-ft) }\end{array}$ & $\begin{array}{l}\text { Rate of } \\
\text { irri- } \\
\text { gation } \\
\text { (acre- } \\
\text { ft/acre) }\end{array}$ & $\begin{array}{c}\text { Total } \\
\text { acres } \\
\text { irrigated }\end{array}$ & $\begin{array}{c}\text { Water } \\
\text { used } \\
\text { (acre-ft) }\end{array}$ & $\begin{array}{l}\text { Rate of } \\
\text { irri- } \\
\text { gation } \\
\text { (acre- } \\
\text { ft/acre) }\end{array}$ \\
\hline \multicolumn{17}{|c|}{ Stanton County (estimated mean soil permeability $=1.8$ inches per hour) —Continued } \\
\hline 1997 & 19.72 & 17.90 & 145,945 & 135,102 & 25,567 & 31,959 & 1.25 & 29,475 & 28,886 & 0.98 & 32,000 & 35,520 & 1.11 & 48,060 & 49,581 & 1.03 \\
\hline 1998 & 19.12 & 17.03 & 140,609 & 134,864 & 16,699 & 19,538 & 1.17 & 32,177 & 29,925 & .93 & 42,147 & 43,833 & 1.04 & 43,841 & 47,314 & 1.08 \\
\hline 1999 & 16.76 & 15.40 & 150,627 & 135,914 & 15,687 & 19,922 & 1.27 & 18,910 & 18,532 & .98 & 55,320 & 59,746 & 1.08 & 45,997 & 52,427 & 1.14 \\
\hline 2000 & 17.52 & 16.90 & 167,664 & 132,508 & 10,154 & 15,028 & 1.48 & 17,956 & 19,752 & 1.10 & 61,813 & 77,884 & 1.26 & 42,585 & 55,000 & 1.29 \\
\hline 2001 & 19.54 & 17.17 & 125,333 & 127,360 & 6,235 & 7,295 & 1.17 & 14,520 & 13,213 & .91 & 60,539 & 58,117 & .96 & 46,066 & 46,707 & 1.01 \\
\hline 2002 & 12.87 & 11.24 & 161,130 & 141,815 & 7,935 & 8,252 & 1.04 & 8,339 & 9,673 & 1.16 & 71,754 & 91,128 & 1.27 & 53,787 & 52,077 & .97 \\
\hline 2003 & 15.53 & 13.84 & 138,028 & 116,663 & 3,708 & 5,636 & 1.52 & 12,304 & 11,689 & .95 & 63,049 & 75,659 & 1.20 & 37,602 & 45,044 & 1.20 \\
\hline Average & 17.85 & 15.97 & 169,570 & 136,617 & 34,867 & 50,491 & 1.36 & 25,774 & 29,785 & 1.13 & 36,894 & 43,328 & 1.20 & 41,920 & 49,299 & 1.18 \\
\hline p-value & .50 & -- & 0 & .01 & -- & -- & -- & -- & -- & -- & -- & -- & -- & -- & -- & -- \\
\hline slope & -.20 & -- & $-7,520$ & $-2,032$ & -- & -- & -- & -- & -- & -- & -- & -- & -- & -- & -- & -- \\
\hline \multicolumn{17}{|c|}{ Stevens County (estimated mean soil permeability = 3.1 inches per hour) } \\
\hline 1991 & 23.33 & 19.47 & 235,817 & 137,515 & 47,441 & 87,291 & 1.84 & 62,747 & 105,415 & 1.68 & NA & NA & NA & 27,327 & 43,110 & 1.58 \\
\hline 1992 & 14.85 & 11.48 & 225,734 & 140,364 & 41,411 & 74,540 & 1.80 & 55,380 & 89,716 & 1.62 & 6,812 & 8,106 & 1.19 & 36,761 & 53,372 & 1.45 \\
\hline 1993 & 20.63 & 16.73 & 180,710 & 136,350 & 28,177 & 40,293 & 1.43 & 59,007 & 78,479 & 1.33 & 8,938 & 8,759 & .98 & 40,228 & 53,178 & 1.32 \\
\hline 1994 & 20.96 & 19.21 & 206,797 & 137,627 & 24,331 & 41,849 & 1.72 & 63,455 & 95,183 & 1.50 & 9,259 & 10,926 & 1.18 & 40,582 & 58,840 & 1.45 \\
\hline 1995 & 13.04 & 12.01 & 209,056 & 140,006 & 21,394 & 36,798 & 1.72 & 70,212 & 101,807 & 1.45 & 7,600 & 9,956 & 1.31 & 40,800 & 60,495 & 1.48 \\
\hline 1996 & 21.53 & 20.67 & 212,109 & 141,371 & 21,905 & 33,953 & 1.55 & 74,093 & 111,140 & 1.50 & 12,065 & 16,046 & 1.33 & 33,308 & 50,970 & 1.53 \\
\hline 1997 & 20.66 & 17.74 & 207,239 & 156,542 & 16,252 & 25,678 & 1.58 & 66,646 & 87,973 & 1.32 & 39,026 & 48,783 & 1.25 & 34,618 & 44,805 & 1.29 \\
\hline 1998 & 15.38 & 14.56 & 217,128 & 161,139 & 10,669 & 14,723 & 1.38 & 60,906 & 78,569 & 1.29 & 48,544 & 66,505 & 1.37 & 41,020 & 57,331 & 1.40 \\
\hline 1999 & 19.47 & 17.83 & 197,888 & 169,084 & 8,550 & 11,457 & 1.34 & 50,380 & 56,929 & 1.13 & 73,410 & 88,092 & 1.20 & 36,744 & 41,410 & 1.13 \\
\hline 2000 & 17.04 & 16.85 & 236,647 & 171,907 & 7,962 & 11,784 & 1.48 & 40,350 & 52,859 & 1.31 & 91,783 & 130,332 & 1.42 & 31,812 & 41,673 & 1.31 \\
\hline 2001 & 15.04 & 12.42 & 210,617 & 168,675 & 6,018 & 7,161 & 1.19 & 32,731 & 40,259 & 1.23 & 103,016 & 131,860 & 1.28 & 26,910 & 31,336 & 1.16 \\
\hline 2002 & 12.87 & 11.04 & 282,752 & 172,069 & 3,093 & 6,124 & 1.98 & 27,788 & 43,905 & 1.58 & 106,621 & 176,991 & 1.66 & 34,567 & 55,732 & 1.61 \\
\hline 2003 & 24.81 & 23.76 & 203,187 & 171,049 & 3,461 & 4,603 & 1.33 & 22,124 & 26,991 & 1.22 & 119,410 & 139,710 & 1.17 & 26,054 & 31,883 & 1.22 \\
\hline Average & 18.43 & 16.44 & 217,360 & 154,131 & 18,513 & 30,481 & 1.56 & 52,755 & 74,556 & 1.40 & 52,207 & 69,672 & 1.28 & 34,672 & 48,010 & 1.38 \\
\hline p-value & .43 & -- & .85 & 0 & -- & -- & -- & -- & -- & -- & -- & -- & -- & -- & -- & -- \\
\hline slope & -.40 & -- & 203 & 3,384 & -- & -- & -- & -- & -- & -- & -- & -- & -- & -- & -- & -- \\
\hline
\end{tabular}


Table 1. Precipitation, irrigation water use, acres irrigated, irrigation application rate by system type, and soil permeability for counties in the Groundwater Management Districts within the Kansas High Plains, 1991-2003.-Continued

[Precipitation data from Kansas State Climatologist (2005). Irrigation data from Kansas Water Office and Kansas Department of Agriculture (1993-2005). Soil permeability from U.S. Department of Agriculture (1994). Blue shading indicates trend is significant at 95 -percent confidence level ( $\mathrm{p} \leq 0.05$ ). Green shading indicates trend is significant at 90 -percent confidence level ( $\mathrm{p} \leq 0.10)$. acre-ft, acre-feet; acreft/acre, acre-feet per acre; NA, data not available; --, not computed]

\begin{tabular}{|c|c|c|c|c|c|c|c|c|c|c|c|c|c|c|c|c|}
\hline \multirow[b]{2}{*}{ Year } & \multirow[b]{2}{*}{$\begin{array}{l}\text { Annual } \\
\text { precip- } \\
\text { itation } \\
\text { (inches) }\end{array}$} & \multirow{2}{*}{$\begin{array}{l}\text { March- } \\
\text { October } \\
\text { precipi- } \\
\text { tation } \\
\text { (inches) }\end{array}$} & \multirow[b]{2}{*}{$\begin{array}{c}\text { Total } \\
\text { irrigation } \\
\text { water use } \\
\text { (acre-ft) }\end{array}$} & \multirow[b]{2}{*}{$\begin{array}{c}\text { Total acres } \\
\text { irrigated }\end{array}$} & \multicolumn{3}{|c|}{ Flood irrigation } & \multicolumn{3}{|c|}{ Center pivot irrigation } & \multicolumn{3}{|c|}{$\begin{array}{l}\text { Center pivot irrigation with } \\
\text { drop nozzle }\end{array}$} & \multicolumn{3}{|c|}{ Other irrigation systems } \\
\hline & & & & & $\begin{array}{c}\text { Total } \\
\text { acres } \\
\text { irrigated }\end{array}$ & $\begin{array}{c}\text { Water } \\
\text { used } \\
\text { (acre-ft) }\end{array}$ & $\begin{array}{l}\text { Rate of } \\
\text { irri- } \\
\text { gation } \\
\text { (acre- } \\
\text { ft/acre) }\end{array}$ & $\begin{array}{c}\text { Total } \\
\text { acres } \\
\text { irrigated }\end{array}$ & $\begin{array}{c}\text { Water } \\
\text { used } \\
\text { (acre-ft) }\end{array}$ & $\begin{array}{l}\text { Rate of } \\
\text { irri- } \\
\text { gation } \\
\text { (acre- } \\
\text { ft/acre) }\end{array}$ & $\begin{array}{c}\text { Total } \\
\text { acres } \\
\text { irrigated }\end{array}$ & $\begin{array}{c}\text { Water } \\
\text { used } \\
\text { (acre-ft) }\end{array}$ & $\begin{array}{l}\text { Rate of } \\
\text { irri- } \\
\text { gation } \\
\text { (acre- } \\
\text { ft/acre) }\end{array}$ & $\begin{array}{c}\text { Total } \\
\text { acres } \\
\text { irrigated }\end{array}$ & $\begin{array}{c}\text { Water } \\
\text { used } \\
\text { (acre-ft) }\end{array}$ & $\begin{array}{c}\text { Rate of } \\
\text { irri- } \\
\text { gation } \\
\text { (acre- } \\
\text { ft/acre) }\end{array}$ \\
\hline \multicolumn{17}{|c|}{ Thomas County (estimated mean soil permeability = 1.3 inches per hour) } \\
\hline 1991 & 20.98 & 18.14 & 132,056 & 97,627 & 15,297 & 23,404 & 1.53 & 77,124 & 101,032 & 1.31 & NA & NA & NA & 5,206 & 7,619 & 1.46 \\
\hline 1992 & 25.57 & 22.38 & 62,986 & 92,800 & 9,821 & 8,151 & .83 & 62,032 & 40,321 & .65 & 15,895 & 10,650 & .67 & 5,052 & 3,864 & .76 \\
\hline 1993 & 29.74 & 26.23 & 71,509 & 95,163 & 9,346 & 9,066 & .97 & 57,393 & 44,193 & .77 & 23,332 & 14,699 & .63 & 5,092 & 3,551 & .70 \\
\hline 1994 & 22.36 & 19.35 & 107,334 & 98,737 & 9,266 & 11,583 & 1.25 & 55,464 & 62,120 & 1.12 & 26,072 & 26,072 & 1.00 & 7,935 & 7,560 & .95 \\
\hline 1995 & 21.80 & 20.90 & 98,976 & 95,327 & 6,850 & 7,946 & 1.16 & 58,488 & 62,582 & 1.07 & 26,624 & 24,760 & .93 & 3,365 & 3,687 & 1.10 \\
\hline 1996 & 21.61 & 20.88 & 98,411 & 98,853 & 5,546 & 6,378 & 1.15 & 59,155 & 59,155 & 1.00 & 28,970 & 27,811 & .96 & 5,182 & 5,067 & .98 \\
\hline 1997 & 20.97 & 18.52 & 109,941 & 99,975 & 5,227 & 6,272 & 1.20 & 37,263 & 40,989 & 1.10 & 51,926 & 56,080 & 1.08 & 5,559 & 6,599 & 1.19 \\
\hline 1998 & 22.45 & 18.91 & 98,810 & 98,595 & 2,877 & 3,050 & 1.06 & 27,458 & 28,007 & 1.02 & 63,572 & 62,936 & .99 & 4,688 & 4,817 & 1.03 \\
\hline 1999 & 20.64 & 19.95 & 82,629 & 99,190 & 2,097 & 1,950 & .93 & 24,760 & 21,294 & .86 & 67,169 & 55,079 & .82 & 5,164 & 4,306 & .83 \\
\hline 2000 & 16.32 & 14.35 & 133,946 & 101,694 & 1,721 & 2,513 & 1.46 & 19,756 & 26,473 & 1.34 & 73,269 & 96,715 & 1.32 & 6,948 & 8,245 & 1.19 \\
\hline 2001 & 21.23 & 18.16 & 110,032 & 99,864 & 1,521 & 1,856 & 1.22 & 13,674 & 15,862 & 1.16 & 79,062 & 86,178 & 1.09 & 5,607 & 6,137 & 1.09 \\
\hline 2002 & 12.69 & 12.19 & 135,487 & 101,526 & 1,285 & 1,953 & 1.52 & 11,547 & 14,780 & 1.28 & 82,751 & 110,886 & 1.34 & 5,943 & 7,868 & 1.32 \\
\hline 2003 & 14.00 & 13.32 & 121,408 & 101,552 & 600 & 912 & 1.52 & 11,353 & 12,602 & 1.11 & 83,070 & 99,684 & 1.20 & 6,529 & 8,210 & 1.26 \\
\hline Average & 20.80 & 18.71 & 104,887 & 98,531 & 5,496 & 6,541 & 1.22 & 39,651 & 40,724 & 1.06 & 51,809 & 55,963 & 1.00 & 5,559 & 5,964 & 1.07 \\
\hline p-value & .01 & -- & .08 & 0 & -- & -- & -- & -- & -- & -- & -- & -- & -- & -- & -- & -- \\
\hline slope & -.85 & -- & 3,402 & 580 & -- & -- & -- & -- & -- & -- & -- & -- & -- & -- & -- & -- \\
\hline \multicolumn{17}{|c|}{ Wallace County (estimated mean soil permeability = 1.4 inches per hour) } \\
\hline 1991 & 21.05 & 18.03 & 87,142 & 60,361 & 32,528 & 53,671 & 1.65 & 22,906 & 25,426 & 1.11 & NA & NA & NA & 4,927 & 8,045 & 1.63 \\
\hline 1992 & 21.10 & 17.54 & 67,807 & 56,743 & 27,293 & 37,664 & 1.38 & 19,577 & 20,947 & 1.07 & 6,443 & 5,541 & .86 & 3,430 & 3,654 & 1.07 \\
\hline 1993 & 25.39 & 21.58 & 54,381 & 58,275 & 22,241 & 25,355 & 1.14 & 17,631 & 14,986 & .85 & 11,897 & 8,447 & .71 & 6,506 & 5,593 & .86 \\
\hline 1994 & 16.75 & 14.11 & 75,377 & 59,008 & 18,654 & 28,168 & 1.51 & 21,122 & 24,290 & 1.15 & 10,218 & 11,035 & 1.08 & 9,014 & 11,884 & 1.32 \\
\hline 1995 & 22.37 & 21.22 & 68,972 & 57,233 & 16,133 & 25,167 & 1.56 & 21,451 & 21,022 & .98 & 11,692 & 12,510 & 1.07 & 7,957 & 10,272 & 1.29 \\
\hline 1996 & 18.87 & 18.23 & 80,443 & 61,595 & 17,533 & 30,157 & 1.72 & 19,540 & 21,494 & 1.10 & 16,733 & 18,406 & 1.10 & 7,789 & 10,386 & 1.33 \\
\hline 1997 & 22.38 & 19.31 & 64,362 & 60,573 & 13,730 & 19,634 & 1.43 & 14,762 & 13,138 & .89 & 23,696 & 22,037 & .93 & 8,385 & 9,553 & 1.14 \\
\hline 1998 & 18.09 & 15.63 & 66,462 & 60,573 & 10,491 & 13,428 & 1.28 & 12,587 & 12,209 & .97 & 30,255 & 31,465 & 1.04 & 7,240 & 9,358 & 1.29 \\
\hline 1999 & 24.81 & 23.88 & 59,611 & 60,660 & 7,772 & 9,326 & 1.20 & 11,834 & 9,941 & .84 & 33,007 & 31,027 & .94 & 8,047 & 9,317 & 1.16 \\
\hline 2000 & 19.85 & 17.39 & 81,560 & 60,832 & 6,801 & 11,222 & 1.65 & 7,630 & 7,630 & 1.00 & 38,095 & 50,285 & 1.32 & 8,306 & 12,423 & 1.50 \\
\hline 2001 & 15.98 & 13.15 & 70,014 & 59,998 & 5,740 & 8,954 & 1.56 & 8,270 & 8,187 & .99 & 38,008 & 42,949 & 1.13 & 7,980 & 9,923 & 1.24 \\
\hline
\end{tabular}


Table 1. Precipitation, irrigation water use, acres irrigated, irrigation application rate by system type, and soil permeability for counties in the Groundwater Management Districts within the Kansas High Plains, 1991-2003.-Continued

[Precipitation data from Kansas State Climatologist (2005). Irrigation data from Kansas Water Office and Kansas Department of Agriculture (1993-2005). Soil permeability from U.S. Department of Agriculture (1994). Blue shading indicates trend is significant at 95-percent confidence level ( $\mathrm{p} \leq 0.05$ ). Green shading indicates trend is significant at 90 -percent confidence level ( $\mathrm{p} \leq 0.10)$. acre-ft, acre-feet; acreft/acre, acre-feet per acre; NA, data not available; --, not computed]

\begin{tabular}{|c|c|c|c|c|c|c|c|c|c|c|c|c|c|c|c|c|}
\hline \multirow[b]{2}{*}{ Year } & \multirow[b]{2}{*}{$\begin{array}{l}\text { Annual } \\
\text { precip- } \\
\text { itation } \\
\text { (inches) }\end{array}$} & \multirow{2}{*}{$\begin{array}{l}\text { March- } \\
\text { October } \\
\text { precipi- } \\
\text { tation } \\
\text { (inches) }\end{array}$} & \multirow[b]{2}{*}{$\begin{array}{c}\text { Total } \\
\text { irrigation } \\
\text { water use } \\
\text { (acre-ft) }\end{array}$} & \multirow[b]{2}{*}{$\begin{array}{c}\text { Total acres } \\
\text { irrigated }\end{array}$} & \multicolumn{3}{|c|}{ Flood irrigation } & \multicolumn{3}{|c|}{ Center pivot irrigation } & \multicolumn{3}{|c|}{$\begin{array}{l}\text { Center pivot irrigation with } \\
\text { drop nozzle }\end{array}$} & \multicolumn{3}{|c|}{ Other irrigation systems } \\
\hline & & & & & $\begin{array}{c}\text { Total } \\
\text { acres } \\
\text { irrigated }\end{array}$ & $\begin{array}{c}\text { Water } \\
\text { used } \\
\text { (acre-ft) }\end{array}$ & $\begin{array}{l}\text { Rate of } \\
\text { irri- } \\
\text { gation } \\
\text { (acre- } \\
\text { ft/acre) }\end{array}$ & $\begin{array}{c}\text { Total } \\
\text { acres } \\
\text { irrigated }\end{array}$ & $\begin{array}{c}\text { Water } \\
\text { used } \\
\text { (acre-ft) }\end{array}$ & $\begin{array}{l}\text { Rate of } \\
\text { irri- } \\
\text { gation } \\
\text { (acre- } \\
\text { ft/acre) }\end{array}$ & $\begin{array}{c}\text { Total } \\
\text { acres } \\
\text { irrigated }\end{array}$ & $\begin{array}{c}\text { Water } \\
\text { used } \\
\text { (acre-ft) }\end{array}$ & $\begin{array}{l}\text { Rate of } \\
\text { irri- } \\
\text { gation } \\
\text { (acre- } \\
\text { ft/acre) }\end{array}$ & $\begin{array}{c}\text { Total } \\
\text { acres } \\
\text { irrigated }\end{array}$ & $\begin{array}{c}\text { Water } \\
\text { used } \\
\text { (acre-ft) }\end{array}$ & $\begin{array}{l}\text { Rate of } \\
\text { irri- } \\
\text { gation } \\
\text { (acre- } \\
\mathrm{ft} / \text { acre) }\end{array}$ \\
\hline \multicolumn{17}{|c|}{ Wallace County (estimated mean soil permeability $=1.4$ inches per hour) —Continued } \\
\hline 2002 & 13.49 & 12.28 & 84,891 & 59,227 & 4,784 & 8,563 & 1.79 & 5,916 & 6,981 & 1.18 & 37,957 & 53,140 & 1.40 & 10,570 & 16,207 & 1.53 \\
\hline 2003 & 18.63 & 16.94 & 65,132 & 56,883 & 3,374 & 4,859 & 1.44 & 6,654 & 6,388 & .96 & 39,859 & 45,041 & 1.13 & 6,996 & 8,845 & 1.26 \\
\hline Average & 19.90 & 17.64 & 71,243 & 59,382 & 14,390 & 21,244 & 1.49 & 14,606 & 14,818 & 1.01 & 24,822 & 27,657 & 1.06 & 7,473 & 9,651 & 1.29 \\
\hline p-value & .16 & -- & 1.0 & .62 & -- & -- & -- & -- & -- & -- & -- & -- & -- & -- & -- & -- \\
\hline slope & -.45 & -- & -38 & 36 & -- & -- & -- & -- & -- & -- & -- & -- & -- & -- & -- & -- \\
\hline \multicolumn{17}{|c|}{ Wichita County (estimated mean soil permeability $=1.2$ inches per hour) } \\
\hline 1991 & 15.58 & 11.95 & 119,283 & 90,211 & 84,379 & 113,068 & 1.34 & 3,894 & 3,855 & 0.99 & NA & NA & NA & 1,938 & 2,360 & 1.22 \\
\hline 1992 & 23.35 & 18.77 & 89,822 & 84,679 & 76,173 & 82,267 & 1.08 & 2,884 & 2,769 & .96 & 353 & 124 & .35 & 5,269 & 4,663 & .89 \\
\hline 1993 & 27.10 & 22.44 & 62,140 & 74,155 & 61,279 & 53,313 & .87 & 4,486 & 3,185 & .71 & 3,033 & 1,880 & .62 & 5,357 & 3,762 & .70 \\
\hline 1994 & 19.34 & 17.29 & 89,985 & 80,031 & 60,342 & 68,790 & 1.14 & 5,696 & 6,095 & 1.07 & 8,174 & 8,746 & 1.07 & 5,819 & 6,355 & 1.09 \\
\hline 1995 & 19.17 & 18.42 & 81,526 & 80,759 & 53,672 & 56,892 & 1.06 & 9,933 & 9,039 & .91 & 7,221 & 7,221 & 1.00 & 9,933 & 8,374 & .84 \\
\hline 1996 & 19.13 & 17.75 & 78,341 & 79,777 & 47,274 & 48,692 & 1.03 & 14,353 & 12,631 & .88 & 10,340 & 8,582 & .83 & 7,810 & 8,436 & 1.08 \\
\hline 1997 & 27.02 & 23.05 & 71,603 & 82,188 & 38,545 & 37,774 & .98 & 15,922 & 11,145 & .70 & 21,541 & 17,017 & .79 & 6,180 & 5,666 & .92 \\
\hline 1998 & 18.96 & 15.85 & 68,012 & 77,496 & 30,928 & 28,454 & .92 & 15,205 & 11,100 & .73 & 22,943 & 22,255 & .97 & 8,420 & 6,204 & .74 \\
\hline 1999 & 21.64 & 21.05 & 70,294 & 82,218 & 30,178 & 28,066 & .93 & 12,602 & 9,830 & .78 & 31,751 & 25,718 & .81 & 7,687 & 6,681 & .87 \\
\hline 2000 & 20.62 & 18.85 & 86,257 & 83,347 & 26,326 & 28,169 & 1.07 & 11,686 & 10,050 & .86 & 36,444 & 37,537 & 1.03 & 8,891 & 10,501 & 1.18 \\
\hline 2001 & 19.82 & 17.46 & 62,082 & 75,350 & 19,986 & 17,788 & .89 & 11,185 & 8,277 & .74 & 36,345 & 28,713 & .79 & 7,834 & 7,305 & .93 \\
\hline 2002 & 15.62 & 15.05 & 86,350 & 81,497 & 22,564 & 24,369 & 1.08 & 9,379 & 8,441 & .90 & 36,845 & 39,793 & 1.08 & 12,709 & 13,747 & 1.08 \\
\hline 2003 & 20.04 & 18.69 & 64,063 & 75,681 & 18,136 & 16,322 & .90 & 5,593 & 3,580 & .64 & 41,887 & 36,023 & .86 & 10,065 & 8,138 & .81 \\
\hline Average & 20.57 & 18.20 & 79,212 & 80,568 & 43,829 & 46,459 & 1.02 & 9,448 & 7,692 & .84 & 21,406 & 19,467 & .85 & 7,532 & 7,092 & .94 \\
\hline p-value & .67 & -- & .08 & .36 & -- & -- & -- & -- & -- & -- & -- & -- & -- & -- & -- & -- \\
\hline slope & -.09 & -- & $-2,723$ & 491 & -- & -- & -- & -- & -- & -- & -- & -- & -- & -- & -- & -- \\
\hline
\end{tabular}


Table 2. Irrigation water use, acres irrigated, and irrigation application rate by crop type for counties in the Groundwater Management Districts within the Kansas High Plains,

[Data from Kansas Water Office and Kansas Department of Agriculture (1993-2005). acre-ft, acre-foot; acre-ft/acre, acre-foot per acre]

\begin{tabular}{|c|c|c|c|c|c|c|c|c|c|c|c|c|c|c|c|c|c|c|}
\hline \multirow[b]{2}{*}{ Year } & \multicolumn{3}{|c|}{ Alfalfa } & \multicolumn{3}{|c|}{ Corn } & \multicolumn{3}{|c|}{ Grain sorghum } & \multicolumn{3}{|c|}{ Soybeans } & \multicolumn{3}{|c|}{ Wheat } & \multicolumn{3}{|c|}{ Other/Multiple use } \\
\hline & $\begin{array}{c}\text { Total } \\
\text { acres } \\
\text { irrigated }\end{array}$ & $\begin{array}{c}\text { Irriga- } \\
\text { tion } \\
\text { water } \\
\text { used } \\
\text { (acre-ft) }\end{array}$ & $\begin{array}{c}\text { Rate of } \\
\text { irri- } \\
\text { gation } \\
\text { (acre- } \\
\text { ft/acre) }\end{array}$ & $\begin{array}{c}\text { Total } \\
\text { acres } \\
\text { irrigated }\end{array}$ & $\begin{array}{c}\text { Irriga- } \\
\text { tion } \\
\text { water } \\
\text { used } \\
\text { (acre-ft) }\end{array}$ & $\begin{array}{l}\text { Rate of } \\
\text { irri- } \\
\text { gation } \\
\text { (acre- } \\
\text { ft/acre) }\end{array}$ & $\begin{array}{c}\text { Total } \\
\text { acres } \\
\text { irrigated }\end{array}$ & $\begin{array}{c}\text { Irriga- } \\
\text { tion } \\
\text { water } \\
\text { used } \\
\text { (acre-ft) }\end{array}$ & $\begin{array}{l}\text { Rate of } \\
\text { irri- } \\
\text { gation } \\
\text { (acre- } \\
\text { ft/acre) }\end{array}$ & $\begin{array}{c}\text { Total } \\
\text { acres } \\
\text { irrigated }\end{array}$ & $\begin{array}{c}\text { Irriga- } \\
\text { tion } \\
\text { water } \\
\text { used } \\
\text { (acre-ft) }\end{array}$ & $\begin{array}{l}\text { Rate of } \\
\text { irri- } \\
\text { gation } \\
\text { (acre- } \\
\text { ft/acre) }\end{array}$ & $\begin{array}{c}\text { Total } \\
\text { acres } \\
\text { irrigated }\end{array}$ & $\begin{array}{c}\text { Irriga- } \\
\text { tion } \\
\text { water } \\
\text { used } \\
\text { (acre-ft) }\end{array}$ & $\begin{array}{l}\text { Rate of } \\
\text { irri- } \\
\text { gation } \\
\text { (acre- } \\
\mathrm{ft} / \text { acre) }\end{array}$ & $\begin{array}{c}\text { Total } \\
\text { acres } \\
\text { irrigated }\end{array}$ & $\begin{array}{c}\text { Irriga- } \\
\text { tion } \\
\text { water } \\
\text { used } \\
\text { (acre-ft) }\end{array}$ & $\begin{array}{c}\text { Rate of } \\
\text { irri- } \\
\text { gation } \\
\text { (acre- } \\
\text { ft/acre) }\end{array}$ \\
\hline \multicolumn{19}{|c|}{ Barton County } \\
\hline 1991 & 2,737 & 3,722 & 1.36 & 11,359 & 16,016 & 1.41 & 3,206 & 3,623 & 1.13 & 1,840 & 2,668 & 1.45 & 1,055 & 580 & 0.55 & 17,711 & 24,264 & 1.37 \\
\hline 1992 & 2,518 & 1,410 & .56 & 11,687 & 8,064 & .69 & 2,016 & 766 & .38 & 2,083 & 1,062 & .51 & 900 & 270 & .30 & 13,304 & 8,648 & .65 \\
\hline 1993 & 2,377 & 1,046 & .44 & 12,284 & 7,616 & .62 & 2,215 & 952 & .43 & 1,261 & 681 & .54 & 259 & 111 & .43 & 13,486 & 7,552 & .56 \\
\hline 1994 & 3,028 & 3,512 & 1.16 & 13,433 & 17,732 & 1.32 & 2,575 & 2,498 & .97 & 2,691 & 3,471 & 1.29 & 336 & 373 & 1.11 & 13,362 & 16,168 & 1.21 \\
\hline 1995 & 2,938 & 2,115 & .72 & 10,971 & 11,629 & 1.06 & 3,207 & 2,437 & .76 & 2,819 & 2,960 & 1.05 & 254 & 112 & .44 & 15,815 & 14,234 & .90 \\
\hline 1996 & 2,897 & 2,231 & .77 & 12,900 & 13,803 & 1.07 & 2,881 & 2,046 & .71 & 2,106 & 2,064 & .98 & 140 & 202 & 1.44 & 15,492 & 12,858 & .83 \\
\hline 1997 & 3,170 & 2,029 & .64 & 12,678 & 10,396 & .82 & 2,405 & 1,395 & .58 & 3,697 & 2,218 & .60 & 262 & 121 & .46 & 14,183 & 8,793 & .62 \\
\hline 1998 & 3,354 & 2,884 & .86 & 12,401 & 11,533 & .93 & 1,855 & 1,187 & .64 & 3,749 & 3,749 & 10 & 512 & 113 & .22 & 14,364 & 10,773 & .75 \\
\hline 1999 & 3,240 & 1,976 & .61 & 10,625 & 9,244 & .87 & 1,763 & 1,075 & .61 & 6,012 & 5,351 & .89 & 203 & 37 & .18 & 15,024 & 10,367 & .69 \\
\hline 2000 & 2,929 & 2,578 & .88 & 11,556 & 12,249 & 1.06 & 1,683 & 1,532 & .91 & 5,218 & 5,896 & 1.13 & 451 & 469 & 1.04 & 15,701 & 15,387 & .98 \\
\hline 2001 & 2,520 & 1,915 & .76 & 10,693 & 13,152 & 1.23 & 1,606 & 1,799 & 1.12 & 6,650 & 7,714 & 1.16 & 869 & 547 & .63 & 14,327 & 14,757 & 1.03 \\
\hline 2002 & 4,075 & 3,953 & .97 & 11,689 & 13,209 & 1.13 & 1,871 & 1,983 & 1.06 & 5,238 & 6,390 & 1.22 & 530 & 276 & .52 & 14,801 & 14,357 & .97 \\
\hline 2003 & 4,455 & 4,188 & .94 & 10,645 & 13,306 & 1.25 & 2,012 & 2,052 & 1.02 & 4,132 & 5,289 & 1.28 & 642 & 295 & .46 & 15,789 & 17,684 & 1.12 \\
\hline Average & 3,095 & 2,582 & .82 & 11,763 & 12,150 & 1.04 & 2,253 & 1,796 & .79 & 3,654 & 3,809 & 1.01 & 493 & 270 & .60 & 14,874 & 13,526 & .90 \\
\hline \multicolumn{19}{|c|}{ Cheyenne County } \\
\hline 1991 & 3,072 & 4,454 & 1.45 & 16,491 & 24,242 & 1.47 & 1,949 & 1,813 & .93 & 745 & 946 & 1.27 & 1,840 & 1,546 & .84 & 19,882 & 23,063 & 1.16 \\
\hline 1992 & 3,030 & 3,909 & 1.29 & 16,823 & 15,309 & .91 & 2,560 & 3,021 & 1.18 & 780 & 593 & .76 & 1,578 & 836 & .53 & 17,443 & 15,873 & .91 \\
\hline 1993 & 3,206 & 3,623 & 1.13 & 20,150 & 22,770 & 1.13 & 531 & 446 & .84 & 843 & 624 & .74 & 2,077 & 1,558 & .75 & 16,900 & 15,210 & .90 \\
\hline 1994 & 2,759 & 3,725 & 1.35 & 18,929 & 25,365 & 1.34 & 55 & 61 & 1.11 & 636 & 611 & .96 & 1,635 & 1,439 & .88 & 21,705 & 23,876 & 1.10 \\
\hline 1995 & 3,165 & 3,450 & 1.09 & 14,046 & 16,855 & 1.20 & 160 & 170 & 1.06 & 567 & 425 & .75 & 1,615 & 888 & .55 & 23,251 & 18,833 & .81 \\
\hline 1996 & 3,422 & 3,388 & .99 & 23,068 & 27,451 & 1.19 & 112 & 47 & .42 & 130 & 74 & .57 & 2,479 & 1,735 & .70 & 17,526 & 16,299 & .93 \\
\hline 1997 & 3,537 & 4,068 & 1.15 & 24,188 & 33,379 & 1.38 & 80 & 67 & .84 & 640 & 710 & 1.11 & 2,443 & 1,979 & .81 & 17,606 & 19,367 & 1.10 \\
\hline 1998 & 3,270 & 3,989 & 1.22 & 22,002 & 25,742 & 1.17 & 50 & 83 & 1.66 & 1,923 & 1,731 & .90 & 792 & 475 & .60 & 19,485 & 17,926 & .92 \\
\hline 1999 & 3,075 & 3,137 & 1.02 & 22,493 & 26,767 & 1.19 & 0 & 0 & 0 & 2,133 & 2,112 & .99 & 1,543 & 756 & .49 & 18,349 & 17,065 & .93 \\
\hline 2000 & 2,484 & 3,627 & 1.46 & 22,851 & 34,048 & 1.49 & 134 & 66 & .49 & 1,793 & 2,492 & 1.39 & 1,642 & 1,067 & .65 & 20,315 & 23,972 & 1.18 \\
\hline 2001 & 2,543 & 3,331 & 1.31 & 17,744 & 22,003 & 1.24 & 890 & 1,077 & 1.21 & 2,926 & 2,955 & 1.01 & 1,841 & 939 & .51 & 20,949 & 20,530 & .98 \\
\hline 2002 & 2,496 & 3,844 & 1.54 & 18,256 & 26,836 & 1.47 & 632 & 455 & .72 & 2,769 & 4,237 & 1.53 & 880 & 572 & .65 & 21,543 & 29,298 & 1.36 \\
\hline 2003 & 2,391 & 3,300 & 1.38 & 13,936 & 19,928 & 1.43 & 1,314 & 1,472 & 1.12 & 2,415 & 3,429 & 1.42 & 3,811 & 2,973 & .78 & 22,719 & 26,581 & 1.17 \\
\hline Average & 2,958 & 3,680 & 1.26 & 19,306 & 24,669 & 1.28 & 651 & 675 & .89 & 1,408 & 1,611 & 1.03 & 1,860 & 1,289 & .67 & 19,821 & 20,607 & 1.03 \\
\hline
\end{tabular}


Table 2. Irrigation water use, acres irrigated, and irrigation application rate by crop type for counties in the Groundwater Management Districts within the Kansas High Plains, 1991-2003-Continued

[Data from Kansas Water Office and Kansas Department of Agriculture (1993-2005). acre-ft, acre-foot; acre-ft/acre, acre-foot per acre]

\begin{tabular}{|c|c|c|c|c|c|c|c|c|c|c|c|c|c|c|c|c|c|c|}
\hline \multirow[b]{2}{*}{ Year } & \multicolumn{3}{|c|}{ Alfalfa } & \multicolumn{3}{|c|}{ Corn } & \multicolumn{3}{|c|}{ Grain sorghum } & \multicolumn{3}{|c|}{ Soybeans } & \multicolumn{3}{|c|}{ Wheat } & \multicolumn{3}{|c|}{ Other/Multiple use } \\
\hline & $\begin{array}{c}\text { Total } \\
\text { acres } \\
\text { irrigated }\end{array}$ & $\begin{array}{c}\text { Irriga- } \\
\text { tion } \\
\text { water } \\
\text { used } \\
\text { (acre-ft) }\end{array}$ & $\begin{array}{l}\text { Rate of } \\
\text { irri- } \\
\text { gation } \\
\text { (acre- } \\
\text { ft/acre) }\end{array}$ & $\begin{array}{c}\text { Total } \\
\text { acres } \\
\text { irrigated }\end{array}$ & $\begin{array}{c}\text { Irriga- } \\
\text { tion } \\
\text { water } \\
\text { used } \\
\text { (acre-ft) }\end{array}$ & $\begin{array}{c}\text { Rate of } \\
\text { irri- } \\
\text { gation } \\
\text { (acre- } \\
\text { ft/acre) }\end{array}$ & $\begin{array}{c}\text { Total } \\
\text { acres } \\
\text { irrigated }\end{array}$ & $\begin{array}{c}\text { Irriga- } \\
\text { tion } \\
\text { water } \\
\text { used } \\
\text { (acre-ft) }\end{array}$ & $\begin{array}{c}\text { Rate of } \\
\text { irri- } \\
\text { gation } \\
\text { (acre- } \\
\text { ft/acre) }\end{array}$ & $\begin{array}{c}\text { Total } \\
\text { acres } \\
\text { irrigated }\end{array}$ & $\begin{array}{c}\text { Irriga- } \\
\text { tion } \\
\text { water } \\
\text { used } \\
\text { (acre-ft) }\end{array}$ & $\begin{array}{c}\text { Rate of } \\
\text { irri- } \\
\text { gation } \\
\text { (acre- } \\
\text { ft/acre) }\end{array}$ & $\begin{array}{c}\text { Total } \\
\text { acres } \\
\text { irrigated }\end{array}$ & $\begin{array}{l}\text { Irriga- } \\
\text { tion } \\
\text { water } \\
\text { used } \\
\text { (acre-ft) }\end{array}$ & $\begin{array}{l}\text { Rate of } \\
\text { irri- } \\
\text { gation } \\
\text { (acre- } \\
\text { ft/acre) }\end{array}$ & $\begin{array}{c}\text { Total } \\
\text { acres } \\
\text { irrigated }\end{array}$ & $\begin{array}{c}\text { Irriga- } \\
\text { tion } \\
\text { water } \\
\text { used } \\
\text { (acre-ft) }\end{array}$ & $\begin{array}{l}\text { Rate of } \\
\text { irri- } \\
\text { gation } \\
\text { (acre- } \\
\text { ft/acre) }\end{array}$ \\
\hline \multicolumn{19}{|c|}{ Edwards County } \\
\hline 1991 & 15,697 & 23,389 & 1.49 & 39,844 & 59,368 & 1.49 & 2,359 & 2,878 & 1.22 & 9,371 & 12,932 & 1.38 & 4,315 & 3,323 & 0.77 & 23,247 & 32,778 & 1.41 \\
\hline 1992 & 13,843 & 13,012 & .94 & 48,574 & 42,259 & .87 & 2,774 & 1,664 & .60 & 5,925 & 4,740 & .80 & 4,021 & 2,332 & .58 & 19,664 & 15,535 & .79 \\
\hline 1993 & 12,876 & 9,271 & .72 & 46,277 & 42,112 & .91 & 1,108 & 565 & .51 & 6,036 & 4,829 & .80 & 2,183 & 677 & .31 & 24,223 & 17,925 & .74 \\
\hline 1994 & 12,767 & 17,618 & 1.38 & 47,295 & 66,686 & 1.41 & 987 & 957 & .97 & 8,387 & 10,316 & 1.23 & 1,427 & 1,241 & .87 & 22,156 & 28,138 & 1.27 \\
\hline 1995 & 12,844 & 14,000 & 1.09 & 47,498 & 57,473 & 1.21 & 716 & 630 & .88 & 8,052 & 9,340 & 1.16 & 2,154 & 991 & .46 & 21,571 & 22,218 & 1.03 \\
\hline 1996 & 10,887 & 8,274 & .76 & 55,345 & 45,936 & .83 & 970 & 349 & .36 & 8,684 & 5,905 & .68 & 1,369 & 561 & .41 & 17,636 & 13,932 & .79 \\
\hline 1997 & 13,425 & 11,948 & .89 & 48,336 & 40,602 & .84 & 1,304 & 600 & .46 & 16,457 & 12,672 & .77 & 1,314 & 394 & .30 & 15,920 & 11,462 & .72 \\
\hline 1998 & 10,903 & 14,392 & 1.32 & 48,038 & 62,930 & 1.31 & 1,150 & 1,748 & 1.52 & 18,438 & 23,969 & 1.30 & 776 & 194 & .25 & 18,666 & 23,146 & 1.24 \\
\hline 1999 & 12,005 & 12,485 & 1.04 & 46,959 & 52,124 & 1.11 & 1,467 & 1,467 & 1.00 & 16,982 & 20,378 & 1.20 & 1,810 & 778 & .43 & 20,842 & 21,676 & 1.04 \\
\hline 2000 & 8,898 & 11,478 & 1.29 & 54,547 & 69,275 & 1.27 & 794 & 691 & .87 & 18,118 & 24,097 & 1.33 & 1,490 & 894 & .60 & 16,184 & 19,583 & 1.21 \\
\hline 2001 & 9,773 & 12,509 & 1.28 & 50,421 & 67,060 & 1.33 & 1,115 & 702 & .63 & 13,719 & 19,207 & 1.40 & 2,204 & 1,829 & .83 & 22,554 & 26,163 & 1.16 \\
\hline 2002 & 12,702 & 17,275 & 1.36 & 51,654 & 70,766 & 1.37 & 1,141 & 993 & .87 & 12,272 & 17,672 & 1.44 & 2,598 & 1,819 & .70 & 20,927 & 25,740 & 1.23 \\
\hline 2003 & 12,289 & 14,624 & 1.19 & 45,524 & 62,823 & 1.38 & 1,828 & 1,408 & .77 & 12,932 & 17,717 & 1.37 & 3,284 & 1,773 & .54 & 24,670 & 29,357 & 1.19 \\
\hline Average & 12,224 & 13,867 & 1.13 & 48,486 & 56,878 & 1.18 & 1,363 & 1,127 & .82 & 11,952 & 14,136 & 1.14 & 2,227 & 1,293 & .54 & 20,635 & 22,127 & 1.06 \\
\hline \multicolumn{19}{|c|}{ Finney County } \\
\hline 1991 & 28,061 & 51,071 & 1.82 & 39,728 & 75,880 & 1.91 & 6,979 & 9,910 & 1.42 & 3,598 & 5,541 & 1.54 & 22,420 & 25,335 & 1.13 & 152,571 & 241,062 & 1.58 \\
\hline 1992 & 27,788 & 41,126 & 1.48 & 42,285 & 65,119 & 1.54 & 5,052 & 4,395 & .87 & 6,028 & 5,847 & .97 & 24,885 & 27,871 & 1.12 & 144,156 & 145,598 & 1.01 \\
\hline 1993 & 29,270 & 42,149 & 1.44 & 46,346 & 69,982 & 1.51 & 2,516 & 2,113 & .84 & 2,855 & 2,912 & 1.02 & 21,049 & 19,786 & .94 & 139,375 & 129,619 & .93 \\
\hline 1994 & 36,202 & 65,888 & 1.82 & 42,596 & 70,709 & 1.66 & 4,577 & 6,591 & 1.44 & 2,458 & 4,154 & 1.69 & 16,834 & 22,726 & 1.35 & 140,418 & 178,331 & 1.27 \\
\hline 1995 & 37,740 & 59,629 & 1.58 & 49,436 & 76,626 & 1.55 & 3,220 & 3,478 & 1.08 & 1,857 & 2,563 & 1.38 & 13,060 & 10,840 & .83 & 137,985 & 155,923 & 1.13 \\
\hline 1996 & 33,362 & 49,709 & 1.49 & 60,964 & 89,007 & 1.46 & 1,994 & 1,236 & .62 & 759 & 592 & .78 & 9,966 & 7,973 & .80 & 133,238 & 147,894 & 1.11 \\
\hline 1997 & 38,902 & 62,243 & 1.60 & 61,271 & 85,167 & 1.39 & 2,438 & 2,072 & .85 & 2,093 & 1,967 & .94 & 10,614 & 9,765 & .92 & 127,812 & 131,646 & 1.03 \\
\hline 1998 & 42,314 & 70,664 & 1.67 & 60,268 & 87,389 & 1.45 & 1,137 & 796 & .70 & 1,945 & 2,820 & 1.45 & 9,504 & 7,603 & .80 & 125,788 & 125,788 & 10 \\
\hline 1999 & 42,257 & 59,582 & 1.41 & 56,376 & 77,799 & 1.38 & 2,414 & 2,486 & 1.03 & 2,325 & 2,604 & 1.12 & 12,167 & 7,300 & .60 & 126,095 & 113,486 & .90 \\
\hline 2000 & 37,547 & 71,339 & 1.90 & 55,670 & 93,526 & 1.68 & 1,174 & 845 & .72 & 4,417 & 7,067 & 1.60 & 8,022 & 6,658 & .83 & 129,450 & 142,395 & 1.10 \\
\hline 2001 & 50,557 & 81,902 & 1.62 & 44,233 & 61,926 & 1.40 & 1,572 & 1,289 & .82 & 6,989 & 11,392 & 1.63 & 13,890 & 11,251 & .81 & 124,472 & 117,004 & .94 \\
\hline 2002 & 54,458 & 98,024 & 1.80 & 39,774 & 70,002 & 1.76 & 2,817 & 2,732 & .97 & 6,997 & 12,595 & 1.80 & 9,842 & 9,251 & .94 & 130,408 & 177,355 & 1.36 \\
\hline 2003 & 50,167 & 82,776 & 1.65 & 35,070 & 53,657 & 1.53 & 4,020 & 4,543 & 1.13 & 8,304 & 13,536 & 1.63 & 9,018 & 8,477 & .94 & 128,486 & 159,323 & 1.24 \\
\hline Average & 39,125 & 64,316 & 1.64 & 48,771 & 75,138 & 1.56 & 3,070 & 3,268 & .96 & 3,894 & 5,661 & 1.35 & 13,944 & 13,449 & .92 & 133,866 & 151,186 & 1.12 \\
\hline
\end{tabular}


Table 2. Irrigation water use, acres irrigated, and irrigation application rate by crop type for counties in the Groundwater Management Districts within the Kansas High Plains, 1991-2003-Continued

[Data from Kansas Water Office and Kansas Department of Agriculture (1993-2005). acre-ft, acre-foot; acre-ft/acre, acre-foot per acre]

\begin{tabular}{|c|c|c|c|c|c|c|c|c|c|c|c|c|c|c|c|c|c|c|}
\hline \multirow[b]{2}{*}{ Year } & \multicolumn{3}{|c|}{ Alfalfa } & \multicolumn{3}{|c|}{ Corn } & \multicolumn{3}{|c|}{ Grain sorghum } & \multicolumn{3}{|c|}{ Soybeans } & \multicolumn{3}{|c|}{ Wheat } & \multicolumn{3}{|c|}{ Other/Multiple use } \\
\hline & $\begin{array}{c}\text { Total } \\
\text { acres } \\
\text { irrigated }\end{array}$ & $\begin{array}{c}\text { Irriga- } \\
\text { tion } \\
\text { water } \\
\text { used } \\
\text { (acre-ft) }\end{array}$ & $\begin{array}{c}\text { Rate of } \\
\text { irri- } \\
\text { gation } \\
\text { (acre- } \\
\text { ft/acre) }\end{array}$ & $\begin{array}{c}\text { Total } \\
\text { acres } \\
\text { irrigated }\end{array}$ & $\begin{array}{l}\text { Irriga- } \\
\text { tion } \\
\text { water } \\
\text { used } \\
\text { (acre-ft) }\end{array}$ & $\begin{array}{l}\text { Rate of } \\
\text { irri- } \\
\text { gation } \\
\text { (acre- } \\
\text { ft/acre) }\end{array}$ & $\begin{array}{c}\text { Total } \\
\text { acres } \\
\text { irrigated }\end{array}$ & $\begin{array}{l}\text { Irriga- } \\
\text { tion } \\
\text { water } \\
\text { used } \\
\text { (acre-ft) }\end{array}$ & $\begin{array}{l}\text { Rate of } \\
\text { irri- } \\
\text { gation } \\
\text { (acre- } \\
\text { ft/acre) }\end{array}$ & $\begin{array}{c}\text { Total } \\
\text { acres } \\
\text { irrigated }\end{array}$ & $\begin{array}{l}\text { Irriga- } \\
\text { tion } \\
\text { water } \\
\text { used } \\
\text { (acre-ft) }\end{array}$ & $\begin{array}{l}\text { Rate of } \\
\text { irri- } \\
\text { gation } \\
\text { (acre- } \\
\text { ft/acre) }\end{array}$ & $\begin{array}{c}\text { Total } \\
\text { acres } \\
\text { irrigated }\end{array}$ & $\begin{array}{l}\text { Irriga- } \\
\text { tion } \\
\text { water } \\
\text { used } \\
\text { (acre-ft) }\end{array}$ & $\begin{array}{l}\text { Rate of } \\
\text { irri- } \\
\text { gation } \\
\text { (acre- } \\
\text { ft/acre) }\end{array}$ & $\begin{array}{c}\text { Total } \\
\text { acres } \\
\text { irrigated }\end{array}$ & $\begin{array}{l}\text { Irriga- } \\
\text { tion } \\
\text { water } \\
\text { used } \\
\text { (acre-ft) }\end{array}$ & $\begin{array}{c}\text { Rate of } \\
\text { irri- } \\
\text { gation } \\
\text { (acre- } \\
\text { ft/acre) }\end{array}$ \\
\hline \multicolumn{19}{|c|}{ Ford County } \\
\hline 1991 & 10,562 & 19,645 & 1.86 & 19,634 & 32,789 & 1.67 & 10,468 & 13,504 & 1.29 & 2,034 & 2,990 & 1.47 & 6,261 & 5,572 & 0.89 & 44,341 & 66,955 & 1.51 \\
\hline 1992 & 10,366 & 14,098 & 1.36 & 20,819 & 23,942 & 1.15 & 11,533 & 9,572 & .83 & 1,963 & 1,727 & .88 & 5,165 & 3,512 & .68 & 39,981 & 39,581 & .99 \\
\hline 1993 & 8,577 & 11,322 & 1.32 & 27,166 & 31,513 & 1.16 & 8,517 & 6,217 & .73 & 1,219 & 939 & .77 & 4,001 & 1,760 & .44 & 39,543 & 36,775 & .93 \\
\hline 1994 & 8,133 & 13,419 & 1.65 & 31,065 & 43,180 & 1.39 & 5,461 & 4,423 & .81 & 1,793 & 1,667 & .93 & 5,061 & 2,834 & .56 & 40,185 & 45,811 & 1.14 \\
\hline 1995 & 7,529 & 11,068 & 1.47 & 33,583 & 41,979 & 1.25 & 4,262 & 3,921 & .92 & 1,216 & 1,313 & 1.08 & 3,570 & 2,142 & .60 & 39,494 & 39,889 & 1.01 \\
\hline 1996 & 8,201 & 10,497 & 1.28 & 36,360 & 39,632 & 1.09 & 3,524 & 2,150 & .61 & 1,819 & 982 & .54 & 2,582 & 1,291 & .50 & 36,861 & 27,646 & .75 \\
\hline 1997 & 6,996 & 10,284 & 1.47 & 41,947 & 38,172 & .91 & 3,957 & 2,572 & .65 & 962 & 789 & .82 & 1,969 & 847 & .43 & 31,107 & 21,775 & .70 \\
\hline 1998 & 7,240 & 11,584 & 1.60 & 39,793 & 50,935 & 1.28 & 1,616 & 1,293 & .80 & 2,769 & 3,517 & 1.27 & 1,631 & 669 & .41 & 33,659 & 31,976 & .95 \\
\hline 1999 & 6,439 & 9,272 & 1.44 & 37,258 & 40,984 & 1.10 & 1,537 & 999 & .65 & 3,534 & 3,887 & 1.10 & 2,169 & 976 & .45 & 36,673 & 33,372 & .91 \\
\hline 2000 & 5,766 & 9,283 & 1.61 & 39,375 & 51,975 & 1.32 & 1,158 & 753 & .65 & 4,369 & 5,854 & 1.34 & 2,285 & 1,417 & .62 & 37,403 & 35,533 & .95 \\
\hline 2001 & 6,345 & 10,723 & 1.69 & 34,810 & 44,905 & 1.29 & 1,607 & 1,286 & .80 & 5,957 & 7,923 & 1.33 & 2,451 & 1,765 & .72 & 40,306 & 41,112 & 1.02 \\
\hline 2002 & 6,349 & 9,587 & 1.51 & 31,391 & 45,831 & 1.46 & 4,187 & 3,601 & .86 & 5,687 & 7,848 & 1.38 & 2,008 & 1,667 & .83 & 43,774 & 47,276 & 1.08 \\
\hline 2003 & 6,552 & 9,042 & 1.38 & 28,767 & 38,835 & 1.35 & 5,143 & 4,474 & .87 & 4,421 & 5,615 & 1.27 & 4,687 & 2,859 & .61 & 40,459 & 37,627 & .93 \\
\hline Average & 7,620 & 11,525 & 1.51 & 32,459 & 40,359 & 1.26 & 4,844 & 4,213 & .81 & 2,903 & 3,466 & 1.09 & 3,372 & 2,101 & .60 & 38,753 & 38,871 & .99 \\
\hline \multicolumn{19}{|c|}{ Graham County } \\
\hline 1991 & 1,133 & 1,620 & 1.43 & 2,241 & 4,191 & 1.87 & 1,320 & 1,333 & 1.01 & 121 & 172 & 1.42 & 1,255 & 540 & .43 & 6,269 & 7,774 & 1.24 \\
\hline 1992 & 1,162 & 616 & .53 & 1,487 & 1,130 & .76 & 1,396 & 530 & .38 & 430 & 576 & 1.34 & 900 & 306 & .34 & 5,789 & 3,473 & .60 \\
\hline 1993 & 1,712 & 308 & .18 & 3,201 & 1,921 & .60 & 342 & 41 & .12 & 125 & 23 & .18 & 397 & 52 & .13 & 3,111 & 778 & .25 \\
\hline 1994 & 1,656 & 1,275 & .77 & 5,598 & 6,718 & 1.20 & 645 & 258 & .40 & 50 & 40 & .79 & 434 & 169 & .39 & 2,929 & 1,816 & .62 \\
\hline 1995 & 1,451 & 1,146 & .79 & 5,616 & 6,739 & 1.20 & 315 & 192 & .61 & 0 & 0 & 0 & 822 & 288 & .35 & 3,170 & 2,441 & .77 \\
\hline 1996 & 1,310 & 812 & .62 & 7,132 & 6,918 & .97 & 258 & 124 & .48 & 0 & 0 & 0 & 898 & 314 & .35 & 2,566 & 1,540 & .60 \\
\hline 1997 & 1,598 & 1,183 & .74 & 7,721 & 8,416 & 1.09 & 274 & 55 & .20 & 45 & 34 & .76 & 547 & 164 & .30 & 2,419 & 1,597 & .66 \\
\hline 1998 & 1,662 & 1,263 & .76 & 8,934 & 8,666 & .97 & 498 & 239 & .48 & 127 & 98 & .77 & 768 & 200 & .26 & 1,775 & 1,065 & .60 \\
\hline 1999 & 1,559 & 1,060 & .68 & 8,104 & 6,078 & .75 & 303 & 33 & .11 & 555 & 427 & .77 & 819 & 278 & .34 & 2,498 & 1,749 & .70 \\
\hline 2000 & 1,625 & 1,381 & .85 & 8,215 & 9,283 & 1.13 & 245 & 164 & .67 & 728 & 786 & 1.08 & 614 & 252 & .41 & 3,163 & 2,815 & .89 \\
\hline 2001 & 2,174 & 1,891 & .87 & 5,751 & 5,693 & .99 & 495 & 203 & .41 & 600 & 522 & .87 & 649 & 208 & .32 & 4,429 & 3,455 & .78 \\
\hline 2002 & 1,891 & 2,647 & 1.40 & 5,891 & 7,540 & 1.28 & 500 & 510 & 1.02 & 728 & 655 & .90 & 822 & 238 & .29 & 4,942 & 5,535 & 1.12 \\
\hline 2003 & 2,204 & 2,182 & .99 & 4,186 & 4,772 & 1.14 & 681 & 674 & .99 & 733 & 931 & 1.27 & 1,402 & 771 & .55 & 5,553 & 6,053 & 1.09 \\
\hline Average & 1,626 & 1,337 & .82 & 5,698 & 6,005 & 1.07 & 559 & 335 & .53 & 326 & 328 & .78 & 794 & 291 & .34 & 3,739 & 3,084 & .76 \\
\hline
\end{tabular}


Table 2. Irrigation water use, acres irrigated, and irrigation application rate by crop type for counties in the Groundwater Management Districts within the Kansas High Plains, 1991-2003-Continued

[Data from Kansas Water Office and Kansas Department of Agriculture (1993-2005). acre-ft, acre-foot; acre-ft/acre, acre-foot per acre]

\begin{tabular}{|c|c|c|c|c|c|c|c|c|c|c|c|c|c|c|c|c|c|c|}
\hline \multirow[b]{2}{*}{ Year } & \multicolumn{3}{|c|}{ Alfalfa } & \multicolumn{3}{|c|}{ Corn } & \multicolumn{3}{|c|}{ Grain sorghum } & \multicolumn{3}{|c|}{ Soybeans } & \multicolumn{3}{|c|}{ Wheat } & \multicolumn{3}{|c|}{ Other/Multiple use } \\
\hline & $\begin{array}{c}\text { Total } \\
\text { acres } \\
\text { irrigated }\end{array}$ & $\begin{array}{c}\text { Irriga- } \\
\text { tion } \\
\text { water } \\
\text { used } \\
\text { (acre-ft) }\end{array}$ & $\begin{array}{l}\text { Rate of } \\
\text { irri- } \\
\text { gation } \\
\text { (acre- } \\
\text { ft/acre) }\end{array}$ & $\begin{array}{c}\text { Total } \\
\text { acres } \\
\text { irrigated }\end{array}$ & $\begin{array}{c}\text { Irriga- } \\
\text { tion } \\
\text { water } \\
\text { used } \\
\text { (acre-ft) }\end{array}$ & $\begin{array}{c}\text { Rate of } \\
\text { irri- } \\
\text { gation } \\
\text { (acre- } \\
\text { ft/acre) }\end{array}$ & $\begin{array}{c}\text { Total } \\
\text { acres } \\
\text { irrigated }\end{array}$ & $\begin{array}{l}\text { Irriga- } \\
\text { tion } \\
\text { water } \\
\text { used } \\
\text { (acre-ft) }\end{array}$ & $\begin{array}{l}\text { Rate of } \\
\text { irri- } \\
\text { gation } \\
\text { (acre- } \\
\text { ft/acre) }\end{array}$ & $\begin{array}{c}\text { Total } \\
\text { acres } \\
\text { irrigated }\end{array}$ & $\begin{array}{c}\text { Irriga- } \\
\text { tion } \\
\text { water } \\
\text { used } \\
\text { (acre-ft) }\end{array}$ & $\begin{array}{l}\text { Rate of } \\
\text { irri- } \\
\text { gation } \\
\text { (acre- } \\
\text { ft/acre) }\end{array}$ & $\begin{array}{c}\text { Total } \\
\text { acres } \\
\text { irrigated }\end{array}$ & $\begin{array}{c}\text { Irriga- } \\
\text { tion } \\
\text { water } \\
\text { used } \\
\text { (acre-ft) }\end{array}$ & $\begin{array}{l}\text { Rate of } \\
\text { irri- } \\
\text { gation } \\
\text { (acre- } \\
\text { ft/acre) }\end{array}$ & $\begin{array}{c}\text { Total } \\
\text { acres } \\
\text { irrigated }\end{array}$ & $\begin{array}{l}\text { Irriga- } \\
\text { tion } \\
\text { water } \\
\text { used } \\
\text { (acre-ft) }\end{array}$ & $\begin{array}{c}\text { Rate of } \\
\text { irri- } \\
\text { gation } \\
\text { (acre- } \\
\text { ft/acre) }\end{array}$ \\
\hline \multicolumn{19}{|c|}{ Grant County } \\
\hline 1991 & 8,614 & 18,089 & 2.10 & 7,769 & 15,538 & 20 & 1,586 & 2,236 & 1.41 & 4 & 5 & 1.13 & 3,862 & 4,364 & 1.13 & 120,203 & 195,931 & 1.63 \\
\hline 1992 & 7,702 & 13,633 & 1.77 & 10,440 & 18,061 & 1.73 & 1,934 & 1,663 & .86 & 0 & 0 & 0 & 3,584 & 4,767 & 1.33 & 120,226 & 174,328 & 1.45 \\
\hline 1993 & 6,654 & 11,112 & 1.67 & 13,878 & 22,344 & 1.61 & 610 & 323 & .53 & 162 & 183 & 1.13 & 2,959 & 2,042 & .69 & 114,489 & 122,503 & 1.07 \\
\hline 1994 & 5,127 & 9,741 & 1.90 & 13,000 & 24,440 & 1.88 & 362 & 442 & 1.22 & 0 & 0 & 0 & 2,938 & 2,204 & .75 & 117,669 & 160,030 & 1.36 \\
\hline 1995 & 5,375 & 10,428 & 1.94 & 12,513 & 22,774 & 1.82 & 396 & 693 & 1.75 & 0 & 0 & 0 & 1,831 & 1,996 & 1.09 & 116,090 & 150,917 & 1.30 \\
\hline 1996 & 4,716 & 7,876 & 1.67 & 15,002 & 23,853 & 1.59 & 586 & 498 & .85 & 0 & 0 & 0 & 1,904 & 1,809 & .95 & 112,020 & 138,905 & 1.24 \\
\hline 1997 & 5,865 & 9,149 & 1.56 & 16,471 & 21,906 & 1.33 & 470 & 334 & .71 & 875 & 1,050 & 1.20 & 2,818 & 2,085 & .74 & 111,204 & 110,092 & .99 \\
\hline 1998 & 5,975 & 10,456 & 1.75 & 16,257 & 23,898 & 1.47 & 290 & 55 & .19 & 410 & 578 & 1.41 & 3,282 & 2,658 & .81 & 108,769 & 105,506 & .97 \\
\hline 1999 & 5,816 & 8,724 & 1.50 & 20,423 & 31,043 & 1.52 & 874 & 507 & .58 & 130 & 221 & 1.70 & 2,533 & 1,950 & .77 & 100,790 & 101,798 & 1.01 \\
\hline 2000 & 5,977 & 11,834 & 1.98 & 23,836 & 39,329 & 1.65 & 1,321 & 1,110 & .84 & 245 & 453 & 1.85 & 2,601 & 1,743 & .67 & 92,949 & 113,398 & 1.22 \\
\hline 2001 & 5,441 & 8,216 & 1.51 & 19,417 & 27,572 & 1.42 & 1,187 & 985 & .83 & 125 & 83 & .66 & 6,834 & 3,622 & .53 & 86,980 & 80,891 & .93 \\
\hline 2002 & 7,115 & 13,590 & 1.91 & 16,820 & 31,790 & 1.89 & 1,535 & 1,489 & .97 & 0 & 0 & 0 & 3,260 & 2,119 & .65 & 92,131 & 117,928 & 1.28 \\
\hline 2003 & 7,882 & 13,557 & 1.72 & 16,022 & 26,597 & 1.66 & 2,690 & 1,883 & .70 & 160 & 250 & 1.56 & 2,430 & 1,507 & .62 & 88,048 & 100,375 & 1.14 \\
\hline Average & 6,328 & 11,262 & 1.77 & 15,527 & 25,319 & 1.66 & 1,065 & 940 & .88 & 162 & 217 & .82 & 3,141 & 2,528 & .83 & 106,274 & 128,662 & 1.20 \\
\hline \multicolumn{19}{|c|}{ Gray County } \\
\hline 1991 & 24,592 & 51,397 & 2.09 & 34,239 & 66,766 & 1.95 & 11,566 & 16,077 & 1.39 & 2,576 & 3,916 & 1.52 & 19,224 & 19,801 & 1.03 & 105,455 & 160,292 & 1.52 \\
\hline 1992 & 24,540 & 39,509 & 1.61 & 38,420 & 54,556 & 1.42 & 8,438 & 7,932 & .94 & 1,444 & 1,473 & 1.02 & 16,723 & 15,552 & .93 & 103,250 & 115,640 & 1.12 \\
\hline 1993 & 24,287 & 35,702 & 1.47 & 47,071 & 66,841 & 1.42 & 7,559 & 5,820 & .77 & 1,843 & 1,972 & 1.07 & 15,248 & 10,369 & .68 & 91,741 & 89,906 & .98 \\
\hline 1994 & 22,098 & 38,672 & 1.75 & 49,379 & 81,969 & 1.66 & 5,196 & 5,092 & .98 & 1,619 & 1,943 & 1.20 & 12,992 & 8,055 & .62 & 98,573 & 120,259 & 1.22 \\
\hline 1995 & 24,293 & 38,140 & 1.57 & 49,422 & 81,052 & 1.64 & 5,238 & 4,295 & .82 & 1,406 & 1,982 & 1.41 & 11,792 & 7,547 & .64 & 99,250 & 116,123 & 1.17 \\
\hline 1996 & 24,329 & 31,871 & 1.31 & 60,893 & 82,814 & 1.36 & 4,484 & 3,139 & .70 & 1,057 & 1,194 & 1.13 & 9,147 & 6,586 & .72 & 88,358 & 91,892 & 1.04 \\
\hline 1997 & 26,909 & 43,323 & 1.61 & 68,948 & 86,874 & 1.26 & 3,123 & 2,061 & .66 & 1,649 & 1,847 & 1.12 & 9,363 & 7,022 & .75 & 83,413 & 82,579 & .99 \\
\hline 1998 & 31,132 & 54,170 & 1.74 & 67,892 & 105,233 & 1.55 & 1,806 & 1,029 & .57 & 3,804 & 5,097 & 1.34 & 7,460 & 5,371 & .72 & 82,775 & 83,603 & 1.01 \\
\hline 1999 & 32,171 & 43,109 & 1.34 & 69,099 & 89,829 & 1.30 & 1,741 & 1,027 & .59 & 6,259 & 5,883 & .94 & 7,714 & 4,011 & .52 & 79,868 & 63,894 & .80 \\
\hline 2000 & 29,646 & 53,066 & 1.79 & 70,610 & 111,564 & 1.58 & 1,649 & 1,468 & .89 & 6,488 & 8,953 & 1.38 & 6,725 & 3,430 & .51 & 81,521 & 83,967 & 1.03 \\
\hline 2001 & 33,119 & 56,633 & 1.71 & 62,654 & 90,848 & 1.45 & 1,758 & 1,565 & .89 & 8,589 & 11,252 & 1.31 & 8,510 & 4,510 & .53 & 78,428 & 77,644 & .99 \\
\hline 2002 & 33,009 & 59,746 & 1.81 & 57,248 & 100,184 & 1.75 & 2,778 & 2,778 & 10 & 6,990 & 10,415 & 1.49 & 8,665 & 5,199 & .60 & 76,036 & 89,722 & 1.18 \\
\hline 2003 & 32,408 & 53,797 & 1.66 & 47,618 & 75,236 & 1.58 & 5,831 & 5,423 & .93 & 6,744 & 8,970 & 1.33 & 11,531 & 7,610 & .66 & 77,294 & 82,705 & 1.07 \\
\hline Average & 27,887 & 46,087 & 1.65 & 55,653 & 84,136 & 1.53 & 4,705 & 4,439 & .86 & 3,882 & 4,992 & 1.25 & 11,161 & 8,082 & .69 & 88,151 & 96,787 & 1.09 \\
\hline
\end{tabular}


Table 2. Irrigation water use, acres irrigated, and irrigation application rate by crop type for counties in the Groundwater Management Districts within the Kansas High Plains, 1991-2003-Continued

[Data from Kansas Water Office and Kansas Department of Agriculture (1993-2005). acre-ft, acre-foot; acre-ft/acre, acre-foot per acre]

\begin{tabular}{|c|c|c|c|c|c|c|c|c|c|c|c|c|c|c|c|c|c|c|}
\hline \multirow[b]{2}{*}{ Year } & \multicolumn{3}{|c|}{ Alfalfa } & \multicolumn{3}{|c|}{ Corn } & \multicolumn{3}{|c|}{ Grain sorghum } & \multicolumn{3}{|c|}{ Soybeans } & \multicolumn{3}{|c|}{ Wheat } & \multicolumn{3}{|c|}{ Other/Multiple use } \\
\hline & $\begin{array}{c}\text { Total } \\
\text { acres } \\
\text { irrigated }\end{array}$ & $\begin{array}{l}\text { Irriga- } \\
\text { tion } \\
\text { water } \\
\text { used } \\
\text { (acre-ft) }\end{array}$ & $\begin{array}{l}\text { Rate of } \\
\text { irri- } \\
\text { gation } \\
\text { (acre- } \\
\text { ft/acre) }\end{array}$ & $\begin{array}{c}\text { Total } \\
\text { acres } \\
\text { irrigated }\end{array}$ & $\begin{array}{l}\text { Irriga- } \\
\text { tion } \\
\text { water } \\
\text { used } \\
\text { (acre-ft) }\end{array}$ & $\begin{array}{l}\text { Rate of } \\
\text { irri- } \\
\text { gation } \\
\text { (acre- } \\
\text { ft/acre) }\end{array}$ & $\begin{array}{c}\text { Total } \\
\text { acres } \\
\text { irrigated }\end{array}$ & $\begin{array}{l}\text { Irriga- } \\
\text { tion } \\
\text { water } \\
\text { used } \\
\text { (acre-ft) }\end{array}$ & $\begin{array}{l}\text { Rate of } \\
\text { irri- } \\
\text { gation } \\
\text { (acre- } \\
\text { ft/acre) }\end{array}$ & $\begin{array}{c}\text { Total } \\
\text { acres } \\
\text { irrigated }\end{array}$ & $\begin{array}{l}\text { Irriga- } \\
\text { tion } \\
\text { water } \\
\text { used } \\
\text { (acre-ft) }\end{array}$ & $\begin{array}{l}\text { Rate of } \\
\text { irri- } \\
\text { gation } \\
\text { (acre- } \\
\text { ft/acre) }\end{array}$ & $\begin{array}{c}\text { Total } \\
\text { acres } \\
\text { irrigated }\end{array}$ & $\begin{array}{l}\text { Irriga- } \\
\text { tion } \\
\text { water } \\
\text { used } \\
\text { (acre-ft) }\end{array}$ & $\begin{array}{l}\text { Rate of } \\
\text { irri- } \\
\text { gation } \\
\text { (acre- } \\
\text { ft/acre) }\end{array}$ & $\begin{array}{c}\text { Total } \\
\text { acres } \\
\text { irrigated }\end{array}$ & $\begin{array}{l}\text { Irriga- } \\
\text { tion } \\
\text { water } \\
\text { used } \\
\text { (acre-ft) }\end{array}$ & $\begin{array}{c}\text { Rate of } \\
\text { irri- } \\
\text { gation } \\
\text { (acre- } \\
\text { ft/acre) }\end{array}$ \\
\hline \multicolumn{19}{|c|}{ Greeley County } \\
\hline 1991 & 7 & 7 & 1.01 & 11,588 & 18,541 & 1.60 & 567 & 1,083 & 1.91 & 0 & 0 & 0 & 497 & 527 & 1.06 & 14,552 & 19,645 & 1.35 \\
\hline 1992 & 102 & 165 & 1.62 & 15,152 & 19,849 & 1.31 & 312 & 222 & .71 & 0 & 0 & 0 & 940 & 620 & .66 & 11,841 & 13,262 & 1.12 \\
\hline 1993 & 0 & 0 & 0 & 14,531 & 16,711 & 1.15 & 430 & 228 & .53 & 0 & 0 & 0 & 122 & 48 & .39 & 11,791 & 11,909 & 1.01 \\
\hline 1994 & 245 & 169 & .69 & 14,774 & 20,684 & 1.40 & 506 & 476 & .94 & 120 & 122 & 1.02 & 555 & 261 & .47 & 10,844 & 13,013 & 1.20 \\
\hline 1995 & 120 & 202 & 1.68 & 14,927 & 15,524 & 1.04 & 80 & 82 & 1.03 & 0 & 0 & 0 & 358 & 372 & 1.04 & 12,544 & 12,042 & .96 \\
\hline 1996 & 245 & 250 & 1.02 & 15,967 & 19,160 & 1.20 & 580 & 238 & .41 & 0 & 0 & 0 & 673 & 478 & .71 & 12,278 & 12,278 & 10 \\
\hline 1997 & 245 & 262 & 1.07 & 16,944 & 17,113 & 1.01 & 119 & 88 & .74 & 120 & 82 & .68 & 808 & 493 & .61 & 10,761 & 10,115 & .94 \\
\hline 1998 & 476 & 457 & .96 & 17,253 & 16,045 & .93 & 60 & 65 & 1.08 & 135 & 173 & 1.28 & 490 & 108 & .22 & 11,953 & 11,116 & .93 \\
\hline 1999 & 477 & 582 & 1.22 & 16,069 & 15,908 & .99 & 314 & 195 & .62 & 125 & 128 & 1.02 & 550 & 17 & .03 & 12,205 & 9,398 & .77 \\
\hline 2000 & 519 & 862 & 1.66 & 14,210 & 16,484 & 1.16 & 353 & 441 & 1.25 & 115 & 16 & .14 & 728 & 546 & .75 & 13,526 & 13,526 & 10 \\
\hline 2001 & 350 & 648 & 1.85 & 9,444 & 11,616 & 1.23 & 979 & 920 & .94 & 0 & 0 & 0 & 925 & 453 & .49 & 16,766 & 14,419 & .86 \\
\hline 2002 & 599 & 1,174 & 1.96 & 9,021 & 12,900 & 1.43 & 466 & 489 & 1.05 & 0 & 0 & 0 & 1,315 & 1,328 & 1.01 & 17,860 & 17,860 & 10 \\
\hline 2003 & 377 & 622 & 1.65 & 4,581 & 6,184 & 1.35 & 490 & 358 & .73 & 125 & 15 & .12 & 1,356 & 746 & .55 & 21,509 & 20,649 & .96 \\
\hline Average & 289 & 415 & 1.26 & 13,420 & 15,902 & 1.22 & 404 & 376 & .92 & 57 & 41 & .33 & 717 & 461 & .61 & 13,725 & 13,787 & 1.01 \\
\hline \multicolumn{19}{|c|}{ Harvey County } \\
\hline 1991 & 124 & 77 & .62 & 5,878 & 8,053 & 1.37 & 1,330 & 1,011 & .76 & 2,941 & 3,176 & 1.08 & 392 & 318 & .81 & 16,672 & 20,006 & 1.20 \\
\hline 1992 & 188 & 107 & .57 & 4,847 & 3,296 & .68 & 1,907 & 1,201 & .63 & 2,796 & 2,125 & .76 & 131 & 97 & .74 & 16,342 & 11,766 & .72 \\
\hline 1993 & 163 & 64 & .39 & 4,506 & 3,289 & .73 & 798 & 279 & .35 & 2,698 & 2,050 & .76 & 0 & 0 & 0 & 17,204 & 11,183 & .65 \\
\hline 1994 & 411 & 469 & 1.14 & 4,569 & 5,391 & 1.18 & 1,006 & 865 & .86 & 4,372 & 4,591 & 1.05 & 22 & 2 & .08 & 16,498 & 17,818 & 1.08 \\
\hline 1995 & 103 & 131 & 1.27 & 5,048 & 5,199 & 1.03 & 2,252 & 1,689 & .75 & 3,575 & 3,146 & .88 & 300 & 78 & .26 & 16,423 & 14,452 & .88 \\
\hline 1996 & 46 & 15 & .32 & 6,324 & 6,830 & 1.08 & 1,376 & 963 & .70 & 3,416 & 2,938 & .86 & 218 & 76 & .35 & 17,480 & 14,159 & .81 \\
\hline 1997 & 130 & 243 & 1.87 & 7,201 & 5,689 & .79 & 734 & 477 & .65 & 3,892 & 2,685 & .69 & 272 & 71 & .26 & 17,161 & 12,184 & .71 \\
\hline 1998 & 410 & 533 & 1.30 & 6,882 & 6,676 & .97 & 629 & 346 & .55 & 4,893 & 4,306 & .88 & 27 & 8 & .30 & 17,285 & 15,557 & .90 \\
\hline 1999 & 30 & 8 & .25 & 7,547 & 5,736 & .76 & 612 & 306 & .50 & 6,354 & 4,893 & .77 & 67 & 7 & .11 & 15,390 & 12,312 & .80 \\
\hline 2000 & 30 & 11 & .37 & 6,461 & 5,944 & .92 & 532 & 399 & .75 & 7,958 & 8,674 & 1.09 & 60 & 3 & .05 & 16,391 & 15,571 & .95 \\
\hline 2001 & 0 & 0 & 0 & 7,053 & 8,252 & 1.17 & 869 & 626 & .72 & 6,872 & 8,040 & 1.17 & 200 & 204 & 1.02 & 16,187 & 18,615 & 1.15 \\
\hline 2002 & 20 & 9 & .44 & 8,154 & 9,051 & 1.11 & 591 & 349 & .59 & 6,346 & 6,663 & 1.05 & 0 & 0 & 0 & 16,733 & 17,235 & 1.03 \\
\hline 2003 & 89 & 72 & .81 & 10,606 & 13,045 & 1.23 & 1,688 & 1,148 & .68 & 4,954 & 5,449 & 1.10 & 60 & 1 & .01 & 15,565 & 17,744 & 1.14 \\
\hline Average & 134 & 134 & .72 & 6,544 & 6,650 & 10 & 1,102 & 743 & .65 & 4,697 & 4,518 & .93 & 135 & 66 & .31 & 16,564 & 15,277 & .92 \\
\hline
\end{tabular}


Table 2. Irrigation water use, acres irrigated, and irrigation application rate by crop type for counties in the Groundwater Management Districts within the Kansas High Plains, 1991-2003-Continued

[Data from Kansas Water Office and Kansas Department of Agriculture (1993-2005). acre-ft, acre-foot; acre-ft/acre, acre-foot per acre]

\begin{tabular}{|c|c|c|c|c|c|c|c|c|c|c|c|c|c|c|c|c|c|c|}
\hline \multirow[b]{2}{*}{ Year } & \multicolumn{3}{|c|}{ Alfalfa } & \multicolumn{3}{|c|}{ Corn } & \multicolumn{3}{|c|}{ Grain sorghum } & \multicolumn{3}{|c|}{ Soybeans } & \multicolumn{3}{|c|}{ Wheat } & \multicolumn{3}{|c|}{ Other/Multiple use } \\
\hline & $\begin{array}{c}\text { Total } \\
\text { acres } \\
\text { irrigated }\end{array}$ & $\begin{array}{l}\text { Irriga- } \\
\text { tion } \\
\text { water } \\
\text { used } \\
\text { (acre-ft) }\end{array}$ & $\begin{array}{l}\text { Rate of } \\
\text { irri- } \\
\text { gation } \\
\text { (acre- } \\
\text { ft/acre) }\end{array}$ & $\begin{array}{c}\text { Total } \\
\text { acres } \\
\text { irrigated }\end{array}$ & $\begin{array}{l}\text { Irriga- } \\
\text { tion } \\
\text { water } \\
\text { used } \\
\text { (acre-ft) }\end{array}$ & $\begin{array}{l}\text { Rate of } \\
\text { irri- } \\
\text { gation } \\
\text { (acre- } \\
\text { ft/acre) }\end{array}$ & $\begin{array}{c}\text { Total } \\
\text { acres } \\
\text { irrigated }\end{array}$ & $\begin{array}{l}\text { Irriga- } \\
\text { tion } \\
\text { water } \\
\text { used } \\
\text { (acre-ft) }\end{array}$ & $\begin{array}{l}\text { Rate of } \\
\text { irri- } \\
\text { gation } \\
\text { (acre- } \\
\text { ft/acre) }\end{array}$ & $\begin{array}{c}\text { Total } \\
\text { acres } \\
\text { irrigated }\end{array}$ & $\begin{array}{l}\text { Irriga- } \\
\text { tion } \\
\text { water } \\
\text { used } \\
\text { (acre-ft) }\end{array}$ & $\begin{array}{l}\text { Rate of } \\
\text { irri- } \\
\text { gation } \\
\text { (acre- } \\
\text { ft/acre) }\end{array}$ & $\begin{array}{c}\text { Total } \\
\text { acres } \\
\text { irrigated }\end{array}$ & $\begin{array}{l}\text { Irriga- } \\
\text { tion } \\
\text { water } \\
\text { used } \\
\text { (acre-ft) }\end{array}$ & $\begin{array}{l}\text { Rate of } \\
\text { irri- } \\
\text { gation } \\
\text { (acre- } \\
\mathrm{ft} / \text { acre) }\end{array}$ & $\begin{array}{c}\text { Total } \\
\text { acres } \\
\text { irrigated }\end{array}$ & $\begin{array}{l}\text { Irriga- } \\
\text { tion } \\
\text { water } \\
\text { used } \\
\text { (acre-ft) }\end{array}$ & $\begin{array}{l}\text { Rate of } \\
\text { irri- } \\
\text { gation } \\
\text { (acre- } \\
\text { ft/acre) }\end{array}$ \\
\hline \multicolumn{19}{|c|}{ Haskell County } \\
\hline 1991 & 2,607 & 4,693 & 1.80 & 26,811 & 54,963 & 2.05 & 3,146 & 5,380 & 1.71 & 517 & 822 & 1.59 & 6,260 & 7,387 & 1.18 & 179,325 & 317,405 & 1.77 \\
\hline 1992 & 1,977 & 3,045 & 1.54 & 29,427 & 50,909 & 1.73 & 1,940 & 2,076 & 1.07 & 381 & 431 & 1.13 & 5,285 & 5,549 & 1.05 & 178,235 & 269,135 & 1.51 \\
\hline 1993 & 1,912 & 3,117 & 1.63 & 36,131 & 62,145 & 1.72 & 325 & 146 & .45 & 470 & 602 & 1.28 & 7,116 & 6,262 & .88 & 170,624 & 208,161 & 1.22 \\
\hline 1994 & 2,223 & 3,201 & 1.44 & 37,060 & 68,932 & 1.86 & 535 & 514 & .96 & 735 & 1,029 & 1.40 & 6,344 & 7,803 & 1.23 & 171,191 & 256,787 & 1.50 \\
\hline 1995 & 2,243 & 3,409 & 1.52 & 38,254 & 66,179 & 1.73 & 210 & 271 & 1.29 & 230 & 350 & 1.52 & 5,989 & 5,749 & .96 & 166,383 & 246,247 & 1.48 \\
\hline 1996 & 1,925 & 2,464 & 1.28 & 42,659 & 61,856 & 1.45 & 270 & 348 & 1.29 & 325 & 198 & .61 & 3,280 & 3,870 & 1.18 & 163,841 & 214,632 & 1.31 \\
\hline 1997 & 2,220 & 3,064 & 1.38 & 53,421 & 75,858 & 1.42 & 1,458 & 1,108 & .76 & 425 & 531 & 1.25 & 2,083 & 1,458 & .70 & 153,809 & 184,571 & 1.20 \\
\hline 1998 & 2,523 & 3,785 & 1.50 & 59,138 & 88,116 & 1.49 & 0 & 0 & 0 & 1,005 & 1,417 & 1.41 & 2,280 & 1,915 & .84 & 149,463 & 164,409 & 1.10 \\
\hline 1999 & 2,037 & 2,913 & 1.43 & 59,992 & 79,189 & 1.32 & 124 & 100 & .81 & 2,444 & 2,591 & 1.06 & 2,693 & 1,912 & .71 & 145,584 & 144,128 & .99 \\
\hline 2000 & 1,934 & 3,249 & 1.68 & 62,158 & 99,453 & 1.60 & 0 & 0 & 0 & 3,396 & 4,415 & 1.30 & 2,977 & 2,382 & .80 & 139,650 & 166,184 & 1.19 \\
\hline 2001 & 2,490 & 3,461 & 1.39 & 60,180 & 85,456 & 1.42 & 0 & 0 & 0 & 3,118 & 4,459 & 1.43 & 1,982 & 1,368 & .69 & 132,710 & 145,981 & 1.10 \\
\hline 2002 & 5,981 & 10,168 & 1.70 & 56,574 & 95,044 & 1.68 & 1,376 & 1,459 & 1.06 & 1,424 & 2,278 & 1.60 & 1,328 & 1,049 & .79 & 146,718 & 193,668 & 1.32 \\
\hline 2003 & 6,353 & 11,054 & 1.74 & 48,045 & 72,068 & 1.50 & 1,979 & 2,870 & 1.45 & 1,903 & 2,303 & 1.21 & 1,928 & 1,832 & .95 & 139,745 & 159,309 & 1.14 \\
\hline Average & 2,802 & 4,432 & 1.54 & 46,912 & 73,859 & 1.61 & 874 & 1,098 & .83 & 1,259 & 1,648 & 1.29 & 3,811 & 3,734 & .92 & 156,714 & 205,432 & 1.29 \\
\hline \multicolumn{19}{|c|}{ Kearny County } \\
\hline 1991 & 20,647 & 43,565 & 2.11 & 17,282 & 32,317 & 1.87 & 1,736 & 2,899 & 1.67 & 405 & 595 & 1.47 & 4,447 & 4,358 & .98 & 54,061 & 84,335 & 1.56 \\
\hline 1992 & 18,360 & 34,884 & 1.90 & 21,649 & 33,772 & 1.56 & 1,103 & 1,004 & .91 & 259 & 347 & 1.34 & 4,696 & 5,119 & 1.09 & 51,699 & 58,420 & 1.13 \\
\hline 1993 & 18,441 & 34,116 & 1.85 & 24,078 & 37,080 & 1.54 & 1,776 & 1,279 & .72 & 0 & 0 & 0 & 3,953 & 3,202 & .81 & 50,113 & 49,612 & .99 \\
\hline 1994 & 20,325 & 40,447 & 1.99 & 23,535 & 40,716 & 1.73 & 1,175 & 1,575 & 1.34 & 130 & 283 & 2.18 & 2,976 & 2,887 & .97 & 49,730 & 63,654 & 1.28 \\
\hline 1995 & 22,428 & 40,595 & 1.81 & 20,336 & 34,978 & 1.72 & 788 & 630 & .80 & 0 & 0 & 0 & 2,689 & 1,963 & .73 & 49,822 & 51,317 & 1.03 \\
\hline 1996 & 21,517 & 37,224 & 1.73 & 27,406 & 41,657 & 1.52 & 509 & 448 & .88 & 0 & 0 & 0 & 1,691 & 1,048 & .62 & 45,881 & 51,387 & 1.12 \\
\hline 1997 & 24,356 & 43,597 & 1.79 & 30,368 & 43,123 & 1.42 & 275 & 146 & .53 & 0 & 0 & 0 & 1,833 & 990 & .54 & 47,340 & 44,026 & .93 \\
\hline 1998 & 26,995 & 49,131 & 1.82 & 32,017 & 41,622 & 1.30 & 0 & 0 & 0 & 550 & 798 & 1.45 & 1,004 & 562 & .56 & 43,253 & 39,793 & .92 \\
\hline 1999 & 23,254 & 39,299 & 1.69 & 34,932 & 49,603 & 1.42 & 152 & 214 & 1.41 & 380 & 464 & 1.22 & 1,119 & 705 & .63 & 43,213 & 43,645 & 1.01 \\
\hline 2000 & 24,783 & 51,301 & 2.07 & 35,429 & 58,104 & 1.64 & 680 & 932 & 1.37 & 1,133 & 1,835 & 1.62 & 1,941 & 1,863 & .96 & 39,251 & 47,494 & 1.21 \\
\hline 2001 & 28,477 & 51,543 & 1.81 & 24,025 & 31,473 & 1.31 & 510 & 372 & .73 & 1,148 & 1,940 & 1.69 & 4,082 & 2,163 & .53 & 42,869 & 44,584 & 1.04 \\
\hline 2002 & 34,244 & 72,255 & 2.11 & 24,268 & 42,954 & 1.77 & 235 & 362 & 1.54 & 1,326 & 2,254 & 1.70 & 985 & 1,251 & 1.27 & 46,217 & 66,552 & 1.44 \\
\hline 2003 & 32,457 & 61,019 & 1.88 & 20,351 & 30,527 & 1.50 & 695 & 813 & 1.17 & 824 & 1,211 & 1.47 & 1,676 & 922 & .55 & 47,843 & 52,149 & 1.09 \\
\hline Average & 24,330 & 46,075 & 1.89 & 25,821 & 39,840 & 1.56 & 741 & 821 & 1.01 & 473 & 748 & 1.09 & 2,546 & 2,079 & .79 & 47,022 & 53,613 & 1.13 \\
\hline
\end{tabular}


Table 2. Irrigation water use, acres irrigated, and irrigation application rate by crop type for counties in the Groundwater Management Districts within the Kansas High Plains, 1991-2003-Continued

[Data from Kansas Water Office and Kansas Department of Agriculture (1993-2005). acre-ft, acre-foot; acre-ft/acre, acre-foot per acre]

\begin{tabular}{|c|c|c|c|c|c|c|c|c|c|c|c|c|c|c|c|c|c|c|}
\hline \multirow[b]{2}{*}{ Year } & \multicolumn{3}{|c|}{ Alfalfa } & \multicolumn{3}{|c|}{ Corn } & \multicolumn{3}{|c|}{ Grain sorghum } & \multicolumn{3}{|c|}{ Soybeans } & \multicolumn{3}{|c|}{ Wheat } & \multicolumn{3}{|c|}{ Other/Multiple use } \\
\hline & $\begin{array}{c}\text { Total } \\
\text { acres } \\
\text { irrigated }\end{array}$ & $\begin{array}{l}\text { Irriga- } \\
\text { tion } \\
\text { water } \\
\text { used } \\
\text { (acre-ft) }\end{array}$ & $\begin{array}{l}\text { Rate of } \\
\text { irri- } \\
\text { gation } \\
\text { (acre- } \\
\text { ft/acre) }\end{array}$ & $\begin{array}{c}\text { Total } \\
\text { acres } \\
\text { irrigated }\end{array}$ & $\begin{array}{l}\text { Irriga- } \\
\text { tion } \\
\text { water } \\
\text { used } \\
\text { (acre-ft) }\end{array}$ & $\begin{array}{l}\text { Rate of } \\
\text { irri- } \\
\text { gation } \\
\text { (acre- } \\
\text { ft/acre) }\end{array}$ & $\begin{array}{c}\text { Total } \\
\text { acres } \\
\text { irrigated }\end{array}$ & $\begin{array}{l}\text { Irriga- } \\
\text { tion } \\
\text { water } \\
\text { used } \\
\text { (acre-ft) }\end{array}$ & $\begin{array}{l}\text { Rate of } \\
\text { irri- } \\
\text { gation } \\
\text { (acre- } \\
\text { ft/acre) }\end{array}$ & $\begin{array}{c}\text { Total } \\
\text { acres } \\
\text { irrigated }\end{array}$ & $\begin{array}{l}\text { Irriga- } \\
\text { tion } \\
\text { water } \\
\text { used } \\
\text { (acre-ft) }\end{array}$ & $\begin{array}{l}\text { Rate of } \\
\text { irri- } \\
\text { gation } \\
\text { (acre- } \\
\text { ft/acre) }\end{array}$ & $\begin{array}{c}\text { Total } \\
\text { acres } \\
\text { irrigated }\end{array}$ & $\begin{array}{l}\text { Irriga- } \\
\text { tion } \\
\text { water } \\
\text { used } \\
\text { (acre-ft) }\end{array}$ & $\begin{array}{l}\text { Rate of } \\
\text { irri- } \\
\text { gation } \\
\text { (acre- } \\
\text { ft/acre) }\end{array}$ & $\begin{array}{c}\text { Total } \\
\text { acres } \\
\text { irrigated }\end{array}$ & $\begin{array}{l}\text { Irriga- } \\
\text { tion } \\
\text { water } \\
\text { used } \\
\text { (acre-ft) }\end{array}$ & $\begin{array}{c}\text { Rate of } \\
\text { irri- } \\
\text { gation } \\
\text { (acre- } \\
\text { ft/acre) }\end{array}$ \\
\hline \multicolumn{19}{|c|}{ Kiowa County } \\
\hline 1991 & 8,274 & 12,659 & 1.53 & 14,358 & 23,978 & 1.67 & 1,795 & 2,100 & 1.17 & 4,412 & 6,794 & 1.54 & 1,412 & 890 & 0.63 & 18,963 & 28,824 & 1.52 \\
\hline 1992 & 5,735 & 6,079 & 1.06 & 19,562 & 19,758 & 1.01 & 1,202 & 889 & .74 & 3,785 & 3,671 & .97 & 1,202 & 553 & .46 & 17,397 & 16,179 & .93 \\
\hline 1993 & 5,413 & 4,926 & .91 & 19,918 & 23,902 & 1.20 & 687 & 357 & .52 & 4,584 & 5,226 & 1.14 & 844 & 279 & .33 & 17,113 & 17,113 & 10 \\
\hline 1994 & 5,618 & 7,528 & 1.34 & 19,414 & 28,150 & 1.45 & 962 & 875 & .91 & 4,421 & 6,234 & 1.41 & 390 & 133 & .34 & 18,007 & 23,949 & 1.33 \\
\hline 1995 & 4,510 & 4,059 & .90 & 20,000 & 26,000 & 1.30 & 832 & 874 & 1.05 & 3,501 & 4,691 & 1.34 & 1,668 & 634 & .38 & 18,173 & 19,627 & 1.08 \\
\hline 1996 & 4,930 & 4,141 & .84 & 24,118 & 24,600 & 1.02 & 439 & 206 & .47 & 4,510 & 3,563 & .79 & 638 & 236 & .37 & 16,037 & 13,150 & .82 \\
\hline 1997 & 4,175 & 2,964 & .71 & 23,067 & 22,144 & .96 & 537 & 397 & .74 & 7,452 & 6,632 & .89 & 730 & 380 & .52 & 15,404 & 11,861 & .77 \\
\hline 1998 & 3,310 & 4,535 & 1.37 & 23,104 & 33,501 & 1.45 & 130 & 78 & .60 & 9,201 & 12,329 & 1.34 & 990 & 347 & .35 & 15,117 & 18,443 & 1.22 \\
\hline 1999 & 3,779 & 3,817 & 1.01 & 20,562 & 25,291 & 1.23 & 240 & 230 & .96 & 10,197 & 13,154 & 1.29 & 2,064 & 991 & .48 & 15,181 & 16,244 & 1.07 \\
\hline 2000 & 3,733 & 4,666 & 1.25 & 20,862 & 27,121 & 1.30 & 130 & 104 & .80 & 8,431 & 10,792 & 1.28 & 2,250 & 1,508 & .67 & 16,446 & 19,900 & 1.21 \\
\hline 2001 & 4,605 & 6,447 & 1.40 & 20,624 & 30,524 & 1.48 & 528 & 517 & .98 & 7,976 & 11,964 & 1.50 & 1,473 & 737 & .50 & 16,333 & 20,416 & 1.25 \\
\hline 2002 & 5,262 & 7,419 & 1.41 & 24,057 & 34,402 & 1.43 & 922 & 821 & .89 & 7,132 & 9,700 & 1.36 & 899 & 396 & .44 & 14,579 & 16,328 & 1.12 \\
\hline 2003 & 5,122 & 6,351 & 1.24 & 23,924 & 34,211 & 1.43 & 1,682 & 1,732 & 1.03 & 5,531 & 7,633 & 1.38 & 971 & 573 & .59 & 15,399 & 16,939 & 1.10 \\
\hline Average & 4,959 & 5,815 & 1.15 & 21,044 & 27,199 & 1.30 & 776 & 706 & .84 & 6,241 & 7,876 & 1.25 & 1,195 & 589 & .47 & 16,473 & 18,383 & 1.11 \\
\hline \multicolumn{19}{|c|}{ Lane County } \\
\hline 1991 & 98 & 90 & .92 & 2,478 & 4,039 & 1.63 & 517 & 729 & 1.41 & 0 & 0 & 0 & 588 & 612 & 1.04 & 20,746 & 22,198 & 1.07 \\
\hline 1992 & 559 & 347 & .62 & 2,637 & 3,244 & 1.23 & 758 & 561 & .74 & 0 & 0 & 0 & 593 & 362 & .61 & 15,717 & 12,102 & .77 \\
\hline 1993 & 100 & 139 & 1.39 & 4,244 & 4,456 & 1.05 & 210 & 90 & .43 & 0 & 0 & 0 & 409 & 299 & .73 & 12,117 & 6,180 & .51 \\
\hline 1994 & 0 & 0 & 0 & 4,478 & 5,821 & 1.30 & 206 & 173 & .84 & 0 & 0 & 0 & 236 & 184 & .78 & 13,570 & 13,841 & 1.02 \\
\hline 1995 & 0 & 0 & 0 & 5,664 & 6,230 & 1.10 & 392 & 396 & 1.01 & 0 & 0 & 0 & 258 & 289 & 1.12 & 15,391 & 14,160 & .92 \\
\hline 1996 & 123 & 68 & .55 & 7,309 & 6,578 & .90 & 487 & 253 & .52 & 0 & 0 & 0 & 192 & 52 & .27 & 13,119 & 8,396 & .64 \\
\hline 1997 & 120 & 64 & .53 & 5,971 & 4,717 & .79 & 248 & 117 & .47 & 0 & 0 & 0 & 483 & 256 & .53 & 14,623 & 9,797 & .67 \\
\hline 1998 & 120 & 106 & .88 & 5,648 & 5,083 & .90 & 111 & 89 & .80 & 431 & 315 & .73 & 244 & 46 & .19 & 15,741 & 9,445 & .60 \\
\hline 1999 & 196 & 102 & .52 & 4,293 & 3,778 & .88 & 417 & 463 & 1.11 & 32 & 40 & 1.24 & 323 & 152 & .47 & 17,901 & 12,173 & .68 \\
\hline 2000 & 0 & 0 & 0 & 5,572 & 5,182 & .93 & 448 & 327 & .73 & 835 & 785 & .94 & 410 & 209 & .51 & 15,647 & 12,361 & .79 \\
\hline 2001 & 123 & 154 & 1.25 & 2,549 & 2,600 & 1.02 & 733 & 462 & .63 & 544 & 435 & .80 & 54 & 29 & .54 & 15,504 & 13,178 & .85 \\
\hline 2002 & 248 & 337 & 1.36 & 1,499 & 2,189 & 1.46 & 797 & 948 & 1.19 & 546 & 519 & .95 & 244 & 271 & 1.11 & 15,631 & 14,224 & .91 \\
\hline 2003 & 120 & 188 & 1.57 & 1,775 & 2,112 & 1.19 & 400 & 372 & .93 & 208 & 154 & .74 & 1,010 & 525 & .52 & 16,624 & 17,621 & 1.06 \\
\hline Average & 139 & 123 & .74 & 4,163 & 4,310 & 1.11 & 440 & 383 & .83 & 200 & 173 & .42 & 388 & 253 & .65 & 15,564 & 12,744 & .81 \\
\hline
\end{tabular}


Table 2. Irrigation water use, acres irrigated, and irrigation application rate by crop type for counties in the Groundwater Management Districts within the Kansas High Plains, 1991-2003-Continued

[Data from Kansas Water Office and Kansas Department of Agriculture (1993-2005). acre-ft, acre-foot; acre-ft/acre, acre-foot per acre]

\begin{tabular}{|c|c|c|c|c|c|c|c|c|c|c|c|c|c|c|c|c|c|c|}
\hline \multirow[b]{2}{*}{ Year } & \multicolumn{3}{|c|}{ Alfalfa } & \multicolumn{3}{|c|}{ Corn } & \multicolumn{3}{|c|}{ Grain sorghum } & \multicolumn{3}{|c|}{ Soybeans } & \multicolumn{3}{|c|}{ Wheat } & \multicolumn{3}{|c|}{ Other/Multiple use } \\
\hline & $\begin{array}{c}\text { Total } \\
\text { acres } \\
\text { irrigated }\end{array}$ & $\begin{array}{l}\text { Irriga- } \\
\text { tion } \\
\text { water } \\
\text { used } \\
\text { (acre-ft) }\end{array}$ & $\begin{array}{l}\text { Rate of } \\
\text { irri- } \\
\text { gation } \\
\text { (acre- } \\
\text { ft/acre) }\end{array}$ & $\begin{array}{c}\text { Total } \\
\text { acres } \\
\text { irrigated }\end{array}$ & $\begin{array}{l}\text { Irriga- } \\
\text { tion } \\
\text { water } \\
\text { used } \\
\text { (acre-ft) }\end{array}$ & $\begin{array}{l}\text { Rate of } \\
\text { irri- } \\
\text { gation } \\
\text { (acre- } \\
\text { ft/acre) }\end{array}$ & $\begin{array}{c}\text { Total } \\
\text { acres } \\
\text { irrigated }\end{array}$ & $\begin{array}{l}\text { Irriga- } \\
\text { tion } \\
\text { water } \\
\text { used } \\
\text { (acre-ft) }\end{array}$ & $\begin{array}{l}\text { Rate of } \\
\text { irri- } \\
\text { gation } \\
\text { (acre- } \\
\text { ft/acre) }\end{array}$ & $\begin{array}{c}\text { Total } \\
\text { acres } \\
\text { irrigated }\end{array}$ & $\begin{array}{l}\text { Irriga- } \\
\text { tion } \\
\text { water } \\
\text { used } \\
\text { (acre-ft) }\end{array}$ & $\begin{array}{l}\text { Rate of } \\
\text { irri- } \\
\text { gation } \\
\text { (acre- } \\
\text { ft/acre) }\end{array}$ & $\begin{array}{c}\text { Total } \\
\text { acres } \\
\text { irrigated }\end{array}$ & $\begin{array}{l}\text { Irriga- } \\
\text { tion } \\
\text { water } \\
\text { used } \\
\text { (acre-ft) }\end{array}$ & $\begin{array}{l}\text { Rate of } \\
\text { irri- } \\
\text { gation } \\
\text { (acre- } \\
\text { ft/acre) }\end{array}$ & $\begin{array}{c}\text { Total } \\
\text { acres } \\
\text { irrigated }\end{array}$ & $\begin{array}{l}\text { Irriga- } \\
\text { tion } \\
\text { water } \\
\text { used } \\
\text { (acre-ft) }\end{array}$ & $\begin{array}{l}\text { Rate of } \\
\text { irri- } \\
\text { gation } \\
\text { (acre- } \\
\text { ft/acre) }\end{array}$ \\
\hline \multicolumn{19}{|c|}{ McPherson County } \\
\hline 1991 & 493 & 611 & 1.24 & 5,505 & 7,212 & 1.31 & 1,980 & 1,445 & .73 & 2,859 & 3,088 & 1.08 & 111 & 151 & 1.36 & 19,487 & 24,359 & 1.25 \\
\hline 1992 & 523 & 220 & .42 & 5,622 & 2,249 & .40 & 1,418 & 510 & .36 & 2,812 & 1,097 & .39 & 128 & 64 & .50 & 17,396 & 8,176 & .47 \\
\hline 1993 & 294 & 74 & .25 & 5,451 & 2,671 & .49 & 871 & 174 & .20 & 2,566 & 1,257 & .49 & 138 & 3 & .02 & 15,789 & 8,210 & .52 \\
\hline 1994 & 353 & 219 & .62 & 6,392 & 7,479 & 1.17 & 1,020 & 479 & .47 & 3,051 & 2,959 & .97 & 0 & 0 & 0 & 18,716 & 19,277 & 1.03 \\
\hline 1995 & 403 & 97 & .24 & 5,591 & 5,144 & .92 & 1,079 & 615 & .57 & 3,349 & 2,813 & .84 & 270 & 62 & .23 & 17,525 & 15,072 & .86 \\
\hline 1996 & 196 & 139 & .71 & 7,404 & 6,812 & .92 & 680 & 333 & .49 & 3,658 & 2,231 & .61 & 316 & 44 & .14 & 18,468 & 14,590 & .79 \\
\hline 1997 & 140 & 50 & .36 & 7,075 & 5,306 & .75 & 851 & 408 & .48 & 5,863 & 3,811 & .65 & 316 & 28 & .09 & 17,210 & 12,563 & .73 \\
\hline 1998 & 180 & 74 & .41 & 8,718 & 6,887 & .79 & 945 & 473 & .50 & 5,863 & 5,453 & .93 & 318 & 146 & .46 & 16,700 & 14,362 & .86 \\
\hline 1999 & 288 & 271 & .94 & 7,513 & 4,207 & .56 & 339 & 264 & .78 & 7,416 & 4,969 & .67 & 143 & 31 & .22 & 16,862 & 10,623 & .63 \\
\hline 2000 & 224 & 105 & .47 & 7,476 & 5,607 & .75 & 493 & 424 & .86 & 6,966 & 6,269 & .90 & 143 & 19 & .13 & 17,786 & 16,363 & .92 \\
\hline 2001 & 187 & 101 & .54 & 7,491 & 7,116 & .95 & 610 & 482 & .79 & 6,987 & 7,616 & 1.09 & 393 & 67 & .17 & 17,601 & 17,777 & 1.01 \\
\hline 2002 & 846 & 601 & .71 & 8,291 & 8,788 & 1.06 & 1,282 & 1,231 & .96 & 7,168 & 7,455 & 1.04 & 24 & 25 & 1.04 & 16,937 & 17,276 & 1.02 \\
\hline 2003 & 550 & 319 & .58 & 11,090 & 12,421 & 1.12 & 948 & 853 & .90 & 6,007 & 8,049 & 1.34 & 0 & 0 & 0 & 15,393 & 17,394 & 1.13 \\
\hline Average & 360 & 222 & .58 & 7,201 & 6,300 & .86 & 963 & 592 & .62 & 4,967 & 4,390 & .85 & 177 & 49 & .34 & 17,375 & 15,080 & .86 \\
\hline \multicolumn{19}{|c|}{ Meade County } \\
\hline 1991 & 685 & 795 & 1.16 & 9,910 & 19,424 & 1.96 & 5,089 & 9,466 & 1.86 & 260 & 351 & 1.35 & 3,469 & 4,857 & 1.40 & 105,371 & 181,238 & 1.72 \\
\hline 1992 & 397 & 202 & .51 & 17,480 & 25,870 & 1.48 & 4,036 & 4,722 & 1.17 & 355 & 277 & .78 & 3,364 & 2,658 & .79 & 97,920 & 135,130 & 1.38 \\
\hline 1993 & 834 & 892 & 1.07 & 20,583 & 33,139 & 1.61 & 1,925 & 1,867 & .97 & 250 & 103 & .41 & 2,379 & 1,808 & .76 & 98,471 & 124,073 & 1.26 \\
\hline 1994 & 1,390 & 1,432 & 1.03 & 28,763 & 48,034 & 1.67 & 890 & 1,068 & 1.20 & 0 & 0 & 0 & 1,985 & 1,429 & .72 & 89,818 & 130,236 & 1.45 \\
\hline 1995 & 1,997 & 2,736 & 1.37 & 22,521 & 38,736 & 1.72 & 2,356 & 2,285 & .97 & 0 & 0 & 0 & 1,070 & 1,380 & 1.29 & 91,310 & 128,747 & 1.41 \\
\hline 1996 & 1,660 & 1,710 & 1.03 & 26,951 & 42,044 & 1.56 & 1,935 & 2,032 & 1.05 & 205 & 176 & .86 & 1,025 & 646 & .63 & 93,017 & 119,992 & 1.29 \\
\hline 1997 & 2,320 & 1,879 & .81 & 30,012 & 41,417 & 1.38 & 2,020 & 1,959 & .97 & 505 & 833 & 1.65 & 1,188 & 190 & .16 & 88,354 & 98,073 & 1.11 \\
\hline 1998 & 2,466 & 2,984 & 1.21 & 39,537 & 63,655 & 1.61 & 835 & 443 & .53 & 485 & 475 & .98 & 1,332 & 1,678 & 1.26 & 80,071 & 101,690 & 1.27 \\
\hline 1999 & 1,861 & 1,991 & 1.07 & 43,267 & 55,382 & 1.28 & 177 & 156 & .88 & 1,059 & 1,387 & 1.31 & 1,958 & 1,547 & .79 & 78,498 & 81,638 & 1.04 \\
\hline 2000 & 2,452 & 3,335 & 1.36 & 46,343 & 72,295 & 1.56 & 1,165 & 1,433 & 1.23 & 788 & 1,174 & 1.49 & 1,777 & 1,670 & .94 & 75,811 & 100,071 & 1.32 \\
\hline 2001 & 2,842 & 3,666 & 1.29 & 43,666 & 66,809 & 1.53 & 880 & 933 & 1.06 & 1,352 & 2,339 & 1.73 & 2,062 & 2,103 & 1.02 & 68,185 & 84,549 & 1.24 \\
\hline 2002 & 2,593 & 4,071 & 1.57 & 43,450 & 80,817 & 1.86 & 1,382 & 1,520 & 1.10 & 744 & 1,354 & 1.82 & 1,779 & 1,388 & .78 & 75,942 & 112,394 & 1.48 \\
\hline 2003 & 3,147 & 3,147 & 10 & 42,141 & 65,319 & 1.55 & 1,929 & 1,659 & .86 & 1,587 & 1,841 & 1.16 & 2,486 & 1,666 & .67 & 70,939 & 82,999 & 1.17 \\
\hline Average & 1,896 & 2,218 & 1.11 & 31,894 & 50,226 & 1.60 & 1,894 & 2,273 & 1.07 & 584 & 793 & 1.04 & 1,990 & 1,771 & .86 & 85,670 & 113,910 & 1.32 \\
\hline
\end{tabular}


Table 2. Irrigation water use, acres irrigated, and irrigation application rate by crop type for counties in the Groundwater Management Districts within the Kansas High Plains, 1991-2003-Continued

[Data from Kansas Water Office and Kansas Department of Agriculture (1993-2005). acre-ft, acre-foot; acre-ft/acre, acre-foot per acre]

\begin{tabular}{|c|c|c|c|c|c|c|c|c|c|c|c|c|c|c|c|c|c|c|}
\hline \multirow[b]{2}{*}{ Year } & \multicolumn{3}{|c|}{ Alfalfa } & \multicolumn{3}{|c|}{ Corn } & \multicolumn{3}{|c|}{ Grain sorghum } & \multicolumn{3}{|c|}{ Soybeans } & \multicolumn{3}{|c|}{ Wheat } & \multicolumn{3}{|c|}{ Other/Multiple use } \\
\hline & $\begin{array}{c}\text { Total } \\
\text { acres } \\
\text { irrigated }\end{array}$ & $\begin{array}{l}\text { Irriga- } \\
\text { tion } \\
\text { water } \\
\text { used } \\
\text { (acre-ft) }\end{array}$ & $\begin{array}{c}\text { Rate of } \\
\text { irri- } \\
\text { gation } \\
\text { (acre- } \\
\text { ft/acre) }\end{array}$ & $\begin{array}{c}\text { Total } \\
\text { acres } \\
\text { irrigated }\end{array}$ & $\begin{array}{c}\text { Irriga- } \\
\text { tion } \\
\text { water } \\
\text { used } \\
\text { (acre-ft) }\end{array}$ & $\begin{array}{l}\text { Rate of } \\
\text { irri- } \\
\text { gation } \\
\text { (acre- } \\
\text { ft/acre) }\end{array}$ & $\begin{array}{c}\text { Total } \\
\text { acres } \\
\text { irrigated }\end{array}$ & $\begin{array}{l}\text { Irriga- } \\
\text { tion } \\
\text { water } \\
\text { used } \\
\text { (acre-ft) }\end{array}$ & $\begin{array}{c}\text { Rate of } \\
\text { irri- } \\
\text { gation } \\
\text { (acre- } \\
\mathrm{ft} / \text { acre) }\end{array}$ & $\begin{array}{c}\text { Total } \\
\text { acres } \\
\text { irrigated }\end{array}$ & $\begin{array}{c}\text { Irriga- } \\
\text { tion } \\
\text { water } \\
\text { used } \\
\text { (acre-ft) }\end{array}$ & $\begin{array}{c}\text { Rate of } \\
\text { irri- } \\
\text { gation } \\
\text { (acre- } \\
\text { ft/acre) }\end{array}$ & $\begin{array}{c}\text { Total } \\
\text { acres } \\
\text { irrigated }\end{array}$ & $\begin{array}{l}\text { Irriga- } \\
\text { tion } \\
\text { water } \\
\text { used } \\
\text { (acre-ft) }\end{array}$ & $\begin{array}{l}\text { Rate of } \\
\text { irri- } \\
\text { gation } \\
\text { (acre- } \\
\text { ft/acre) }\end{array}$ & $\begin{array}{c}\text { Total } \\
\text { acres } \\
\text { irrigated }\end{array}$ & $\begin{array}{c}\text { Irriga- } \\
\text { tion } \\
\text { water } \\
\text { used } \\
\text { (acre-ft) }\end{array}$ & $\begin{array}{c}\text { Rate of } \\
\text { irri- } \\
\text { gation } \\
\text { (acre- } \\
\text { ft/acre) }\end{array}$ \\
\hline \multicolumn{19}{|c|}{ Morton County } \\
\hline 1991 & 2,017 & 4,135 & 2.05 & 1,544 & 2,964 & 1.92 & 1,449 & 2,029 & 1.40 & 0 & 0 & 0 & 1,847 & 2,235 & 1.21 & 43,921 & 72,470 & 1.65 \\
\hline 1992 & 1,279 & 2,468 & 1.93 & 3,266 & 5,422 & 1.66 & 1,674 & 2,411 & 1.44 & 0 & 0 & 0 & 1,427 & 1,969 & 1.38 & 42,002 & 61,743 & 1.47 \\
\hline 1993 & 1,593 & 2,788 & 1.75 & 2,634 & 3,424 & 1.30 & 313 & 291 & .93 & 0 & 0 & 0 & 1,158 & 648 & .56 & 47,327 & 60,105 & 1.27 \\
\hline 1994 & 1,568 & 2,885 & 1.84 & 2,596 & 3,349 & 1.29 & 947 & 578 & .61 & 0 & 0 & 0 & 1,876 & 1,313 & .70 & 45,136 & 57,774 & 1.28 \\
\hline 1995 & 1,247 & 1,958 & 1.57 & 4,191 & 5,951 & 1.42 & 885 & 770 & .87 & 0 & 0 & 0 & 1,117 & 1,229 & 1.10 & 42,184 & 50,621 & 1.20 \\
\hline 1996 & 981 & 1,334 & 1.36 & 3,050 & 4,667 & 1.53 & 1,490 & 581 & .39 & 0 & 0 & 0 & 1,721 & 1,790 & 1.04 & 44,966 & 47,664 & 1.06 \\
\hline 1997 & 1,123 & 1,595 & 1.42 & 5,001 & 6,401 & 1.28 & 1,358 & 1,304 & .96 & 0 & 0 & 0 & 1,633 & 1,486 & .91 & 41,850 & 42,687 & 1.02 \\
\hline 1998 & 1,243 & 2,175 & 1.75 & 6,971 & 9,550 & 1.37 & 704 & 704 & 10 & 0 & 0 & 0 & 1,894 & 1,818 & .96 & 39,112 & 40,285 & 1.03 \\
\hline 1999 & 1,044 & 1,691 & 1.62 & 8,040 & 9,326 & 1.16 & 720 & 518 & .72 & 45 & 48 & 1.07 & 2,106 & 1,474 & .70 & 40,505 & 35,239 & .87 \\
\hline 2000 & 614 & 1,124 & 1.83 & 9,138 & 12,610 & 1.38 & 1,275 & 956 & .75 & 607 & 680 & 1.12 & 3,057 & 2,782 & .91 & 35,856 & 37,290 & 1.04 \\
\hline 2001 & 915 & 1,812 & 1.98 & 7,981 & 8,220 & 1.03 & 1,393 & 919 & .66 & 113 & 96 & .85 & 4,469 & 3,799 & .85 & 32,715 & 29,444 & .90 \\
\hline 2002 & 364 & 535 & 1.47 & 7,440 & 9,821 & 1.32 & 1,621 & 1,459 & .90 & 0 & 0 & 0 & 4,407 & 4,143 & .94 & 28,574 & 34,003 & 1.19 \\
\hline 2003 & 303 & 648 & 2.14 & 6,373 & 6,500 & 1.02 & 1,997 & 1,058 & .53 & 0 & 0 & 0 & 4,023 & 2,414 & .60 & 30,090 & 28,886 & .96 \\
\hline Average & 1,099 & 1,934 & 1.75 & 5,248 & 6,785 & 1.36 & 1,217 & 1,044 & .86 & 59 & 63 & .23 & 2,364 & 2,085 & .91 & 39,557 & 46,016 & 1.15 \\
\hline \multicolumn{19}{|c|}{ Pawnee County } \\
\hline 1991 & 9,150 & 12,810 & 1.40 & 11,623 & 16,040 & 1.38 & 5,499 & 6,819 & 1.24 & 4,507 & 6,039 & 1.34 & 2,469 & 2,099 & .85 & 43,837 & 63,125 & 1.44 \\
\hline 1992 & 8,854 & 7,614 & .86 & 14,797 & 10,950 & .74 & 6,055 & 2,604 & .43 & 3,568 & 2,391 & .67 & 4,982 & 2,939 & .59 & 32,280 & 19,045 & .59 \\
\hline 1993 & 9,477 & 5,118 & .54 & 17,720 & 10,100 & .57 & 4,866 & 1,557 & .32 & 4,061 & 1,827 & .45 & 3,161 & 1,075 & .34 & 25,069 & 11,532 & .46 \\
\hline 1994 & 9,303 & 12,745 & 1.37 & 18,441 & 24,158 & 1.31 & 4,211 & 4,127 & .98 & 4,386 & 5,483 & 1.25 & 2,766 & 1,909 & .69 & 35,415 & 42,144 & 1.19 \\
\hline 1995 & 8,860 & 8,151 & .92 & 19,554 & 22,878 & 1.17 & 6,028 & 4,220 & .70 & 4,746 & 4,366 & .92 & 2,378 & 927 & .39 & 36,010 & 34,570 & .96 \\
\hline 1996 & 9,210 & 6,908 & .75 & 18,939 & 15,719 & .83 & 6,156 & 3,817 & .62 & 4,696 & 3,475 & .74 & 1,495 & 568 & .38 & 33,876 & 22,697 & .67 \\
\hline 1997 & 8,053 & 6,120 & .76 & 17,284 & 13,309 & .77 & 4,499 & 2,429 & .54 & 8,161 & 6,039 & .74 & 1,092 & 491 & .45 & 36,414 & 25,126 & .69 \\
\hline 1998 & 9,461 & 10,123 & 1.07 & 20,741 & 21,363 & 1.03 & 3,119 & 1,653 & .53 & 7,574 & 6,817 & .90 & 649 & 487 & .75 & 39,056 & 37,103 & .95 \\
\hline 1999 & 9,337 & 8,030 & .86 & 22,017 & 18,274 & .83 & 1,838 & 1,507 & .82 & 9,271 & 8,158 & .88 & 1,079 & 550 & .51 & 36,593 & 31,104 & .85 \\
\hline 2000 & 8,771 & 10,262 & 1.17 & 21,894 & 23,208 & 1.06 & 1,919 & 1,401 & .73 & 9,911 & 11,299 & 1.14 & 783 & 180 & .23 & 35,937 & 38,453 & 1.07 \\
\hline 2001 & 8,977 & 10,324 & 1.15 & 21,468 & 22,327 & 1.04 & 2,135 & 1,900 & .89 & 9,736 & 11,002 & 1.13 & 1,147 & 963 & .84 & 36,071 & 36,432 & 1.01 \\
\hline 2002 & 11,706 & 14,515 & 1.24 & 22,451 & 29,411 & 1.31 & 2,081 & 2,123 & 1.02 & 9,366 & 11,614 & 1.24 & 1,729 & 1,089 & .63 & 32,179 & 36,040 & 1.12 \\
\hline 2003 & 11,847 & 12,439 & 1.05 & 19,504 & 23,795 & 1.22 & 3,265 & 2,971 & .91 & 8,741 & 11,188 & 1.28 & 3,363 & 1,816 & .54 & 28,192 & 31,011 & 1.10 \\
\hline Average & 9,462 & 9,628 & 1.01 & 18,956 & 19,349 & 1.02 & 3,975 & 2,856 & .75 & 6,825 & 6,900 & .98 & 2,084 & 1,161 & .55 & 34,687 & 32,952 & .93 \\
\hline
\end{tabular}


Table 2. Irrigation water use, acres irrigated, and irrigation application rate by crop type for counties in the Groundwater Management Districts within the Kansas High Plains, 1991-2003-Continued

[Data from Kansas Water Office and Kansas Department of Agriculture (1993-2005). acre-ft, acre-foot; acre-ft/acre, acre-foot per acre]

\begin{tabular}{|c|c|c|c|c|c|c|c|c|c|c|c|c|c|c|c|c|c|c|}
\hline \multirow[b]{2}{*}{ Year } & \multicolumn{3}{|c|}{ Alfalfa } & \multicolumn{3}{|c|}{ Corn } & \multicolumn{3}{|c|}{ Grain sorghum } & \multicolumn{3}{|c|}{ Soybeans } & \multicolumn{3}{|c|}{ Wheat } & \multicolumn{3}{|c|}{ Other/Multiple use } \\
\hline & $\begin{array}{c}\text { Total } \\
\text { acres } \\
\text { irrigated }\end{array}$ & $\begin{array}{l}\text { Irriga- } \\
\text { tion } \\
\text { water } \\
\text { used } \\
\text { (acre-ft) }\end{array}$ & $\begin{array}{l}\text { Rate of } \\
\text { irri- } \\
\text { gation } \\
\text { (acre- } \\
\text { ft/acre) }\end{array}$ & $\begin{array}{c}\text { Total } \\
\text { acres } \\
\text { irrigated }\end{array}$ & $\begin{array}{l}\text { Irriga- } \\
\text { tion } \\
\text { water } \\
\text { used } \\
\text { (acre-ft) }\end{array}$ & $\begin{array}{l}\text { Rate of } \\
\text { irri- } \\
\text { gation } \\
\text { (acre- } \\
\mathrm{ft} / \text { acre) }\end{array}$ & $\begin{array}{c}\text { Total } \\
\text { acres } \\
\text { irrigated }\end{array}$ & $\begin{array}{l}\text { Irriga- } \\
\text { tion } \\
\text { water } \\
\text { used } \\
\text { (acre-ft) }\end{array}$ & $\begin{array}{l}\text { Rate of } \\
\text { irri- } \\
\text { gation } \\
\text { (acre- } \\
\mathrm{ft} / \text { acre) }\end{array}$ & $\begin{array}{c}\text { Total } \\
\text { acres } \\
\text { irrigated }\end{array}$ & $\begin{array}{l}\text { Irriga- } \\
\text { tion } \\
\text { water } \\
\text { used } \\
\text { (acre-ft) }\end{array}$ & $\begin{array}{l}\text { Rate of } \\
\text { irri- } \\
\text { gation } \\
\text { (acre- } \\
\text { ft/acre) }\end{array}$ & $\begin{array}{c}\text { Total } \\
\text { acres } \\
\text { irrigated }\end{array}$ & $\begin{array}{l}\text { Irriga- } \\
\text { tion } \\
\text { water } \\
\text { used } \\
\text { (acre-ft) }\end{array}$ & $\begin{array}{l}\text { Rate of } \\
\text { irri- } \\
\text { gation } \\
\text { (acre- } \\
\text { ft/acre) }\end{array}$ & $\begin{array}{c}\text { Total } \\
\text { acres } \\
\text { irrigated }\end{array}$ & $\begin{array}{l}\text { Irriga- } \\
\text { tion } \\
\text { water } \\
\text { used } \\
\text { (acre-ft) }\end{array}$ & $\begin{array}{l}\text { Rate of } \\
\text { irri- } \\
\text { gation } \\
\text { (acre- } \\
\text { ft/acre) }\end{array}$ \\
\hline \multicolumn{19}{|c|}{ Pratt County } \\
\hline 1991 & 7,019 & 9,335 & 1.33 & 35,052 & 50,475 & 1.44 & 2,821 & 3,272 & 1.16 & 3,858 & 5,208 & 1.35 & 2,642 & 1,506 & 0.57 & 20,264 & 27,964 & 1.38 \\
\hline 1992 & 6,798 & 6,118 & .90 & 38,122 & 35,453 & .93 & 1,568 & 1,223 & .78 & 2,412 & 1,930 & .80 & 1,461 & 774 & .53 & 20,063 & 16,652 & .83 \\
\hline 1993 & 6,613 & 5,621 & .85 & 40,588 & 41,806 & 1.03 & 933 & 634 & .68 & 3,135 & 3,041 & .97 & 1,245 & 448 & .36 & 18,430 & 16,771 & .91 \\
\hline 1994 & 6,901 & 9,730 & 1.41 & 40,673 & 60,603 & 1.49 & 645 & 703 & 1.09 & 4,638 & 6,725 & 1.45 & 1,666 & 1,666 & 10 & 16,675 & 22,845 & 1.37 \\
\hline 1995 & 7,135 & 6,636 & .93 & 38,489 & 48,881 & 1.27 & 163 & 139 & .85 & 4,478 & 5,194 & 1.16 & 1,950 & 839 & .43 & 18,686 & 20,368 & 1.09 \\
\hline 1996 & 5,268 & 4,636 & .88 & 43,696 & 45,007 & 1.03 & 604 & 399 & .66 & 4,897 & 3,673 & .75 & 701 & 379 & .54 & 16,185 & 14,728 & .91 \\
\hline 1997 & 3,746 & 1,910 & .51 & 46,455 & 39,022 & .84 & 369 & 122 & .33 & 6,202 & 4,838 & .78 & 887 & 319 & .36 & 15,472 & 11,759 & .76 \\
\hline 1998 & 3,365 & 3,500 & 1.04 & 45,912 & 58,308 & 1.27 & 255 & 423 & 1.66 & 9,876 & 11,950 & 1.21 & 1,809 & 651 & .36 & 14,088 & 17,469 & 1.24 \\
\hline 1999 & 2,621 & 2,385 & .91 & 48,077 & 58,654 & 1.22 & 747 & 620 & .83 & 10,761 & 12,806 & 1.19 & 2,518 & 1,259 & .50 & 15,509 & 16,905 & 1.09 \\
\hline 2000 & 2,203 & 2,379 & 1.08 & 48,267 & 54,059 & 1.12 & 636 & 623 & .98 & 11,974 & 14,608 & 1.22 & 2,192 & 1,381 & .63 & 16,442 & 18,251 & 1.11 \\
\hline 2001 & 2,873 & 3,361 & 1.17 & 42,999 & 59,769 & 1.39 & 764 & 665 & .87 & 12,290 & 17,821 & 1.45 & 2,195 & 1,098 & .50 & 21,338 & 26,886 & 1.26 \\
\hline 2002 & 4,145 & 5,098 & 1.23 & 42,827 & 54,819 & 1.28 & 508 & 483 & .95 & 10,138 & 12,774 & 1.26 & 2,067 & 1,075 & .52 & 23,922 & 25,118 & 1.05 \\
\hline 2003 & 5,117 & 5,987 & 1.17 & 45,880 & 66,067 & 1.44 & 2,361 & 2,408 & 1.02 & 7,736 & 10,057 & 1.30 & 1,733 & 763 & .44 & 21,364 & 24,996 & 1.17 \\
\hline Average & 4,908 & 5,131 & 1.03 & 42,849 & 51,763 & 1.21 & 952 & 901 & .91 & 7,107 & 8,510 & 1.15 & 1,774 & 935 & .52 & 18,341 & 20,055 & 1.09 \\
\hline \multicolumn{19}{|c|}{ Rawlins County } \\
\hline 1991 & 2,011 & 2,192 & 1.09 & 4,355 & 5,662 & 1.30 & 1,582 & 1,962 & 1.24 & 170 & 83 & .49 & 593 & 551 & .93 & 7,686 & 8,762 & 1.14 \\
\hline 1992 & 2,006 & 1,585 & .79 & 4,082 & 2,612 & .64 & 2,393 & 861 & .36 & 206 & 97 & .47 & 323 & 255 & .79 & 5,743 & 3,905 & .68 \\
\hline 1993 & 2,234 & 1,296 & .58 & 4,993 & 3,795 & .76 & 967 & 367 & .38 & 308 & 92 & .30 & 366 & 183 & .50 & 6,432 & 5,210 & .81 \\
\hline 1994 & 2,310 & 1,756 & .76 & 5,322 & 6,120 & 1.15 & 606 & 351 & .58 & 122 & 95 & .78 & 725 & 312 & .43 & 8,824 & 7,236 & .82 \\
\hline 1995 & 2,591 & 2,202 & .85 & 4,895 & 6,070 & 1.24 & 545 & 480 & .88 & 265 & 183 & .69 & 559 & 492 & .88 & 10,438 & 7,202 & .69 \\
\hline 1996 & 2,055 & 1,582 & .77 & 8,669 & 7,369 & .85 & 556 & 389 & .70 & 0 & 0 & 0 & 631 & 391 & .62 & 5,334 & 3,894 & .73 \\
\hline 1997 & 2,533 & 2,204 & .87 & 9,181 & 11,017 & 1.20 & 471 & 495 & 1.05 & 122 & 106 & .87 & 538 & 420 & .78 & 5,087 & 5,138 & 1.01 \\
\hline 1998 & 2,681 & 2,198 & .82 & 7,536 & 8,139 & 1.08 & 245 & 147 & .60 & 245 & 181 & .74 & 159 & 56 & .35 & 7,038 & 5,490 & .78 \\
\hline 1999 & 2,310 & 1,409 & .61 & 7,069 & 6,503 & .92 & 337 & 189 & .56 & 480 & 274 & .57 & 1,031 & 557 & .54 & 6,061 & 4,061 & .67 \\
\hline 2000 & 2,082 & 2,373 & 1.14 & 7,635 & 10,994 & 1.44 & 19 & 10 & .51 & 161 & 233 & 1.45 & 1,014 & 619 & .61 & 7,348 & 8,891 & 1.21 \\
\hline 2001 & 1,953 & 1,582 & .81 & 5,929 & 6,996 & 1.18 & 445 & 334 & .75 & 758 & 841 & 1.11 & 1,045 & 387 & .37 & 7,865 & 5,899 & .75 \\
\hline 2002 & 2,570 & 2,776 & 1.08 & 5,669 & 7,596 & 1.34 & 440 & 418 & .95 & 1,021 & 1,623 & 1.59 & 717 & 445 & .62 & 8,682 & 10,418 & 1.20 \\
\hline 2003 & 2,508 & 2,433 & .97 & 3,584 & 4,516 & 1.26 & 483 & 290 & .60 & 591 & 786 & 1.33 & 2,035 & 1,323 & .65 & 9,909 & 10,107 & 1.02 \\
\hline Average & 2,296 & 1,968 & .86 & 6,071 & 6,722 & 1.10 & 699 & 484 & .70 & 342 & 354 & .80 & 749 & 461 & .62 & 7,419 & 6,632 & .89 \\
\hline
\end{tabular}


Table 2. Irrigation water use, acres irrigated, and irrigation application rate by crop type for counties in the Groundwater Management Districts within the Kansas High Plains, 1991-2003-Continued

[Data from Kansas Water Office and Kansas Department of Agriculture (1993-2005). acre-ft, acre-foot; acre-ft/acre, acre-foot per acre]

\begin{tabular}{|c|c|c|c|c|c|c|c|c|c|c|c|c|c|c|c|c|c|c|}
\hline \multirow[b]{2}{*}{ Year } & \multicolumn{3}{|c|}{ Alfalfa } & \multicolumn{3}{|c|}{ Corn } & \multicolumn{3}{|c|}{ Grain sorghum } & \multicolumn{3}{|c|}{ Soybeans } & \multicolumn{3}{|c|}{ Wheat } & \multicolumn{3}{|c|}{ Other/Multiple use } \\
\hline & $\begin{array}{c}\text { Total } \\
\text { acres } \\
\text { irrigated }\end{array}$ & $\begin{array}{l}\text { Irriga- } \\
\text { tion } \\
\text { water } \\
\text { used } \\
\text { (acre-ft) }\end{array}$ & $\begin{array}{c}\text { Rate of } \\
\text { irri- } \\
\text { gation } \\
\text { (acre- } \\
\text { ft/acre) }\end{array}$ & $\begin{array}{c}\text { Total } \\
\text { acres } \\
\text { irrigated }\end{array}$ & $\begin{array}{c}\text { Irriga- } \\
\text { tion } \\
\text { water } \\
\text { used } \\
\text { (acre-ft) }\end{array}$ & $\begin{array}{c}\text { Rate of } \\
\text { irri- } \\
\text { gation } \\
\text { (acre- } \\
\text { ft/acre) }\end{array}$ & $\begin{array}{c}\text { Total } \\
\text { acres } \\
\text { irrigated }\end{array}$ & $\begin{array}{l}\text { Irriga- } \\
\text { tion } \\
\text { water } \\
\text { used } \\
\text { (acre-ft) }\end{array}$ & $\begin{array}{l}\text { Rate of } \\
\text { irri- } \\
\text { gation } \\
\text { (acre- } \\
\mathrm{ft} / \text { acre) }\end{array}$ & $\begin{array}{c}\text { Total } \\
\text { acres } \\
\text { irrigated }\end{array}$ & $\begin{array}{l}\text { Irriga- } \\
\text { tion } \\
\text { water } \\
\text { used } \\
\text { (acre-ft) }\end{array}$ & $\begin{array}{c}\text { Rate of } \\
\text { irri- } \\
\text { gation } \\
\text { (acre- } \\
\text { ft/acre) }\end{array}$ & $\begin{array}{c}\text { Total } \\
\text { acres } \\
\text { irrigated }\end{array}$ & $\begin{array}{c}\text { Irriga- } \\
\text { tion } \\
\text { water } \\
\text { used } \\
\text { (acre-ft) }\end{array}$ & $\begin{array}{c}\text { Rate of } \\
\text { irri- } \\
\text { gation } \\
\text { (acre- } \\
\mathrm{ft} / \text { acre) }\end{array}$ & $\begin{array}{c}\text { Total } \\
\text { acres } \\
\text { irrigated }\end{array}$ & $\begin{array}{c}\text { Irriga- } \\
\text { tion } \\
\text { water } \\
\text { used } \\
\text { (acre-ft) }\end{array}$ & $\begin{array}{c}\text { Rate of } \\
\text { irri- } \\
\text { gation } \\
\text { (acre- } \\
\text { tt/acre) }\end{array}$ \\
\hline \multicolumn{19}{|c|}{ Reno County } \\
\hline 1991 & 1,662 & 2,044 & 1.23 & 11,266 & 14,758 & 1.31 & 3,141 & 2,419 & 0.77 & 2,694 & 3,098 & 1.15 & 485 & 291 & 0.60 & 14,507 & 15,522 & 1.07 \\
\hline 1992 & 1,474 & 811 & .55 & 10,800 & 7,992 & .74 & 3,578 & 1,825 & .51 & 3,034 & 2,184 & .72 & 414 & 128 & .31 & 12,699 & 7,492 & .59 \\
\hline 1993 & 1,470 & 882 & .60 & 11,385 & 8,880 & .78 & 2,819 & 1,494 & .53 & 2,578 & 1,779 & .69 & 753 & 113 & .15 & 12,544 & 7,903 & .63 \\
\hline 1994 & 1,355 & 1,721 & 1.27 & 9,820 & 11,686 & 1.19 & 1,971 & 1,892 & .96 & 3,720 & 4,129 & 1.11 & 477 & 91 & .19 & 15,889 & 15,253 & .96 \\
\hline 1995 & 1,395 & 809 & .58 & 8,112 & 8,680 & 1.07 & 2,439 & 2,073 & .85 & 3,841 & 3,803 & .99 & 296 & 50 & .17 & 17,081 & 14,006 & .82 \\
\hline 1996 & 1,974 & 1,461 & .74 & 11,898 & 12,612 & 1.06 & 2,952 & 1,948 & .66 & 4,613 & 4,152 & .90 & 628 & 220 & .35 & 14,858 & 11,144 & .75 \\
\hline 1997 & 1,788 & 930 & .52 & 12,865 & 10,935 & .85 & 2,103 & 1,073 & .51 & 7,385 & 5,686 & .77 & 825 & 281 & .34 & 13,195 & 8,709 & .66 \\
\hline 1998 & 1,870 & 1,739 & .93 & 13,194 & 14,777 & 1.12 & 1,667 & 1,367 & .82 & 9,519 & 11,613 & 1.22 & 615 & 178 & .29 & 13,398 & 11,656 & .87 \\
\hline 1999 & 1,650 & 1,320 & .80 & 15,085 & 14,632 & .97 & 1,704 & 1,005 & .59 & 12,751 & 12,113 & .95 & 547 & 159 & .29 & 12,564 & 9,046 & .72 \\
\hline 2000 & 1,019 & 1,202 & 1.18 & 14,224 & 15,362 & 1.08 & 1,201 & 925 & .77 & 15,746 & 19,053 & 1.21 & 506 & 207 & .41 & 11,936 & 11,936 & 10 \\
\hline 2001 & 1,515 & 1,364 & .90 & 16,207 & 18,800 & 1.16 & 1,415 & 1,330 & .94 & 12,546 & 15,808 & 1.26 & 857 & 583 & .68 & 13,585 & 15,759 & 1.16 \\
\hline 2002 & 1,361 & 1,429 & 1.05 & 15,964 & 17,720 & 1.11 & 1,938 & 1,492 & .77 & 12,124 & 13,700 & 1.13 & 578 & 249 & .43 & 15,708 & 14,766 & .94 \\
\hline 2003 & 1,751 & 1,348 & .77 & 18,262 & 23,193 & 1.27 & 3,353 & 4,024 & 1.20 & 9,524 & 11,715 & 1.23 & 996 & 797 & .80 & 13,880 & 14,296 & 1.03 \\
\hline Average & 1,560 & 1,312 & .86 & 13,006 & 13,848 & 1.05 & 2,329 & 1,759 & .76 & 7,698 & 8,372 & 1.03 & 614 & 257 & .39 & 13,988 & 12,115 & .86 \\
\hline \multicolumn{19}{|c|}{ Rice County } \\
\hline 1991 & 392 & 561 & 1.43 & 9,598 & 12,861 & 1.34 & 811 & 697 & .86 & 1,583 & 1,884 & 1.19 & 60 & 50 & .83 & 8,738 & 11,359 & 1.30 \\
\hline 1992 & 486 & 214 & .44 & 9,952 & 4,976 & .50 & 1,495 & 583 & .39 & 932 & 457 & .49 & 163 & 52 & .32 & 7,518 & 4,511 & .60 \\
\hline 1993 & 238 & 119 & .50 & 9,845 & 5,218 & .53 & 1,138 & 364 & .32 & 1,183 & 793 & .67 & 590 & 106 & .18 & 6,869 & 3,366 & .49 \\
\hline 1994 & 285 & 1,174 & 4.12 & 9,564 & 11,572 & 1.21 & 1,188 & 1,069 & .90 & 1,217 & 1,387 & 1.14 & 151 & 69 & .46 & 9,045 & 10,673 & 1.18 \\
\hline 1995 & 329 & 184 & .56 & 8,993 & 8,723 & .97 & 1,549 & 867 & .56 & 1,302 & 1,133 & .87 & 132 & 66 & .50 & 8,009 & 6,647 & .83 \\
\hline 1996 & 288 & 112 & .39 & 11,009 & 11,449 & 1.04 & 1,475 & 1,047 & .71 & 961 & 942 & .98 & 162 & 130 & .80 & 7,551 & 5,663 & .75 \\
\hline 1997 & 122 & 93 & .76 & 10,372 & 9,542 & .92 & 940 & 658 & .70 & 2,958 & 2,130 & .72 & 487 & 93 & .19 & 6,873 & 5,223 & .76 \\
\hline 1998 & 516 & 392 & .76 & 10,264 & 8,930 & .87 & 601 & 319 & .53 & 3,480 & 3,236 & .93 & 0 & 0 & 0 & 6,954 & 5,980 & .86 \\
\hline 1999 & 476 & 243 & .51 & 10,165 & 8,945 & .88 & 400 & 276 & .69 & 3,393 & 3,088 & .91 & 59 & 10 & .17 & 8,310 & 7,479 & .90 \\
\hline 2000 & 252 & 161 & .64 & 10,427 & 9,801 & .94 & 360 & 230 & .64 & 3,757 & 4,170 & 1.11 & 166 & 17 & .10 & 7,890 & 8,837 & 1.12 \\
\hline 2001 & 173 & 206 & 1.19 & 10,082 & 12,098 & 1.20 & 284 & 99 & .35 & 4,657 & 6,147 & 1.32 & 0 & 0 & 0 & 8,064 & 9,596 & 1.19 \\
\hline 2002 & 103 & 139 & 1.35 & 9,726 & 10,601 & 1.09 & 1,228 & 995 & .81 & 5,707 & 5,650 & .99 & 209 & 209 & 10 & 6,234 & 5,922 & .95 \\
\hline 2003 & 319 & 354 & 1.11 & 9,444 & 12,844 & 1.36 & 1,442 & 1,182 & .82 & 4,116 & 5,474 & 1.33 & 245 & 167 & .68 & 7,235 & 8,971 & 1.24 \\
\hline Average & 306 & 304 & 1.06 & 9,957 & 9,812 & .99 & 993 & 645 & .64 & 2,711 & 2,807 & .97 & 186 & 74 & .40 & 7,638 & 7,248 & .94 \\
\hline
\end{tabular}


Table 2. Irrigation water use, acres irrigated, and irrigation application rate by crop type for counties in the Groundwater Management Districts within the Kansas High Plains, 1991-2003-Continued

[Data from Kansas Water Office and Kansas Department of Agriculture (1993-2005). acre-ft, acre-foot; acre-ft/acre, acre-foot per acre]

\begin{tabular}{|c|c|c|c|c|c|c|c|c|c|c|c|c|c|c|c|c|c|c|}
\hline \multirow[b]{2}{*}{ Year } & \multicolumn{3}{|c|}{ Alfalfa } & \multicolumn{3}{|c|}{ Corn } & \multicolumn{3}{|c|}{ Grain sorghum } & \multicolumn{3}{|c|}{ Soybeans } & \multicolumn{3}{|c|}{ Wheat } & \multicolumn{3}{|c|}{ Other/Multiple use } \\
\hline & $\begin{array}{c}\text { Total } \\
\text { acres } \\
\text { irrigated }\end{array}$ & $\begin{array}{l}\text { Irriga- } \\
\text { tion } \\
\text { water } \\
\text { used } \\
\text { (acre-ft) }\end{array}$ & $\begin{array}{l}\text { Rate of } \\
\text { irri- } \\
\text { gation } \\
\text { (acre- } \\
\text { ft/acre) }\end{array}$ & $\begin{array}{c}\text { Total } \\
\text { acres } \\
\text { irrigated }\end{array}$ & $\begin{array}{l}\text { Irriga- } \\
\text { tion } \\
\text { water } \\
\text { used } \\
\text { (acre-ft) }\end{array}$ & $\begin{array}{l}\text { Rate of } \\
\text { irri- } \\
\text { gation } \\
\text { (acre- } \\
\mathrm{ft} / \text { acre) }\end{array}$ & $\begin{array}{c}\text { Total } \\
\text { acres } \\
\text { irrigated }\end{array}$ & $\begin{array}{l}\text { Irriga- } \\
\text { tion } \\
\text { water } \\
\text { used } \\
\text { (acre-ft) }\end{array}$ & $\begin{array}{l}\text { Rate of } \\
\text { irri- } \\
\text { gation } \\
\text { (acre- } \\
\text { ft/acre) }\end{array}$ & $\begin{array}{c}\text { Total } \\
\text { acres } \\
\text { irrigated }\end{array}$ & $\begin{array}{l}\text { Irriga- } \\
\text { tion } \\
\text { water } \\
\text { used } \\
\text { (acre-ft) }\end{array}$ & $\begin{array}{l}\text { Rate of } \\
\text { irri- } \\
\text { gation } \\
\text { (acre- } \\
\text { ft/acre) }\end{array}$ & $\begin{array}{c}\text { Total } \\
\text { acres } \\
\text { irrigated }\end{array}$ & $\begin{array}{l}\text { Irriga- } \\
\text { tion } \\
\text { water } \\
\text { used } \\
\text { (acre-ft) }\end{array}$ & $\begin{array}{l}\text { Rate of } \\
\text { irri- } \\
\text { gation } \\
\text { (acre- } \\
\text { ft/acre) }\end{array}$ & $\begin{array}{c}\text { Total } \\
\text { acres } \\
\text { irrigated }\end{array}$ & $\begin{array}{l}\text { Irriga- } \\
\text { tion } \\
\text { water } \\
\text { used } \\
\text { (acre-ft) }\end{array}$ & $\begin{array}{l}\text { Rate of } \\
\text { irri- } \\
\text { gation } \\
\text { (acre- } \\
\text { ft/acre) }\end{array}$ \\
\hline \multicolumn{19}{|c|}{ Scott County } \\
\hline 1991 & 1,617 & 1,827 & 1.13 & 7,964 & 10,672 & 1.34 & 9,776 & 11,145 & 1.14 & 132 & 120 & .91 & 963 & 1,117 & 1.16 & 46,285 & 64,336 & 1.39 \\
\hline 1992 & 909 & 1,600 & 1.76 & 9,253 & 12,121 & 1.31 & 8,027 & 6,903 & .86 & 590 & 749 & 1.27 & 1,676 & 1,592 & .95 & 40,346 & 45,188 & 1.12 \\
\hline 1993 & 604 & 821 & 1.36 & 11,111 & 12,667 & 1.14 & 4,683 & 2,763 & .59 & 67 & 17 & .26 & 729 & 532 & .73 & 35,097 & 28,429 & .81 \\
\hline 1994 & 844 & 1,097 & 1.30 & 7,315 & 10,314 & 1.41 & 4,936 & 3,702 & .75 & 77 & 221 & 2.87 & 707 & 509 & .72 & 44,937 & 53,026 & 1.18 \\
\hline 1995 & 702 & 835 & 1.19 & 8,269 & 10,832 & 1.31 & 4,723 & 3,731 & .79 & 0 & 0 & 0 & 1,625 & 1,349 & .83 & 45,987 & 49,666 & 1.08 \\
\hline 1996 & 805 & 668 & .83 & 11,235 & 13,145 & 1.17 & 3,870 & 2,593 & .67 & 0 & 0 & 0 & 1,065 & 138 & .13 & 42,231 & 36,741 & .87 \\
\hline 1997 & 814 & 700 & .86 & 12,561 & 13,817 & 1.10 & 3,181 & 1,622 & .51 & 58 & 72 & 1.24 & 941 & 640 & .68 & 42,442 & 36,076 & .85 \\
\hline 1998 & 618 & 655 & 1.06 & 13,293 & 15,686 & 1.18 & 1,384 & 747 & .54 & 345 & 290 & .84 & 1,157 & 891 & .77 & 43,923 & 33,821 & .77 \\
\hline 1999 & 525 & 1,150 & 2.19 & 15,527 & 17,080 & 1.10 & 1,988 & 1,491 & .75 & 370 & 466 & 1.26 & 261 & 125 & .48 & 39,321 & 33,030 & .84 \\
\hline 2000 & 435 & 579 & 1.33 & 17,271 & 23,834 & 1.38 & 920 & 764 & .83 & 127 & 118 & .93 & 927 & 473 & .51 & 41,494 & 39,419 & .95 \\
\hline 2001 & 435 & 813 & 1.87 & 14,967 & 17,212 & 1.15 & 1,661 & 947 & .57 & 113 & 104 & .92 & 791 & 506 & .64 & 40,483 & 31,982 & .79 \\
\hline 2002 & 475 & 817 & 1.72 & 12,220 & 19,674 & 1.61 & 2,079 & 2,183 & 1.05 & 214 & 289 & 1.35 & 733 & 520 & .71 & 45,617 & 46,986 & 1.03 \\
\hline 2003 & 435 & 796 & 1.83 & 7,353 & 9,927 & 1.35 & 3,467 & 2,704 & .78 & 228 & 228 & 10 & 1,212 & 812 & .67 & 46,902 & 46,902 & 10 \\
\hline Average & 709 & 951 & 1.42 & 11,411 & 14,383 & 1.27 & 3,900 & 3,177 & .76 & 179 & 206 & .99 & 984 & 708 & .69 & 42,697 & 41,969 & .98 \\
\hline \multicolumn{19}{|c|}{ Sedgwick County } \\
\hline 1991 & 484 & 455 & .94 & 8,393 & 11,079 & 1.32 & 2,012 & 1,771 & .88 & 9,651 & 12,257 & 1.27 & 171 & 96 & .56 & 14,709 & 16,768 & 1.14 \\
\hline 1992 & 743 & 438 & .59 & 7,228 & 5,710 & .79 & 4,008 & 2,445 & .61 & 8,898 & 7,652 & .86 & 379 & 53 & .14 & 11,748 & 9,163 & .78 \\
\hline 1993 & 244 & 351 & 1.44 & 7,385 & 6,277 & .85 & 2,154 & 1,465 & .68 & 9,166 & 8,983 & .98 & 255 & 56 & .22 & 15,175 & 11,381 & .75 \\
\hline 1994 & 60 & 93 & 1.55 & 8,032 & 9,317 & 1.16 & 1,726 & 1,588 & .92 & 10,571 & 11,945 & 1.13 & 321 & 144 & .45 & 14,713 & 14,860 & 1.01 \\
\hline 1995 & 344 & 275 & .80 & 8,607 & 8,177 & .95 & 2,413 & 1,279 & .53 & 9,463 & 8,327 & .88 & 461 & 212 & .46 & 15,029 & 12,324 & .82 \\
\hline 1996 & 40 & 42 & 1.05 & 9,206 & 9,758 & 1.06 & 2,338 & 1,403 & .60 & 8,707 & 7,836 & .90 & 384 & 165 & .43 & 16,088 & 13,675 & .85 \\
\hline 1997 & 352 & 158 & .45 & 8,709 & 6,880 & .79 & 1,735 & 920 & .53 & 12,302 & 9,103 & .74 & 96 & 81 & .84 & 13,869 & 10,124 & .73 \\
\hline 1998 & 200 & 148 & .74 & 8,388 & 8,807 & 1.05 & 1,505 & 1,069 & .71 & 12,644 & 13,023 & 1.03 & 212 & 148 & .70 & 15,164 & 14,406 & .95 \\
\hline 1999 & 149 & 67 & .45 & 10,071 & 7,755 & .77 & 1,355 & 759 & .56 & 12,274 & 10,678 & .87 & 176 & 134 & .76 & 14,705 & 11,617 & .79 \\
\hline 2000 & 204 & 114 & .56 & 9,945 & 9,050 & .91 & 1,404 & 856 & .61 & 11,431 & 12,460 & 1.09 & 150 & 98 & .65 & 14,950 & 14,502 & .97 \\
\hline 2001 & 289 & 277 & .96 & 10,658 & 12,363 & 1.16 & 870 & 800 & .92 & 12,998 & 14,558 & 1.12 & 102 & 91 & .89 & 13,637 & 14,864 & 1.09 \\
\hline 2002 & 447 & 425 & .95 & 13,248 & 14,175 & 1.07 & 759 & 554 & .73 & 12,573 & 13,327 & 1.06 & 267 & 230 & .86 & 13,949 & 13,670 & .98 \\
\hline 2003 & 78 & 9 & .12 & 13,245 & 14,834 & 1.12 & 1,478 & 1,315 & .89 & 10,405 & 11,966 & 1.15 & 224 & 65 & .29 & 14,002 & 14,562 & 1.04 \\
\hline Average & 280 & 220 & .82 & 9,470 & 9,553 & 10 & 1,827 & 1,248 & .71 & 10,853 & 10,932 & 1.01 & 246 & 121 & .56 & 14,441 & 13,224 & .92 \\
\hline
\end{tabular}


Table 2. Irrigation water use, acres irrigated, and irrigation application rate by crop type for counties in the Groundwater Management Districts within the Kansas High Plains, 1991-2003-Continued

[Data from Kansas Water Office and Kansas Department of Agriculture (1993-2005). acre-ft, acre-foot; acre-ft/acre, acre-foot per acre]

\begin{tabular}{|c|c|c|c|c|c|c|c|c|c|c|c|c|c|c|c|c|c|c|}
\hline \multirow[b]{2}{*}{ Year } & \multicolumn{3}{|c|}{ Alfalfa } & \multicolumn{3}{|c|}{ Corn } & \multicolumn{3}{|c|}{ Grain sorghum } & \multicolumn{3}{|c|}{ Soybeans } & \multicolumn{3}{|c|}{ Wheat } & \multicolumn{3}{|c|}{ Other/Multiple use } \\
\hline & $\begin{array}{c}\text { Total } \\
\text { acres } \\
\text { irrigated }\end{array}$ & $\begin{array}{c}\text { Irriga- } \\
\text { tion } \\
\text { water } \\
\text { used } \\
\text { (acre-ft) }\end{array}$ & $\begin{array}{l}\text { Rate of } \\
\text { irri- } \\
\text { gation } \\
\text { (acre- } \\
\text { ft/acre) }\end{array}$ & $\begin{array}{c}\text { Total } \\
\text { acres } \\
\text { irrigated }\end{array}$ & $\begin{array}{l}\text { Irriga- } \\
\text { tion } \\
\text { water } \\
\text { used } \\
\text { (acre-ft) }\end{array}$ & $\begin{array}{l}\text { Rate of } \\
\text { irri- } \\
\text { gation } \\
\text { (acre- } \\
\text { ft/acre) }\end{array}$ & $\begin{array}{c}\text { Total } \\
\text { acres } \\
\text { irrigated }\end{array}$ & $\begin{array}{l}\text { Irriga- } \\
\text { tion } \\
\text { water } \\
\text { used } \\
\text { (acre-ft) }\end{array}$ & $\begin{array}{l}\text { Rate of } \\
\text { irri- } \\
\text { gation } \\
\text { (acre- } \\
\text { ft/acre) }\end{array}$ & $\begin{array}{c}\text { Total } \\
\text { acres } \\
\text { irrigated }\end{array}$ & $\begin{array}{l}\text { Irriga- } \\
\text { tion } \\
\text { water } \\
\text { used } \\
\text { (acre-ft) }\end{array}$ & $\begin{array}{l}\text { Rate of } \\
\text { irri- } \\
\text { gation } \\
\text { (acre- } \\
\text { ft/acre) }\end{array}$ & $\begin{array}{c}\text { Total } \\
\text { acres } \\
\text { irrigated }\end{array}$ & $\begin{array}{l}\text { Irriga- } \\
\text { tion } \\
\text { water } \\
\text { used } \\
\text { (acre-ft) }\end{array}$ & $\begin{array}{l}\text { Rate of } \\
\text { irri- } \\
\text { gation } \\
\text { (acre- } \\
\text { ft/acre) }\end{array}$ & $\begin{array}{c}\text { Total } \\
\text { acres } \\
\text { irrigated }\end{array}$ & $\begin{array}{l}\text { Irriga- } \\
\text { tion } \\
\text { water } \\
\text { used } \\
\text { (acre-ft) }\end{array}$ & $\begin{array}{c}\text { Rate of } \\
\text { irri- } \\
\text { gation } \\
\text { (acre- } \\
\text { ft/acre) }\end{array}$ \\
\hline \multicolumn{19}{|c|}{ Seward County } \\
\hline 1991 & 7,093 & 18,726 & 2.64 & 6,912 & 14,100 & 2.04 & 3,361 & 4,403 & 1.31 & 126 & 158 & 1.25 & 5,781 & 6,475 & 1.12 & 86,623 & 155,921 & 1.80 \\
\hline 1992 & 5,532 & 13,664 & 2.47 & 10,268 & 17,969 & 1.75 & 1,426 & 1,440 & 1.01 & 0 & 0 & 0 & 5,633 & 6,253 & 1.11 & 86,434 & 134,837 & 1.56 \\
\hline 1993 & 6,204 & 11,664 & 1.88 & 10,575 & 18,718 & 1.77 & 1,930 & 2,567 & 1.33 & 0 & 0 & 0 & 4,433 & 4,477 & 1.01 & 84,723 & 113,529 & 1.34 \\
\hline 1994 & 5,652 & 10,682 & 1.89 & 14,892 & 25,614 & 1.72 & 881 & 696 & .79 & 0 & 0 & 0 & 3,488 & 3,244 & .93 & 84,109 & 125,322 & 1.49 \\
\hline 1995 & 6,367 & 14,135 & 2.22 & 14,458 & 24,723 & 1.71 & 1,332 & 1,372 & 1.03 & 0 & 0 & 0 & 3,000 & 2,580 & .86 & 83,141 & 125,543 & 1.51 \\
\hline 1996 & 7,549 & 15,023 & 1.99 & 20,646 & 36,543 & 1.77 & 3,060 & 3,182 & 1.04 & 205 & 84 & .41 & 2,298 & 2,873 & 1.25 & 74,969 & 107,206 & 1.43 \\
\hline 1997 & 7,914 & 14,008 & 1.77 & 21,361 & 32,255 & 1.51 & 1,902 & 2,111 & 1.11 & 0 & 0 & 0 & 3,130 & 3,443 & 1.10 & 82,275 & 99,553 & 1.21 \\
\hline 1998 & 8,519 & 15,079 & 1.77 & 25,519 & 41,596 & 1.63 & 1,649 & 1,468 & .89 & 821 & 731 & .89 & 1,812 & 1,268 & .70 & 84,897 & 97,632 & 1.15 \\
\hline 1999 & 9,027 & 10,923 & 1.21 & 26,159 & 36,884 & 1.41 & 956 & 908 & .95 & 4,024 & 3,783 & .94 & 1,209 & 822 & .68 & 81,386 & 82,200 & 1.01 \\
\hline 2000 & 7,019 & 10,037 & 1.43 & 27,984 & 45,334 & 1.62 & 890 & 685 & .77 & 3,670 & 5,432 & 1.48 & 1,724 & 1,776 & 1.03 & 80,876 & 97,860 & 1.21 \\
\hline 2001 & 6,936 & 11,167 & 1.61 & 30,964 & 46,756 & 1.51 & 877 & 754 & .86 & 2,087 & 2,859 & 1.37 & 1,546 & 696 & .45 & 83,930 & 94,841 & 1.13 \\
\hline 2002 & 8,679 & 15,188 & 1.75 & 29,245 & 58,782 & 2.01 & 305 & 296 & .97 & 1,702 & 2,774 & 1.63 & 2,196 & 1,449 & .66 & 88,786 & 126,076 & 1.42 \\
\hline 2003 & 8,843 & 12,469 & 1.41 & 23,603 & 37,293 & 1.58 & 1,049 & 808 & .77 & 4,245 & 4,712 & 1.11 & 2,712 & 1,654 & .61 & 77,339 & 90,487 & 1.17 \\
\hline Average & 7,333 & 13,289 & 1.85 & 20,199 & 33,582 & 1.69 & 1,509 & 1,592 & .99 & 1,298 & 1,579 & .70 & 2,997 & 2,847 & .89 & 83,038 & 111,616 & 1.34 \\
\hline \multicolumn{19}{|c|}{ Sheridan County } \\
\hline 1991 & 1,466 & 2,067 & 1.41 & 32,022 & 50,595 & 1.58 & 4,483 & 5,335 & 1.19 & 605 & 962 & 1.59 & 1,455 & 1,019 & .70 & 32,680 & 46,079 & 1.41 \\
\hline 1992 & 938 & 507 & .54 & 35,531 & 31,623 & .89 & 2,559 & 1,356 & .53 & 806 & 524 & .65 & 1,136 & 761 & .67 & 26,099 & 17,747 & .68 \\
\hline 1993 & 1,538 & 677 & .44 & 40,105 & 25,667 & .64 & 1,563 & 750 & .48 & 1,110 & 544 & .49 & 1,127 & 372 & .33 & 21,695 & 9,546 & .44 \\
\hline 1994 & 1,101 & 683 & .62 & 42,504 & 53,130 & 1.25 & 1,744 & 1,500 & .86 & 301 & 403 & 1.34 & 500 & 430 & .86 & 25,589 & 26,613 & 1.04 \\
\hline 1995 & 1,460 & 1,241 & .85 & 39,769 & 52,495 & 1.32 & 2,223 & 2,090 & .94 & 608 & 663 & 1.09 & 1,122 & 449 & .40 & 26,529 & 28,917 & 1.09 \\
\hline 1996 & 1,445 & 1,170 & .81 & 44,289 & 47,389 & 1.07 & 1,934 & 1,431 & .74 & 293 & 384 & 1.31 & 562 & 337 & .60 & 24,845 & 21,615 & .87 \\
\hline 1997 & 1,992 & 1,833 & .92 & 49,432 & 50,915 & 1.03 & 1,739 & 1,165 & .67 & 1,196 & 909 & .76 & 729 & 416 & .57 & 19,953 & 16,561 & .83 \\
\hline 1998 & 1,561 & 1,655 & 1.06 & 52,776 & 53,304 & 1.01 & 1,132 & 1,041 & .92 & 598 & 574 & .96 & 509 & 158 & .31 & 18,300 & 14,274 & .78 \\
\hline 1999 & 1,872 & 1,498 & .80 & 51,600 & 45,408 & .88 & 821 & 608 & .74 & 2,221 & 1,310 & .59 & 240 & 31 & .13 & 18,556 & 13,175 & .71 \\
\hline 2000 & 1,707 & 1,690 & .99 & 54,723 & 74,423 & 1.36 & 814 & 773 & .95 & 1,546 & 1,840 & 1.19 & 510 & 332 & .65 & 17,462 & 18,510 & 1.06 \\
\hline 2001 & 1,508 & 1,342 & .89 & 46,946 & 54,927 & 1.17 & 256 & 143 & .56 & 4,536 & 4,808 & 1.06 & 320 & 61 & .19 & 20,602 & 19,984 & .97 \\
\hline 2002 & 1,477 & 1,684 & 1.14 & 45,600 & 66,120 & 1.45 & 935 & 1,262 & 1.35 & 3,557 & 4,766 & 1.34 & 125 & 23 & .18 & 24,738 & 29,933 & 1.21 \\
\hline 2003 & 1,854 & 2,225 & 1.20 & 34,977 & 50,017 & 1.43 & 1,749 & 1,732 & .99 & 2,988 & 3,944 & 1.32 & 2,496 & 1,248 & .50 & 33,581 & 38,954 & 1.16 \\
\hline Average & 1,532 & 1,405 & .90 & 43,867 & 50,463 & 1.16 & 1,689 & 1,476 & .84 & 1,567 & 1,664 & 1.05 & 833 & 433 & .47 & 23,895 & 23,224 & .94 \\
\hline
\end{tabular}


Table 2. Irrigation water use, acres irrigated, and irrigation application rate by crop type for counties in the Groundwater Management Districts within the Kansas High Plains, 1991-2003-Continued

[Data from Kansas Water Office and Kansas Department of Agriculture (1993-2005). acre-ft, acre-foot; acre-ft/acre, acre-foot per acre]

\begin{tabular}{|c|c|c|c|c|c|c|c|c|c|c|c|c|c|c|c|c|c|c|}
\hline \multirow[b]{2}{*}{ Year } & \multicolumn{3}{|c|}{ Alfalfa } & \multicolumn{3}{|c|}{ Corn } & \multicolumn{3}{|c|}{ Grain sorghum } & \multicolumn{3}{|c|}{ Soybeans } & \multicolumn{3}{|c|}{ Wheat } & \multicolumn{3}{|c|}{ Other/Multiple use } \\
\hline & $\begin{array}{c}\text { Total } \\
\text { acres } \\
\text { irrigated }\end{array}$ & $\begin{array}{l}\text { Irriga- } \\
\text { tion } \\
\text { water } \\
\text { used } \\
\text { (acre-ft) }\end{array}$ & $\begin{array}{l}\text { Rate of } \\
\text { irri- } \\
\text { gation } \\
\text { (acre- } \\
\text { ft/acre) }\end{array}$ & $\begin{array}{c}\text { Total } \\
\text { acres } \\
\text { irrigated }\end{array}$ & $\begin{array}{l}\text { Irriga- } \\
\text { tion } \\
\text { water } \\
\text { used } \\
\text { (acre-ft) }\end{array}$ & $\begin{array}{l}\text { Rate of } \\
\text { irri- } \\
\text { gation } \\
\text { (acre- } \\
\mathrm{ft} / \text { acre) }\end{array}$ & $\begin{array}{c}\text { Total } \\
\text { acres } \\
\text { irrigated }\end{array}$ & $\begin{array}{l}\text { Irriga- } \\
\text { tion } \\
\text { water } \\
\text { used } \\
\text { (acre-ft) }\end{array}$ & $\begin{array}{l}\text { Rate of } \\
\text { irri- } \\
\text { gation } \\
\text { (acre- } \\
\text { ft/acre) }\end{array}$ & $\begin{array}{c}\text { Total } \\
\text { acres } \\
\text { irrigated }\end{array}$ & $\begin{array}{l}\text { Irriga- } \\
\text { tion } \\
\text { water } \\
\text { used } \\
\text { (acre-ft) }\end{array}$ & $\begin{array}{l}\text { Rate of } \\
\text { irri- } \\
\text { gation } \\
\text { (acre- } \\
\text { ft/acre) }\end{array}$ & $\begin{array}{c}\text { Total } \\
\text { acres } \\
\text { irrigated }\end{array}$ & $\begin{array}{l}\text { Irriga- } \\
\text { tion } \\
\text { water } \\
\text { used } \\
\text { (acre-ft) }\end{array}$ & $\begin{array}{l}\text { Rate of } \\
\text { irri- } \\
\text { gation } \\
\text { (acre- } \\
\text { ft/acre) }\end{array}$ & $\begin{array}{c}\text { Total } \\
\text { acres } \\
\text { irrigated }\end{array}$ & $\begin{array}{l}\text { Irriga- } \\
\text { tion } \\
\text { water } \\
\text { used } \\
\text { (acre-ft) }\end{array}$ & $\begin{array}{l}\text { Rate of } \\
\text { irri- } \\
\text { gation } \\
\text { (acre- } \\
\text { ft/acre) }\end{array}$ \\
\hline \multicolumn{19}{|c|}{ Sherman County } \\
\hline 1991 & 2,106 & 3,138 & 1.49 & 24,571 & 34,154 & 1.39 & 5,812 & 7,962 & 1.37 & 1,289 & 1,315 & 1.02 & 7,863 & 6,055 & 0.77 & 65,383 & 92,190 & 1.41 \\
\hline 1992 & 1,184 & 1,409 & 1.19 & 30,821 & 30,513 & .99 & 5,208 & 3,958 & .76 & 1,319 & 712 & .54 & 6,561 & 3,740 & .57 & 58,387 & 39,703 & .68 \\
\hline 1993 & 1,700 & 1,530 & .90 & 31,267 & 29,704 & .95 & 116 & 65 & .56 & 1,140 & 718 & .63 & 8,540 & 4,697 & .55 & 62,606 & 27,547 & .44 \\
\hline 1994 & 1,403 & 1,557 & 1.11 & 36,387 & 45,120 & 1.24 & 385 & 281 & .73 & 473 & 284 & .60 & 6,546 & 3,862 & .59 & 65,262 & 67,872 & 1.04 \\
\hline 1995 & 1,927 & 2,100 & 1.09 & 31,107 & 38,262 & 1.23 & 2,481 & 1,786 & .72 & 365 & 318 & .87 & 9,205 & 4,418 & .48 & 58,666 & 63,946 & 1.09 \\
\hline 1996 & 1,780 & 1,833 & 1.03 & 48,320 & 57,501 & 1.19 & 281 & 180 & .64 & 219 & 230 & 1.05 & 6,883 & 3,579 & .52 & 57,116 & 49,691 & .87 \\
\hline 1997 & 1,945 & 2,529 & 1.30 & 51,009 & 69,882 & 1.37 & 459 & 326 & .71 & 1,739 & 1,948 & 1.12 & 6,977 & 3,837 & .55 & 54,696 & 45,398 & .83 \\
\hline 1998 & 2,906 & 3,516 & 1.21 & 52,390 & 64,440 & 1.23 & 944 & 396 & .42 & 2,508 & 2,383 & .95 & 5,396 & 2,914 & .54 & 52,467 & 40,924 & .78 \\
\hline 1999 & 3,056 & 3,362 & 1.10 & 53,013 & 57,254 & 1.08 & 879 & 563 & .64 & 1,755 & 1,843 & 1.05 & 6,216 & 3,294 & .53 & 49,096 & 34,858 & .71 \\
\hline 2000 & 2,609 & 3,574 & 1.37 & 55,260 & 77,917 & 1.41 & 417 & 334 & .80 & 5,064 & 6,786 & 1.34 & 4,377 & 2,670 & .61 & 47,869 & 50,741 & 1.06 \\
\hline 2001 & 2,929 & 4,276 & 1.46 & 51,427 & 69,941 & 1.36 & 1,994 & 1,336 & .67 & 4,217 & 5,018 & 1.19 & 6,153 & 3,815 & .62 & 47,594 & 46,166 & .97 \\
\hline 2002 & 3,269 & 5,557 & 1.70 & 48,102 & 74,558 & 1.55 & 695 & 862 & 1.24 & 3,838 & 6,409 & 1.67 & 5,059 & 3,339 & .66 & 54,538 & 65,991 & 1.21 \\
\hline 2003 & 3,217 & 4,632 & 1.44 & 39,032 & 61,280 & 1.57 & 1,361 & 1,810 & 1.33 & 2,294 & 3,074 & 1.34 & 7,196 & 4,390 & .61 & 63,503 & 73,663 & 1.16 \\
\hline Average & 2,310 & 3,001 & 1.26 & 42,516 & 54,656 & 1.27 & 1,618 & 1,528 & .81 & 2,017 & 2,387 & 1.03 & 6,690 & 3,893 & .58 & 56,706 & 53,745 & .94 \\
\hline \multicolumn{19}{|c|}{ Stafford County } \\
\hline 1991 & 3,206 & 4,392 & 1.37 & 33,049 & 48,913 & 1.48 & 2,507 & 2,607 & 1.04 & 3,839 & 5,336 & 1.39 & 2,921 & 2,249 & .77 & 32,923 & 45,105 & 1.37 \\
\hline 1992 & 3,744 & 2,583 & .69 & 34,166 & 27,674 & .81 & 2,581 & 1,549 & .60 & 2,602 & 2,342 & .90 & 1,990 & 915 & .46 & 32,242 & 27,406 & .85 \\
\hline 1993 & 3,136 & 1,505 & .48 & 34,819 & 25,070 & .72 & 1,469 & 617 & .42 & 3,028 & 2,271 & .75 & 2,881 & 778 & .27 & 31,156 & 20,251 & .65 \\
\hline 1994 & 2,739 & 3,369 & 1.23 & 37,906 & 52,310 & 1.38 & 915 & 540 & .59 & 2,938 & 4,554 & 1.55 & 828 & 265 & .32 & 33,898 & 45,423 & 1.34 \\
\hline 1995 & 2,833 & 2,266 & .80 & 37,039 & 43,706 & 1.18 & 1,772 & 1,329 & .75 & 3,311 & 3,940 & 1.19 & 1,913 & 1,071 & .56 & 29,773 & 29,773 & 10 \\
\hline 1996 & 3,206 & 2,565 & .80 & 40,741 & 39,926 & .98 & 919 & 414 & .45 & 5,046 & 4,440 & .88 & 1,604 & 481 & .30 & 27,906 & 23,720 & .85 \\
\hline 1997 & 3,432 & 1,956 & .57 & 39,373 & 33,073 & .84 & 562 & 461 & .82 & 9,112 & 7,563 & .83 & 1,721 & 551 & .32 & 25,983 & 18,448 & .71 \\
\hline 1998 & 4,631 & 4,677 & 1.01 & 39,702 & 44,466 & 1.12 & 340 & 214 & .63 & 12,437 & 13,556 & 1.09 & 612 & 337 & .55 & 24,059 & 26,465 & 1.10 \\
\hline 1999 & 4,632 & 3,428 & .74 & 36,279 & 36,642 & 1.01 & 293 & 267 & .91 & 13,502 & 14,717 & 1.09 & 1,297 & 661 & .51 & 26,253 & 24,415 & .93 \\
\hline 2000 & 3,242 & 3,566 & 1.10 & 36,126 & 41,906 & 1.16 & 647 & 705 & 1.09 & 13,195 & 16,494 & 1.25 & 755 & 325 & .43 & 27,981 & 31,059 & 1.11 \\
\hline 2001 & 3,361 & 3,395 & 1.01 & 40,576 & 51,532 & 1.27 & 885 & 903 & 1.02 & 10,114 & 12,845 & 1.27 & 1,570 & 707 & .45 & 25,891 & 30,810 & 1.19 \\
\hline 2002 & 4,645 & 5,620 & 1.21 & 37,703 & 49,014 & 1.30 & 864 & 1,158 & 1.34 & 15,026 & 19,384 & 1.29 & 1,220 & 695 & .57 & 23,212 & 26,694 & 1.15 \\
\hline 2003 & 5,620 & 6,294 & 1.12 & 35,741 & 49,323 & 1.38 & 2,089 & 2,298 & 1.10 & 13,195 & 17,945 & 1.36 & 1,840 & 1,362 & .74 & 22,465 & 27,632 & 1.23 \\
\hline Average & 3,725 & 3,509 & .93 & 37,171 & 41,812 & 1.13 & 1,219 & 1,005 & .83 & 8,257 & 9,645 & 1.14 & 1,627 & 800 & .48 & 27,980 & 29,015 & 1.04 \\
\hline
\end{tabular}


Table 2. Irrigation water use, acres irrigated, and irrigation application rate by crop type for counties in the Groundwater Management Districts within the Kansas High Plains, 1991-2003-Continued

[Data from Kansas Water Office and Kansas Department of Agriculture (1993-2005). acre-ft, acre-foot; acre-ft/acre, acre-foot per acre]

\begin{tabular}{|c|c|c|c|c|c|c|c|c|c|c|c|c|c|c|c|c|c|c|}
\hline \multirow[b]{2}{*}{ Year } & \multicolumn{3}{|c|}{ Alfalfa } & \multicolumn{3}{|c|}{ Corn } & \multicolumn{3}{|c|}{ Grain sorghum } & \multicolumn{3}{|c|}{ Soybeans } & \multicolumn{3}{|c|}{ Wheat } & \multicolumn{3}{|c|}{ Other/Multiple use } \\
\hline & $\begin{array}{c}\text { Total } \\
\text { acres } \\
\text { irrigated }\end{array}$ & $\begin{array}{l}\text { Irriga- } \\
\text { tion } \\
\text { water } \\
\text { used } \\
\text { (acre-ft) }\end{array}$ & $\begin{array}{c}\text { Rate of } \\
\text { irri- } \\
\text { gation } \\
\text { (acre- } \\
\text { ft/acre) }\end{array}$ & $\begin{array}{c}\text { Total } \\
\text { acres } \\
\text { irrigated }\end{array}$ & $\begin{array}{c}\text { Irriga- } \\
\text { tion } \\
\text { water } \\
\text { used } \\
\text { (acre-ft) }\end{array}$ & $\begin{array}{l}\text { Rate of } \\
\text { irri- } \\
\text { gation } \\
\text { (acre- } \\
\text { ft/acre) }\end{array}$ & $\begin{array}{c}\text { Total } \\
\text { acres } \\
\text { irrigated }\end{array}$ & $\begin{array}{l}\text { Irriga- } \\
\text { tion } \\
\text { water } \\
\text { used } \\
\text { (acre-ft) }\end{array}$ & $\begin{array}{c}\text { Rate of } \\
\text { irri- } \\
\text { gation } \\
\text { (acre- } \\
\mathrm{ft} / \text { acre) }\end{array}$ & $\begin{array}{c}\text { Total } \\
\text { acres } \\
\text { irrigated }\end{array}$ & $\begin{array}{l}\text { Irriga- } \\
\text { tion } \\
\text { water } \\
\text { used } \\
\text { (acre-ft) }\end{array}$ & $\begin{array}{c}\text { Rate of } \\
\text { irri- } \\
\text { gation } \\
\text { (acre- } \\
\text { ft/acre) }\end{array}$ & $\begin{array}{c}\text { Total } \\
\text { acres } \\
\text { irrigated }\end{array}$ & $\begin{array}{l}\text { Irriga- } \\
\text { tion } \\
\text { water } \\
\text { used } \\
\text { (acre-ft) }\end{array}$ & $\begin{array}{l}\text { Rate of } \\
\text { irri- } \\
\text { gation } \\
\text { (acre- } \\
\text { ft/acre) }\end{array}$ & $\begin{array}{c}\text { Total } \\
\text { acres } \\
\text { irrigated }\end{array}$ & $\begin{array}{c}\text { Irriga- } \\
\text { tion } \\
\text { water } \\
\text { used } \\
\text { (acre-ft) }\end{array}$ & $\begin{array}{c}\text { Rate of } \\
\text { irri- } \\
\text { gation } \\
\text { (acre- } \\
\text { ft/acre) }\end{array}$ \\
\hline \multicolumn{19}{|c|}{ Stanton County } \\
\hline 1991 & 290 & 400 & 1.38 & 5,852 & 9,656 & 1.65 & 406 & 857 & 2.11 & 0 & 0 & 0 & 4,373 & 4,592 & 1.05 & 141,050 & 221,449 & 1.57 \\
\hline 1992 & 880 & 1,461 & 1.66 & 4,592 & 6,934 & 1.51 & 2,027 & 1,500 & .74 & 0 & 0 & 0 & 5,859 & 4,629 & .79 & 137,745 & 206,618 & 1.50 \\
\hline 1993 & 949 & 1,300 & 1.37 & 9,892 & 14,047 & 1.42 & 502 & 628 & 1.25 & 0 & 0 & 0 & 4,190 & 3,562 & .85 & 127,801 & 159,751 & 1.25 \\
\hline 1994 & 776 & 1,513 & 1.95 & 9,692 & 16,961 & 1.75 & 292 & 298 & 1.02 & 0 & 0 & 0 & 2,963 & 2,400 & .81 & 126,386 & 176,940 & 1.40 \\
\hline 1995 & 530 & 843 & 1.59 & 10,539 & 18,443 & 1.75 & 673 & 814 & 1.21 & 0 & 0 & 0 & 3,000 & 2,040 & .68 & 119,854 & 160,604 & 1.34 \\
\hline 1996 & 610 & 939 & 1.54 & 10,927 & 18,467 & 1.69 & 972 & 953 & .98 & 0 & 0 & 0 & 2,099 & 1,826 & .87 & 116,075 & 134,647 & 1.16 \\
\hline 1997 & 1,450 & 2,349 & 1.62 & 13,558 & 19,252 & 1.42 & 1,601 & 1,537 & .96 & 0 & 0 & 0 & 2,929 & 1,465 & .50 & 115,564 & 121,342 & 1.05 \\
\hline 1998 & 2,552 & 2,731 & 1.07 & 16,283 & 21,656 & 1.33 & 898 & 853 & .95 & 0 & 0 & 0 & 1,575 & 677 & .43 & 113,556 & 114,692 & 1.01 \\
\hline 1999 & 2,250 & 2,048 & .91 & 14,390 & 20,434 & 1.42 & 570 & 507 & .89 & 0 & 0 & 0 & 1,248 & 786 & .63 & 117,456 & 126,852 & 1.08 \\
\hline 2000 & 3,370 & 3,471 & 1.03 & 18,424 & 30,031 & 1.63 & 120 & 31 & .26 & 0 & 0 & 0 & 1,370 & 877 & .64 & 109,224 & 133,253 & 1.22 \\
\hline 2001 & 3,450 & 4,520 & 1.31 & 19,493 & 24,756 & 1.27 & 795 & 795 & 10 & 0 & 0 & 0 & 2,329 & 2,073 & .89 & 101,293 & 93,190 & .92 \\
\hline 2002 & 2,751 & 5,034 & 1.83 & 19,598 & 28,613 & 1.46 & 632 & 910 & 1.44 & 0 & 0 & 0 & 1,758 & 1,301 & .74 & 117,076 & 125,271 & 1.07 \\
\hline 2003 & 3,620 & 5,285 & 1.46 & 11,186 & 17,115 & 1.53 & 632 & 430 & .68 & 360 & 360 & 10 & 3,937 & 2,402 & .61 & 96,928 & 112,436 & 1.16 \\
\hline Average & 1,806 & 2,453 & 1.44 & 12,648 & 18,951 & 1.53 & 778 & 778 & 1.04 & 28 & 28 & .08 & 2,895 & 2,202 & .73 & 118,462 & 145,157 & 1.21 \\
\hline \multicolumn{19}{|c|}{ Stevens County } \\
\hline 1991 & 6,340 & 13,251 & 2.09 & 15,216 & 29,215 & 1.92 & 2,715 & 4,507 & 1.66 & 0 & 0 & 0 & 3,699 & 4,809 & 1.30 & 109,545 & 184,036 & 1.68 \\
\hline 1992 & 5,011 & 10,423 & 2.08 & 16,789 & 30,724 & 1.83 & 1,841 & 2,154 & 1.17 & 255 & 204 & .80 & 2,234 & 2,882 & 1.29 & 114,234 & 179,347 & 1.57 \\
\hline 1993 & 4,875 & 7,410 & 1.52 & 19,752 & 29,035 & 1.47 & 245 & 255 & 1.04 & 0 & 0 & 0 & 2,026 & 1,722 & .85 & 109,452 & 142,288 & 1.30 \\
\hline 1994 & 5,448 & 10,515 & 1.93 & 22,001 & 36,522 & 1.66 & 60 & 16 & .27 & 0 & 0 & 0 & 1,742 & 1,516 & .87 & 108,376 & 158,229 & 1.46 \\
\hline 1995 & 4,740 & 10,949 & 2.31 & 22,676 & 37,415 & 1.65 & 872 & 1,247 & 1.43 & 0 & 0 & 0 & 2,698 & 2,455 & .91 & 109,020 & 156,989 & 1.44 \\
\hline 1996 & 4,664 & 9,981 & 2.14 & 25,744 & 47,112 & 1.83 & 1,015 & 1,654 & 1.63 & 485 & 858 & 1.77 & 1,221 & 965 & .79 & 108,242 & 151,539 & 1.40 \\
\hline 1997 & 5,875 & 10,751 & 1.83 & 35,609 & 53,414 & 1.50 & 462 & 587 & 1.27 & 215 & 217 & 1.01 & 1,261 & 870 & .69 & 113,120 & 141,400 & 1.25 \\
\hline 1998 & 4,798 & 9,164 & 1.91 & 43,645 & 65,031 & 1.49 & 80 & 78 & .98 & 395 & 419 & 1.06 & 1,207 & 338 & .28 & 111,014 & 142,098 & 1.28 \\
\hline 1999 & 2,778 & 4,111 & 1.48 & 49,687 & 71,052 & 1.43 & 1,353 & 501 & .37 & 890 & 1,121 & 1.26 & 2,207 & 1,081 & .49 & 112,169 & 120,021 & 1.07 \\
\hline 2000 & 2,201 & 3,390 & 1.54 & 62,127 & 100,646 & 1.62 & 0 & 0 & 0 & 252 & 277 & 1.10 & 2,276 & 2,071 & .91 & 105,051 & 130,263 & 1.24 \\
\hline 2001 & 2,412 & 3,883 & 1.61 & 58,009 & 84,693 & 1.46 & 475 & 290 & .61 & 906 & 788 & .87 & 3,033 & 1,547 & .51 & 103,840 & 119,416 & 1.15 \\
\hline 2002 & 2,843 & 4,862 & 1.71 & 50,906 & 101,303 & 1.99 & 860 & 697 & .81 & 1,359 & 2,732 & 2.01 & 2,611 & 2,924 & 1.12 & 113,490 & 170,235 & 1.50 \\
\hline 2003 & 3,928 & 5,264 & 1.34 & 49,490 & 70,771 & 1.43 & 2,183 & 939 & .43 & 120 & 162 & 1.35 & 3,235 & 2,750 & .85 & 112,093 & 123,302 & 1.10 \\
\hline Average & 4,301 & 7,996 & 1.81 & 36,281 & 58,226 & 1.64 & 935 & 994 & .90 & 375 & 521 & .86 & 2,265 & 1,995 & .84 & 109,973 & 147,628 & 1.34 \\
\hline
\end{tabular}


Table 2. Irrigation water use, acres irrigated, and irrigation application rate by crop type for counties in the Groundwater Management Districts within the Kansas High Plains, 1991-2003-Continued

[Data from Kansas Water Office and Kansas Department of Agriculture (1993-2005). acre-ft, acre-foot; acre-ft/acre, acre-foot per acre]

\begin{tabular}{|c|c|c|c|c|c|c|c|c|c|c|c|c|c|c|c|c|c|c|}
\hline \multirow[b]{2}{*}{ Year } & \multicolumn{3}{|c|}{ Alfalfa } & \multicolumn{3}{|c|}{ Corn } & \multicolumn{3}{|c|}{ Grain sorghum } & \multicolumn{3}{|c|}{ Soybeans } & \multicolumn{3}{|c|}{ Wheat } & \multicolumn{3}{|c|}{ Other/Multiple use } \\
\hline & $\begin{array}{c}\text { Total } \\
\text { acres } \\
\text { irrigated }\end{array}$ & $\begin{array}{l}\text { Irriga- } \\
\text { tion } \\
\text { water } \\
\text { used } \\
\text { (acre-ft) }\end{array}$ & $\begin{array}{l}\text { Rate of } \\
\text { irri- } \\
\text { gation } \\
\text { (acre- } \\
\text { ft/acre) }\end{array}$ & $\begin{array}{c}\text { Total } \\
\text { acres } \\
\text { irrigated }\end{array}$ & $\begin{array}{l}\text { Irriga- } \\
\text { tion } \\
\text { water } \\
\text { used } \\
\text { (acre-ft) }\end{array}$ & $\begin{array}{l}\text { Rate of } \\
\text { irri- } \\
\text { gation } \\
\text { (acre- } \\
\mathrm{ft} / \text { acre) }\end{array}$ & $\begin{array}{c}\text { Total } \\
\text { acres } \\
\text { irrigated }\end{array}$ & $\begin{array}{l}\text { Irriga- } \\
\text { tion } \\
\text { water } \\
\text { used } \\
\text { (acre-ft) }\end{array}$ & $\begin{array}{l}\text { Rate of } \\
\text { irri- } \\
\text { gation } \\
\text { (acre- } \\
\text { ft/acre) }\end{array}$ & $\begin{array}{c}\text { Total } \\
\text { acres } \\
\text { irrigated }\end{array}$ & $\begin{array}{l}\text { Irriga- } \\
\text { tion } \\
\text { water } \\
\text { used } \\
\text { (acre-ft) }\end{array}$ & $\begin{array}{l}\text { Rate of } \\
\text { irri- } \\
\text { gation } \\
\text { (acre- } \\
\text { ft/acre) }\end{array}$ & $\begin{array}{c}\text { Total } \\
\text { acres } \\
\text { irrigated }\end{array}$ & $\begin{array}{l}\text { Irriga- } \\
\text { tion } \\
\text { water } \\
\text { used } \\
\text { (acre-ft) }\end{array}$ & $\begin{array}{l}\text { Rate of } \\
\text { irri- } \\
\text { gation } \\
\text { (acre- } \\
\text { ft/acre) }\end{array}$ & $\begin{array}{c}\text { Total } \\
\text { acres } \\
\text { irrigated }\end{array}$ & $\begin{array}{l}\text { Irriga- } \\
\text { tion } \\
\text { water } \\
\text { used } \\
\text { (acre-ft) }\end{array}$ & $\begin{array}{l}\text { Rate of } \\
\text { irri- } \\
\text { gation } \\
\text { (acre- } \\
\text { ft/acre) }\end{array}$ \\
\hline \multicolumn{19}{|c|}{ Thomas County } \\
\hline 1991 & 533 & 965 & 1.81 & 54,600 & 82,992 & 1.52 & 5,373 & 6,394 & 1.19 & 2,542 & 2,872 & 1.13 & 3,217 & 1,512 & 0.47 & 31,362 & 37,321 & 1.19 \\
\hline 1992 & 901 & 649 & .72 & 44,989 & 32,842 & .73 & 11,795 & 7,785 & .66 & 3,345 & 2,174 & .65 & 3,417 & 1,674 & .49 & 28,353 & 17,862 & .63 \\
\hline 1993 & 561 & 847 & 1.51 & 57,875 & 49,773 & .86 & 2,178 & 1,024 & .47 & 948 & 502 & .53 & 2,198 & 835 & .38 & 31,403 & 18,528 & .59 \\
\hline 1994 & 826 & 1,330 & 1.61 & 60,345 & 73,621 & 1.22 & 2,021 & 1,597 & .79 & 2,108 & 2,045 & .97 & 1,422 & 569 & .40 & 32,015 & 28,173 & .88 \\
\hline 1995 & 734 & 749 & 1.02 & 56,035 & 67,242 & 1.20 & 1,605 & 1,316 & .82 & 1,651 & 1,833 & 1.11 & 3,304 & 958 & .29 & 31,998 & 26,878 & .84 \\
\hline 1996 & 815 & 791 & .97 & 67,052 & 72,416 & 1.08 & 1,427 & 1,127 & .79 & 1,701 & 1,412 & .83 & 1,987 & 934 & .47 & 25,871 & 21,732 & .84 \\
\hline 1997 & 900 & 1,233 & 1.37 & 72,028 & 83,552 & 1.16 & 1,146 & 1,020 & .89 & 2,354 & 2,613 & 1.11 & 2,229 & 1,271 & .57 & 21,318 & 20,252 & .95 \\
\hline 1998 & 1,213 & 1,589 & 1.31 & 65,349 & 71,230 & 1.09 & 1,008 & 615 & .61 & 4,004 & 3,604 & .90 & 1,835 & 1,119 & .61 & 25,186 & 20,653 & .82 \\
\hline 1999 & 747 & 769 & 1.03 & 63,680 & 57,949 & .91 & 420 & 265 & .63 & 4,080 & 2,897 & .71 & 2,175 & 1,088 & .50 & 28,088 & 19,662 & .70 \\
\hline 2000 & 722 & 989 & 1.37 & 63,197 & 89,740 & 1.42 & 242 & 174 & .72 & 5,303 & 6,311 & 1.19 & 1,939 & 989 & .51 & 30,291 & 35,743 & 1.18 \\
\hline 2001 & 1,161 & 1,416 & 1.22 & 54,418 & 68,023 & 1.25 & 1,463 & 775 & .53 & 6,318 & 6,823 & 1.08 & 3,369 & 1,516 & .45 & 33,135 & 31,478 & .95 \\
\hline 2002 & 1,508 & 2,533 & 1.68 & 52,141 & 76,126 & 1.46 & 1,032 & 815 & .79 & 6,084 & 8,153 & 1.34 & 1,793 & 1,488 & .83 & 38,968 & 46,372 & 1.19 \\
\hline 2003 & 1,673 & 2,443 & 1.46 & 38,859 & 54,014 & 1.39 & 2,647 & 2,118 & .80 & 6,458 & 7,039 & 1.09 & 5,595 & 3,917 & .70 & 46,320 & 51,878 & 1.12 \\
\hline Average & 946 & 1,254 & 1.31 & 57,736 & 67,655 & 1.18 & 2,489 & 1,925 & .75 & 3,607 & 3,714 & .97 & 2,652 & 1,375 & .51 & 31,101 & 28,964 & .91 \\
\hline \multicolumn{19}{|c|}{ Wallace County } \\
\hline 1991 & 1,248 & 2,309 & 1.85 & 16,422 & 25,454 & 1.55 & 1,481 & 1,377 & .93 & 40 & 113 & 2.82 & 3,454 & 1,692 & .49 & 37,716 & 56,197 & 1.49 \\
\hline 1992 & 1,271 & 2,072 & 1.63 & 19,840 & 26,387 & 1.33 & 1,771 & 1,913 & 1.08 & 120 & 126 & 1.05 & 3,000 & 1,650 & .55 & 30,741 & 35,660 & 1.16 \\
\hline 1993 & 1,347 & 1,576 & 1.17 & 20,016 & 21,617 & 1.08 & 1,045 & 700 & .67 & 0 & 0 & 0 & 2,430 & 729 & .30 & 33,437 & 29,759 & .89 \\
\hline 1994 & 1,889 & 2,588 & 1.37 & 20,420 & 31,038 & 1.52 & 669 & 622 & .93 & 0 & 0 & 0 & 1,691 & 609 & .36 & 34,339 & 40,520 & 1.18 \\
\hline 1995 & 1,473 & 2,121 & 1.44 & 18,761 & 26,265 & 1.40 & 856 & 805 & .94 & 0 & 0 & 0 & 3,698 & 2,145 & .58 & 32,445 & 37,636 & 1.16 \\
\hline 1996 & 1,803 & 3,137 & 1.74 & 24,211 & 35,590 & 1.47 & 341 & 280 & .82 & 0 & 0 & 0 & 2,727 & 1,445 & .53 & 32,513 & 39,991 & 1.23 \\
\hline 1997 & 1,481 & 2,310 & 1.56 & 25,094 & 31,117 & 1.24 & 430 & 232 & .54 & 0 & 0 & 0 & 5,621 & 3,035 & .54 & 27,947 & 27,668 & .99 \\
\hline 1998 & 1,018 & 1,619 & 1.59 & 24,538 & 31,899 & 1.30 & 640 & 282 & .44 & 245 & 196 & .80 & 4,416 & 3,047 & .69 & 29,716 & 29,419 & .99 \\
\hline 1999 & 913 & 1,187 & 1.30 & 23,337 & 27,071 & 1.16 & 346 & 197 & .57 & 495 & 396 & .80 & 2,359 & 1,203 & .51 & 33,210 & 29,557 & .89 \\
\hline 2000 & 1,007 & 1,541 & 1.53 & 24,094 & 37,828 & 1.57 & 369 & 284 & .77 & 130 & 215 & 1.65 & 2,131 & 1,641 & .77 & 33,101 & 40,052 & 1.21 \\
\hline 2001 & 1,314 & 2,707 & 2.06 & 19,374 & 27,705 & 1.43 & 665 & 479 & .72 & 250 & 363 & 1.45 & 2,437 & 1,365 & .56 & 35,958 & 37,396 & 1.04 \\
\hline 2002 & 1,211 & 1,720 & 1.42 & 19,482 & 32,535 & 1.67 & 253 & 235 & .93 & 365 & 299 & .82 & 2,596 & 1,713 & .66 & 35,320 & 48,388 & 1.37 \\
\hline 2003 & 1,409 & 1,930 & 1.37 & 13,876 & 19,426 & 1.40 & 257 & 129 & .50 & 247 & 319 & 1.29 & 4,572 & 2,789 & .61 & 36,522 & 40,539 & 1.11 \\
\hline Average & 1,337 & 2,063 & 1.54 & 20,728 & 28,764 & 1.39 & 702 & 580 & .76 & 146 & 156 & .82 & 3,164 & 1,774 & .55 & 33,305 & 37,906 & 1.13 \\
\hline
\end{tabular}


Table 2. Irrigation water use, acres irrigated, and irrigation application rate by crop type for counties in the Groundwater Management Districts within the Kansas High Plains, 1991-2003-Continued

[Data from Kansas Water Office and Kansas Department of Agriculture (1993-2005). acre-ft, acre-foot; acre-ft/acre, acre-foot per acre]

\begin{tabular}{|c|c|c|c|c|c|c|c|c|c|c|c|c|c|c|c|c|c|c|}
\hline \multirow[b]{2}{*}{ Year } & \multicolumn{3}{|c|}{ Alfalfa } & \multicolumn{3}{|c|}{ Corn } & \multicolumn{3}{|c|}{ Grain sorghum } & \multicolumn{3}{|c|}{ Soybeans } & \multicolumn{3}{|c|}{ Wheat } & \multicolumn{3}{|c|}{ Other/Multiple use } \\
\hline & $\begin{array}{c}\text { Total } \\
\text { acres } \\
\text { irrigated }\end{array}$ & $\begin{array}{c}\text { Irriga- } \\
\text { tion } \\
\text { water } \\
\text { used } \\
\text { (acre-ft) }\end{array}$ & $\begin{array}{c}\text { Rate of } \\
\text { irri- } \\
\text { gation } \\
\text { (acre- } \\
\text { ft/acre) }\end{array}$ & $\begin{array}{c}\text { Total } \\
\text { acres } \\
\text { irrigated }\end{array}$ & $\begin{array}{c}\text { Irriga- } \\
\text { tion } \\
\text { water } \\
\text { used } \\
\text { (acre-ft) }\end{array}$ & $\begin{array}{l}\text { Rate of } \\
\text { irri- } \\
\text { gation } \\
\text { (acre- } \\
\text { ft/acre) }\end{array}$ & $\begin{array}{c}\text { Total } \\
\text { acres } \\
\text { irrigated }\end{array}$ & $\begin{array}{c}\text { Irriga- } \\
\text { tion } \\
\text { water } \\
\text { used } \\
\text { (acre-ft) }\end{array}$ & $\begin{array}{l}\text { Rate of } \\
\text { irri- } \\
\text { gation } \\
\text { (acre- } \\
\text { ft/acre) }\end{array}$ & $\begin{array}{c}\text { Total } \\
\text { acres } \\
\text { irrigated }\end{array}$ & $\begin{array}{c}\text { Irriga- } \\
\text { tion } \\
\text { water } \\
\text { used } \\
\text { (acre-ft) }\end{array}$ & $\begin{array}{c}\text { Rate of } \\
\text { irri- } \\
\text { gation } \\
\text { (acre- } \\
\text { ft/acre) }\end{array}$ & $\begin{array}{c}\text { Total } \\
\text { acres } \\
\text { irrigated }\end{array}$ & $\begin{array}{c}\text { Irriga- } \\
\text { tion } \\
\text { water } \\
\text { used } \\
\text { (acre-ft) }\end{array}$ & $\begin{array}{l}\text { Rate of } \\
\text { irri- } \\
\text { gation } \\
\text { (acre- } \\
\text { ft/acre) }\end{array}$ & $\begin{array}{c}\text { Total } \\
\text { acres } \\
\text { irrigated }\end{array}$ & $\begin{array}{c}\text { Irriga- } \\
\text { tion } \\
\text { water } \\
\text { used } \\
\text { (acre-ft) }\end{array}$ & $\begin{array}{l}\text { Rate of } \\
\text { irri- } \\
\text { gation } \\
\text { (acre- } \\
\text { ft/acre) }\end{array}$ \\
\hline \multicolumn{19}{|c|}{ Wichita County } \\
\hline 1991 & 403 & 669 & 1.66 & 3,936 & 6,691 & 1.70 & 3,915 & 4,933 & 1.26 & 261 & 318 & 1.22 & 2,383 & 1,978 & 0.83 & 79,313 & 104,693 & 1.32 \\
\hline 1992 & 420 & 735 & 1.75 & 2,660 & 2,899 & 1.09 & 2,568 & 2,208 & .86 & 206 & 130 & .63 & 1,896 & 1,536 & .81 & 76,929 & 82,314 & 1.07 \\
\hline 1993 & 396 & 372 & .94 & 9,792 & 10,380 & 1.06 & 4,102 & 3,733 & .91 & 0 & 0 & 0 & 2,197 & 945 & .43 & 57,668 & 46,711 & .81 \\
\hline 1994 & 570 & 781 & 1.37 & 4,993 & 7,340 & 1.47 & 4,106 & 3,819 & .93 & 120 & 60 & .50 & 1,489 & 983 & .66 & 68,753 & 77,003 & 1.12 \\
\hline 1995 & 342 & 595 & 1.74 & 7,911 & 10,601 & 1.34 & 3,800 & 3,382 & .89 & 0 & 0 & 0 & 1,744 & 1,325 & .76 & 66,962 & 65,623 & .98 \\
\hline 1996 & 674 & 674 & 10 & 10,975 & 12,951 & 1.18 & 4,211 & 3,706 & .88 & 118 & 81 & .69 & 738 & 391 & .53 & 63,061 & 60,539 & .96 \\
\hline 1997 & 861 & 1,076 & 1.25 & 11,011 & 11,782 & 1.07 & 3,376 & 2,296 & .68 & 366 & 264 & .72 & 1,914 & 1,225 & .64 & 64,660 & 54,961 & .85 \\
\hline 1998 & 947 & 1,127 & 1.19 & 11,121 & 13,012 & 1.17 & 1,015 & 619 & .61 & 282 & 133 & .47 & 824 & 577 & .70 & 63,307 & 52,545 & .83 \\
\hline 1999 & 712 & 790 & 1.11 & 11,617 & 13,824 & 1.19 & 403 & 520 & 1.29 & 515 & 633 & 1.23 & 2,410 & 1,277 & .53 & 66,561 & 53,249 & .80 \\
\hline 2000 & 666 & 852 & 1.28 & 10,155 & 14,826 & 1.46 & 773 & 665 & .86 & 541 & 487 & .90 & 2,683 & 1,583 & .59 & 68,529 & 67,844 & .99 \\
\hline 2001 & 434 & 573 & 1.32 & 10,307 & 10,513 & 1.02 & 1,420 & 966 & .68 & 357 & 332 & .93 & 1,419 & 568 & .40 & 61,413 & 49,130 & .80 \\
\hline 2002 & 327 & 592 & 1.81 & 7,682 & 11,600 & 1.51 & 1,371 & 1,549 & 1.13 & 471 & 542 & 1.15 & 1,182 & 898 & .76 & 70,464 & 71,169 & 1.01 \\
\hline 2003 & 347 & 507 & 1.46 & 5,321 & 6,811 & 1.28 & 1,509 & 936 & .62 & 106 & 123 & 1.16 & 3,386 & 1,727 & .51 & 65,012 & 53,960 & .83 \\
\hline Average & 546 & 719 & 1.38 & 8,268 & 10,248 & 1.27 & 2,505 & 2,256 & .89 & 257 & 239 & .74 & 1,867 & 1,155 & .63 & 67,126 & 64,595 & .95 \\
\hline
\end{tabular}


Table 3. Irrigation water use, acres irrigated, and irrigation application rate by system type for the Groundwater Management Districts within the Kansas High Plains, 1991-2003.

[Data from Kansas Water Office and Kansas Department of Agriculture (1993-2005). acre-ft/acre, acre-foot per acre]

\begin{tabular}{|c|c|c|c|c|c|c|c|c|c|c|c|c|c|c|c|c|c|}
\hline \multirow{2}{*}{$\begin{array}{c}\text { Ground- } \\
\text { water } \\
\text { Manage- } \\
\text { ment } \\
\text { District } \\
\text { (GMD) } \\
\text { number } \\
\text { (fig. 1) }\end{array}$} & \multicolumn{2}{|c|}{$\begin{array}{c}\text { Total irrigation water } \\
\text { use }\end{array}$} & \multicolumn{2}{|c|}{ Total acres irrigated } & \multirow{2}{*}{$\begin{array}{l}\text { Aver- } \\
\text { age } \\
\text { rate of } \\
\text { irri- } \\
\text { gation } \\
\text { (acre- } \\
\mathrm{ft} / \mathrm{yr})\end{array}$} & \multicolumn{3}{|c|}{ Flood irrigation } & \multicolumn{3}{|c|}{ Center pivot irrigation } & \multicolumn{3}{|c|}{$\begin{array}{l}\text { Center pivot irrigation with drop } \\
\text { nozzle }\end{array}$} & \multicolumn{3}{|c|}{ Other irrigation systems } \\
\hline & Acre-feet & $\begin{array}{l}\text { Percen- } \\
\text { tage of } \\
\text { total for } \\
\text { all GMDs }\end{array}$ & Acres & $\begin{array}{l}\text { Percen- } \\
\text { tage of } \\
\text { total for } \\
\text { all GMDs }\end{array}$ & & $\begin{array}{l}\text { Total acres } \\
\text { irrigated }\end{array}$ & $\begin{array}{l}\text { Water used } \\
\text { (acre-ft) }\end{array}$ & $\begin{array}{l}\text { Rate of } \\
\text { irri- } \\
\text { gation } \\
\text { (acre- } \\
\text { ft/acre) }\end{array}$ & $\begin{array}{l}\text { Total acres } \\
\text { irrigated }\end{array}$ & $\begin{array}{l}\text { Water used } \\
\text { (acre-ft) }\end{array}$ & $\begin{array}{l}\text { Rate of } \\
\text { irri- } \\
\text { gation } \\
\text { (acre- } \\
\text { ft/acre) }\end{array}$ & $\begin{array}{l}\text { Total acres } \\
\text { irrigated }\end{array}$ & $\begin{array}{l}\text { Water used } \\
\text { (acre-ft) }\end{array}$ & $\begin{array}{l}\text { Rate of } \\
\text { irri- } \\
\text { gation } \\
\text { (acre- } \\
\text { ft/acre) }\end{array}$ & $\begin{array}{l}\text { Total acres } \\
\text { irrigated }\end{array}$ & $\begin{array}{l}\text { Water used } \\
\text { (acre-ft) }\end{array}$ & $\begin{array}{c}\text { Rate of } \\
\text { irri- } \\
\text { gation } \\
\text { (acre- } \\
\text { ft/acre) }\end{array}$ \\
\hline \multicolumn{18}{|c|}{1991} \\
\hline 1 & 355,589 & 8.4 & 262,528 & 9.6 & 1.35 & 216,196 & 299,120 & 1.38 & 34,172 & 39,671 & 1.16 & 0 & 0 & 0 & 12,160 & 16,798 & 1.38 \\
\hline 2 & 112,604 & 2.7 & 97,833 & 3.6 & 1.15 & 37,604 & 47,438 & 1.26 & 52,475 & 57,681 & 1.10 & 0 & 0 & 0 & 7,754 & 7,485 & .97 \\
\hline 3 & $2,660,233$ & 63.1 & $1,593,191$ & 58.0 & 1.67 & 823,757 & $1,417,562$ & 1.72 & 604,248 & 974,993 & 1.61 & 0 & 0 & 0 & 165,186 & 267,678 & 1.62 \\
\hline 4 & 478,854 & 11.4 & 360,109 & 13.1 & 1.33 & 111,066 & 173,659 & 1.56 & 218,417 & 263,077 & 1.20 & 0 & 0 & 0 & 30,626 & 42,118 & 1.38 \\
\hline 5 & 606,672 & 14.4 & 434,745 & 15.8 & 1.40 & 66,906 & 98,928 & 1.48 & 358,046 & 494,995 & 1.38 & 0 & 0 & 0 & 9,793 & 12,749 & 1.30 \\
\hline Totals & $4,213,952$ & 100 & $2,748,406$ & 100 & 1.53 & $1,255,529$ & $2,036,707$ & 1.62 & $1,267,358$ & $1,830,417$ & 1.44 & 0 & 0 & 0 & 225,519 & 346,828 & 1.54 \\
\hline \multicolumn{18}{|c|}{1992} \\
\hline 1 & 270,143 & 8.7 & 244,582 & 9.1 & 1.10 & 188,730 & 216,103 & 1.15 & 30,403 & 30,008 & .99 & 12,209 & 13,003 & 1.07 & 13,240 & 11,029 & .83 \\
\hline 2 & 57,195 & 1.8 & 92,880 & 3.5 & .62 & 33,125 & 22,523 & .68 & 52,029 & 30,556 & .59 & 1,608 & 619 & .38 & 6,118 & 3,497 & .57 \\
\hline 3 & $2,178,273$ & 70.2 & $1,580,084$ & 59.0 & 1.38 & 783,385 & $1,120,560$ & 1.43 & 574,112 & 773,720 & 1.35 & 52,305 & 62,683 & 1.20 & 170,282 & 221,310 & 1.30 \\
\hline 4 & 268,217 & 8.6 & 340,522 & 12.7 & .79 & 83,917 & 82,776 & .99 & 186,421 & 133,135 & .71 & 43,379 & 31,187 & .72 & 26,805 & 21,119 & .79 \\
\hline 5 & 330,406 & 10.6 & 418,749 & 15.6 & .79 & 53,548 & 34,700 & .65 & 342,227 & 278,906 & .81 & 15,919 & 11,997 & .75 & 7,055 & 4,803 & .68 \\
\hline Totals & $3,104,234$ & 100 & $2,676,817$ & 100 & 1.16 & $1,142,705$ & $1,476,662$ & 1.29 & $1,185,192$ & $1,246,325$ & 1.05 & 125,420 & 119,489 & .95 & 223,500 & 261,758 & 1.17 \\
\hline \multicolumn{18}{|c|}{1993} \\
\hline 1 & 197,600 & 7.3 & 223,046 & 8.5 & .89 & 153,685 & 141,603 & .92 & 30,312 & 24,862 & .82 & 21,564 & 17,978 & .83 & 17,485 & 13,157 & .75 \\
\hline 2 & 59,150 & 2.2 & 90,525 & 3.5 & .65 & 31,261 & 22,283 & .71 & 52,474 & 33,068 & .63 & 1,048 & 711 & .68 & 5,742 & 3,088 & .54 \\
\hline 3 & $1,887,823$ & 69.6 & $1,550,396$ & 59.2 & 1.22 & 688,521 & 859,444 & 1.25 & 601,448 & 740,184 & 1.23 & 56,285 & 62,973 & 1.12 & 204,142 & 225,222 & 1.10 \\
\hline 4 & 261,648 & 9.6 & 343,660 & 13.1 & .76 & 77,960 & 79,034 & 1.01 & 180,509 & 127,103 & .70 & 60,882 & 38,516 & .63 & 24,309 & 16,995 & .70 \\
\hline 5 & 308,474 & 11.4 & 410,330 & 15.7 & .75 & 48,087 & 27,207 & .57 & 346,260 & 268,263 & .77 & 8,495 & 8,095 & .95 & 7,488 & 4,909 & .66 \\
\hline Totals & $2,714,695$ & 100 & $2,617,957$ & 100 & 1.04 & 999,514 & $1,129,571$ & 1.13 & $1,211,003$ & $1,193,480$ & .99 & 148,274 & 128,273 & .87 & 259,166 & 263,371 & 1.02 \\
\hline \multicolumn{18}{|c|}{1994} \\
\hline 1 & 281,921 & 7.9 & 236,993 & 8.8 & 1.19 & 152,462 & 183,734 & 1.21 & 35,593 & 41,356 & 1.16 & 24,495 & 26,488 & 1.08 & 24,443 & 30,343 & 1.24 \\
\hline 2 & 100,583 & 2.8 & 97,243 & 3.6 & 1.03 & 33,648 & 38,253 & 1.14 & 53,525 & 52,899 & .99 & 1,108 & 872 & .79 & 8,962 & 8,559 & .96 \\
\hline 3 & $2,239,152$ & 62.5 & $1,555,565$ & 58.0 & 1.44 & 602,004 & 903,099 & 1.50 & 609,548 & 868,895 & 1.43 & 88,960 & 124,034 & 1.39 & 255,053 & 343,124 & 1.35 \\
\hline 4 & 396,603 & 11.1 & 367,070 & 13.7 & 1.08 & 74,031 & 97,211 & 1.31 & 184,188 & 191,807 & 1.04 & 73,774 & 73,634 & 1.00 & 35,077 & 33,951 & .97 \\
\hline 5 & 565,785 & 15.8 & 428,022 & 15.9 & 1.32 & 56,293 & 73,273 & 1.30 & 352,781 & 469,318 & 1.33 & 9,305 & 12,269 & 1.32 & 9,643 & 10,925 & 1.13 \\
\hline Totals & $3,584,044$ & 100 & $2,684,893$ & 100 & 1.33 & 918,438 & $1,295,570$ & 1.41 & $1,235,635$ & $1,624,275$ & 1.31 & 197,642 & 237,297 & 1.20 & 333,178 & 426,902 & 1.28 \\
\hline \multicolumn{18}{|c|}{1995} \\
\hline 1 & 258,857 & 7.9 & 242,512 & 9.1 & 1.07 & 136,550 & 155,854 & 1.14 & 41,565 & 40,900 & .98 & 33,328 & 32,913 & .99 & 31,069 & 29,190 & .94 \\
\hline 2 & 84,940 & 2.6 & 97,814 & 3.7 & .87 & 31,246 & 29,015 & .93 & 56,895 & 48,279 & .85 & 1,669 & 1,094 & .66 & 8,004 & 6,552 & .82 \\
\hline 3 & $2,109,913$ & 64.5 & $1,533,409$ & 57.8 & 1.38 & 486,039 & 708,401 & 1.46 & 657,248 & 882,965 & 1.34 & 93,271 & 125,052 & 1.34 & 296,851 & 393,495 & 1.33 \\
\hline 4 & 361,778 & 11.1 & 355,259 & 13.4 & 1.02 & 60,157 & 73,850 & 1.23 & 195,033 & 193,008 & .99 & 74,158 & 69,698 & .94 & 25,911 & 25,222 & .97 \\
\hline 5 & 453,515 & 13.9 & 426,810 & 16.1 & 1.06 & 57,069 & 56,766 & .99 & 348,025 & 375,065 & 1.08 & 14,367 & 14,473 & 1.01 & 7,349 & 7,211 & .98 \\
\hline Totals & $3,269,003$ & 100 & $2,655,804$ & 100 & 1.23 & 771,061 & $1,023,886$ & 1.33 & $1,298,766$ & $1,540,217$ & 1.19 & 216,793 & 243,230 & 1.12 & 369,184 & 461,670 & 1.25 \\
\hline
\end{tabular}


Table 3. Irrigation water use, acres irrigated, and irrigation application rate by system type for the Groundwater Management Districts within the Kansas High Plains, 1991-2003.-Continued

[Data from Kansas Water Office and Kansas Department of Agriculture (1993-2005). acre-ft/acre, acre-foot per acre]

\begin{tabular}{|c|c|c|c|c|c|c|c|c|c|c|c|c|c|c|c|c|c|}
\hline \multirow{2}{*}{$\begin{array}{c}\text { Ground- } \\
\text { water } \\
\text { Manage- } \\
\text { ment } \\
\text { District } \\
\text { (GMD) } \\
\text { number } \\
\text { (fig. 1) }\end{array}$} & \multicolumn{2}{|c|}{$\begin{array}{l}\text { Total irrigation water } \\
\text { use } \\
\end{array}$} & \multicolumn{2}{|c|}{ Total acres irrigated } & \multirow{2}{*}{$\begin{array}{l}\text { Aver- } \\
\text { age } \\
\text { rate of } \\
\text { irri- } \\
\text { gation } \\
\text { (acre- } \\
\mathrm{ft} / \mathrm{yr})\end{array}$} & \multicolumn{3}{|c|}{ Flood irrigation } & \multicolumn{3}{|c|}{ Center pivot irrigation } & \multicolumn{3}{|c|}{$\begin{array}{l}\text { Center pivot irrigation with drop } \\
\text { nozzle }\end{array}$} & \multicolumn{3}{|c|}{ Other irrigation systems } \\
\hline & Acre-feet & $\begin{array}{l}\text { Percen- } \\
\text { tage of } \\
\text { total for } \\
\text { all GMDs }\end{array}$ & Acres & $\begin{array}{l}\text { Percen- } \\
\text { tage of } \\
\text { total for } \\
\text { all GMDs }\end{array}$ & & $\begin{array}{c}\text { Total acres } \\
\text { irrigated }\end{array}$ & $\begin{array}{l}\text { Water used } \\
\text { (acre-ft) }\end{array}$ & $\begin{array}{l}\text { Rate of } \\
\text { irri- } \\
\text { gation } \\
\text { (acre- } \\
\text { ft/acre) }\end{array}$ & $\begin{array}{l}\text { Total acres } \\
\text { irrigated }\end{array}$ & $\begin{array}{l}\text { Water used } \\
\text { (acre-ft) }\end{array}$ & $\begin{array}{l}\text { Rate of } \\
\text { irri- } \\
\text { gation } \\
\text { (acre- } \\
\text { ft/acre) }\end{array}$ & $\begin{array}{l}\text { Total acres } \\
\text { irrigated }\end{array}$ & $\begin{array}{l}\text { Water used } \\
\text { (acre-ft) }\end{array}$ & $\begin{array}{l}\text { Rate of } \\
\text { irri- } \\
\text { gation } \\
\text { (acre- } \\
\text { ft/acre) }\end{array}$ & $\begin{array}{l}\text { Total acres } \\
\text { irrigated }\end{array}$ & $\begin{array}{l}\text { Water used } \\
\text { (acre-ft) }\end{array}$ & $\begin{array}{l}\text { Rate of } \\
\text { irri- } \\
\text { gation } \\
\text { (acre- } \\
\mathrm{ft} / \text { acre) }\end{array}$ \\
\hline \multicolumn{18}{|c|}{1996} \\
\hline 1 & 250,042 & 8.3 & 242,642 & 9.1 & 1.03 & 123,648 & 131,803 & 1.07 & 48,318 & 46,961 & 0.97 & 42,166 & 41,796 & 0.99 & 28,510 & 29,482 & 1.03 \\
\hline 2 & 86,645 & 2.9 & 103,597 & 3.9 & .84 & 29,702 & 28,537 & .96 & 63,406 & 50,110 & .79 & 2,289 & 1,924 & .84 & 8,200 & 6,074 & .74 \\
\hline 3 & $1,949,992$ & 64.5 & $1,524,257$ & 57.0 & 1.28 & 442,764 & 597,295 & 1.35 & 684,371 & 857,864 & 1.25 & 113,215 & 141,655 & 1.25 & 283,907 & 353,178 & 1.24 \\
\hline 4 & 364,688 & 12.1 & 370,641 & 13.9 & .98 & 52,973 & 62,599 & 1.18 & 199,944 & 192,755 & .96 & 85,604 & 78,055 & .91 & 32,120 & 31,279 & .97 \\
\hline 5 & 371,749 & 12.3 & 431,379 & 16.1 & .86 & 49,876 & 41,465 & .83 & 355,894 & 309,488 & .87 & 18,249 & 15,647 & .86 & 7,360 & 5,149 & .70 \\
\hline Totals & $3,023,116$ & 100 & $2,672,516$ & 100 & 1.13 & 698,963 & 861,699 & 1.23 & $1,351,933$ & $1,457,178$ & 1.08 & 261,523 & 279,077 & 1.07 & 360,097 & 425,162 & 1.18 \\
\hline \multicolumn{18}{|c|}{1997} \\
\hline 1 & 225,872 & 7.8 & 246,497 & 9.0 & .92 & 106,858 & 105,247 & .98 & 41,150 & 33,529 & .81 & 69,341 & 60,145 & .87 & 29,148 & 26,951 & .92 \\
\hline 2 & 76,457 & 2.6 & 106,771 & 3.9 & .72 & 27,709 & 22,793 & .82 & 42,920 & 29,687 & .69 & 28,158 & 18,578 & .66 & 7,984 & 5,399 & .68 \\
\hline 3 & $1,851,794$ & 63.7 & $1,576,430$ & 57.3 & 1.17 & 355,973 & 431,904 & 1.21 & 516,487 & 623,105 & 1.21 & 393,274 & 465,788 & 1.18 & 310,696 & 330,997 & 1.07 \\
\hline 4 & 413,066 & 14.2 & 381,935 & 13.9 & 1.08 & 44,908 & 54,950 & 1.22 & 136,971 & 142,795 & 1.04 & 170,738 & 184,679 & 1.08 & 29,318 & 30,642 & 1.05 \\
\hline 5 & 341,855 & 11.8 & 439,038 & 16.0 & .78 & 46,642 & 34,089 & .73 & 269,480 & 211,070 & .78 & 115,490 & 91,938 & .80 & 7,426 & 4,758 & .64 \\
\hline Totals & $2,909,044$ & 100 & $2,750,671$ & 100 & 1.06 & 582,090 & 648,983 & 1.11 & $1,007,008$ & $1,040,186$ & 1.03 & 777,001 & 821,128 & 1.06 & 384,572 & 398,747 & 1.04 \\
\hline \multicolumn{18}{|c|}{1998} \\
\hline 1 & 223,389 & 7.1 & 244,883 & 8.8 & .91 & 86,245 & 81,677 & .95 & 44,591 & 37,746 & .85 & 79,805 & 75,122 & .94 & 34,242 & 28,844 & .84 \\
\hline 2 & 100,825 & 3.2 & 110,441 & 4.0 & .91 & 26,502 & 27,643 & 1.04 & 39,608 & 32,975 & .83 & 32,294 & 29,975 & .93 & 12,037 & 10,232 & .85 \\
\hline 3 & $1,940,161$ & 61.6 & $1,583,403$ & 57.2 & 1.23 & 280,187 & 326,765 & 1.17 & 481,008 & 619,532 & 1.29 & 474,580 & 599,184 & 1.26 & 347,628 & 394,680 & 1.14 \\
\hline 4 & 376,852 & 12.0 & 380,712 & 13.8 & .99 & 32,801 & 36,220 & 1.10 & 104,022 & 101,738 & .98 & 212,541 & 209,301 & .98 & 31,348 & 29,593 & .94 \\
\hline 5 & 511,214 & 16.2 & 448,803 & 16.2 & 1.14 & 43,939 & 41,606 & .95 & 219,963 & 254,895 & 1.16 & 167,639 & 194,692 & 1.16 & 17,262 & 20,021 & 1.16 \\
\hline Totals & $3,152,441$ & 100 & $2,768,242$ & 100 & 1.14 & 469,674 & 513,911 & 1.09 & 889,192 & $1,046,886$ & 1.18 & 966,859 & $1,108,274$ & 1.15 & 442,517 & 483,370 & 1.09 \\
\hline \multicolumn{18}{|c|}{1999} \\
\hline 1 & 219,915 & 7.7 & 246,636 & 8.8 & .89 & 77,205 & 75,953 & .98 & 40,196 & 33,389 & .83 & 93,569 & 79,546 & .85 & 35,666 & 31,027 & .87 \\
\hline 2 & 85,121 & 3.0 & 114,635 & 4.1 & .74 & 26,588 & 22,574 & .85 & 35,230 & 24,622 & .70 & 42,404 & 30,645 & .72 & 10,413 & 7,280 & .70 \\
\hline 3 & $1,759,432$ & 61.5 & $1,594,865$ & 57.1 & 1.10 & 239,048 & 259,471 & 1.09 & 409,610 & 468,742 & 1.14 & 586,912 & 663,830 & 1.13 & 359,295 & 367,389 & 1.02 \\
\hline 4 & 331,370 & 11.6 & 378,827 & 13.6 & .87 & 26,988 & 28,555 & 1.06 & 95,372 & 80,905 & .85 & 219,918 & 189,915 & .86 & 36,549 & 31,995 & .88 \\
\hline 5 & 463,473 & 16.2 & 457,878 & 16.4 & 1.01 & 42,592 & 35,707 & .84 & 209,721 & 217,124 & 1.04 & 189,085 & 195,702 & 1.03 & 16,480 & 14,940 & .91 \\
\hline Totals & $2,859,311$ & 100 & $2,792,841$ & 100 & 1.02 & 412,421 & 422,260 & 1.02 & 790,129 & 824,782 & 1.04 & $1,131,888$ & $1,159,638$ & 1.02 & 458,403 & 452,631 & .99 \\
\hline \multicolumn{18}{|c|}{2000} \\
\hline 1 & 275,750 & 7.9 & 250,274 & 9.0 & 1.10 & 66,913 & 75,083 & 1.12 & 27,605 & 27,129 & .98 & 119,262 & 131,780 & 1.10 & 36,494 & 41,758 & 1.14 \\
\hline 2 & 112,448 & 3.2 & 116,186 & 4.2 & .97 & 26,875 & 30,136 & 1.12 & 25,159 & 21,843 & .87 & 52,818 & 50,443 & .96 & 11,334 & 10,026 & .88 \\
\hline 3 & $2,117,282$ & 60.4 & $1,584,541$ & 56.9 & 1.34 & 207,349 & 265,080 & 1.28 & 327,109 & 444,594 & 1.36 & 716,366 & 995,219 & 1.39 & 333,717 & 412,389 & 1.24 \\
\hline 4 & 472,688 & 13.5 & 374,945 & 13.5 & 1.26 & 20,792 & 30,969 & 1.49 & 70,750 & 86,909 & 1.23 & 250,894 & 316,092 & 1.26 & 32,509 & 38,718 & 1.19 \\
\hline 5 & 528,801 & 15.1 & 458,336 & 16.5 & 1.15 & 38,213 & 41,422 & 1.08 & 174,385 & 204,296 & 1.17 & 229,741 & 265,459 & 1.16 & 15,997 & 17,624 & 1.10 \\
\hline Totals & $3,506,969$ & 100 & $2,784,282$ & 100 & 1.26 & 360,142 & 442,690 & 1.23 & 625,008 & 784,771 & 1.26 & $1,369,081$ & $1,758,993$ & 1.28 & 430,051 & 520,515 & 1.21 \\
\hline
\end{tabular}


Table 3. Irrigation water use, acres irrigated, and irrigation application rate by system type for the Groundwater Management Districts within the Kansas High Plains, 1991-2003.-Continued

[Data from Kansas Water Office and Kansas Department of Agriculture (1993-2005). acre-ft/acre, acre-foot per acre]

\begin{tabular}{|c|c|c|c|c|c|c|c|c|c|c|c|c|c|c|c|c|c|}
\hline \multirow{2}{*}{$\begin{array}{c}\text { Ground- } \\
\text { water } \\
\text { Manage- } \\
\text { ment } \\
\text { District } \\
\text { (GMD) } \\
\text { number } \\
\text { (fig. 1) }\end{array}$} & \multicolumn{2}{|c|}{$\begin{array}{l}\text { Total irrigation water } \\
\text { use }\end{array}$} & \multicolumn{2}{|c|}{ Total acres irrigated } & \multirow{2}{*}{$\begin{array}{l}\text { Aver- } \\
\text { age } \\
\text { rate of } \\
\text { irri- } \\
\text { gation } \\
\text { (acre- } \\
\mathrm{ft} / \mathrm{yr} \text { ) }\end{array}$} & \multicolumn{3}{|c|}{ Flood irrigation } & \multicolumn{3}{|c|}{ Center pivot irrigation } & \multicolumn{3}{|c|}{$\begin{array}{l}\text { Center pivot irrigation with drop } \\
\text { nozzle }\end{array}$} & \multicolumn{3}{|c|}{ Other irrigation systems } \\
\hline & Acre-feet & $\begin{array}{l}\text { Percen- } \\
\text { tage of } \\
\text { total for } \\
\text { all GMDs }\end{array}$ & Acres & $\begin{array}{l}\text { Percen- } \\
\text { tage of } \\
\text { total for } \\
\text { all GMDs }\end{array}$ & & $\begin{array}{l}\text { Total acres } \\
\text { irrigated }\end{array}$ & $\begin{array}{l}\text { Water used } \\
\text { (acre-ft) }\end{array}$ & $\begin{array}{l}\text { Rate of } \\
\text { irri- } \\
\text { gation } \\
\text { (acre- } \\
\text { ft/acre) }\end{array}$ & $\begin{array}{l}\text { Total acres } \\
\text { irrigated }\end{array}$ & $\begin{array}{l}\text { Water used } \\
\text { (acre-ft) }\end{array}$ & $\begin{array}{c}\text { Rate of } \\
\text { irri- } \\
\text { gation } \\
\text { (acre- } \\
\text { ft/acre) }\end{array}$ & $\begin{array}{l}\text { Total acres } \\
\text { irrigated }\end{array}$ & $\begin{array}{l}\text { Water used } \\
\text { (acre-ft) }\end{array}$ & $\begin{array}{l}\text { Rate of } \\
\text { irri- } \\
\text { gation } \\
\text { (acre- } \\
\mathrm{ft} / \text { acre) }\end{array}$ & $\begin{array}{l}\text { Total acres } \\
\text { irrigated }\end{array}$ & $\begin{array}{c}\text { Water used } \\
\text { (acre-ft) }\end{array}$ & $\begin{array}{c}\text { Rate of } \\
\text { irri- } \\
\text { gation } \\
\text { (acre- } \\
\text { ft/acre) }\end{array}$ \\
\hline \multicolumn{18}{|c|}{2001} \\
\hline 1 & 226,216 & 7.0 & 237,016 & 8.7 & 0.95 & 52,491 & 53,405 & 1.02 & 29,663 & 26,016 & 0.88 & 120,284 & 115,239 & 0.96 & 34,578 & 31,556 & 0.91 \\
\hline 2 & 131,639 & 4.1 & 117,476 & 4.3 & 1.12 & 25,419 & 31,743 & 1.25 & 25,522 & 26,152 & 1.02 & 54,087 & 60,227 & 1.11 & 12,448 & 13,517 & 1.09 \\
\hline 3 & $1,889,226$ & 58.7 & $1,552,436$ & 56.7 & 1.22 & 163,657 & 187,146 & 1.14 & 277,253 & 338,657 & 1.22 & 771,727 & 974,471 & 1.26 & 339,799 & 388,952 & 1.14 \\
\hline 4 & 402,236 & 12.5 & 367,627 & 13.4 & 1.09 & 16,830 & 20,649 & 1.23 & 57,955 & 64,016 & 1.10 & 260,665 & 281,731 & 1.08 & 32,177 & 35,840 & 1.11 \\
\hline 5 & 567,745 & 17.6 & 462,003 & 16.9 & 1.23 & 39,014 & 41,734 & 1.07 & 143,220 & 176,108 & 1.23 & 258,986 & 325,155 & 1.26 & 20,783 & 24,748 & 1.19 \\
\hline Totals & $3,217,062$ & 100 & $2,736,558$ & 100 & 1.18 & 297,411 & 334,677 & 1.13 & 533,613 & 630,949 & 1.18 & $1,465,749$ & $1,756,823$ & 1.20 & 439,785 & 494,613 & 1.12 \\
\hline \multicolumn{18}{|c|}{2002} \\
\hline 1 & 286,707 & 7.5 & 243,617 & 8.7 & 1.18 & 51,732 & 61,436 & 1.19 & 23,716 & 26,216 & 1.11 & 123,104 & 147,330 & 1.20 & 45,065 & 51,725 & 1.15 \\
\hline 2 & 125,933 & 3.3 & 120,840 & 4.3 & 1.04 & 24,826 & 30,690 & 1.24 & 20,090 & 20,116 & 1.00 & 61,401 & 61,132 & 1.00 & 14,523 & 13,995 & .96 \\
\hline 3 & $2,357,519$ & 61.4 & $1,594,975$ & 57.0 & 1.48 & 150,509 & 234,006 & 1.55 & 238,835 & 347,155 & 1.45 & 818,106 & $1,238,698$ & 1.51 & 387,525 & 537,660 & 1.39 \\
\hline 4 & 500,716 & 13.0 & 374,371 & 13.4 & 1.34 & 14,672 & 22,270 & 1.52 & 50,587 & 66,332 & 1.31 & 275,019 & 368,947 & 1.34 & 34,093 & 43,167 & 1.27 \\
\hline 5 & 571,254 & 14.9 & 464,945 & 16.6 & 1.23 & 35,692 & 39,650 & 1.11 & 118,264 & 145,967 & 1.23 & 290,459 & 360,588 & 1.24 & 20,530 & 25,049 & 1.22 \\
\hline Totals & $3,842,129$ & 100 & $2,798,748$ & 100 & 1.37 & 277,431 & 388,052 & 1.40 & 451,492 & 605,786 & 1.34 & $1,568,089$ & $2,176,695$ & 1.39 & 501,736 & 671,596 & 1.34 \\
\hline \multicolumn{18}{|c|}{2003} \\
\hline 1 & 236,793 & 7.1 & 235,050 & 8.7 & 1.01 & 47,292 & 49,659 & 1.05 & 18,770 & 17,394 & .93 & 132,831 & 132,349 & 1.00 & 36,157 & 37,391 & 1.03 \\
\hline 2 & 138,811 & 4.1 & 121,404 & 4.5 & 1.14 & 22,520 & 29,566 & 1.31 & 19,362 & 21,060 & 1.09 & 63,854 & 70,842 & 1.11 & 15,668 & 17,343 & 1.11 \\
\hline 3 & 1,937,199 & 57.8 & $1,514,599$ & 56.0 & 1.28 & 112,449 & 144,707 & 1.29 & 215,483 & 266,573 & 1.24 & 842,524 & $1,094,106$ & 1.30 & 344,143 & 431,813 & 1.25 \\
\hline 4 & 460,830 & 13.8 & 375,994 & 13.9 & 1.23 & 11,610 & 16,227 & 1.40 & 49,425 & 57,226 & 1.16 & 283,226 & 349,235 & 1.23 & 31,733 & 38,142 & 1.20 \\
\hline 5 & 575,807 & 17.2 & 456,932 & 16.9 & 1.26 & 30,817 & 39,546 & 1.28 & 99,141 & 126,458 & 1.28 & 307,325 & 385,951 & 1.26 & 19,649 & 23,852 & 1.21 \\
\hline Totals & $3,349,440$ & 100 & $2,703,979$ & 100 & 1.24 & 224,688 & 279,705 & 1.24 & 402,181 & 488,711 & 1.22 & $1,629,760$ & $2,032,483$ & 1.25 & 447,350 & 548,541 & 1.23 \\
\hline
\end{tabular}


Table 4. Irrigation water use, acres irrigated, and irrigation application rate by crop type for the Groundwater Management Districts within the Kansas High Plains, $1991-2003$.

[Data from Kansas Water Office and Kansas Department of Agriculture (1993-2005). acre-ft/acre, acre-foot per acre]

\begin{tabular}{|c|c|c|c|c|c|c|c|c|c|c|c|c|c|c|c|c|c|c|}
\hline \multirow{2}{*}{$\begin{array}{c}\text { Ground- } \\
\text { water } \\
\text { Manage- } \\
\text { ment } \\
\text { District } \\
\text { number } \\
\text { (fig. 1) }\end{array}$} & \multicolumn{3}{|c|}{ Alfalfa } & \multicolumn{3}{|c|}{ Corn } & \multicolumn{3}{|c|}{ Grain sorghum } & \multicolumn{3}{|c|}{ Soybeans } & \multicolumn{3}{|c|}{ Wheat } & \multicolumn{3}{|c|}{ Other/Multiple Use } \\
\hline & $\begin{array}{c}\text { Total } \\
\text { acres } \\
\text { irrigated }\end{array}$ & $\begin{array}{l}\text { Irrigation } \\
\text { water } \\
\text { used } \\
\text { (acre- } \\
\text { feet) }\end{array}$ & $\begin{array}{l}\text { Rate of } \\
\text { irri- } \\
\text { gation } \\
\text { (acre- } \\
\text { ft/acre) }\end{array}$ & $\begin{array}{c}\text { Total } \\
\text { acres } \\
\text { irrigated }\end{array}$ & $\begin{array}{c}\text { Irrigation } \\
\text { waterused } \\
\text { (acre-feet) }\end{array}$ & $\begin{array}{l}\text { Rate of } \\
\text { irri- } \\
\text { gation } \\
\text { (acre- } \\
\text { ft/acre) }\end{array}$ & $\begin{array}{c}\text { Total } \\
\text { acres } \\
\text { irrigated }\end{array}$ & $\begin{array}{l}\text { Irrigation } \\
\text { water } \\
\text { used } \\
\text { (acre-feet) }\end{array}$ & $\begin{array}{l}\text { Rate of } \\
\text { irri- } \\
\text { gation } \\
\text { (acre- } \\
\text { ft/acre) }\end{array}$ & $\begin{array}{l}\text { Total } \\
\text { acres } \\
\text { irrigated }\end{array}$ & $\begin{array}{l}\text { Irrigation } \\
\text { water used } \\
\text { (acre-feet) }\end{array}$ & $\begin{array}{l}\text { Rate of } \\
\text { irri- } \\
\text { gation } \\
\text { (acre- } \\
\text { ft/acre) }\end{array}$ & $\begin{array}{c}\text { Total } \\
\text { acres } \\
\text { irrigated }\end{array}$ & $\begin{array}{l}\text { Irrigation } \\
\text { water } \\
\text { used } \\
\text { (acre- } \\
\text { feet) }\end{array}$ & $\begin{array}{l}\text { Rate of } \\
\text { irri- } \\
\text { gation } \\
\text { (acre- } \\
\text { ft/acre) }\end{array}$ & $\begin{array}{c}\text { Total acres } \\
\text { irrigated }\end{array}$ & $\begin{array}{l}\text { Irrigation } \\
\text { water used } \\
\text { (acre-feet) }\end{array}$ & $\begin{array}{l}\text { Rate of } \\
\text { irri- } \\
\text { gation } \\
\text { (acre- } \\
\text { ft/acre) }\end{array}$ \\
\hline \multicolumn{19}{|c|}{1991} \\
\hline 1 & 3,061 & 4,431 & 1.45 & 41,584 & 64,116 & 1.54 & 15,576 & 18,257 & 1.17 & 301 & 431 & 1.43 & 7,655 & 5,800 & 0.76 & 194,351 & 262,554 & 1.35 \\
\hline 2 & 1,311 & 1,173 & .90 & 22,721 & 29,650 & 1.30 & 7,304 & 5,589 & .77 & 13,733 & 15,487 & 1.13 & 721 & 418 & .58 & 52,043 & 60,286 & 1.16 \\
\hline 3 & 112,077 & 227,401 & 2.03 & 186,557 & 356,541 & 1.91 & 49,141 & 72,187 & 1.47 & 9,440 & 14,308 & 1.52 & 82,560 & 90,641 & 1.10 & $1,153,416$ & $1,899,155$ & 1.65 \\
\hline 4 & 6,383 & 9,317 & 1.46 & 140,359 & 212,020 & 1.51 & 23,474 & 28,699 & 1.22 & 5,604 & 6,630 & 1.18 & 15,816 & 11,209 & .71 & 168,473 & 210,980 & 1.25 \\
\hline 5 & 47,721 & 68,609 & 1.44 & 158,182 & 232,206 & 1.47 & 18,691 & 21,402 & 1.15 & 29,079 & 40,321 & 1.39 & 15,114 & 10,958 & .73 & 165,958 & 233,176 & 1.41 \\
\hline Totals & \multicolumn{17}{|c|}{1992} & 1.54 \\
\hline 1 & 2,828 & 4,524 & 1.60 & 48,455 & 62,838 & 1.30 & 12,940 & 11,602 & .90 & 916 & 1,003 & 1.10 & 7,974 & 5,652 & .71 & 171,469 & 184,524 & 1.08 \\
\hline 2 & 1,730 & 1,077 & .62 & 19,827 & 11,723 & .59 & 8,719 & 4,837 & .55 & 13,589 & 9,599 & .71 & 927 & 329 & .36 & 48,088 & 29,629 & .62 \\
\hline 3 & 104,396 & 175,814 & 1.68 & 217,375 & 336,529 & 1.55 & 42,784 & 39,958 & .93 & 10,676 & 10,342 & .97 & 79,339 & 81,315 & 1.02 & $1,125,514$ & $1,534,314$ & 1.36 \\
\hline 4 & 5,237 & 4,765 & .91 & 139,908 & 119,470 & .85 & 28,445 & 20,129 & .71 & 6,996 & 4,705 & .67 & 15,184 & 8,169 & .54 & 144,752 & 110,979 & .77 \\
\hline 5 & 42,745 & 37,283 & .87 & 180,103 & 152,136 & .84 & 18,262 & 9,483 & .52 & 21,307 & 16,609 & .78 & 14,586 & 7,734 & .53 & 141,746 & 107,162 & .76 \\
\hline Totals & 156,936 & 223,463 & 1.42 & 605,668 & 682,696 & 1.13 & 111,150 & 86,009 & .77 & 53,484 & 42,258 & .79 & 118,010 & 103,199 & .87 & $1,631,569$ & $1,966,608$ & 1.21 \\
\hline \multicolumn{19}{|c|}{1993} \\
\hline 1 & 2,034 & 2,608 & 1.28 & 58,762 & 64,761 & 1.10 & 9,997 & 7,270 & .73 & 67 & 17 & .26 & 5,459 & 2,409 & .44 & 146,727 & 120,534 & .82 \\
\hline 2 & 1,000 & 507 & .51 & 20,270 & 14,016 & .69 & 4,813 & 2,213 & .46 & 12,330 & 9,371 & .76 & 633 & 114 & .18 & 51,479 & 32,928 & .64 \\
\hline 3 & 103,698 & 162,041 & 1.56 & 259,940 & 390,866 & 1.50 & 26,218 & 21,496 & .82 & 6,799 & 6,715 & .99 & 68,829 & 55,768 & .81 & $1,084,912$ & $1,250,936$ & 1.15 \\
\hline 4 & 6,289 & 4,808 & .76 & 164,651 & 139,859 & .85 & 6,759 & 3,405 & .50 & 4,489 & 2,536 & .57 & 16,204 & 7,811 & .48 & 145,268 & 103,228 & .71 \\
\hline 5 & 40,900 & 28,089 & .69 & 184,608 & 158,642 & .86 & 12,528 & 5,227 & .42 & 23,824 & 18,912 & .79 & 11,746 & 3,497 & .30 & 136,724 & 94,107 & .69 \\
\hline All & 153,921 & 198,053 & 1.29 & 688,231 & 768,144 & 1.12 & 60,315 & 39,611 & .66 & 47,509 & 37,551 & .79 & 102,871 & 69,599 & .68 & $1,565,110$ & $1,601,733$ & 1.02 \\
\hline \multicolumn{19}{|c|}{1994} \\
\hline 1 & 3,015 & 3,990 & 1.32 & 50,703 & 73,305 & 1.45 & 10,029 & 8,526 & .85 & 317 & 403 & 1.27 & 4,566 & 2,477 & .54 & 168,363 & 193,220 & 1.15 \\
\hline 2 & 1,179 & 1,382 & 1.17 & 21,342 & 24,224 & 1.14 & 4,076 & 3,498 & .86 & 16,155 & 17,032 & 1.05 & 802 & 213 & .27 & 53,689 & 54,234 & 1.01 \\
\hline 3 & 109,133 & 199,017 & 1.82 & 277,076 & 464,174 & 1.68 & 20,143 & 20,990 & 1.04 & 6,735 & 9,086 & 1.35 & 59,655 & 56,857 & .95 & $1,082,823$ & $1,489,030$ & 1.38 \\
\hline 4 & 5,676 & 5,800 & 1.02 & 177,265 & 218,340 & 1.23 & 7,125 & 5,941 & .83 & 3,929 & 3,709 & .94 & 11,625 & 6,803 & .59 & 161,450 & 156,009 & .97 \\
\hline 5 & 41,386 & 56,319 & 1.36 & 189,124 & 264,327 & 1.40 & 11,889 & 11,158 & .94 & 29,119 & 38,466 & 1.32 & 7,308 & 5,613 & .77 & 149,196 & 189,901 & 1.27 \\
\hline Totals & 160,389 & 266,508 & 1.66 & 715,510 & $1,044,370$ & 1.46 & 53,262 & 50,113 & .94 & 56,255 & 68,696 & 1.22 & 83,956 & 71,963 & .86 & $1,615,521$ & $2,082,394$ & 1.29 \\
\hline \multicolumn{19}{|c|}{1995} \\
\hline 1 & 1,944 & 2,992 & 1.54 & 54,699 & 68,045 & 1.24 & 9,488 & 8,107 & .85 & 0 & 0 & .00 & 7,371 & 5,126 & .70 & 169,010 & 174,586 & 1.03 \\
\hline 2 & 1,178 & 717 & .61 & 20,084 & 19,902 & .99 & 7,060 & 4,822 & .68 & 15,803 & 14,032 & .89 & 1,227 & 393 & .32 & 52,462 & 45,075 & .86 \\
\hline 3 & 114,411 & 193,784 & 1.69 & 278,912 & 450,640 & 1.62 & 20,033 & 19,878 & .99 & 4,709 & 6,199 & 1.32 & 49,554 & 39,819 & .80 & $1,065,790$ & $1,399,593$ & 1.31 \\
\hline 4 & 7,402 & 7,129 & .96 & 158,766 & 195,690 & 1.23 & 8,364 & 7,067 & .84 & 3,576 & 3,495 & .98 & 17,480 & 8,001 & .46 & 159,671 & 140,396 & .88 \\
\hline 5 & 40,244 & 37,877 & .94 & 185,095 & 221,508 & 1.20 & 14,059 & 10,447 & .74 & 28,128 & 31,444 & 1.12 & 10,071 & 4,496 & .45 & 149,213 & 147,743 & .99 \\
\hline Totals & 165,179 & 242,499 & 1.47 & 697,556 & 955,785 & 1.37 & 59,004 & 50,321 & .85 & 52,216 & 55,170 & 1.06 & 85,703 & 57,835 & .67 & $1,596,146$ & $1,907,393$ & 1.19 \\
\hline
\end{tabular}


Table 4. Irrigation water use, acres irrigated, and irrigation application rate by crop type for the Groundwater Management Districts within the Kansas High Plains, 1991-2003.-Continued

[Data from Kansas Water Office and Kansas Department of Agriculture (1993-2005). acre-ft/acre, acre-foot per acre]

\begin{tabular}{|c|c|c|c|c|c|c|c|c|c|c|c|c|c|c|c|c|c|c|}
\hline \multirow{2}{*}{$\begin{array}{c}\text { Ground- } \\
\text { water } \\
\text { Manage- } \\
\text { ment } \\
\text { District } \\
\text { number } \\
\text { (fig. 1) }\end{array}$} & \multicolumn{3}{|c|}{ Alfalfa } & \multicolumn{3}{|c|}{ Corn } & \multicolumn{3}{|c|}{ Grain sorghum } & \multicolumn{3}{|c|}{ Soybeans } & \multicolumn{3}{|c|}{ Wheat } & \multicolumn{3}{|c|}{ Other/Multiple Use } \\
\hline & $\begin{array}{l}\text { Total } \\
\text { acres } \\
\text { irrigated }\end{array}$ & $\begin{array}{c}\text { Irrigation } \\
\text { water } \\
\text { used } \\
\text { (acre- } \\
\text { feet) }\end{array}$ & $\begin{array}{l}\text { Rate of } \\
\text { irri- } \\
\text { gation } \\
\text { (acre- } \\
\text { ft/acre) }\end{array}$ & $\begin{array}{l}\text { Total } \\
\text { acres } \\
\text { irrigated }\end{array}$ & $\begin{array}{c}\text { Irrigation } \\
\text { waterused } \\
\text { (acre-feet) }\end{array}$ & $\begin{array}{l}\text { Rate of } \\
\text { irri- } \\
\text { gation } \\
\text { (acre- } \\
\text { tt/acre) }\end{array}$ & $\begin{array}{c}\text { Total } \\
\text { acres } \\
\text { irrigated }\end{array}$ & $\begin{array}{l}\text { Irrigation } \\
\text { water } \\
\text { used } \\
\text { (acre-feet) }\end{array}$ & $\begin{array}{l}\text { Rate of } \\
\text { irri- } \\
\text { gation } \\
\text { (acre- } \\
\text { ft/acre) }\end{array}$ & $\begin{array}{c}\text { Total } \\
\text { acres } \\
\text { irrigated }\end{array}$ & $\begin{array}{l}\text { Irrigation } \\
\text { water used } \\
\text { (acre-feet) }\end{array}$ & $\begin{array}{l}\text { Rate of } \\
\text { irri- } \\
\text { gation } \\
\text { (acre- } \\
\text { ft/acre) }\end{array}$ & $\begin{array}{c}\text { Total } \\
\text { acres } \\
\text { irrigated }\end{array}$ & $\begin{array}{l}\text { Irrigation } \\
\text { water } \\
\text { used } \\
\text { (acre- } \\
\text { feet) }\end{array}$ & $\begin{array}{l}\text { Rate of } \\
\text { irri- } \\
\text { gation } \\
\text { (acre- } \\
\text { ft/acre) }\end{array}$ & $\begin{array}{l}\text { Total acres } \\
\text { irrigated }\end{array}$ & $\begin{array}{l}\text { Irrigation } \\
\text { water used } \\
\text { (acre-feet) }\end{array}$ & $\begin{array}{c}\text { Rate of } \\
\text { irri- } \\
\text { gation } \\
\text { (acre- } \\
\mathrm{ft} / \text { acre) }\end{array}$ \\
\hline \multicolumn{19}{|c|}{1996} \\
\hline 1 & 2,902 & 3,458 & 1.19 & 66,332 & 82,493 & 1.24 & 9,267 & 6,801 & 0.73 & 118 & 82 & 0.69 & 4,597 & 2,082 & 0.45 & 159,426 & 155,127 & 0.97 \\
\hline 2 & 1,195 & 847 & .71 & 24,474 & 24,680 & 1.01 & 5,578 & 3,615 & .65 & 15,063 & 11,988 & .80 & 1,490 & 489 & .33 & 55,797 & 45,027 & .81 \\
\hline 3 & 108,819 & 168,783 & 1.55 & 331,052 & 489,911 & 1.48 & 20,459 & 16,720 & .82 & 4,855 & 4,083 & .84 & 36,924 & 30,617 & .83 & $1,022,148$ & $1,239,878$ & 1.21 \\
\hline 4 & 7,200 & 6,499 & .90 & 204,159 & 224,076 & 1.10 & 6,333 & 4,133 & .65 & 2,504 & 2,264 & .90 & 13,580 & 7,217 & .53 & 136,865 & 120,498 & .88 \\
\hline 5 & 37,692 & 29,566 & .78 & 210,359 & 200,650 & .95 & 14,229 & 8,771 & .62 & 31,627 & 25,044 & .79 & 5,985 & 2,522 & .42 & 131,487 & 105,197 & .80 \\
\hline Totals & 157,808 & 209,153 & 1.33 & 836,376 & $1,021,810$ & 1.22 & 55,866 & 40,040 & .72 & 54,167 & 43,461 & .80 & 62,576 & 42,927 & .69 & $1,505,723$ & $1,665,727$ & 1.11 \\
\hline \multicolumn{19}{|c|}{1997} \\
\hline 1 & 2,967 & 3,472 & 1.17 & 69,309 & 76,172 & 1.10 & 7,183 & 4,226 & .59 & 544 & 416 & .76 & 9,338 & 5,386 & .58 & 157,156 & 136,200 & .87 \\
\hline 2 & 1,376 & 887 & .64 & 26,924 & 21,082 & .78 & 3,876 & 2,174 & .56 & 21,919 & 15,898 & .73 & 1,401 & 424 & .30 & 51,275 & 35,993 & .70 \\
\hline 3 & 124,470 & 202,750 & 1.63 & 381,109 & 506,695 & 1.33 & 19,094 & 15,927 & .83 & 6,724 & 7,227 & 1.07 & 38,921 & 29,771 & .76 & $1,006,112$ & $1,089,424$ & 1.08 \\
\hline 4 & 8,309 & 9,095 & 1.09 & 222,164 & 265,040 & 1.19 & 5,745 & 4,360 & .76 & 6,348 & 6,523 & 1.03 & 13,982 & 8,530 & .61 & 125,387 & 119,518 & .95 \\
\hline 5 & 37,009 & 27,488 & .74 & 200,050 & 170,569 & .85 & 10,588 & 6,042 & .57 & 54,749 & 42,507 & .78 & 6,481 & 2,322 & .36 & 130,161 & 92,926 & .71 \\
\hline Totals & 174,131 & 243,692 & 1.40 & 899,556 & $1,039,558$ & 1.16 & 46,486 & 32,729 & .70 & 90,284 & 72,571 & .80 & 70,123 & 46,433 & .66 & $1,470,091$ & $1,474,061$ & 1.00 \\
\hline \multicolumn{19}{|c|}{1998} \\
\hline 1 & 2,859 & 3,486 & 1.22 & 69,902 & 79,262 & 1.13 & 2,960 & 1,659 & .56 & 1,362 & 1,027 & .75 & 6,647 & 4,429 & .67 & 161,153 & 133,526 & .83 \\
\hline 2 & 1,656 & 1,527 & .92 & 27,370 & 25,714 & .94 & 3,698 & 2,576 & .70 & 24,134 & 23,689 & .98 & 1,012 & 476 & .47 & 52,571 & 46,844 & .89 \\
\hline 3 & 136,561 & 233,416 & 1.71 & 410,369 & 602,187 & 1.47 & 9,130 & 6,924 & .76 & 12,184 & 15,819 & 1.30 & 33,583 & 25,253 & .75 & 981,576 & $1,056,562$ & 1.08 \\
\hline 4 & 9,299 & 10,419 & 1.12 & 219,731 & 240,991 & 1.10 & 4,483 & 2,998 & .67 & 10,096 & 8,950 & .89 & 9,518 & 5,203 & .55 & 127,585 & 108,291 & .85 \\
\hline 5 & 36,064 & 41,255 & 1.14 & 203,016 & 245,091 & 1.21 & 7,602 & 5,766 & .76 & 66,447 & 78,408 & 1.18 & 5,238 & 1,969 & .38 & 130,436 & 138,726 & 1.06 \\
\hline Totals & 186,439 & 290,103 & 1.56 & 930,388 & $1,193,245$ & 1.28 & 27,873 & 19,923 & .71 & 114,223 & 127,893 & 1.12 & 55,998 & 37,330 & .67 & $1,453,321$ & $1,483,949$ & 1.02 \\
\hline \multicolumn{19}{|c|}{1999} \\
\hline 1 & 2,555 & 3,430 & 1.34 & 68,483 & 74,841 & 1.09 & 3,181 & 2,672 & .84 & 1,462 & 1,612 & 1.10 & 5,492 & 2,454 & .45 & 165,463 & 134,906 & .82 \\
\hline 2 & 1,248 & 883 & .71 & 30,616 & 22,628 & .74 & 2,993 & 1,768 & .59 & 29,126 & 22,773 & .78 & 684 & 239 & .35 & 49,968 & 36,830 & .74 \\
\hline 3 & 130,184 & 185,079 & 1.42 & 422,801 & 565,797 & 1.34 & 11,114 & 8,320 & .75 & 21,090 & 21,963 & 1.04 & 37,965 & 23,033 & .61 & 971,711 & 955,240 & .98 \\
\hline 4 & 9,552 & 8,836 & .93 & 215,832 & 209,552 & .97 & 3,479 & 2,196 & .63 & 12,014 & 9,166 & .76 & 11,810 & 6,000 & .51 & 126,140 & 95,620 & .76 \\
\hline 5 & 36,481 & 32,904 & .90 & 197,704 & 213,070 & 1.08 & 6,840 & 5,358 & .78 & 72,459 & 80,734 & 1.11 & 9,279 & 4,386 & .47 & 135,115 & 127,021 & .94 \\
\hline Totals & 180,020 & 231,132 & 1.28 & 935,436 & $1,085,888$ & 1.16 & 27,607 & 20,314 & .74 & 136,151 & 136,248 & 1.00 & 65,230 & 36,112 & .55 & $1,448,397$ & $1,349,617$ & .93 \\
\hline \multicolumn{19}{|c|}{2000} \\
\hline 1 & 2,266 & 3,117 & 1.38 & 68,448 & 94,918 & 1.39 & 2,494 & 2,221 & .89 & 1,600 & 1,499 & .94 & 6,651 & 4,168 & .63 & 168,815 & 169,827 & 1.01 \\
\hline 2 & 918 & 934 & 1.02 & 28,112 & 25,284 & .90 & 2,816 & 1,929 & .69 & 33,760 & 36,285 & 1.07 & 729 & 250 & .34 & 49,851 & 47,766 & .96 \\
\hline 3 & 122,033 & 222,914 & 1.83 & 454,130 & 720,060 & 1.59 & 9,357 & 8,154 & .87 & 25,365 & 36,181 & 1.43 & 37,045 & 28,325 & .76 & 936,611 & $1,101,647$ & 1.18 \\
\hline 4 & 7,897 & 9,928 & 1.26 & 215,444 & 298,766 & 1.39 & 2,526 & 2,126 & .84 & 15,063 & 18,755 & 1.25 & 9,165 & 5,391 & .59 & 124,850 & 137,721 & 1.10 \\
\hline 5 & 30,025 & 34,944 & 1.16 & 207,135 & 243,038 & 1.17 & 6,149 & 5,302 & .86 & 73,072 & 91,160 & 1.25 & 7,934 & 4,503 & .57 & 134,021 & 149,854 & 1.12 \\
\hline Totals & 163,139 & 271,837 & 1.67 & 973,269 & $1,382,066$ & 1.42 & 23,342 & 19,732 & .85 & 148,860 & 183,880 & 1.24 & 61,524 & 42,637 & .69 & $1,414,148$ & $1,606,815$ & 1.14 \\
\hline
\end{tabular}


Table 4. Irrigation water use, acres irrigated, and irrigation application rate by crop type for the Groundwater Management Districts within the Kansas High Plains,

1991-2003.-Continued

[Data from Kansas Water Office and Kansas Department of Agriculture (1993-2005). acre-ft/acre, acre-foot per acre]

\begin{tabular}{|c|c|c|c|c|c|c|c|c|c|c|c|c|c|c|c|c|c|c|}
\hline \multirow{2}{*}{$\begin{array}{c}\text { Ground- } \\
\text { water } \\
\text { Manage- } \\
\text { ment } \\
\text { District } \\
\text { number } \\
\text { (fig. 1) }\end{array}$} & \multicolumn{3}{|c|}{ Alfalfa } & \multicolumn{3}{|c|}{ Corn } & \multicolumn{3}{|c|}{ Grain sorghum } & \multicolumn{3}{|c|}{ Soybeans } & \multicolumn{3}{|c|}{ Wheat } & \multicolumn{3}{|c|}{ Other/Multiple Use } \\
\hline & $\begin{array}{l}\text { Total } \\
\text { acres } \\
\text { irrigated }\end{array}$ & $\begin{array}{l}\text { Irrigation } \\
\text { water } \\
\text { used } \\
\text { (acre- } \\
\text { feet) }\end{array}$ & $\begin{array}{l}\text { Rate of } \\
\text { irri- } \\
\text { gation } \\
\text { (acre- } \\
\text { ft/acre) }\end{array}$ & $\begin{array}{l}\text { Total } \\
\text { acres } \\
\text { irrigated }\end{array}$ & $\begin{array}{c}\text { Irrigation } \\
\text { waterused } \\
\text { (acre-feet) }\end{array}$ & $\begin{array}{l}\text { Rate of } \\
\text { irri- } \\
\text { gation } \\
\text { (acre- } \\
\text { ft/acre) }\end{array}$ & $\begin{array}{l}\text { Total } \\
\text { acres } \\
\text { irrigated }\end{array}$ & $\begin{array}{l}\text { Irrigation } \\
\text { water } \\
\text { used } \\
\text { (acre-feet) }\end{array}$ & $\begin{array}{l}\text { Rate of } \\
\text { irri- } \\
\text { gation } \\
\text { (acre- } \\
\text { ft/acre) }\end{array}$ & $\begin{array}{l}\text { Total } \\
\text { acres } \\
\text { irrigated }\end{array}$ & $\begin{array}{l}\text { Irrigation } \\
\text { water used } \\
\text { (acre-feet) }\end{array}$ & $\begin{array}{l}\text { Rate of } \\
\text { irri- } \\
\text { gation } \\
\text { (acre- } \\
\mathrm{ft} / \text { acre) }\end{array}$ & $\begin{array}{c}\text { Total } \\
\text { acres } \\
\text { irrigated }\end{array}$ & $\begin{array}{l}\text { Irrigation } \\
\text { water } \\
\text { used } \\
\text { (acre- } \\
\text { feet) }\end{array}$ & $\begin{array}{l}\text { Rate of } \\
\text { irri- } \\
\text { gation } \\
\text { (acre- } \\
\text { ft/acre) }\end{array}$ & $\begin{array}{c}\text { Total acres } \\
\text { irrigated }\end{array}$ & $\begin{array}{c}\text { Irrigation } \\
\text { water used } \\
\text { (acre-feet) }\end{array}$ & $\begin{array}{l}\text { Rate of } \\
\text { irri- } \\
\text { gation } \\
\text { (acre- } \\
\mathrm{ft} / \text { acre) }\end{array}$ \\
\hline \multicolumn{19}{|c|}{2001} \\
\hline 1 & 2,380 & 4,181 & 1.76 & 54,499 & 66,954 & 1.23 & 5,128 & 3,636 & 0.71 & 1,189 & 1,203 & 1.01 & 5,351 & 2,524 & 0.47 & 168,469 & 147,718 & 0.88 \\
\hline 2 & 1,365 & 1,089 & .80 & 30,904 & 34,676 & 1.12 & 3,134 & 2,722 & .87 & 31,783 & 36,951 & 1.16 & 1,194 & 663 & .55 & 49,096 & 55,537 & 1.13 \\
\hline 3 & 144,614 & 240,336 & 1.66 & 407,355 & 584,300 & 1.43 & 11,656 & 9,978 & .86 & 30,733 & 43,979 & 1.43 & 53,006 & 36,850 & .70 & 905,072 & 973,783 & 1.08 \\
\hline 4 & 8,702 & 11,094 & 1.27 & 185,803 & 233,083 & 1.25 & 6,159 & 5,871 & .95 & 19,852 & 21,553 & 1.09 & 12,662 & 6,726 & .53 & 134,449 & 123,908 & .92 \\
\hline 5 & 32,947 & 39,317 & 1.19 & 201,073 & 259,856 & 1.29 & 7,112 & 6,394 & .90 & 67,948 & 90,203 & 1.33 & 9,696 & 6,164 & .64 & 143,227 & 165,810 & 1.16 \\
\hline Totals & 190,008 & 296,017 & 1.56 & 879,634 & $1,178,869$ & 1.34 & 33,189 & 28,601 & .86 & 151,505 & 193,889 & 1.28 & 81,909 & 52,927 & .65 & $1,400,313$ & $1,466,756$ & 1.05 \\
\hline \multicolumn{19}{|c|}{2002} \\
\hline 1 & 2,521 & 3,996 & 1.58 & 47,875 & 75,844 & 1.58 & 4,553 & 5,068 & 1.11 & 1,520 & 1,550 & 1.02 & 5,613 & 4,289 & .76 & 181,535 & 195,960 & 1.08 \\
\hline 2 & 1,495 & 1,321 & .88 & 35,974 & 38,735 & 1.08 & 3,356 & 2,523 & .75 & 29,202 & 31,773 & 1.09 & 635 & 408 & .64 & 50,178 & 51,173 & 1.02 \\
\hline 3 & 159,240 & 298,900 & 1.88 & 382,199 & 673,482 & 1.76 & 18,561 & 18,252 & .98 & 26,349 & 42,690 & 1.62 & 40,498 & 33,603 & .83 & 968,128 & $1,290,592$ & 1.33 \\
\hline 4 & 8,801 & 13,601 & 1.55 & 178,199 & 262,899 & 1.48 & 4,393 & 4,553 & 1.04 & 18,856 & 26,376 & 1.40 & 8,869 & 6,056 & .68 & 155,253 & 187,230 & 1.21 \\
\hline 5 & 42,363 & 54,769 & 1.29 & 201,842 & 265,484 & 1.32 & 8,081 & 7,921 & .98 & 67,327 & 86,427 & 1.28 & 9,024 & 5,536 & .61 & 136,308 & 151,117 & 1.11 \\
\hline Totals & 214,420 & 372,587 & 1.74 & 846,089 & $1,316,444$ & 1.56 & 38,944 & 38,317 & .98 & 143,254 & 188,816 & 1.32 & 64,639 & 49,892 & .77 & $1,491,402$ & $1,876,072$ & 1.26 \\
\hline \multicolumn{19}{|c|}{2003} \\
\hline 1 & 2,260 & 3,237 & 1.43 & 31,591 & 45,476 & 1.44 & 5,649 & 4,257 & .75 & 839 & 778 & .93 & 10,641 & 5,751 & .54 & 184,070 & 177,294 & .96 \\
\hline 2 & 1,363 & 930 & .68 & 43,078 & 50,765 & 1.18 & 4,352 & 3,659 & .84 & 24,628 & 29,815 & 1.21 & 594 & 251 & .42 & 47,389 & 53,390 & 1.13 \\
\hline 3 & 156,530 & 267,136 & 1.71 & 331,063 & 503,489 & 1.52 & 28,148 & 25,065 & .89 & 28,668 & 38,911 & 1.36 & 49,694 & 36,793 & .74 & 920,496 & $1,065,806$ & 1.16 \\
\hline 4 & 10,240 & 13,600 & 1.33 & 136,095 & 198,494 & 1.46 & 8,313 & 8,448 & 1.02 & 15,726 & 19,700 & 1.25 & 21,318 & 13,773 & .65 & 184,302 & 206,816 & 1.12 \\
\hline 5 & 44,433 & 50,458 & 1.14 & 192,626 & 266,588 & 1.38 & 16,136 & 16,735 & 1.04 & 57,229 & 77,069 & 1.35 & 12,339 & 7,157 & .58 & 134,169 & 157,801 & 1.18 \\
\hline Totals & 214,826 & 335,361 & 1.56 & 734,453 & $1,064,812$ & 1.45 & 62,598 & 58,164 & .93 & 127,090 & 166,273 & 1.31 & 94,586 & 63,725 & .67 & $1,470,426$ & $1,661,107$ & 1.13 \\
\hline
\end{tabular}


Table 5. Average regional precipitation and irrigation water use in the Groundwater Management Districts within the Kansas High Plains, 1991-2003.

[Precipitation data from National Oceanic and Atmospheric Administration (1895-2005).Water-use data from Kansas Water Office and Kansas Department of Agriculture (1993-2005)]

\begin{tabular}{|c|c|c|c|}
\hline \multirow{2}{*}{$\begin{array}{l}\text { Groundwater } \\
\text { Management } \\
\text { District (GMD) } \\
\text { number (fig. 1) }\end{array}$} & \multirow{2}{*}{$\begin{array}{l}\text { Average } \\
\text { regional } \\
\text { precipitation } \\
\text { (inches) }\end{array}$} & \multicolumn{2}{|c|}{ Total irrigation water use } \\
\hline & & Acre-feet & $\begin{array}{c}\text { Percentage of } \\
\text { total for all } \\
\text { GMDs }\end{array}$ \\
\hline \multicolumn{4}{|c|}{1991} \\
\hline 1 & 19.84 & 355,589 & 8.4 \\
\hline 2 & 22.72 & 112,604 & 2.7 \\
\hline 3 & 18.19 & $2,660,233$ & 63.1 \\
\hline 4 & 22.67 & 478,854 & 11.4 \\
\hline 5 & 22.72 & 606,672 & 14.4 \\
\hline Totals & & $4,213,952$ & 100 \\
\hline \multicolumn{4}{|c|}{1992} \\
\hline 1 & 24.16 & 270,143 & 8.7 \\
\hline 2 & 31.96 & 57,195 & 1.8 \\
\hline 3 & 19.69 & $2,178,273$ & 70.2 \\
\hline 4 & 25.39 & 268,217 & 8.6 \\
\hline 5 & 31.96 & 330,406 & 10.6 \\
\hline Totals & & $3,104,234$ & 100 \\
\hline \multicolumn{4}{|c|}{1993} \\
\hline 1 & 28.72 & 197,600 & 7.3 \\
\hline 2 & 34.08 & 59,150 & 2.2 \\
\hline 3 & 24.11 & $1,887,823$ & 69.6 \\
\hline 4 & 30.09 & 261,648 & 9.6 \\
\hline 5 & 34.08 & 308,474 & 11.4 \\
\hline Totals & & $2,714,695$ & 100 \\
\hline \multicolumn{4}{|c|}{1994} \\
\hline 1 & 18.83 & 281,921 & 7.9 \\
\hline 2 & 22.06 & 100,583 & 2.8 \\
\hline 3 & 18.87 & $2,239,152$ & 62.5 \\
\hline 4 & 20.70 & 396,603 & 11.1 \\
\hline 5 & 22.06 & 565,785 & 15.8 \\
\hline Totals & & $3,584,044$ & 100 \\
\hline \multicolumn{4}{|c|}{1995} \\
\hline 1 & 21.55 & 258,857 & 7.9 \\
\hline 2 & 31.66 & 84,940 & 2.6 \\
\hline 3 & 18.45 & $2,109,913$ & 64.5 \\
\hline 4 & 22.90 & 361,778 & 11.1 \\
\hline 5 & 31.66 & 453,515 & 13.9 \\
\hline Totals & & $3,269,003$ & 100 \\
\hline \multicolumn{4}{|c|}{1996} \\
\hline 1 & 25.40 & 250,042 & 8.3 \\
\hline 2 & 32.01 & 86,645 & 2.9 \\
\hline 3 & 25.58 & $1,949,992$ & 64.5 \\
\hline 4 & 25.85 & 364,688 & 12.1 \\
\hline 5 & 32.01 & 371,749 & 12.3 \\
\hline Totals & & $3,023,116$ & 100 \\
\hline
\end{tabular}


Table 5. Average regional precipitation and irrigation water use in the Groundwater Management Districts within the Kansas High Plains, 1991-2003-Continued

[Precipitation data from National Oceanic and Atmospheric Administration (1895-2004).Water-use data from Kansas Water Office and Kansas Department of Agriculture (1993-2005)]

\begin{tabular}{|c|c|c|c|}
\hline \multirow{2}{*}{$\begin{array}{c}\text { Groundwater } \\
\text { Management } \\
\text { District (GMD) } \\
\text { number } \\
\text { (fig. 1) }\end{array}$} & \multirow[b]{2}{*}{$\begin{array}{l}\text { Average regional } \\
\text { precipitation } \\
\text { (inches) }\end{array}$} & \multicolumn{2}{|c|}{ Total irrigation water use } \\
\hline & & Acre-feet & $\begin{array}{c}\text { Percentage of } \\
\text { total for all } \\
\text { GMDs }\end{array}$ \\
\hline \multicolumn{4}{|c|}{1997} \\
\hline 1 & 23.51 & 225,872 & 7.8 \\
\hline 2 & 34.39 & 76,457 & 2.6 \\
\hline 3 & 23.68 & $1,851,794$ & 63.7 \\
\hline 4 & 19.98 & 413,066 & 14.2 \\
\hline 5 & 34.39 & 341,855 & 11.8 \\
\hline Totals & & $2,909,044$ & 100 \\
\hline \multicolumn{4}{|c|}{1998} \\
\hline 1 & 21.11 & 223,389 & 7.1 \\
\hline 2 & 30.14 & 100,825 & 3.2 \\
\hline 3 & 20.89 & $1,940,161$ & 61.6 \\
\hline 4 & 20.87 & 376,852 & 12.0 \\
\hline 5 & 30.14 & 511,214 & 16.2 \\
\hline Totals & & $3,152,441$ & 100 \\
\hline \multicolumn{4}{|c|}{1999} \\
\hline 1 & 21.42 & 219,915 & 7.7 \\
\hline 2 & 32.17 & 85,121 & 3.0 \\
\hline 3 & 20.36 & $1,759,432$ & 61.5 \\
\hline 4 & 21.73 & 331,370 & 11.6 \\
\hline 5 & 32.17 & 463,473 & 16.2 \\
\hline Totals & & $2,859,311$ & 100 \\
\hline \multicolumn{4}{|c|}{2000} \\
\hline 1 & 19.64 & 275,750 & 7.9 \\
\hline 2 & 32.76 & 112,448 & 3.2 \\
\hline 3 & 18.82 & $2,117,282$ & 60.4 \\
\hline 4 & 17.38 & 472,688 & 13.5 \\
\hline 5 & 32.76 & 528,801 & 15.1 \\
\hline Totals & & $3,506,969$ & 100 \\
\hline \multicolumn{4}{|c|}{2001} \\
\hline 1 & 19.93 & 226,216 & 7.0 \\
\hline 2 & 24.87 & 131,639 & 4.1 \\
\hline 3 & 18.45 & $1,889,226$ & 58.7 \\
\hline 4 & 20.48 & 402,236 & 12.5 \\
\hline 5 & 24.87 & 567,745 & 17.6 \\
\hline Totals & & $3,217,062$ & 100 \\
\hline \multicolumn{4}{|c|}{2002} \\
\hline 1 & 13.25 & 286,707 & 7.5 \\
\hline 2 & 29.41 & 125,933 & 3.3 \\
\hline 3 & 13.43 & $2,357,519$ & 61.4 \\
\hline 4 & 11.46 & 500,716 & 13.0 \\
\hline 5 & 29.41 & 571,254 & 14.9 \\
\hline Totals & & $3,842,129$ & 100 \\
\hline
\end{tabular}


Table 5. Average regional precipitation and irrigation water use in the Groundwater Management Districts within the Kansas High Plains, 1991-2003-Continued

[Precipitation data from National Oceanic and Atmospheric Administration (1895-2004).Water-use data from Kansas Water Office and Kansas Department of Agriculture (1993-2005)]

\begin{tabular}{cccc}
\hline $\begin{array}{c}\text { Groundwater } \\
\text { Management } \\
\text { District (GMD) } \\
\text { number } \\
\text { (fig. 1) }\end{array}$ & $\begin{array}{c}\text { Average regional } \\
\text { precipitation } \\
\text { (inches) }\end{array}$ & Acre-feet & $\begin{array}{c}\text { Percentage of } \\
\text { total for all } \\
\text { GMDs }\end{array}$ \\
\cline { 3 - 4 } & & & \\
1 & 17.04 & 236,793 & 7.1 \\
2 & 28.41 & 138,811 & 4.1 \\
3 & 17.98 & $1,937,199$ & 57.8 \\
4 & 16.06 & 460,830 & 13.8 \\
5 & 28.41 & 575,807 & 17.2 \\
Totals & & $3,349,440$ & 100 \\
\hline
\end{tabular}


Table 6. Trends in precipitation by meteorological regions in Kansas, 1895-2003.

[Trend analysis from Kendall and Gibbons (1990). Shading indicates significant trend at the 95-percent confidence interval]

\begin{tabular}{|c|c|c|c|c|}
\hline $\begin{array}{l}\text { Region } \\
\text { (fig. 1) }\end{array}$ & Tau & $p$ & Slope & $\begin{array}{l}\text { Median } \\
\text { (inches) }\end{array}$ \\
\hline \multicolumn{5}{|c|}{$1895-2003$} \\
\hline 1 & -0.014 & 0.836 & -0.003 & 22.55 \\
\hline 2 & .033 & .615 & .007 & 26.24 \\
\hline 3 & .038 & .558 & .015 & 33.71 \\
\hline 4 & .016 & .812 & .004 & 19.54 \\
\hline 5 & .099 & .127 & .027 & 26.45 \\
\hline 6 & .052 & .424 & .019 & 35.97 \\
\hline 7 & .068 & .298 & .013 & 18.48 \\
\hline 8 & .111 & .087 & .029 & 26.92 \\
\hline 9 & .125 & .054 & .045 & 37.21 \\
\hline \multicolumn{5}{|c|}{ 1950-2003 } \\
\hline 1 & .094 & .321 & .040 & 20.54 \\
\hline 2 & .013 & .899 & .005 & 27.37 \\
\hline 3 & .078 & .408 & .065 & 34.81 \\
\hline 4 & .066 & .483 & .028 & 19.88 \\
\hline 5 & .088 & .351 & .057 & 28.69 \\
\hline 6 & .097 & .303 & .073 & 36.38 \\
\hline 7 & .091 & .336 & .036 & 18.88 \\
\hline 8 & .185 & .185 & .101 & 28.16 \\
\hline 9 & .185 & .049 & .159 & 38.65 \\
\hline \multicolumn{5}{|c|}{ 1970-2003 } \\
\hline 1 & -.057 & .646 & -.026 & 21.01 \\
\hline 2 & 0 & 1 & 0 & 27.03 \\
\hline 3 & -.015 & .917 & -.009 & 35.33 \\
\hline 4 & .066 & .594 & .035 & 19.95 \\
\hline 5 & .020 & .882 & .029 & 28.69 \\
\hline 6 & .055 & .656 & .062 & 36.93 \\
\hline 7 & .075 & .543 & .049 & 18.88 \\
\hline 8 & .187 & .123 & .128 & 29.09 \\
\hline 9 & .070 & .573 & .049 & 39.23 \\
\hline \multicolumn{5}{|c|}{ 1980-2003 } \\
\hline 1 & -.228 & .124 & -.169 & 21.01 \\
\hline 2 & -.043 & .785 & -.069 & 26.90 \\
\hline 3 & -.116 & .442 & -.179 & 36.43 \\
\hline 4 & -.014 & .941 & -.014 & 20.08 \\
\hline 5 & .007 & .980 & .013 & 28.93 \\
\hline 6 & -.080 & .602 & -.122 & 37.41 \\
\hline 7 & .004 & 1 & .003 & 19.29 \\
\hline 8 & .174 & .244 & .130 & 29.32 \\
\hline 9 & .007 & .980 & .005 & 39.86 \\
\hline \multicolumn{5}{|c|}{ 1991-2003 } \\
\hline 1 & -.564 & .009 & -.839 & 20.87 \\
\hline 2 & -.180 & .428 & -.503 & 26.24 \\
\hline 3 & -.231 & .300 & -.749 & 34.99 \\
\hline 4 & -.436 & .044 & -.672 & 21.11 \\
\hline 5 & -.308 & .160 & -.825 & 31.17 \\
\hline 6 & -.179 & .428 & -.592 & 37.28 \\
\hline 7 & -.320 & .142 & -.390 & 18.87 \\
\hline 8 & -.026 & .951 & -.141 & 31.66 \\
\hline 9 & -.205 & .360 & -.556 & 40.02 \\
\hline
\end{tabular}


Table 7. Trends in precipitation and irrigation by system type, crop type, and prorated crop type for Groundwater Management District 1, west-central Kansas, $1991-2003$.

[Data from Kansas Water Office and Kansas Department of Agriculture (1993-2005). acre-ft, acre-feet; acre-ft/acre, acre-foot per acre. Blue shading indicates trend is significant at 95-percent level (p $\leq$ 0.05). Green shading indicates trend is significant at 90 -percent level $(\mathrm{p} \leq 0.10)$. NA, data not available]

\begin{tabular}{|c|c|c|c|c|c|c|c|c|c|c|c|c|c|c|c|c|}
\hline \multirow[b]{2}{*}{ Year } & \multirow[b]{2}{*}{$\begin{array}{l}\text { Meteor- } \\
\text { ological } \\
\text { region } 4 \\
\text { precipi- } \\
\text { tation } \\
\text { (inches) }\end{array}$} & \multirow[b]{2}{*}{$\begin{array}{c}\text { Total } \\
\text { acres } \\
\text { irrigated }\end{array}$} & \multirow[b]{2}{*}{$\begin{array}{c}\text { Total } \\
\text { irrigation } \\
\text { water use } \\
\text { (acre-ft) }\end{array}$} & \multirow[b]{2}{*}{$\begin{array}{c}\text { Average } \\
\text { rate of } \\
\text { irriga- } \\
\text { tion } \\
\text { (acre- } \\
\text { ft/acre) }\end{array}$} & \multicolumn{3}{|c|}{ Flood irrigation } & \multicolumn{3}{|c|}{ Center pivot irrigation } & \multicolumn{3}{|c|}{$\begin{array}{l}\text { Center pivot irrigation with drop } \\
\text { nozzle }\end{array}$} & \multicolumn{3}{|c|}{ Alfalfa } \\
\hline & & & & & $\begin{array}{c}\text { Total } \\
\text { acres } \\
\text { irrigated }\end{array}$ & $\begin{array}{c}\text { Water } \\
\text { used } \\
\text { (acre-ft) }\end{array}$ & $\begin{array}{l}\text { Rate of } \\
\text { irriga- } \\
\text { tion } \\
\text { (acre- } \\
\text { ft/acre) }\end{array}$ & $\begin{array}{l}\text { Total acres } \\
\text { irrigated }\end{array}$ & $\begin{array}{c}\text { Water } \\
\text { used } \\
\text { (acre-ft) }\end{array}$ & $\begin{array}{l}\text { Rate of } \\
\text { irriga- } \\
\text { tion } \\
\text { (acre- } \\
\text { ft/acre) }\end{array}$ & $\begin{array}{c}\text { Total } \\
\text { acres } \\
\text { irrigated }\end{array}$ & $\begin{array}{c}\text { Water } \\
\text { used } \\
\text { (acre-ft) }\end{array}$ & $\begin{array}{l}\text { Rate of } \\
\text { irriga- } \\
\text { tion } \\
\text { (acre- } \\
\text { ft/acre) }\end{array}$ & $\begin{array}{c}\text { Total } \\
\text { acres } \\
\text { irri- } \\
\text { gated }\end{array}$ & $\begin{array}{l}\text { Irriga- } \\
\text { tion } \\
\text { water } \\
\text { used } \\
\text { (acre- } \\
\text { ft) }\end{array}$ & $\begin{array}{l}\text { Rate of } \\
\text { irri- } \\
\text { gation } \\
\text { (acre- } \\
\text { ft/acre) }\end{array}$ \\
\hline 1991 & 19.84 & 262,528 & 355,589 & 1.35 & 216,196 & 299,120 & 1.38 & 34,172 & 39,671 & 1.16 & NA & NA & NA & 3,061 & 4,431 & 1.45 \\
\hline 1992 & 24.16 & 244,582 & 270,143 & 1.10 & 188,730 & 216,103 & 1.15 & 30,403 & 30,008 & .99 & 12,209 & 13,003 & 1.07 & 2,828 & 4,524 & 1.60 \\
\hline 1993 & 28.72 & 223,046 & 197,600 & .89 & 153,685 & 141,603 & .92 & 30,312 & 24,862 & .82 & 21,564 & 17,978 & .83 & 2,034 & 2,608 & 1.28 \\
\hline 1994 & 18.83 & 236,993 & 281,921 & 1.19 & 152,462 & 183,734 & 1.21 & 35,593 & 41,356 & 1.16 & 24,495 & 26,488 & 1.08 & 3,015 & 3,990 & 1.32 \\
\hline 1995 & 21.55 & 242,512 & 258,857 & 1.07 & 136,550 & 155,854 & 1.14 & 41,565 & 40,900 & .98 & 33,328 & 32,913 & .99 & 1,944 & 2,992 & 1.54 \\
\hline 1996 & 25.40 & 242,642 & 250,042 & 1.03 & 123,648 & 131,803 & 1.07 & 48,318 & 46,961 & .97 & 42,166 & 41,796 & .99 & 2,902 & 3,458 & 1.19 \\
\hline 1997 & 23.51 & 246,497 & 225,872 & .92 & 106,858 & 105,247 & .98 & 41,150 & 33,529 & .81 & 69,341 & 60,145 & .87 & 2,967 & 3,472 & 1.17 \\
\hline 1998 & 21.11 & 244,883 & 223,389 & .91 & 86,245 & 81,677 & .95 & 44,591 & 37,746 & .85 & 79,805 & 75,122 & .94 & 2,859 & 3,486 & 1.22 \\
\hline 1999 & 21.42 & 246,636 & 219,915 & .89 & 77,205 & 75,953 & .98 & 40,196 & 33,389 & .83 & 93,569 & 79,546 & .85 & 2,555 & 3,430 & 1.34 \\
\hline 2000 & 19.64 & 250,274 & 275,750 & 1.10 & 66,913 & 75,083 & 1.12 & 27,605 & 27,129 & .98 & 119,262 & 131,780 & 1.10 & 2,266 & 3,117 & 1.38 \\
\hline 2001 & 19.93 & 237,016 & 226,216 & .95 & 52,491 & 53,405 & 1.02 & 29,663 & 26,016 & .88 & 120,284 & 115,239 & .96 & 2,380 & 4,181 & 1.76 \\
\hline 2002 & 13.25 & 243,617 & 286,707 & 1.18 & 51,732 & 61,436 & 1.19 & 23,716 & 26,216 & 1.11 & 123,104 & 147,330 & 1.20 & 2,521 & 3,996 & 1.58 \\
\hline 2003 & 17.04 & 235,050 & 236,793 & 1.01 & 47,292 & 49,659 & 1.05 & 18,770 & 17,394 & .93 & 132,831 & 132,349 & 1.00 & 2,260 & 3,237 & 1.43 \\
\hline Average & 21.11 & 242,790 & 254,523 & 1.05 & 112,308 & 125,437 & 1.09 & 34,312 & 32,706 & .96 & 72,633 & 72,807 & .99 & 2,584 & 3,609 & 1.40 \\
\hline Mininum & 13.25 & 223,046 & 197,600 & .89 & 47,292 & 49,659 & .92 & 18,770 & 17,394 & .81 & 12,209 & 13,003 & .83 & 1,944 & 2,608 & 1.17 \\
\hline Maximum & 28.72 & 262,528 & 355,589 & 1.35 & 216,196 & 299,120 & 1.38 & 48,318 & 46,961 & 1.16 & 132,831 & 147,330 & 1.20 & 3,061 & 4,524 & 1.76 \\
\hline Median & 21.11 & 243,617 & 250,042 & 1.03 & 106,858 & 105,247 & 1.07 & 34,172 & 33,389 & .97 & 74,573 & 67,634 & .99 & 2,555 & 3,472 & 1.38 \\
\hline Slope & -.67 & -46 & $-3,252$ & -.015 & $-14,042$ & $-15,916$ & -.0106 & -790 & $-1,428$ & -.0055 & 12,004 & 12,158 & .004 & -50.7 & -34.8 & .012 \\
\hline p-value & .044 & 1.000 & .502 & .358 & 0 & 0 & .392 & .161 & .044 & .426 & 0 & 0 & .582 & .100 & .669 & .583 \\
\hline
\end{tabular}


Table 7. Trends in precipitation and irrigation by system type, crop type, and prorated crop type for Groundwater Management District 1 , west-central Kansas,

1991-2003.-Continued

[Data from Kansas Water Office and Kansas Department of Agriculture (1993-2005). acre-ft, acre-feet; acre-ft/acre, acre-foot per acre. Blue shading indicates trend is significant at 95-percent level ( $\mathrm{p} \leq 0.05)$. Green shading indicates trend is significant at 90 -percent level $(\mathrm{p} \leq 0.10)$. NA, data not available]

\begin{tabular}{|c|c|c|c|c|c|c|c|c|c|c|c|c|c|c|c|}
\hline \multirow[b]{2}{*}{ Year } & \multicolumn{3}{|c|}{ Corn } & \multicolumn{3}{|c|}{ Grain sorghum } & \multicolumn{3}{|c|}{ Soybeans } & \multicolumn{3}{|c|}{ Wheat } & \multicolumn{3}{|c|}{ Other/multiple use } \\
\hline & $\begin{array}{c}\text { Total } \\
\text { acres } \\
\text { irrigated }\end{array}$ & $\begin{array}{l}\text { Irrigation } \\
\text { water } \\
\text { used } \\
\text { (acre-ft) }\end{array}$ & $\begin{array}{c}\text { Rate of } \\
\text { irriga- } \\
\text { tion } \\
\text { (acre- } \\
\text { ft/acre) }\end{array}$ & $\begin{array}{c}\text { Total } \\
\text { acres } \\
\text { irrigated }\end{array}$ & $\begin{array}{l}\text { Irrigation } \\
\text { water } \\
\text { used } \\
\text { (acre-ft) }\end{array}$ & $\begin{array}{l}\text { Rate of } \\
\text { irriga- } \\
\text { tion } \\
\text { (acre- } \\
\text { ft/acre) }\end{array}$ & $\begin{array}{c}\text { Total } \\
\text { acres } \\
\text { irrigated }\end{array}$ & $\begin{array}{l}\text { Irrigation } \\
\text { water } \\
\text { used } \\
\text { (acre-ft) }\end{array}$ & $\begin{array}{c}\text { Rate of } \\
\text { irriga- } \\
\text { tion } \\
\text { (acre- } \\
\text { ft/acre) }\end{array}$ & $\begin{array}{c}\text { Total } \\
\text { acres } \\
\text { irrigated }\end{array}$ & $\begin{array}{l}\text { Irrigation } \\
\text { water } \\
\text { used } \\
\text { (acre-ft) }\end{array}$ & $\begin{array}{c}\text { Rate of } \\
\text { irriga- } \\
\text { tion } \\
\text { (acre- } \\
\text { ft/acre) }\end{array}$ & $\begin{array}{c}\text { Total } \\
\text { acres } \\
\text { irrigated }\end{array}$ & $\begin{array}{l}\text { Irrigation } \\
\text { water } \\
\text { used } \\
\text { (acre-ft) }\end{array}$ & $\begin{array}{c}\text { Rate of } \\
\text { irriga- } \\
\text { tion } \\
\text { (acre- } \\
\mathrm{ft} / \text { acre) }\end{array}$ \\
\hline 1991 & 41,584 & 64,116 & 1.54 & 15,576 & 18,257 & 1.17 & 301 & 431 & 1.43 & 7,655 & 5,800 & 0.76 & 194,351 & 262,554 & 1.35 \\
\hline 1992 & 48,455 & 62,838 & 1.30 & 12,940 & 11,602 & .90 & 916 & 1,003 & 1.10 & 7,974 & 5,652 & .71 & 171,469 & 184,524 & 1.08 \\
\hline 1993 & 58,762 & 64,761 & 1.10 & 9,997 & 7,270 & .73 & 67 & 17 & .26 & 5,459 & 2,409 & .44 & 146,727 & 120,534 & .82 \\
\hline 1994 & 50,703 & 73,305 & 1.45 & 10,029 & 8,526 & .85 & 317 & 403 & 1.27 & 4,566 & 2,477 & .54 & 168,363 & 193,220 & 1.15 \\
\hline 1995 & 54,699 & 68,045 & 1.24 & 9,488 & 8,107 & .85 & 0 & 0 & 0 & 7,371 & 5,126 & .70 & 169,010 & 174,586 & 1.03 \\
\hline 1996 & 66,332 & 82,493 & 1.24 & 9,267 & 6,801 & .73 & 118 & 82 & .69 & 4,597 & 2,082 & .45 & 159,426 & 155,127 & .97 \\
\hline 1997 & 69,309 & 76,172 & 1.10 & 7,183 & 4,226 & .59 & 544 & 416 & .76 & 9,338 & 5,386 & .58 & 157,156 & 136,200 & .87 \\
\hline 1998 & 69,902 & 79,262 & 1.13 & 2,960 & 1,659 & .56 & 1,362 & 1,027 & .75 & 6,647 & 4,429 & .67 & 161,153 & 133,526 & .83 \\
\hline 1999 & 68,483 & 74,841 & 1.09 & 3,181 & 2,672 & .84 & 1,462 & 1,612 & 1.10 & 5,492 & 2,454 & .45 & 165,463 & 134,906 & .82 \\
\hline 2000 & 68,448 & 94,918 & 1.39 & 2,494 & 2,221 & .89 & 1,600 & 1,499 & .94 & 6,651 & 4,168 & .63 & 168,815 & 169,827 & 1.01 \\
\hline 2001 & 54,499 & 66,954 & 1.23 & 5,128 & 3,636 & .71 & 1,189 & 1,203 & 1.01 & 5,351 & 2,524 & .47 & 168,469 & 147,718 & .88 \\
\hline 2002 & 47,875 & 75,844 & 1.58 & 4,553 & 5,068 & 1.11 & 1,520 & 1,550 & 1.02 & 5,613 & 4,289 & .76 & 181,535 & 195,960 & 1.08 \\
\hline 2003 & 31,591 & 45,476 & 1.44 & 5,649 & 4,257 & .75 & 839 & 778 & .93 & 10,641 & 5,751 & .54 & 184,070 & 177,294 & .96 \\
\hline Average & 56,203 & 71,463 & 1.29 & 7,573 & 6,485 & .82 & 787 & 771 & .87 & 6,720 & 4,042 & .59 & 168,924 & 168,152 & .99 \\
\hline Mininum & 31,591 & 45,476 & 1.09 & 2,494 & 1,659 & .71 & 0 & 0 & 0 & 4,566 & 2,082 & .44 & 146,727 & 120,534 & .82 \\
\hline Maximum & 69,902 & 94,918 & 1.58 & 15,576 & 18,257 & 1.17 & 1,600 & 1,612 & 1.43 & 10,641 & 5,800 & .76 & 194,351 & 262,554 & 1.35 \\
\hline Median & 54,699 & 73,305 & 1.24 & 7,183 & 5,068 & .84 & 839 & 778 & .94 & 6,647 & 4,289 & .58 & 168,469 & 169,827 & .97 \\
\hline Slope & 582 & 1,024 & -.0005 & -833 & -771 & -.0098 & 103 & 92.1 & -.0016 & 3.75 & -19.8 & -.0067 & 1,324 & $-1,625$ & -.0173 \\
\hline $\mathrm{p}$-value & .760 & .300 & .951 & .002 & .009 & .358 & .033 & .059 & 1.000 & .951 & .855 & .713 & .502 & .669 & .245 \\
\hline
\end{tabular}


Table 7. Trends in precipitation and irrigation by system type, crop type, and prorated crop type for Groundwater Management District 1, west-central Kansas, 1991-2003.-Continued

[Data from Kansas Water Office and Kansas Department of Agriculture (1993-2005). acre-ft, acre-feet; acre-ft/acre, acre-foot per acre. Blue shading indicates trend is significant at 95-percent level $(\mathrm{p} \leq 0.05)$. Green shading indicates trend is significant at 90 -percent level ( $\mathrm{p} \leq 0.10)$. NA, data not available]

\begin{tabular}{|c|c|c|c|c|c|c|}
\hline \multirow[b]{2}{*}{ Year } & \multicolumn{6}{|c|}{ Total acres irrigated } \\
\hline & $\begin{array}{l}\text { Prorated } \\
\text { alfalfa }\end{array}$ & Prorated corn & $\begin{array}{l}\text { Prorated grain } \\
\text { sorghum }\end{array}$ & $\begin{array}{l}\text { Prorated } \\
\text { soybeans }\end{array}$ & $\begin{array}{c}\text { Prorated } \\
\text { wheat }\end{array}$ & $\begin{array}{c}\text { Other } \\
\text { prorated, } \\
\text { unspecified }\end{array}$ \\
\hline 1991 & 18,862 & 120,982 & 44,807 & 301 & 55,452 & 22,124 \\
\hline 1992 & 10,258 & 101,980 & 41,191 & 7,218 & 66,767 & 17,168 \\
\hline 1993 & 7,869 & 111,027 & 28,153 & 2,284 & 59,130 & 14,583 \\
\hline 1994 & 9,632 & 110,004 & 29,460 & 3,729 & 68,482 & 15,687 \\
\hline 1995 & 9,116 & 115,964 & 27,816 & 1,408 & 69,982 & 18,226 \\
\hline 1996 & 8,707 & 123,898 & 31,552 & 3,462 & 57,281 & 17,742 \\
\hline 1997 & 7,291 & 132,274 & 24,597 & 5,782 & 67,264 & 9,288 \\
\hline 1998 & 11,128 & 128,234 & 14,695 & 13,215 & 59,377 & 18,234 \\
\hline 1999 & 7,485 & 131,867 & 14,839 & 10,560 & 62,341 & 19,544 \\
\hline 2000 & 6,086 & 133,715 & 9,369 & 12,099 & 70,411 & 18,594 \\
\hline 2001 & 5,491 & 115,679 & 17,576 & 11,217 & 66,808 & 20,186 \\
\hline 2002 & 5,183 & 115,246 & 23,284 & 8,449 & 76,414 & 15,041 \\
\hline 2003 & 5,719 & 89,352 & 22,681 & 9,930 & 78,208 & 29,161 \\
\hline Average & 8,679 & 117,709 & 25,386 & 6,896 & 65,994 & 18,121 \\
\hline Mininum & 5,183 & 89,352 & 9,369 & 301 & 55,452 & 9,288 \\
\hline Maximum & 18,862 & 133,715 & 44,807 & 13,215 & 78,208 & 29,161 \\
\hline Median & 7,869 & 115,964 & 24,597 & 7,218 & 66,808 & 18,226 \\
\hline Slope & -485 & 732 & $-1,900$ & 852 & 1,076 & 328 \\
\hline $\mathrm{p}$-value & .001 & .128 & .006 & .024 & .0173 & .2001 \\
\hline
\end{tabular}


Table 8. Trends in precipitation and irrigation by system type, crop type, and prorated crop type for Ground Water Management District 2, south-central Kansas, 1991-2003.

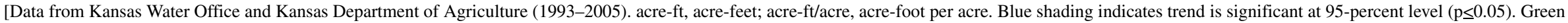
shading indicates trend is significant at 90 -percent level $(\mathrm{p} \leq 0.10)$. NA, data not available]

\begin{tabular}{|c|c|c|c|c|c|c|c|c|c|c|c|c|c|c|c|c|}
\hline \multirow[b]{2}{*}{ Year } & \multirow{2}{*}{$\begin{array}{l}\text { Meteor- } \\
\text { ological } \\
\text { region } 8 \\
\text { precipi- } \\
\text { tation } \\
\text { (inches) }\end{array}$} & \multirow[b]{2}{*}{$\begin{array}{c}\text { Total } \\
\text { acres } \\
\text { irrigated }\end{array}$} & \multirow[b]{2}{*}{$\begin{array}{c}\text { Total } \\
\text { irrigation } \\
\text { water use } \\
\text { (acre-ft) }\end{array}$} & \multirow{2}{*}{$\begin{array}{c}\text { Average } \\
\text { rate of } \\
\text { irrigation } \\
\text { (acre- } \\
\text { ft/acre) }\end{array}$} & \multicolumn{3}{|c|}{ Flood irrigation } & \multicolumn{3}{|c|}{ Center pivot irrigation } & \multicolumn{3}{|c|}{$\begin{array}{l}\text { Center pivot irrigation with drop } \\
\text { nozzle }\end{array}$} & \multicolumn{3}{|c|}{ Alfalfa } \\
\hline & & & & & $\begin{array}{c}\text { Total } \\
\text { acres } \\
\text { irrigated }\end{array}$ & $\begin{array}{c}\text { Water } \\
\text { used } \\
\text { (acre-ft) }\end{array}$ & $\begin{array}{c}\text { Rate of } \\
\text { irrigation } \\
\text { (acre- } \\
\text { ft/acre) }\end{array}$ & $\begin{array}{c}\text { Total } \\
\text { acres } \\
\text { irrigated }\end{array}$ & $\begin{array}{c}\text { Water } \\
\text { used } \\
\text { (acre-ft) }\end{array}$ & $\begin{array}{c}\text { Rate of } \\
\text { irriga- } \\
\text { tion } \\
\text { (acre- } \\
\mathrm{ft} / \text { acre) }\end{array}$ & $\begin{array}{c}\text { Total } \\
\text { acres } \\
\text { irrigated }\end{array}$ & $\begin{array}{c}\text { Water } \\
\text { used } \\
\text { (acre-ft) }\end{array}$ & $\begin{array}{c}\text { Rate of } \\
\text { irriga- } \\
\text { tion } \\
\text { (acre- } \\
\text { ft/acre) }\end{array}$ & $\begin{array}{l}\text { Total } \\
\text { acres irri- } \\
\text { gated }\end{array}$ & $\begin{array}{c}\text { Irrigation } \\
\text { waterused } \\
\text { (acre-ft) }\end{array}$ & $\begin{array}{c}\text { Rate of } \\
\text { irri- } \\
\text { gation } \\
\text { (acre- } \\
\mathrm{ft} / \text { acre) }\end{array}$ \\
\hline 1991 & 22.72 & 97,833 & 112,604 & 1.15 & 37,604 & 47,438 & 1.26 & 52,475 & 57,681 & 1.10 & NA & NA & NA & 1,311 & 1,173 & 0.90 \\
\hline 1992 & 31.96 & 92,880 & 57,195 & .62 & 33,125 & 22,523 & .68 & 52,029 & 30,556 & .59 & 1,608 & 619 & .38 & 1,730 & 1,077 & .62 \\
\hline 1993 & 34.08 & 90,525 & 59,150 & .65 & 31,261 & 22,283 & .71 & 52,474 & 33,068 & .63 & 1,048 & 711 & .68 & 1,000 & 507 & .51 \\
\hline 1994 & 22.06 & 97,243 & 100,583 & 1.03 & 33,648 & 38,253 & 1.14 & 53,525 & 52,899 & .99 & 1,108 & 872 & .79 & 1,179 & 1,382 & 1.17 \\
\hline 1995 & 31.66 & 97,814 & 84,940 & .87 & 31,246 & 29,015 & .93 & 56,895 & 48,279 & .85 & 1,669 & 1,094 & .66 & 1,178 & 717 & .61 \\
\hline 1996 & 32.01 & 103,597 & 86,645 & .84 & 29,702 & 28,537 & .96 & 63,406 & 50,110 & .79 & 2,289 & 1,924 & .84 & 1,195 & 847 & .71 \\
\hline 1997 & 34.39 & 106,771 & 76,457 & .72 & 27,709 & 22,793 & .82 & 42,920 & 29,687 & .69 & 28,158 & 18,578 & .66 & 1,376 & 887 & .64 \\
\hline 1998 & 30.14 & 110,441 & 100,825 & .91 & 26,502 & 27,643 & 1.04 & 39,608 & 32,975 & .83 & 32,294 & 29,975 & .93 & 1,656 & 1,527 & .92 \\
\hline 1999 & 32.17 & 114,635 & 85,121 & .74 & 26,588 & 22,574 & .85 & 35,230 & 24,622 & .70 & 42,404 & 30,645 & .72 & 1,248 & 883 & .71 \\
\hline 2000 & 32.76 & 116,186 & 112,448 & .97 & 26,875 & 30,136 & 1.12 & 25,159 & 21,843 & .87 & 52,818 & 50,443 & .96 & 918 & 934 & 1.02 \\
\hline 2001 & 24.87 & 117,476 & 131,639 & 1.12 & 25,419 & 31,743 & 1.25 & 25,522 & 26,152 & 1.02 & 54,087 & 60,227 & 1.11 & 1,365 & 1,089 & .80 \\
\hline 2002 & 29.41 & 120,840 & 125,933 & 1.04 & 24,826 & 30,690 & 1.24 & 20,090 & 20,116 & 1.00 & 61,401 & 61,132 & 1.00 & 1,495 & 1,321 & .88 \\
\hline 2003 & 28.41 & 121,404 & 138,811 & 1.14 & 22,520 & 29,566 & 1.31 & 19,362 & 21,060 & 1.09 & 63,854 & 70,842 & 1.11 & 1,363 & 930 & .68 \\
\hline Average & 29.74 & 106,742 & 97,873 & .91 & 29,002 & 29,476 & 1.02 & 41,438 & 34,542 & .86 & 28,562 & 27,255 & .82 & 1,309 & 1,021 & .78 \\
\hline Mininum & 22.06 & 90,525 & 57,195 & .62 & 22,520 & 22,283 & .68 & 19,362 & 20,116 & .59 & 1,048 & 619 & .38 & 918 & 507 & .51 \\
\hline Maximum & 34.39 & 121,404 & 138,811 & 1.15 & 37,604 & 47,438 & 1.31 & 63,406 & 57,681 & 1.10 & 63,854 & 70,842 & 1.11 & 1,730 & 1,527 & 1.17 \\
\hline Median & 31.66 & 106,771 & 100,583 & .91 & 27,709 & 29,015 & 1.04 & 42,920 & 30,556 & .85 & 30,226 & 24,277 & .82 & 1,311 & 934 & .71 \\
\hline Slope & -.14 & 2,843 & 6,066 & .0312 & $-1,020$ & 98.2 & .04 & $-3,363$ & $-2,457$ & .0248 & 6,677 & 7,143 & .05 & 15.3 & 14.6 & .013 \\
\hline p-value & .951 & 0 & .012 & .100 & 0 & .669 & .059 & .003 & .002 & .127 & 0 & 0 & .002 & .583 & .502 & .463 \\
\hline
\end{tabular}


Table 8. Trends in precipitation and irrigation by system type, crop type, and prorated crop type for Ground Water Management District 2, south-central Kansas, 19912003.-Continued

[Data from Kansas Water Office and Kansas Department of Agriculture (1993-2005). acre-ft, acre-feet; acre-ft/acre, acre-foot per acre. Blue shading indicates trend is significant at 95-percent level ( $\mathrm{p} \leq 0.05$ ). Green shading indicates trend is significant at 90 -percent level $(\mathrm{p} \leq 0.10)$. NA, data not available]

\begin{tabular}{|c|c|c|c|c|c|c|c|c|c|c|c|c|c|c|c|}
\hline \multirow[b]{2}{*}{ Year } & \multicolumn{3}{|c|}{ Corn } & \multicolumn{3}{|c|}{ Grain sorghum } & \multicolumn{3}{|c|}{ Soybeans } & \multicolumn{3}{|c|}{ Wheat } & \multicolumn{3}{|c|}{ Other/multiple use } \\
\hline & $\begin{array}{c}\text { Total } \\
\text { acres } \\
\text { irrigated }\end{array}$ & $\begin{array}{l}\text { Irrigation } \\
\text { water } \\
\text { used } \\
\text { (acre-ft) }\end{array}$ & $\begin{array}{c}\text { Rate of } \\
\text { irriga- } \\
\text { tion } \\
\text { (acre- } \\
\text { ft/acre) }\end{array}$ & $\begin{array}{c}\text { Total } \\
\text { acres } \\
\text { irrigated }\end{array}$ & $\begin{array}{l}\text { Irrigation } \\
\text { water } \\
\text { used } \\
\text { (acre-ft) }\end{array}$ & $\begin{array}{l}\text { Rate of } \\
\text { irrigation } \\
\text { (acre- } \\
\text { ft/acre) }\end{array}$ & $\begin{array}{c}\text { Total } \\
\text { acres } \\
\text { irrigated }\end{array}$ & $\begin{array}{c}\text { Irrigation } \\
\text { water } \\
\text { used } \\
\text { (acre-ft) }\end{array}$ & $\begin{array}{c}\text { Rate of } \\
\text { irrigation } \\
\text { (acre- } \\
\text { ft/acre) }\end{array}$ & $\begin{array}{c}\text { Total acres } \\
\text { irrigated }\end{array}$ & $\begin{array}{l}\text { Irrigation } \\
\text { water } \\
\text { used } \\
\text { (acre-ft) }\end{array}$ & $\begin{array}{c}\text { Rate of } \\
\text { irrigation } \\
\text { (acre- } \\
\text { ft/acre) }\end{array}$ & $\begin{array}{c}\text { Total } \\
\text { acres } \\
\text { irrigated }\end{array}$ & $\begin{array}{l}\text { Irrigation } \\
\text { water } \\
\text { used } \\
\text { (acre-ft) }\end{array}$ & $\begin{array}{c}\text { Rate of } \\
\text { irriga- } \\
\text { tion } \\
\text { (acre- } \\
\text { ft/acre) }\end{array}$ \\
\hline 1991 & 22,721 & 29,650 & 1.30 & 7,304 & 5,589 & 0.77 & 13,733 & 15,487 & 1.13 & 721 & 418 & 0.58 & 52,043 & 60,286 & 1.16 \\
\hline 1992 & 19,827 & 11,723 & .59 & 8,719 & 4,837 & .55 & 13,589 & 9,599 & .71 & 927 & 329 & .36 & 48,088 & 29,629 & .62 \\
\hline 1993 & 20,270 & 14,016 & .69 & 4,813 & 2,213 & .46 & 12,330 & 9,371 & .76 & 633 & 114 & .18 & 51,479 & 32,928 & .64 \\
\hline 1994 & 21,342 & 24,224 & 1.14 & 4,076 & 3,498 & .86 & 16,155 & 17,032 & 1.05 & 802 & 213 & .27 & 53,689 & 54,234 & 1.01 \\
\hline 1995 & 20,084 & 19,902 & .99 & 7,060 & 4,822 & .68 & 15,803 & 14,032 & .89 & 1,227 & 393 & .32 & 52,462 & 45,075 & .86 \\
\hline 1996 & 24,474 & 24,680 & 1.01 & 5,578 & 3,615 & .65 & 15,063 & 11,988 & .80 & 1,490 & 489 & .33 & 55,797 & 45,027 & .81 \\
\hline 1997 & 26,924 & 21,082 & .78 & 3,876 & 2,174 & .56 & 21,919 & 15,898 & .73 & 1,401 & 424 & .30 & 51,275 & 35,993 & .70 \\
\hline 1998 & 27,370 & 25,714 & .94 & 3,698 & 2,576 & .70 & 24,134 & 23,689 & .98 & 1,012 & 476 & .47 & 52,571 & 46,844 & .89 \\
\hline 1999 & 30,616 & 22,628 & .74 & 2,993 & 1,768 & .59 & 29,126 & 22,773 & .78 & 684 & 239 & .35 & 49,968 & 36,830 & .74 \\
\hline 2000 & 28,112 & 25,284 & .90 & 2,816 & 1,929 & .69 & 33,760 & 36,285 & 1.07 & 729 & 250 & .34 & 49,851 & 47,766 & .96 \\
\hline 2001 & 30,904 & 34,676 & 1.12 & 3,134 & 2,722 & .87 & 31,783 & 36,951 & 1.16 & 1,194 & 663 & .55 & 49,096 & 55,537 & 1.13 \\
\hline 2002 & 35,974 & 38,735 & 1.08 & 3,356 & 2,523 & .75 & 29,202 & 31,773 & 1.09 & 635 & 408 & .64 & 50,178 & 51,173 & 1.02 \\
\hline 2003 & 43,078 & 50,765 & 1.18 & 4,352 & 3,659 & .84 & 24,628 & 29,815 & 1.21 & 594 & 251 & .42 & 47,389 & 53,390 & 1.13 \\
\hline Average & 27,054 & 26,391 & .96 & 4,752 & 3,225 & .69 & 21,633 & 21,130 & .95 & 927 & 359 & .39 & 51,068 & 45,747 & .90 \\
\hline Mininum & 19,827 & 11,723 & .59 & 2,816 & 1,768 & .46 & 12,330 & 9,371 & .71 & 594 & 114 & .18 & 47,389 & 29,629 & .62 \\
\hline Maximum & 43,078 & 50,765 & 1.30 & 8,719 & 5,589 & .87 & 33,760 & 36,951 & 1.21 & 1,490 & 663 & .64 & 55,797 & 60,286 & 1.16 \\
\hline Median & 26,924 & 24,680 & .99 & 4,076 & 2,722 & .69 & 21,919 & 17,032 & .98 & 802 & 393 & .35 & 51,275 & 46,844 & .89 \\
\hline Slope & 1,524 & 2,146 & .0218 & -320 & -186 & .017 & 1,747 & 2,095 & .0292 & -14.2 & 5.68 & .0163 & -296 & 1,026 & .0312 \\
\hline $\mathrm{p}$-value & 0 & .006 & .428 & .009 & .127 & .161 & .002 & .004 & .410 & .502 & .502 & .127 & .100 & .282 & .112 \\
\hline
\end{tabular}


Table 8. Trends in precipitation and irrigation by system type, crop type, and prorated crop type for Ground Water Management District 2, south-central Kansas, 1991-2003.-Continued

[Data from Kansas Water Office and Kansas Department of Agriculture (1993-2005). acre-ft, acre-feet; acre-ft/acre, acre-foot per acre. Blue shading indicates trend is significant at 95-percent level $(\mathrm{p} \leq 0.05)$. Green shading indicates trend is significant at 90 -percent level $(\mathrm{p} \leq 0.10)$. NA, data not available]

\begin{tabular}{crrrrrr}
\hline & \multicolumn{5}{c}{ Total acres irrigated } \\
\cline { 2 - 7 } Year & $\begin{array}{c}\text { Prorated } \\
\text { alfalfa }\end{array}$ & $\begin{array}{c}\text { Prorated } \\
\text { corn }\end{array}$ & $\begin{array}{c}\text { Prorated } \\
\text { grain } \\
\text { sorghum }\end{array}$ & $\begin{array}{c}\text { Prorated } \\
\text { soybeans }\end{array}$ & $\begin{array}{c}\text { Prorated } \\
\text { wheat }\end{array}$ & $\begin{array}{c}\text { Other } \\
\text { prorated, } \\
\text { unspecified }\end{array}$ \\
\hline 1991 & 1,311 & 22,721 & 7,304 & 13,733 & 721 & 1,438 \\
1992 & 3,306 & 34,526 & 16,888 & 32,106 & 3,715 & 2,315 \\
1993 & 3,304 & 38,062 & 11,240 & 33,490 & 2,132 & 2,296 \\
1994 & 2,885 & 40,638 & 10,468 & 37,519 & 2,845 & 2,888 \\
1995 & 2,841 & 37,541 & 14,458 & 36,728 & 4,371 & 1,875 \\
& & & & & & \\
1996 & 3,373 & 43,140 & 13,671 & 35,182 & 6,392 & 1,839 \\
1997 & 3,091 & 45,876 & 8,375 & 42,487 & 5,060 & 1,881 \\
1998 & 3,124 & 46,780 & 7,409 & 45,637 & 3,462 & 4,028 \\
1999 & 3,059 & 47,669 & 6,674 & 48,955 & 3,399 & 4,878 \\
2000 & 2,067 & 46,654 & 6,462 & 54,219 & 2,678 & 4,107 \\
& & & & & & \\
2001 & 2,415 & 47,952 & 6,453 & 51,239 & 3,951 & 5,466 \\
2002 & 2,819 & 52,636 & 7,716 & 48,063 & 4,757 & 4,849 \\
2003 & 2,757 & 59,169 & 8,441 & 42,448 & 3,139 & 5,450 \\
Average & 2,796 & 43,336 & 9,658 & 40,139 & 3,586 & 3,332 \\
Mininum & 1,311 & 22,721 & 6,453 & 13,733 & 721 & 1,438 \\
Maximum & 3,373 & 59,169 & 16,888 & 54,219 & 6,392 & 5,466 \\
Median & 2,885 & 45,876 & 8,375 & 42,448 & 3,462 & 2,888 \\
Slope & -43.5 & 1,850 & -618 & 2233 & 132 & 324 \\
p-value & .200 & 0 & .059 & .002 & .428 & .004 \\
\hline
\end{tabular}


Table 9. Trends in precipitation and irrigation by system type, crop type, and prorated crop type for Groundwater Management District 3, southwest Kansas, $1991-2003$.

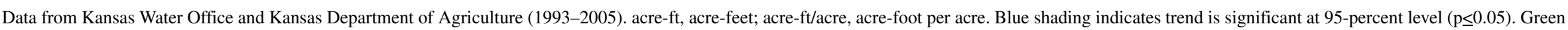
shading indicates trend is significant at 90 -percent level $(\mathrm{p} \leq 0.10)$. NA, data not available]

\begin{tabular}{|c|c|c|c|c|c|c|c|c|c|c|c|c|c|c|c|c|}
\hline \multirow[b]{2}{*}{ Year } & \multirow{2}{*}{$\begin{array}{l}\text { Meteor- } \\
\text { ological } \\
\text { region } 7 \\
\text { precipi- } \\
\text { tation } \\
\text { (inches) }\end{array}$} & \multirow[b]{2}{*}{$\begin{array}{c}\text { Total acres } \\
\text { irrigated }\end{array}$} & \multirow[b]{2}{*}{$\begin{array}{c}\text { Total } \\
\text { irrigation } \\
\text { water use } \\
\text { (acre-ft) }\end{array}$} & \multirow{2}{*}{$\begin{array}{c}\text { Aver- } \\
\text { age rate } \\
\text { of irriga- } \\
\text { tion } \\
\text { (acre- } \\
\text { ft/acre) }\end{array}$} & \multicolumn{3}{|c|}{ Flood irrigation } & \multicolumn{3}{|c|}{ Center pivot irrigation } & \multicolumn{3}{|c|}{$\begin{array}{l}\text { Center pivot irrigation with } \\
\text { drop nozzle }\end{array}$} & \multicolumn{3}{|c|}{ Alfalfa } \\
\hline & & & & & $\begin{array}{c}\text { Total } \\
\text { acres } \\
\text { irrigated }\end{array}$ & $\begin{array}{c}\text { Water } \\
\text { used } \\
\text { (acre-ft) }\end{array}$ & $\begin{array}{c}\text { Rate of } \\
\text { irriga- } \\
\text { tion } \\
\text { (acre- } \\
\text { ft/acre) }\end{array}$ & $\begin{array}{c}\text { Total } \\
\text { acres } \\
\text { irrigated }\end{array}$ & $\begin{array}{c}\text { Water } \\
\text { used } \\
\text { (acre-ft) }\end{array}$ & $\begin{array}{c}\text { Rate of } \\
\text { irriga- } \\
\text { tion } \\
\text { (acre- } \\
\mathrm{ft} / \text { acre) }\end{array}$ & $\begin{array}{c}\text { Total } \\
\text { acres } \\
\text { irrigated }\end{array}$ & $\begin{array}{c}\text { Water } \\
\text { used } \\
\text { (acre-ft) }\end{array}$ & $\begin{array}{c}\text { Rate of } \\
\text { irriga- } \\
\text { tion } \\
\text { (acre- } \\
\text { ft/acre) }\end{array}$ & $\begin{array}{c}\text { Total } \\
\text { acres } \\
\text { irri- } \\
\text { gated }\end{array}$ & $\begin{array}{l}\text { Irrigation } \\
\text { waterused } \\
\text { (acre-ft) }\end{array}$ & $\begin{array}{c}\text { Rate of } \\
\text { irri- } \\
\text { gation } \\
\text { (acre- } \\
\mathrm{ft} / \text { acre) }\end{array}$ \\
\hline 1991 & 18.19 & $1,593,191$ & $2,660,233$ & 1.67 & 823,757 & $1,417,562$ & 1.72 & 604,248 & 974,993 & 1.61 & NA & NA & NA & 112,077 & 227,401 & 2.03 \\
\hline 1992 & 19.69 & $1,580,084$ & $2,178,273$ & 1.38 & 783,385 & $1,120,560$ & 1.43 & 574,112 & 773,720 & 1.35 & 52,305 & 62,683 & 1.20 & 104,396 & 175,814 & 1.68 \\
\hline 1993 & 24.11 & $1,550,396$ & $1,887,823$ & 1.22 & 688,521 & 859,444 & 1.25 & 601,448 & 740,184 & 1.23 & 56,285 & 62,973 & 1.12 & 103,698 & 62,041 & 1.56 \\
\hline 1994 & 18.87 & $1,555,565$ & $2,239,152$ & 1.44 & 602,004 & 903,099 & 1.50 & 609,548 & 868,895 & 1.43 & 88,960 & 124,034 & 1.39 & 109,133 & 199,017 & 1.82 \\
\hline 1995 & 18.45 & $1,533,409$ & $2,109,913$ & 1.38 & 486,039 & 708,401 & 1.46 & 657,248 & 882,965 & 1.34 & 93,271 & 125,052 & 1.34 & 114,411 & 193,784 & 1.69 \\
\hline 1996 & 25.58 & $1,524,257$ & $1,949,992$ & 1.28 & 442,764 & 597,295 & 1.35 & 684,371 & 857,864 & 1.25 & 113,215 & 141,655 & 1.25 & 108,819 & 168,783 & 1.55 \\
\hline 1997 & 23.68 & $1,576,430$ & $1,851,794$ & 1.17 & 355,973 & 431,904 & 1.21 & 516,487 & 623,105 & 1.21 & 393,274 & 465,788 & 1.18 & 124,470 & 202,750 & 1.63 \\
\hline 1998 & 20.89 & $1,583,403$ & $1,940,161$ & 1.23 & 280,187 & 326,765 & 1.17 & 481,008 & 619,532 & 1.29 & 474,580 & 599,184 & 1.26 & 136,561 & 233,416 & 1.71 \\
\hline 1999 & 20.36 & $1,594,865$ & $1,759,432$ & & 239,048 & 259,471 & & & & 1.14 & 586,912 & & 1.13 & 130,1 & 185,079 & 1.42 \\
\hline 2000 & 18.82 & $1,584,541$ & $2,117,282$ & 1.34 & 207,349 & 265,080 & 1.28 & 327,109 & 444,594 & 1.36 & 716,366 & 995,219 & 1.39 & 122,033 & 222,914 & 1.83 \\
\hline 2001 & 18.45 & $1,552,436$ & $1,889,226$ & 1.22 & 163,657 & 187,146 & & 277,253 & 338,657 & 1.22 & 771,727 & 974,471 & 1.26 & 144,614 & 240,336 & 1.66 \\
\hline 2002 & 13.43 & $1,594,975$ & $2,357,519$ & 1.48 & 150,509 & 234,006 & 1.55 & 238,835 & 347,155 & 1.45 & 818,106 & $1,238,698$ & 1.51 & 159,240 & 298,900 & 1.88 \\
\hline 2003 & 17.98 & $1,514,599$ & $1,937,199$ & 1.28 & 112,449 & 144,707 & 1.29 & 215,483 & 266,573 & 1.24 & 842,524 & $1,094,106$ & 1.30 & 156,530 & 267,136 & 1.71 \\
\hline Average & 19.88 & $1,564,473$ & $2,067,538$ & 1.32 & 410,434 & 573,495 & 1.34 & 476,674 & 631,306 & 1.32 & 417,294 & 545,641 & 1.28 & 125,090 & 213,644 & 1.71 \\
\hline Mininum & 13.43 & $1,514,599$ & $1,759,432$ & 1.10 & 112,449 & 144,707 & 1.09 & 215,483 & 266,573 & 1.14 & 52,305 & 62,683 & 1.12 & 103,698 & 162,041 & 1.42 \\
\hline Maximum & 25.58 & $1,594,975$ & $2,660,233$ & 1.67 & 823,757 & $1,417,562$ & 1.72 & 684,371 & 974,993 & 1.61 & 842,524 & $1,238,698$ & 1.51 & 159,240 & 298,900 & 2.03 \\
\hline Median & 18.87 & $1,576,430$ & $1,949,992$ & 1.28 & 355,973 & 431,904 & 1.29 & 516,487 & 623,105 & 1.29 & 433,927 & 532,486 & 1.26 & 122,033 & 202,750 & 1.69 \\
\hline Slope & -.39 & 99.4 & $-21,752$ & -.017 & $-62,954$ & $-94,647$ & -.025 & $-38,507$ & $-58,984$ & -.012 & 84,806 & 117,084 & .01 & 4,884 & 7,368 & .002 \\
\hline p-value & .142 & .026 & .246 & .269 & 0 & 0 & .127 & .002 & 0 & .300 & 0 & 0 & .241 & .001 & .017 & .903 \\
\hline
\end{tabular}


Table 9. Trends in precipitation and irrigation by system type, crop type, and prorated crop type for Groundwater Management District 3, southwest Kansas, 1991-

Data from Kansas Water Office and Kansas Department of Agriculture (1993-2005). acre-ft, acre-feet; acre-ft/acre, acre-foot per acre. Blue shading indicates trend is significant at 95-percent level (p $\leq 0.05$ ). Green shading indicates trend is significant at 90 -percent level $(\mathrm{p} \leq 0.10)$. NA, data not available

\begin{tabular}{|c|c|c|c|c|c|c|c|c|c|c|c|c|c|c|c|}
\hline \multirow[b]{2}{*}{ Year } & \multicolumn{3}{|c|}{ Corn } & \multicolumn{3}{|c|}{ Grain sorghum } & \multicolumn{3}{|c|}{ Soybeans } & \multicolumn{3}{|c|}{ Wheat } & \multicolumn{3}{|c|}{ Other/multiple use } \\
\hline & $\begin{array}{c}\text { Total } \\
\text { acres } \\
\text { irrigated }\end{array}$ & $\begin{array}{l}\text { Irrigation } \\
\text { water } \\
\text { used } \\
\text { (acre-ft) }\end{array}$ & $\begin{array}{c}\text { Rate of } \\
\text { irriga- } \\
\text { tion } \\
\text { (acre- } \\
\text { ft/acre) }\end{array}$ & $\begin{array}{c}\text { Total } \\
\text { acres } \\
\text { irrigated }\end{array}$ & $\begin{array}{l}\text { Irrigation } \\
\text { water } \\
\text { used } \\
\text { (acre-ft) }\end{array}$ & $\begin{array}{c}\text { Rate of } \\
\text { irriga- } \\
\text { tion } \\
\text { (acre- } \\
\text { ft/acre) }\end{array}$ & $\begin{array}{c}\text { Total } \\
\text { acres } \\
\text { irrigated }\end{array}$ & $\begin{array}{l}\text { Irrigation } \\
\text { water } \\
\text { used } \\
\text { (acre-ft) }\end{array}$ & $\begin{array}{l}\text { Rate of } \\
\text { irriga- } \\
\text { tion } \\
\text { (acre- } \\
\text { ft/acre) }\end{array}$ & $\begin{array}{c}\text { Total acres } \\
\text { irrigated }\end{array}$ & $\begin{array}{c}\text { Irrigation } \\
\text { water } \\
\text { used } \\
\text { (acre-ft) }\end{array}$ & $\begin{array}{c}\text { Rate of } \\
\text { irriga- } \\
\text { tion } \\
\text { (acre- } \\
\text { ft/acre) }\end{array}$ & $\begin{array}{l}\text { Total acres } \\
\text { irrigated }\end{array}$ & $\begin{array}{l}\text { Irrigation } \\
\text { water } \\
\text { used } \\
\text { (acre-ft) }\end{array}$ & $\begin{array}{l}\text { Rate of } \\
\text { irriga- } \\
\text { tion } \\
\text { (acre- } \\
\text { ft/acre) }\end{array}$ \\
\hline 1991 & 186,557 & 356,541 & 1.91 & 49,141 & 72,187 & 1.47 & 9,440 & 14,308 & 1.52 & 82,560 & 90,641 & 1.10 & $1,153,416$ & $1,899,155$ & 1.65 \\
\hline 1992 & 217,375 & 336,529 & 1.55 & 42,784 & 39,958 & .93 & 10,676 & 10,342 & .97 & 79,339 & 81,315 & 1.02 & $1,125,514$ & $1,534,314$ & 1.36 \\
\hline 1993 & 259,940 & 390,866 & 1.50 & 26,218 & 21,496 & .82 & 6,799 & 6,715 & .99 & 68,829 & 55,768 & .81 & $1,084,912$ & $1,250,936$ & 1.15 \\
\hline 1994 & 277,076 & 464,174 & 1.68 & 20,143 & 20,990 & 1.04 & 6,735 & 9,086 & 1.35 & 59,655 & 56,857 & .95 & $1,082,823$ & $1,489,030$ & 1.38 \\
\hline 1995 & 278,912 & 450,640 & 1.62 & 20,033 & 19,878 & .99 & 4,709 & 6,199 & 1.32 & 49,554 & 39,819 & .80 & $1,065,790$ & $1,399,593$ & 1.31 \\
\hline 1996 & 331,052 & 489,911 & 1.48 & 20,459 & 16,720 & .82 & 4,855 & 4,083 & .84 & 36,924 & 30,617 & .83 & $1,022,148$ & $1,239,878$ & 1.21 \\
\hline 1997 & 381,109 & 506,695 & 1.33 & 19,094 & 15,927 & .83 & 6,724 & 7,227 & 1.07 & 38,921 & 29,771 & .76 & $1,006,112$ & $1,089,424$ & 1.08 \\
\hline 1998 & 410,369 & 602,187 & 1.47 & 9,130 & 6,924 & .76 & 12,184 & 15,819 & 1.30 & 33,583 & 25,253 & .75 & 981,576 & $1,056,562$ & 1.08 \\
\hline 1999 & 422,801 & 565,797 & 1.34 & 11,114 & 8,320 & .75 & 21,090 & 21,963 & 1.04 & 37,965 & 23,033 & .61 & 971,711 & 955,240 & .98 \\
\hline 2000 & 454,130 & 720,060 & 1.59 & 9,357 & 8,154 & .87 & 25,365 & 36,181 & 1.43 & 37,045 & 28,325 & .76 & 936,611 & $1,101,647$ & 1.18 \\
\hline 2001 & 407,355 & 584,300 & 1.43 & 11,656 & 9,978 & .86 & 30,733 & 43,979 & 1.4 & 53,006 & 36,850 & .70 & 905,072 & 973,783 & 1.08 \\
\hline 2002 & 382,199 & 673,482 & 1.76 & 18,561 & 18,252 & .98 & 26,349 & 42,690 & 1.62 & 40,498 & 33,603 & .83 & 968,128 & $1,290,592$ & 1.33 \\
\hline 2003 & 331,063 & 503,489 & 1.52 & 28,148 & 25,065 & .89 & 28,668 & 38,911 & 1.36 & 49,694 & 36,793 & .74 & 920,496 & $1,065,806$ & 1.16 \\
\hline Average & 333,841 & 511,129 & 1.55 & 21,988 & 21,835 & .92 & 14,948 & 19,808 & 1.25 & 51,352 & 43,742 & .82 & $1,017,255$ & $1,257,382$ & 1.23 \\
\hline Mininum & 186,557 & 336,529 & 1.33 & 9,130 & 6,924 & .75 & 4,709 & 4,083 & .84 & 33,583 & 23,033 & .61 & 905,072 & 955,240 & .98 \\
\hline Maximum & 454,130 & 720,060 & 1.91 & 49,141 & 72,187 & 1.47 & 30,733 & 43,979 & 1.62 & 82,560 & 90,641 & 1.10 & $1,153,416$ & $1,899,155$ & 1.65 \\
\hline Median & 331,063 & 503,489 & 1.52 & 20,033 & 18,252 & .87 & 10,676 & 14,308 & 1.32 & 49,554 & 36,793 & .80 & $1,006,112$ & $1,239,878$ & 1.18 \\
\hline Slope & 21,980 & 28,408 & -.0133 & $-1,813$ & $-1,940$ & -.0108 & 2,011 & 3,213 & .02 & $-2,940$ & $-3,983$ & -.0239 & $-21,939$ & $-53,651$ & .026 \\
\hline p-value & .0018 & .0012 & .300 & .033 & .033 & .392 & .017 & .024 & .179 & .059 & .017 & .006 & 0 & .006 & .065 \\
\hline
\end{tabular}


Table 9. Trends in precipitation and irrigation by system type, crop type, and prorated crop type for Groundwater Management District 3, southwest Kansas, 1991-2003.-Continued

Data from Kansas Water Office and Kansas Department of Agriculture (1993-2005). acre-ft, acre-feet; acre-ft/acre, acre-foot per acre. Blue shading indicates trend is significant at 95-percent level $(\mathrm{p}<0.05)$. Green shading indicates trend is significant at 90-percent level $(\mathrm{p}<0.10)$. NA, data not available]

\begin{tabular}{|c|c|c|c|c|c|c|}
\hline \multirow[b]{2}{*}{ Year } & \multicolumn{6}{|c|}{ Total acres irrigated } \\
\hline & $\begin{array}{l}\text { Prorated } \\
\text { alfalfa }\end{array}$ & $\begin{array}{l}\text { Prorated } \\
\text { corn }\end{array}$ & $\begin{array}{l}\text { Prorated } \\
\text { grain } \\
\text { sorghum }\end{array}$ & $\begin{array}{l}\text { Prorated } \\
\text { soybeans }\end{array}$ & $\begin{array}{c}\text { Prorated } \\
\text { wheat }\end{array}$ & $\begin{array}{c}\text { Other } \\
\text { prorated, } \\
\text { unspecified }\end{array}$ \\
\hline 1991 & 112,077 & 618,784 & 295,696 & 9,440 & 514,787 & $\overline{42,407}$ \\
\hline 1992 & 143,999 & 580,205 & 217,329 & 64,803 & 507,719 & 59,639 \\
\hline 1993 & 144,543 & 661,461 & 147,899 & 49,100 & 508,370 & 36,812 \\
\hline 1994 & 149,518 & 683,368 & 135,429 & 44,789 & 500,057 & 42,404 \\
\hline 1995 & 158,070 & 680,288 & 123,904 & 38,817 & 479,276 & 52,405 \\
\hline 1996 & 155,038 & 719,069 & 137,261 & 28,719 & 438,073 & 45,702 \\
\hline 1997 & 173,314 & 775,920 & 111,611 & 30,728 & 443,212 & 40,549 \\
\hline 1998 & 188,807 & 788,317 & 84,059 & 47,241 & 409,832 & 65,148 \\
\hline 1999 & 179,036 & 791,832 & 83,715 & 73,544 & 394,238 & 72,500 \\
\hline 2000 & 166,767 & 812,764 & 72,984 & 84,988 & 377,381 & 69,658 \\
\hline 2001 & 186,846 & 728,078 & 84,338 & 98,678 & 368,669 & 85,828 \\
\hline 2002 & 211,179 & 708,406 & 116,404 & 78,134 & 377,889 & 102,964 \\
\hline 2003 & 210,287 & 622,657 & 141,593 & 73,613 & 365,540 & 100,909 \\
\hline Average & 167,652 & 705,473 & 134,786 & 55,584 & 437,311 & 62,840 \\
\hline Mininum & 112,077 & 580,205 & 72,984 & 9,440 & 365,540 & 36,812 \\
\hline Maximum & 211,179 & 812,764 & 295,696 & 98,678 & 514,787 & 102,964 \\
\hline Median & 166,767 & 708,406 & 123,904 & 49,100 & 438,073 & 59,639 \\
\hline Slope & 6,646 & 12,608 & $-10,375$ & 4,738 & $-14,611$ & 5,629 \\
\hline p-value & 0 & .044 & .0173 & .0327 & 0 & .003 \\
\hline
\end{tabular}


Table 10. Trends in precipitation and irrigation by system type, crop type, and prorated crop type for Groundwater Management District 4, northwest Kansas, $1991-2003$.

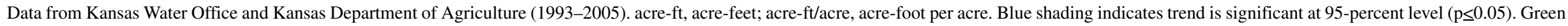
shading indicates trend is significant at 90 -percent level $(\mathrm{p} \leq 0.10)$. NA, data not available]

\begin{tabular}{|c|c|c|c|c|c|c|c|c|c|c|c|c|c|c|c|c|}
\hline \multirow[b]{2}{*}{ Year } & \multirow{2}{*}{$\begin{array}{l}\text { Meteor- } \\
\text { ological } \\
\text { region } 1 \\
\text { precipi- } \\
\text { tation } \\
\text { (inches) }\end{array}$} & \multirow[b]{2}{*}{$\begin{array}{c}\text { Total } \\
\text { acres } \\
\text { irrigated }\end{array}$} & \multirow[b]{2}{*}{$\begin{array}{c}\text { Total } \\
\text { irrigation } \\
\text { water use } \\
\text { (acre-ft) }\end{array}$} & \multirow[b]{2}{*}{$\begin{array}{c}\text { Average } \\
\text { rate of } \\
\text { irrigation } \\
\text { (acre- } \\
\text { ft/yr) }\end{array}$} & \multicolumn{3}{|c|}{ Flood irrigation } & \multicolumn{3}{|c|}{ Center pivot irrigation } & \multicolumn{3}{|c|}{$\begin{array}{l}\text { Center pivot irrigation with drop } \\
\text { nozzle }\end{array}$} & \multicolumn{3}{|c|}{ Alfalfa } \\
\hline & & & & & $\begin{array}{l}\text { Total acres } \\
\text { irrigated }\end{array}$ & $\begin{array}{c}\text { Water } \\
\text { used } \\
\text { (acre-ft) }\end{array}$ & $\begin{array}{c}\text { Rate of } \\
\text { irriga- } \\
\text { tion } \\
\text { (acre- } \\
\text { ft/acre) }\end{array}$ & $\begin{array}{c}\text { Total } \\
\text { acres } \\
\text { irrigated }\end{array}$ & $\begin{array}{c}\text { Water } \\
\text { used } \\
\text { (acre-ft) }\end{array}$ & $\begin{array}{c}\text { Rate of } \\
\text { irriga- } \\
\text { tion } \\
\text { (acre- } \\
\text { ft/acre) }\end{array}$ & $\begin{array}{c}\text { Total } \\
\text { acres } \\
\text { irrigated }\end{array}$ & $\begin{array}{c}\text { Water } \\
\text { used } \\
\text { (acre-ft) }\end{array}$ & $\begin{array}{c}\text { Rate of } \\
\text { irriga- } \\
\text { tion } \\
\text { (acre- } \\
\text { ft/acre) }\end{array}$ & $\begin{array}{c}\text { Total } \\
\text { acres } \\
\text { irri- } \\
\text { gated }\end{array}$ & $\begin{array}{l}\text { Irrigation } \\
\text { water } \\
\text { used } \\
\text { (acre-ft) }\end{array}$ & $\begin{array}{c}\text { Rate of } \\
\text { irri- } \\
\text { gation } \\
\text { (acre- } \\
\text { ft/acre) }\end{array}$ \\
\hline 1991 & 22.67 & 360,109 & 478,854 & 1.33 & 111,066 & 173,659 & 1.56 & 218,417 & 263,077 & 1.20 & NA & NA & NA & 6,383 & 9,317 & 1.46 \\
\hline 1992 & 25.39 & 340,522 & 268,217 & .79 & 83,917 & 82,776 & .99 & 186,421 & 133,135 & .71 & 43,379 & 31,187 & .72 & 5,237 & 4,765 & .91 \\
\hline 1993 & 30.09 & 343,660 & 261,648 & .76 & 77,960 & 79,034 & 1.01 & 180,509 & 127,103 & .70 & 60,882 & 38,516 & .63 & 6,289 & 4,808 & .76 \\
\hline 1994 & 20.70 & 367,070 & 396,603 & 1.08 & 74,031 & 97,211 & 1.31 & 184,188 & 191,807 & 1.04 & 73,774 & 73,634 & 1.00 & 5,676 & 5,800 & 1.02 \\
\hline 1995 & 22.90 & 355,259 & 361,778 & 1.02 & 60,157 & 73,850 & 1.23 & 195,033 & 193,008 & .99 & 74,158 & 69,698 & .94 & 7,402 & 7,129 & .96 \\
\hline 1996 & 25.85 & 370,641 & 364,688 & .98 & 52,973 &, 599 & 1.18 & 199,944 & 192 & .96 & 85,604 & 78,055 & .91 & 7,200 & 6,499 & .90 \\
\hline 1997 & 19.98 & 381,935 & 413,066 & 1.08 & 44,908 & 54,950 & 1.22 & 136,971 & 142,795 & 1.04 & 170,738 & 184,679 & 1.08 & 8,309 & 9,095 & 1.09 \\
\hline 1998 & 20.87 & 380,712 & 376,852 & .99 & 32,801 & 36,220 & 1.10 & 104,022 & 101,738 & .98 & 212,541 & 209,301 & .98 & 9,299 & 10,419 & 1.12 \\
\hline 1999 & 21.73 & 378,827 & 331,370 & .87 & 26,988 & 28,555 & 1.06 & 95,372 & 80,905 & .85 & 219,918 & 189,915 & .86 & 9,552 & 8,836 & .93 \\
\hline 2000 & 17.38 & 374,945 & 472,688 & 1.26 & 20,792 & 30,969 & 1.49 & 70,750 & 86,909 & 1.23 & 250,894 & 316,092 & 1.26 & 7,897 & 9,928 & 1.26 \\
\hline 2001 & 20.48 & 367,627 & 402,236 & 1.09 & 16,830 & 20,649 & 1.23 & 57,955 & 64,016 & 1.10 & 260,665 & 281,731 & 1.08 & 8,702 & 11,094 & 1.27 \\
\hline 2002 & 11.46 & 374,371 & 500,716 & 1.34 & 14,672 & 22,270 & 1.52 & 50,587 & 66,332 & 1.31 & 275,019 & 368,947 & 1.34 & 8,801 & 13,601 & 1.55 \\
\hline 2003 & 16.06 & 375,994 & 460,830 & 1.23 & 11,610 & 16,227 & 1.40 & 49,425 & 57,226 & 1.16 & 283,226 & 349,235 & 1.23 & 10,240 & 13,600 & 1.33 \\
\hline Average & 21.20 & 367,052 & 391,504 & 1.06 & 48,362 & 59,921 & 1.25 & 133,046 & 130,831 & 1.02 & 167,567 & 182,583 & 1.00 & 7,768 & 8,838 & 1.12 \\
\hline Mininum & 11.46 & 340,522 & 261,648 & .76 & 11,610 & 16,227 & .99 & 49,425 & 57,226 & .70 & 43,379 & 31,187 & .63 & 5,237 & 4,765 & .76 \\
\hline Maximum & 30.09 & 381,935 & 500,716 & 1.34 & 111,066 & 173,659 & 1.56 & 218,417 & 263,077 & 1.31 & 283,226 & 368,947 & 1.34 & 10,240 & 13,601 & 1.55 \\
\hline Median & 20.87 & 370,641 & 396,603 & 1.08 & 44,908 & 54,950 & 1.23 & 136,971 & 127,103 & 1.04 & 191,640 & 187,297 & .99 & 7,897 & 9,095 & 1.09 \\
\hline Slope & -.84 & 2,330 & 12,531 & .03 & $-7,837$ & $-7,752$ & .0191 & $-15,115$ & $-14,607$ & .0282 & 24,366 & 33,764 & .05 & 355 & 782 & .04 \\
\hline $\mathrm{p}$-value & .009 & .044 & .100 & .321 & 0 & 0 & .328 & 0 & .001 & .346 & 0 & 0 & .013 & .001 & .001 & .033 \\
\hline
\end{tabular}


Table 10. Trends in precipitation and irrigation by system type, crop type, and prorated crop type for Groundwater Management District 4, northwest Kansas, 19912003.-Continued

Data from Kansas Water Office and Kansas Department of Agriculture (1993-2005). acre-ft, acre-feet; acre-ft/acre, acre-foot per acre. Blue shading indicates trend is significant at 95-percent level ( $\mathrm{p} \leq 0.05$ ). Green shading indicates trend is significant at 90 -percent level $(\mathrm{p} \leq 0.10)$. NA, data not available

\begin{tabular}{|c|c|c|c|c|c|c|c|c|c|c|c|c|c|c|c|}
\hline \multirow[b]{2}{*}{ Year } & \multicolumn{3}{|c|}{ Corn } & \multicolumn{3}{|c|}{ Grain sorghum } & \multicolumn{3}{|c|}{ Soybeans } & \multicolumn{3}{|c|}{ Wheat } & \multicolumn{3}{|c|}{ Other/multiple use } \\
\hline & $\begin{array}{c}\text { Total } \\
\text { acres } \\
\text { irrigated }\end{array}$ & $\begin{array}{l}\text { Irrigation } \\
\text { water } \\
\text { used } \\
\text { (acre-ft) }\end{array}$ & $\begin{array}{c}\text { Rate of } \\
\text { irriga- } \\
\text { tion } \\
\text { (acre- } \\
\text { ft/acre) }\end{array}$ & $\begin{array}{c}\text { Total } \\
\text { acres } \\
\text { irrigated }\end{array}$ & $\begin{array}{l}\text { Irrigation } \\
\text { water } \\
\text { used } \\
\text { (acre-ft) }\end{array}$ & $\begin{array}{l}\text { Rate of } \\
\text { irrigation } \\
\text { (acre- } \\
\mathrm{ft} / \text { acre) }\end{array}$ & $\begin{array}{c}\text { Total } \\
\text { acres } \\
\text { irrigated }\end{array}$ & $\begin{array}{l}\text { Irrigation } \\
\text { water } \\
\text { used } \\
\text { (acre-ft) }\end{array}$ & $\begin{array}{l}\text { Rate of } \\
\text { irrigation } \\
\text { (acre- } \\
\text { ft/acre) }\end{array}$ & $\begin{array}{c}\text { Total } \\
\text { acres } \\
\text { irrigated }\end{array}$ & $\begin{array}{l}\text { Irrigation } \\
\text { water } \\
\text { used } \\
\text { (acre-ft) }\end{array}$ & $\begin{array}{l}\text { Rate of } \\
\text { irrigation } \\
\text { (acre- } \\
\mathrm{ft} / \text { acre) }\end{array}$ & $\begin{array}{c}\text { Total } \\
\text { acres } \\
\text { irrigated }\end{array}$ & $\begin{array}{l}\text { Irrigation } \\
\text { waterused } \\
\text { (acre-ft) }\end{array}$ & $\begin{array}{c}\text { Rate of } \\
\text { irriga- } \\
\text { tion } \\
\text { (acre- } \\
\text { ft/acre) }\end{array}$ \\
\hline 1991 & 140,359 & 212,020 & 1.51 & 23,474 & 28,699 & 1.22 & 5,604 & 6,630 & 1.18 & 15,816 & 11,209 & 0.71 & 168,473 & 210,980 & 1.25 \\
\hline 1992 & 139,908 & 119,470 & .85 & 28,445 & 20,129 & .71 & 6,996 & 4,705 & .67 & 15,184 & 8,169 & .54 & 144,752 & 110,979 & .77 \\
\hline 1993 & 164,651 & 139,859 & .85 & 6,759 & 3,405 & .50 & 4,489 & 2,536 & .57 & 16,204 & 7,811 & .48 & 145,268 & 103,228 & .71 \\
\hline 1994 & 177,265 & 218,340 & 1.23 & 7,125 & 5,941 & .83 & 3,929 & 3,709 & .94 & 11,625 & 6,803 & .59 & 161,450 & 156,009 & .97 \\
\hline 1995 & 158,766 & 195,690 & 1.23 & 8,364 & 7,067 & .84 & 3,576 & 3,495 & .98 & 17,480 & 8,001 & .46 & 159,671 & 140,396 & .88 \\
\hline 1996 & 204,159 & 224,076 & 1.10 & 6,333 & 4,133 & .65 & 2,504 & 2,264 & .90 & 13,580 & 7,217 & .53 & 136,865 & 120,498 & .88 \\
\hline 1997 & 222,164 & 265,040 & 1.19 & 5,745 & 4,360 & .76 & 6,348 & 6,523 & 1.03 & 13,982 & 8,530 & .61 & 125,387 & 119,518 & .95 \\
\hline 1998 & 219,731 & 240,991 & 1.10 & 4,483 & 2,998 & .67 & 10,096 & 8,950 & .89 & 9,518 & 5,203 & .55 & 127,585 & 108,291 & .85 \\
\hline 1999 & 215,832 & 209,552 & .97 & 3,479 & 2,196 & .63 & 12,014 & 9,166 & .76 & 11,810 & 6,000 & .51 & 126,140 & 95,620 & .76 \\
\hline 2000 & 215,444 & 298,766 & 1.39 & 2,526 & 2,126 & .84 & 15,063 & 18,755 & 1.25 & 9,165 & 5,391 & .59 & 124,850 & 137,721 & 1.10 \\
\hline 2001 & 185,803 & 233,083 & 1.25 & 6,159 & 5,871 & .95 & 19,852 & 21,553 & 1.09 & 12,662 & 6,726 & .53 & 134,449 & 123,908 & .92 \\
\hline 2002 & 178,199 & 262,899 & 1.48 & 4,393 & 4,553 & 1.04 & 18,856 & 26,376 & 1.40 & 8,869 & 6,056 & .68 & 155,253 & 187,230 & 1.21 \\
\hline 2003 & 136,095 & 198,494 & 1.46 & 8,313 & 8,448 & 1.02 & 15,726 & 19,700 & 1.25 & 21,318 & 13,773 & .65 & 184,302 & 206,816 & 1.12 \\
\hline Average & 181,414 & 216,791 & 1.20 & 8,892 & 7,687 & .82 & 9,619 & 10,336 & .99 & 13,632 & 7,761 & .57 & 145,727 & 140,092 & .95 \\
\hline Mininum & 136,095 & 119,470 & .85 & 2,526 & 2,126 & .50 & 2,504 & 2,264 & .57 & 8,869 & 5,203 & .46 & 124,850 & 95,620 & .71 \\
\hline Maximum & 222,164 & 298,766 & 1.51 & 28,445 & 28,699 & 1.22 & 19,852 & 26,376 & 1.40 & 21,318 & 13,773 & .71 & 184,302 & 210,980 & 1.25 \\
\hline Median & 178,199 & 218,340 & 1.23 & 6,333 & 4,553 & .83 & 6,996 & 6,630 & .98 & 13,580 & 7,217 & .55 & 144,752 & 123,908 & .92 \\
\hline Slope & 3,634 & 6,351 & .0306 & -694 & -547 & .022 & 1,389 & 1,736 & .039 & -426 & -211 & .0067 & $-1,359$ & 1,267 & .022 \\
\hline p-value & .4277 & .0768 & .177 & .017 & .200 & .271 & .012 & .006 & .067 & .161 & .200 & .501 & .360 & .760 & .32 \\
\hline
\end{tabular}


Table 10. Trends in precipitation and irrigation by system type, crop type, and

prorated crop type for Groundwater Management District 4, northwest Kansas, 1991-2003.-Continued

Data from Kansas Water Office and Kansas Department of Agriculture (1993-2005). acre-ft, acre-feet; acre-ft/acre, acre-foot per acre. Blue shading indicates trend is significant at 95-percent level $(\mathrm{p} \leq 0.05)$. Green shading indicates trend is significant at 90 -percent level $(\mathrm{p} \leq 0.10)$. NA, data not available]

\begin{tabular}{|c|c|c|c|c|c|c|}
\hline \multirow[b]{2}{*}{ Year } & \multicolumn{6}{|c|}{ Total acres irrigated } \\
\hline & $\begin{array}{l}\text { Prorated } \\
\text { alfalfa }\end{array}$ & $\begin{array}{l}\text { Prorated } \\
\text { corn }\end{array}$ & $\begin{array}{c}\text { Prorated } \\
\text { grain } \\
\text { sorghum }\end{array}$ & $\begin{array}{l}\text { Prorated } \\
\text { soybeans }\end{array}$ & $\begin{array}{c}\text { Prorated } \\
\text { wheat }\end{array}$ & $\begin{array}{c}\text { Other } \\
\text { prorated, } \\
\text { unspecified }\end{array}$ \\
\hline 1991 & 6,383 & 140,359 & 23,474 & 5,604 & 15,816 & 12,615 \\
\hline 1992 & 9,789 & 190,406 & 40,418 & 18,279 & 47,507 & 34,124 \\
\hline 1993 & 10,619 & 216,276 & 12,412 & 15,016 & 49,043 & 40,293 \\
\hline 1994 & 10,680 & 233,777 & 13,749 & 12,615 & 45,981 & 50,268 \\
\hline 1995 & 11,843 & 209,783 & 16,093 & 11,148 & 48,370 & 58,022 \\
\hline 1996 & 13,805 & 255,858 & 15,452 & 8,851 & 45,665 & 31,009 \\
\hline 1997 & 13,904 & 268,921 & 11,708 & 15,719 & 44,398 & 27,285 \\
\hline 1998 & 16,467 & 266,057 & 9,282 & 24,606 & 37,293 & 27,007 \\
\hline 1999 & 16,161 & 257,917 & 6,921 & 25,968 & 35,943 & 35,917 \\
\hline 2000 & 13,889 & 260,474 & 4,729 & 33,642 & 34,192 & 28,020 \\
\hline 2001 & 14,260 & 230,022 & 9,784 & 37,926 & 40,338 & 35,298 \\
\hline 2002 & 15,838 & 234,348 & 10,409 & 47,459 & 42,142 & 24,175 \\
\hline 2003 & 17,613 & 193,409 & 18,107 & 43,128 & 67,008 & 36,729 \\
\hline Average & 13,173 & 227,508 & 14,811 & 23,074 & 42,592 & 33,905 \\
\hline Mininum & 6,383 & 140,359 & 4,729 & 5,604 & 15,816 & 12,615 \\
\hline Maximum & 17,613 & 268,921 & 40,418 & 47,459 & 67,008 & 58,022 \\
\hline Median & 13,889 & 233,777 & 12,412 & 18,279 & 44,398 & 34,124 \\
\hline Slope & 777 & 4,397 & $-1,107$ & 3,197 & -532 & -332 \\
\hline p-value & 0 & .200 & .0585 & .0012 & .502 & .855 \\
\hline
\end{tabular}


Table 11. Trends in precipitation and irrigation by system type, crop type, and prorated crop type for Groundwater Management District 5, south-central Kansas, $1991-2003$.

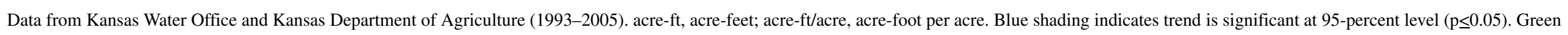
shading indicates trend is significant at 90 -percent level $(\mathrm{p} \leq 0.10)$. NA, data not available]

\begin{tabular}{|c|c|c|c|c|c|c|c|c|c|c|c|c|c|c|c|c|}
\hline \multirow[b]{2}{*}{ Year } & \multirow{2}{*}{$\begin{array}{l}\text { Meteor- } \\
\text { ological } \\
\text { region } 8 \\
\text { precipi- } \\
\text { tation } \\
\text { (inches) }\end{array}$} & \multirow[b]{2}{*}{$\begin{array}{c}\text { Total } \\
\text { acres } \\
\text { irrigated }\end{array}$} & \multirow[b]{2}{*}{$\begin{array}{c}\text { Total } \\
\text { irrigation } \\
\text { water use } \\
\text { (acre-ft) }\end{array}$} & \multirow[b]{2}{*}{ Average } & \multicolumn{3}{|c|}{ Flood irrigation } & \multicolumn{3}{|c|}{ Center pivot irrigation } & \multicolumn{3}{|c|}{$\begin{array}{l}\text { Center pivot irrigation with drop } \\
\text { nozzle }\end{array}$} & \multicolumn{3}{|c|}{ Alfalfa } \\
\hline & & & & & $\begin{array}{c}\text { Total } \\
\text { acres } \\
\text { irrigated }\end{array}$ & $\begin{array}{c}\text { Water } \\
\text { used } \\
\text { (acre-ft) }\end{array}$ & $\begin{array}{c}\text { Rate of } \\
\text { irriga- } \\
\text { tion } \\
\text { (acre- } \\
\mathrm{ft} / \text { acre) }\end{array}$ & $\begin{array}{c}\text { Total } \\
\text { acres } \\
\text { irrigated }\end{array}$ & $\begin{array}{l}\text { Water used } \\
\text { (acre-ft) }\end{array}$ & $\begin{array}{c}\text { Rate of } \\
\text { irriga- } \\
\text { tion } \\
\text { (acre- } \\
\mathrm{ft} / \text { acre) }\end{array}$ & $\begin{array}{c}\text { Total } \\
\text { acres } \\
\text { irrigated }\end{array}$ & $\begin{array}{c}\text { Water } \\
\text { used } \\
\text { (acre-ft) }\end{array}$ & $\begin{array}{l}\text { Rate of } \\
\text { irriga- } \\
\text { tion } \\
\text { (acre- } \\
\text { ft/acre) }\end{array}$ & $\begin{array}{c}\text { Total } \\
\text { acres irri- } \\
\text { gated }\end{array}$ & $\begin{array}{l}\text { Irrigation } \\
\text { waterused } \\
\text { (acre-ft) }\end{array}$ & $\begin{array}{l}\text { Rate of } \\
\text { irri- } \\
\text { gation } \\
\text { (acre- } \\
\text { ft/acre) }\end{array}$ \\
\hline 1991 & 22.72 & 434,745 & 606,672 & 1.40 & 66,906 & 98,928 & 1.48 & 358,046 & 494,995 & 1.38 & NA & NA & NA & 47,721 & 68,609 & 1.44 \\
\hline 1992 & 31.96 & 418,749 & 330,406 & .79 & 53,548 & 34,700 & .65 & 342,227 & 278,906 & .81 & 15,919 & 11,997 & .75 & 42,745 & 37,283 & .87 \\
\hline 1993 & 34.08 & 410,330 & 308,474 & .75 & 48,087 & 27,207 & .57 & 346,260 & 268,263 & .77 & 8,495 & 8,095 & .95 & 40,900 & 28,089 & .69 \\
\hline 1994 & 22.06 & 428,022 & 565,785 & 1.32 & 56,293 & 73,273 & 1.30 & 352,781 & 469,318 & 1.33 & 9,305 & 12,269 & 1.32 & 41,386 & 56,319 & 1.36 \\
\hline 1995 & 31.66 & 426,810 & 453,515 & 1.06 & 57,069 & 56,766 & .99 & 348,025 & 375,065 & 1.08 & 14,367 & 14,473 & 1.01 & 40,244 & 37,877 & .94 \\
\hline 1996 & 32.01 & 431,379 & 371,749 & .86 & 49,876 & 41,465 & .83 & 355,894 & 309,488 & .87 & 18,249 & 15,647 & .86 & 37,692 & 29,566 & .78 \\
\hline 1997 & 34.39 & 439,038 & 341,855 & .78 & 46,642 & 34,089 & .73 & 269,480 & 211,070 & .78 & 115,490 & 91,938 & .80 & 37,009 & 27,488 & .74 \\
\hline 1998 & 30.14 & 448,803 & 511,214 & 1.14 & 43,939 & 41,606 & .95 & 219,963 & 254,895 & 1.16 & 167,639 & 194,692 & 1.16 & 36,064 & 41,255 & 1.14 \\
\hline 1999 & 32.17 & 457,878 & 463,473 & 1.01 & 42,592 & 35,707 & .84 & 209,721 & 217,124 & 1.04 & 189,085 & 195,702 & 1.03 & 36,481 & 32,904 & .90 \\
\hline 2000 & 32.76 & 458,336 & 528,801 & 1.15 & 38,213 & 41,422 & 1.08 & 174,385 & 204,296 & 1.17 & 229,741 & 265,459 & 1.16 & 30,025 & 34,944 & 1.16 \\
\hline 2001 & 24.87 & 462,003 & 567,745 & 1.23 & 39,014 & 41,734 & 1.07 & 143,220 & 176,108 & 1.23 & 258,986 & 325,155 & 1.26 & 32,947 & 39,317 & 1.19 \\
\hline 2002 & 29.41 & 464,945 & 571,254 & 1.23 & 35,692 & 39,650 & 1.11 & 118,264 & 145,967 & 1.23 & 290,459 & 360,588 & 1.24 & 42,363 & 54,769 & 1.29 \\
\hline 2003 & 28.41 & 456,932 & 575,807 & 1.26 & 30,817 & 39,546 & 1.28 & 99,141 & 126,458 & 1.28 & 307,325 & 385,951 & 1.26 & 44,433 & 50,458 & 1.14 \\
\hline Average & 29.74 & 441,382 & 476,673 & 1.08 & 46,822 & 46,623 & .99 & 256,724 & 271,689 & 1.09 & 135,422 & 156,831 & 1.07 & 39,232 & 41,452 & 1.05 \\
\hline Mininum & 22.06 & 410,330 & 308,474 & .75 & 30,817 & 27,207 & .57 & 99,141 & 126,458 & .77 & 8,495 & 8,095 & .75 & 30,025 & 27,488 & .69 \\
\hline Maximum & 34.39 & 464,945 & 606,672 & 1.40 & 66,906 & 98,928 & 1.48 & 358,046 & 494,995 & 1.38 & 307,325 & 385,951 & 1.32 & 47,721 & 68,609 & 1.44 \\
\hline Median & 31.66 & 439,038 & 511,214 & 1.14 & 46,642 & 41,422 & .99 & 269,480 & 254,895 & 1.16 & 141,565 & 143,315 & 1.10 & 40,244 & 37,877 & 1.14 \\
\hline Slope & -.14 & 4,617 & 16,244 & .028 & $-2,630$ & -357 & .04 & $-25,018$ & $-26,280$ & .025 & 31,401 & 39,898 & .03 & -970 & 366 & .025 \\
\hline $\mathrm{p}$-value & .951 & .001 & .059 & .222 & 0 & .502 & .246 & 0 & 0 & .222 & 0 & 0 & .033 & .059 & .077 & .463 \\
\hline
\end{tabular}


Table 11. Trends in precipitation and irrigation by system type, crop type, and prorated crop type for Groundwater Management District 5, south-central Kansas, 19912003.-Continued

Data from Kansas Water Office and Kansas Department of Agriculture (1993-2005). acre-ft, acre-feet; acre-ft/acre, acre-foot per acre. Blue shading indicates trend is significant at 95-percent level ( $\mathrm{p} \leq 0.05$ ). Green shading indicates trend is significant at 90 -percent level $(\mathrm{p} \leq 0.10)$. NA, data not available]

\begin{tabular}{|c|c|c|c|c|c|c|c|c|c|c|c|c|c|c|c|}
\hline \multirow[b]{2}{*}{ Year } & \multicolumn{3}{|c|}{ Corn } & \multicolumn{3}{|c|}{ Grain sorghum } & \multicolumn{3}{|c|}{ Soybeans } & \multicolumn{3}{|c|}{ Wheat } & \multicolumn{3}{|c|}{ Other/multiple use } \\
\hline & $\begin{array}{c}\text { Total } \\
\text { acres } \\
\text { irrigated }\end{array}$ & $\begin{array}{l}\text { Irrigation } \\
\text { water } \\
\text { used } \\
\text { (acre-ft) }\end{array}$ & $\begin{array}{c}\text { Rate of } \\
\text { irriga- } \\
\text { tion } \\
\text { (acre- } \\
\text { ft/acre) }\end{array}$ & $\begin{array}{c}\text { Total } \\
\text { acres } \\
\text { irrigated }\end{array}$ & $\begin{array}{l}\text { Irrigation } \\
\text { water } \\
\text { used } \\
\text { (acre-ft) }\end{array}$ & $\begin{array}{c}\text { Rate of } \\
\text { irriga- } \\
\text { tion } \\
\text { (acre- } \\
\mathrm{ft} / \mathrm{acre} \text { ) }\end{array}$ & $\begin{array}{c}\text { Total } \\
\text { acres } \\
\text { irrigated }\end{array}$ & $\begin{array}{l}\text { Irrigation } \\
\text { water } \\
\text { used } \\
\text { (acre-ft) }\end{array}$ & $\begin{array}{l}\text { Rate of } \\
\text { irriga- } \\
\text { tion } \\
\text { (acre- } \\
\text { ft/acre) }\end{array}$ & $\begin{array}{c}\text { Total acres } \\
\text { irrigated }\end{array}$ & $\begin{array}{l}\text { Irrigation } \\
\text { water } \\
\text { used } \\
\text { (acre-ft) }\end{array}$ & $\begin{array}{l}\text { Rate of } \\
\text { irriga- } \\
\text { tion } \\
\text { (acre- } \\
\text { ft/acre) }\end{array}$ & $\begin{array}{c}\text { Total acres } \\
\text { irrigated }\end{array}$ & $\begin{array}{l}\text { Irrigation } \\
\text { water } \\
\text { used } \\
\text { (acre-ft) }\end{array}$ & $\begin{array}{l}\text { Rate of } \\
\text { irriga- } \\
\text { tion } \\
\text { (acre- } \\
\text { ft/acre) }\end{array}$ \\
\hline 1991 & 158,182 & 232,206 & 1.47 & 18,691 & 21,402 & 1.15 & 29,079 & 40,321 & 1.39 & 15,114 & 10,958 & 0.73 & 165,958 & 233,176 & 1.41 \\
\hline 1992 & 180,103 & 152,136 & .84 & 18,262 & 9,483 & .52 & 21,307 & 16,609 & .78 & 14,586 & 7,734 & .53 & 141,746 & 107,162 & .76 \\
\hline 1993 & 184,608 & 158,642 & .86 & 12,528 & 5,227 & .42 & 23,824 & 18,912 & .79 & 11,746 & 3,497 & .30 & 136,724 & 94,107 & .69 \\
\hline 1994 & 189,124 & 264,327 & 1.40 & 11,889 & 11,158 & .94 & 29,119 & 38,466 & 1.32 & 7,308 & 5,613 & .77 & 149,196 & 189,901 & 1.27 \\
\hline 1995 & 185,095 & 221,508 & 1.20 & 14,059 & 10,447 & .74 & 28,128 & 31,444 & 1.12 & 10,071 & 4,496 & .45 & 149,213 & 147,743 & .99 \\
\hline 1996 & 210,359 & 200,650 & .95 & 14,229 & 8,771 & .62 & 31,627 & 25,044 & .79 & 5,985 & 2,522 & .42 & 131,487 & 105,197 & .80 \\
\hline 1997 & 200,050 & 170,569 & .85 & 10,588 & 6,042 & .57 & 54,749 & 42,507 & .78 & 6,481 & 2,322 & .36 & 130,161 & 92,926 & .71 \\
\hline 1998 & 203,016 & 245,091 & 1.21 & 7,602 & 5,766 & .76 & 66,447 & 78,408 & 1.18 & 5,238 & 1,969 & .38 & 130,436 & 138,726 & 1.06 \\
\hline 1999 & 197,704 & 213,070 & 1.08 & 6,840 & 5,358 & .78 & 72,459 & 80,734 & 1.11 & 9,279 & 4,386 & .47 & 135,115 & 127,021 & .94 \\
\hline 2000 & 207,135 & 243,038 & 1.17 & 6,149 & 5,302 & .86 & 73,072 & 91,160 & 1.25 & 7,934 & 4,503 & .57 & 134,021 & 149,854 & 1.12 \\
\hline 2001 & 201,073 & 259,856 & 1.29 & 7,112 & 6,394 & .90 & 67,948 & 90,203 & 1.33 & 9,696 & 6,164 & .64 & 143,227 & 165,810 & 1.16 \\
\hline 2002 & 201,842 & 265,484 & 1.32 & 8,081 & 7,921 & .98 & 67,327 & 86,427 & 1.28 & 9,024 & 5,536 & .61 & 136,308 & 151,117 & 1.11 \\
\hline 2003 & 192,626 & 266,588 & 1.38 & 16,136 & 16,735 & 1.04 & 57,229 & 77,069 & 1.35 & 12,339 & 7,157 & .58 & 134,169 & 157,801 & 1.18 \\
\hline Average & 193,147 & 222,551 & 1.16 & 11,705 & 9,231 & .79 & 47,870 & 55,177 & 1.11 & 9,600 & 5,143 & .52 & 139,828 & 143,119 & 1.02 \\
\hline Mininum & 158,182 & 152,136 & .84 & 6,149 & 5,227 & .42 & 21,307 & 16,609 & .78 & 5,238 & 1,969 & .30 & 130,161 & 92,926 & .69 \\
\hline Maximum & 210,359 & 266,588 & 1.47 & 18,691 & 21,402 & 1.15 & 73,072 & 91,160 & 1.39 & 15,114 & 10,958 & .77 & 165,958 & 233,176 & 1.41 \\
\hline Median & 197,704 & 232,206 & 1.20 & 11,889 & 7,921 & .78 & 54,749 & 42,507 & 1.18 & 9,279 & 4,503 & .53 & 136,308 & 147,743 & 1.06 \\
\hline Slope & 2,256 & 6,448 & .03 & -901 & -342 & .042 & 4,092 & 5,788 & .031 & -289 & -40 & .01 & -981 & 2,924 & .024 \\
\hline $\mathrm{p}$-value & .0239 & .0173 & .256 & .033 & .360 & .410 & .003 & .006 & .159 & .360 & .855 & .583 & .2001 & .502 & .2996 \\
\hline
\end{tabular}


Table 11. Trends in precipitation and irrigation by system type, crop type, and prorated crop type for Groundwater Management District 5, south-central Kansas, 1991-2003.-Continued

Data from Kansas Water Office and Kansas Department of Agriculture (1993-2005). acre-ft, acre-feet; acre-ft/acre, acre-foot per acre. Blue shading indicates trend is significant at 95-percent level $(\mathrm{p} \leq 0.05)$. Green shading indicates trend is significant at 90-percent level $(\mathrm{p} \leq 0.10)$. NA, data not available]

\begin{tabular}{|c|c|c|c|c|c|c|}
\hline \multirow[b]{2}{*}{ Year } & \multicolumn{6}{|c|}{ Total acres irrigated } \\
\hline & $\begin{array}{c}\text { Prorated } \\
\text { alfalfa }\end{array}$ & $\begin{array}{l}\text { Prorated } \\
\text { corn }\end{array}$ & $\begin{array}{l}\text { Prorated } \\
\text { grain } \\
\text { sorghum }\end{array}$ & $\begin{array}{l}\text { Prorated } \\
\text { soybeans }\end{array}$ & $\begin{array}{c}\text { Prorated } \\
\text { wheat }\end{array}$ & $\begin{array}{c}\text { Other } \\
\text { prorated, } \\
\text { unspecified }\end{array}$ \\
\hline 1991 & 47,721 & 158,182 & 18,691 & 29,079 & 15,114 & 7,861 \\
\hline 1992 & 55,764 & 214,451 & 39,003 & 49,966 & 44,489 & 14,457 \\
\hline 1993 & 51,262 & 225,998 & 26,430 & 55,657 & 35,792 & 15,156 \\
\hline 1994 & 54,559 & 234,543 & 30,455 & 62,261 & 32,593 & 13,611 \\
\hline 1995 & 52,364 & 229,328 & 30,637 & 63,554 & 36,299 & 14,629 \\
\hline 1996 & 48,493 & 250,564 & 28,744 & 64,626 & 26,268 & 12,684 \\
\hline 1997 & 48,852 & 237,235 & 23,958 & 89,570 & 26,788 & 12,634 \\
\hline 1998 & 46,658 & 242,618 & 16,866 & 103,000 & 21,146 & 18,515 \\
\hline 1999 & 46,243 & 238,976 & 15,353 & 108,083 & 26,906 & 22,318 \\
\hline 2000 & 39,576 & 247,618 & 13,849 & 109,242 & 27,891 & 20,160 \\
\hline 2001 & 41,862 & 241,776 & 14,994 & 105,126 & 31,520 & 26,725 \\
\hline 2002 & 53,780 & 238,746 & 17,000 & 101,677 & 29,178 & 24,564 \\
\hline 2003 & 55,165 & 223,616 & 27,714 & 86,835 & 37,357 & 26,245 \\
\hline Average & 49,408 & 229,512 & 23,361 & 79,129 & 30,103 & 17,658 \\
\hline Mininum & 39,576 & 158,182 & 13,849 & 29,079 & 15,114 & 7,861 \\
\hline Maximum & 55,764 & 250,564 & 39,003 & 109,242 & 44,489 & 26,725 \\
\hline Median & 48,852 & 237,235 & 23,958 & 86,835 & 29,178 & 15,156 \\
\hline Slope & -676 & 2,118 & $-1,466$ & 6,161 & 184 & 1,367 \\
\hline p-value & .300 & .077 & .0585 & .0012 & .760 & .004 \\
\hline
\end{tabular}


Table 12. Trends in precipitation and irrigation by system type, crop type, and prorated crop type for all Groundwater Management Districts, Kansas, $1991-2003$.

Data from Kansas Water Office and Kansas Department of Agriculture (1993-2005). acre-ft, acre-feet; acre-ft/acre, acre-foot per acre. Blue shading indicates trend is significant at 95-percent level ( $\mathrm{p} \leq 0.05$ ). Green shading indicates trend is significant at 90 -percent level $(\mathrm{p} \leq 0.10)$. NA, data not available]

\begin{tabular}{|c|c|c|c|c|c|c|c|c|c|c|c|c|c|}
\hline \multirow[b]{2}{*}{ Year } & \multirow{2}{*}{$\begin{array}{c}\text { Average } \\
\text { precipi- } \\
\text { tation for } \\
\text { meteoro- } \\
\text { logical } \\
\text { regions } \\
1,4,7 \\
\text { and } 8 \\
\text { (inches) }\end{array}$} & \multirow[b]{2}{*}{$\begin{array}{c}\text { Total } \\
\text { acres } \\
\text { irrigated }\end{array}$} & \multirow[b]{2}{*}{$\begin{array}{c}\text { Total } \\
\text { irrigation } \\
\text { water use } \\
\text { (acre-ft) }\end{array}$} & \multirow[b]{2}{*}{$\begin{array}{l}\text { Average } \\
\text { rate of } \\
\text { irrigation } \\
\text { (acre- } \\
\mathrm{ft} / \text { acre) }\end{array}$} & \multicolumn{3}{|c|}{ Flood irrigation } & \multicolumn{3}{|c|}{ Center pivot irrigation } & \multicolumn{3}{|c|}{ Center pivot irrigation with drop nozzle } \\
\hline & & & & & $\begin{array}{l}\text { Total acres } \\
\text { irrigated }\end{array}$ & $\begin{array}{l}\text { Waterused } \\
\text { (acre-ft) }\end{array}$ & $\begin{array}{c}\text { Rate of } \\
\text { irrigation } \\
\text { (acre- } \\
\text { ft/acre) }\end{array}$ & $\begin{array}{l}\text { Total acres } \\
\text { irrigated }\end{array}$ & $\begin{array}{c}\text { Water used } \\
\text { (acre-ft) }\end{array}$ & $\begin{array}{c}\text { Rate of } \\
\text { irrigation } \\
\text { (acre- } \\
\text { ft/acre) }\end{array}$ & $\begin{array}{c}\text { Total acres } \\
\text { irrigated }\end{array}$ & $\begin{array}{l}\text { Water used } \\
\text { (acre-ft) }\end{array}$ & $\begin{array}{c}\text { Rate of } \\
\text { irrigation } \\
\text { (acre- } \\
\mathrm{ft} / \text { acre) }\end{array}$ \\
\hline 1991 & 20.86 & $2,748,406$ & $4,213,952$ & 1.53 & $1,255,529$ & $2,036,707$ & 1.62 & $1,267,358$ & $1,830,417$ & 1.44 & NA & NA & $\mathrm{NA}$ \\
\hline 1992 & 25.30 & $2,676,817$ & $3,104,234$ & 1.16 & $1,142,705$ & $1,476,662$ & 1.29 & $1,185,192$ & $1,246,325$ & 1.05 & 125,420 & 119,489 & .95 \\
\hline 1993 & 29.25 & $2,617,957$ & $2,714,695$ & 1.04 & 999,514 & $1,129,571$ & 1.13 & $1,211,003$ & $1,193,480$ & .99 & 148,274 & 128,273 & .87 \\
\hline 1994 & 20.12 & $2,684,893$ & $3,584,044$ & 1.33 & 918,438 & $1,295,570$ & 1.41 & $1,235,635$ & $1,624,275$ & 1.31 & 197,642 & 237,297 & 1.20 \\
\hline 1995 & 23.64 & $2,655,804$ & $3,269,003$ & 1.23 & 771,061 & $1,023,886$ & 1.33 & $1,298,766$ & $1,540,217$ & 1.19 & 216,793 & 243,230 & 1.12 \\
\hline 1996 & 27.21 & $2,672,516$ & $3,023,116$ & 1.13 & 698,963 & 861,699 & 1.23 & $1,351,933$ & $1,457,178$ & 1.08 & 261,523 & 279,077 & 1.07 \\
\hline 1997 & 25.39 & $2,750,671$ & $2,909,044$ & 1.06 & 582,090 & 648,983 & 1.11 & $1,007,008$ & $1,040,186$ & 1.03 & 777,001 & 821,128 & 1.06 \\
\hline 1998 & 23.25 & $2,768,242$ & $3,152,441$ & 1.14 & 469,674 & 513,911 & 1.09 & 889,192 & $1,040,886$ & 1.18 & 966,859 & $1,108,274$ & 1.15 \\
\hline 1999 & 23.92 & $2,792,841$ & $2,859,311$ & 1.02 & 412,421 & 422,260 & 1.02 & 790,129 & 824,782 & 1.04 & $1,131,888$ & $1,159,638$ & 1.02 \\
\hline 2000 & 22.15 & $2,784,282$ & $3,506,969$ & 1.26 & 360,142 & 442,690 & 1.23 & 625,008 & 784,771 & 1.26 & $1,369,081$ & $1,758,993$ & 1.28 \\
\hline 2001 & 20.93 & $2,736,558$ & $3,217,062$ & 1.18 & 297,411 & 334,677 & 1.13 & 533,613 & 630,949 & 1.18 & $1,465,749$ & $1,756,823$ & 1.20 \\
\hline 2002 & 16.89 & $2,798,748$ & $3,842,129$ & 1.37 & 277,431 & 388,052 & 1.40 & 451,492 & 605,786 & 1.34 & $1,568,089$ & $2,176,695$ & 1.39 \\
\hline 2003 & 19.87 & $2,703,979$ & $3,349,440$ & 1.24 & 224,688 & 279,705 & 1.24 & 402,181 & 488,711 & 1.22 & $1,629,760$ & $2,032,483$ & 1.25 \\
\hline Average & 22.98 & $2,722,440$ & $3,288,111$ & 1.21 & 646,928 & 834,952 & 1.25 & 942,193 & $1,101,074$ & 1.18 & 821,507 & 985,117 & 1.13 \\
\hline Mininum & 16.89 & $2,617,957$ & $2,714,695$ & 1.02 & 224,688 & 279,705 & 1.02 & 402,181 & 488,711 & .99 & 125,420 & 119,489 & .87 \\
\hline Maximum & 29.25 & $2,798,748$ & $4,213,952$ & 1.53 & $1,255,529$ & $2,036,707$ & 1.62 & $1,351,933$ & $1,830,417$ & 1.44 & $1,629,760$ & $2,176,695$ & 1.39 \\
\hline Median & 23.25 & $2,736,558$ & $3,217,062$ & 1.18 & 582,090 & 648,983 & 1.23 & $1,007,008$ & $1,046,886$ & 1.18 & 871,930 & 964,701 & 1.13 \\
\hline Slope & -.54 & 8,311 & 17,038 & .001 & $-88,818$ & $-115,553$ & -.018 & $-84,051$ & 100,498 & .007 & 161,420 & 207,285 & .028 \\
\hline$p$-value & .080 & .059 & .760 & .951 & 0 & 0 & .200 & .001 & 0 & .583 & 0 & 0 & .033 \\
\hline
\end{tabular}


Table 12. Trends in precipitation and irrigation by system type, crop type, and prorated crop type for all Groundwater Management Districts, Kansas, 1991-2003.-Continued

Data from Kansas Water Office and Kansas Department of Agriculture (1993-2005). acre-ft, acre-feet; acre-ft/acre, acre-foot per acre. Blue shading indicates trend is significant at 95-percent level (p $\leq 0.05$ ). Green shading indicates trend is significant at 90 -percent level $(\mathrm{p} \leq 0.10)$. NA, data not available]

\begin{tabular}{|c|c|c|c|c|c|c|c|c|c|c|c|c|c|c|c|}
\hline \multirow[b]{2}{*}{ Year } & \multicolumn{3}{|c|}{ Alfalfa } & \multicolumn{3}{|c|}{ Corn } & \multicolumn{3}{|c|}{ Grain sorghum } & \multicolumn{3}{|c|}{ Soybeans } & \multicolumn{3}{|c|}{ Wheat } \\
\hline & $\begin{array}{c}\text { Total } \\
\text { acres } \\
\text { irrigated }\end{array}$ & $\begin{array}{l}\text { Irrigation } \\
\text { water } \\
\text { used } \\
\text { (acre-ft) }\end{array}$ & $\begin{array}{c}\text { Rate of } \\
\text { irri- } \\
\text { gation } \\
\text { (acre- } \\
\mathrm{ft} / \text { acre) }\end{array}$ & $\begin{array}{c}\text { Total } \\
\text { acres } \\
\text { irrigated }\end{array}$ & $\begin{array}{c}\text { Irrigation } \\
\text { water used } \\
\text { (acre-ft) }\end{array}$ & $\begin{array}{c}\text { Rate of } \\
\text { irriga- } \\
\text { tion } \\
\text { (acre- } \\
\text { ft/acre) }\end{array}$ & $\begin{array}{l}\text { Total acres } \\
\text { irrigated }\end{array}$ & $\begin{array}{l}\text { Irrigation } \\
\text { water used } \\
\text { (acre-ft) }\end{array}$ & $\begin{array}{c}\text { Rate of } \\
\text { irriga- } \\
\text { tion } \\
\text { (acre- } \\
\text { ft/acre) }\end{array}$ & $\begin{array}{c}\text { Total acres } \\
\text { irrigated }\end{array}$ & $\begin{array}{l}\text { Irrigation } \\
\text { water used } \\
\text { (acre-ft) }\end{array}$ & $\begin{array}{c}\text { Rate of } \\
\text { irriga- } \\
\text { tion } \\
\text { (acre- } \\
\text { ft/acre) }\end{array}$ & $\begin{array}{l}\text { Total acres } \\
\text { irrigated }\end{array}$ & $\begin{array}{l}\text { Irrigation } \\
\text { water used } \\
\text { (acre-ft) }\end{array}$ & $\begin{array}{c}\text { Rate of } \\
\text { irriga- } \\
\text { tion } \\
\text { (acre- } \\
\mathrm{ft} / \text { acre) }\end{array}$ \\
\hline 1991 & 170,553 & 310,931 & 1.82 & 549,403 & 894,533 & 1.63 & 114,186 & 146,134 & 1.28 & 58,157 & 77,177 & 1.33 & 121,866 & 119,026 & 0.98 \\
\hline 1992 & 156,936 & 223,463 & 1.42 & 605,668 & 682,696 & 1.13 & 111,150 & 86,009 & .77 & 53,484 & 42,258 & .79 & 118,010 & 103,199 & .87 \\
\hline 1993 & 153,921 & 198,053 & 1.29 & 688,231 & 768,144 & 1.12 & 60,315 & 39,611 & .66 & 47,509 & 37,551 & .79 & 102,871 & 69,599 & .68 \\
\hline 1994 & 160,389 & 266,508 & 1.66 & 715,510 & $1,044,370$ & 1.46 & 53,262 & 50,113 & .94 & 56,255 & 68,696 & 1.22 & 83,956 & 71,963 & .86 \\
\hline 1995 & 165,179 & 242,499 & 1.47 & 697,556 & 955,785 & 1.37 & 59,004 & 50,321 & .85 & 52,216 & 55,170 & 1.06 & 85,703 & 57,835 & .67 \\
\hline 1996 & 157,808 & 209,153 & 1.33 & 6,376 & 21,810 & 1 & 866 & 40 & .72 & 4,167 & 3,461 & .80 & 62,576 & 42,927 & .69 \\
\hline 1997 & 174,131 & 243,692 & 1.40 & 899,556 & $1,039,558$ & 1.16 & 46,486 & 32,729 & .70 & 90,284 & 72,571 & .80 & 70,123 & 46,433 & .66 \\
\hline 1998 & 186,439 & 290,103 & 1.56 & 930,388 & $1,193,245$ & 1.28 & 27,873 & 19,923 & .71 & 114,223 & 127,893 & 1.12 & 55,998 & 37,330 & .67 \\
\hline 1999 & 180,020 & 231,132 & 1.28 & 935,436 & $1,085,888$ & 1.16 & 27,607 & 20,314 & .74 & 136,151 & 136,248 & 1.00 & 65,230 & 36,112 & .55 \\
\hline 2000 & 163,139 & 271,837 & 1.67 & 973,269 & $1,382,066$ & 1.42 & 23,342 & 19,732 & .85 & 148,860 & 183,880 & 1.24 & 61,524 & 42,637 & .69 \\
\hline 2001 & 190,008 & 296,017 & 1.56 & 879,634 & $1,178,869$ & 1.34 & 33,189 & 28,601 & .86 & 151,505 & 193,889 & 1.28 & 81,909 & 52,927 & .65 \\
\hline 2002 & 214,420 & 372,587 & 1.74 & 846,089 & $1,316,444$ & 1.56 & 38,944 & 38,317 & .98 & 143,254 & 188,816 & 1.32 & 64,639 & 49,892 & .77 \\
\hline 2003 & 214,826 & 335,361 & 1.56 & 734,453 & $1,064,812$ & 1.45 & 62,598 & 58,164 & .93 & 127,090 & 166,273 & 1.31 & 94,586 & 63,725 & .67 \\
\hline Average & 175,982 & 268,564 & 1.52 & 791,659 & $1,048,325$ & 1.33 & 54,909 & 48,462 & .85 & 94,858 & 107,222 & 1.08 & 82,230 & 61,047 & .72 \\
\hline Mininum & 153,921 & 198,053 & 1.28 & 549,403 & 682,696 & 1.12 & 23,342 & 19,732 & .66 & 47,509 & 37,551 & .79 & 55,998 & 36,112 & .55 \\
\hline Maximum & 214,826 & 372,587 & 1.82 & 973,269 & $1,382,066$ & 1.63 & 114,186 & 146,134 & 1.28 & 151,505 & 193,889 & 1.33 & 121,866 & 119,026 & .98 \\
\hline Median & 170,553 & 266,508 & 1.56 & 836,376 & $1,044,370$ & 1.34 & 53,262 & 39,611 & .85 & 90,284 & 77,177 & 1.12 & 81,909 & 52,927 & .68 \\
\hline Slope & 4,290 & 8,490 & .012 & 30,535 & 40,121 & .011 & $-4,474$ & $-4,134$ & .01 & 9,913 & 13,699 & .036 & $-3,800$ & $-4,230$ & -.001 \\
\hline p-value & .003 & .044 & .502 & .0087 & .003 & .360 & .017 & .059 & .502 & .004 & .003 & .044 & .044 & .044 & .059 \\
\hline
\end{tabular}


Table 12. Trends in precipitation and irrigation by system type, crop type, and prorated crop type for all Groundwater Management Districts, Kansas, 1991-2003.-Continued

Data from Kansas Water Office and Kansas Department of Agriculture (1993-2005). acre-ft, acre-feet; acre-ft/acre, acre-foot per acre. Blue shading indicates trend is significant at 95-percent level $(\mathrm{p} \leq 0.05)$. Green shading indicates trend is significant at 90 -percent level $(\mathrm{p} \leq 0.10)$. NA data not available]

\begin{tabular}{|c|c|c|c|c|c|c|c|c|c|}
\hline \multirow[b]{2}{*}{ Year } & \multicolumn{3}{|c|}{ Other/multiple use } & \multicolumn{6}{|c|}{ Total acres irrigated } \\
\hline & $\begin{array}{c}\text { Totalacres } \\
\text { irrigated }\end{array}$ & $\begin{array}{l}\text { Irrigation } \\
\text { water used } \\
\text { (acre-ft) }\end{array}$ & $\begin{array}{c}\text { Rate of } \\
\text { irrigation } \\
\text { (acre- } \\
\text { ft/acre) }\end{array}$ & $\begin{array}{c}\text { Prorated } \\
\text { alfalfa }\end{array}$ & $\begin{array}{l}\text { Prorated } \\
\text { corn }\end{array}$ & $\begin{array}{l}\text { Prorated } \\
\text { grain } \\
\text { sorghum }\end{array}$ & $\begin{array}{l}\text { Prorated } \\
\text { soybeans }\end{array}$ & $\begin{array}{c}\text { Prorated } \\
\text { wheat }\end{array}$ & $\begin{array}{c}\text { Other } \\
\text { prorated, } \\
\text { unspecified }\end{array}$ \\
\hline 1991 & $1,734,241$ & $2,666,151$ & 1.54 & 186,354 & $1,061,028$ & 389,972 & 58,157 & 601,890 & 86,445 \\
\hline 1992 & $1,631,569$ & $1,966,608$ & 1.21 & 223,116 & $1,121,568$ & 354,829 & 172,372 & 670,197 & 127,703 \\
\hline 1993 & $1,565,110$ & $1,601,733$ & 1.02 & 217,597 & $1,252,824$ & 226,134 & 155,547 & 654,467 & 109,140 \\
\hline 1994 & $1,615,521$ & $2,082,394$ & 1.29 & 227,274 & $1,302,330$ & 219,561 & 160,913 & 649,958 & 124,858 \\
\hline 1995 & $1,596,146$ & $1,907,393$ & 1.19 & 234,234 & $1,272,904$ & 212,908 & 151,655 & 638,298 & 145,157 \\
\hline 1996 & $1,505,723$ & $1,665,727$ & 1.11 & 229,416 & $1,392,529$ & 226,680 & 140,840 & 573,679 & 108,976 \\
\hline 1997 & $1,470,091$ & $1,474,061$ & 1.00 & 246,452 & $1,460,226$ & 180,249 & 184,286 & 586,722 & 91,637 \\
\hline 1998 & $1,453,321$ & $1,483,949$ & 1.02 & 266,184 & $1,472,006$ & 132,311 & 233,699 & 531,110 & 132,932 \\
\hline 1999 & $1,448,397$ & $1,349,617$ & .93 & 251,984 & $1,468,261$ & 127,502 & 267,110 & 522,827 & 155,157 \\
\hline 2000 & $1,414,148$ & $1,606,815$ & 1.14 & 228,385 & $1,501,225$ & 107,393 & 294,190 & 512,553 & 140,539 \\
\hline 2001 & $1,400,313$ & $1,466,756$ & 1.05 & 250,874 & $1,363,507$ & 133,145 & 304,186 & 511,286 & 173,503 \\
\hline 2002 & $1,491,402$ & $1,876,072$ & 1.26 & 288,799 & $1,349,382$ & 174,813 & 283,782 & 530,380 & 171,593 \\
\hline 2003 & $1,470,426$ & $1,661,107$ & 1.13 & 291,541 & $1,188,203$ & 218,536 & 255,954 & 551,252 & 198,494 \\
\hline Average & $1,522,801$ & $1,754,491$ & 1.14 & 241,708 & $1,323,538$ & 208,003 & 204,822 & 579,586 & 135,856 \\
\hline Mininum & $1,400,313$ & $1,349,617$ & .93 & 186,354 & $1,061,028$ & 107,393 & 58,157 & 511,286 & 86,445 \\
\hline Maximum & $1,734,241$ & $2,666,151$ & 1.54 & 291,541 & $1,501,225$ & 389,972 & 304,186 & 670,197 & 198,494 \\
\hline Median & $1,491,402$ & $1,661,107$ & 1.13 & 234,234 & $1,349,382$ & 212,908 & 184,286 & 573,679 & 132,932 \\
\hline Slope & $-21,324$ & $-45,891$ & -.013 & 6,764 & 26,548 & $-16,384$ & 16,509 & $-11,117$ & 6,944 \\
\hline $\mathrm{p}$-value & .0012 & .059 & .2463 & .001 & .059 & .0087 & .0041 & .002 & .004 \\
\hline
\end{tabular}


Table 13. Results of regression analysis for irrigation water use and irrigation application rates based on average annual county precipitation for 1991-2003.

[Shading indicates correlation coefficients $(\mathrm{R})$ greater than or equal to 0.71 , explaining greater than 50 percent of the variability. $x$ equals average annual precipitation, in inches; --, not determined]

\begin{tabular}{|c|c|c|c|c|}
\hline $\begin{array}{l}\text { County } \\
\text { (fig. 1) }\end{array}$ & Water-use equation & $\begin{array}{l}\text { Water-use } \\
\text { correlation } \\
\text { coefficient } \\
\text { (R) }\end{array}$ & Application rate equation & $\begin{array}{l}\text { Application } \\
\text { rate } \\
\text { correlation } \\
\text { coefficient }(\mathrm{R})\end{array}$ \\
\hline$\overline{\text { Barton }}$ & $y=-1501 x+69062$ & 0.81 & $y=-0.0123 x+1.664$ & 0.12 \\
\hline Cheyenne & $y=-1339.7 x+73702$ & .54 & $y=-0.0282 x+1.7491$ & .18 \\
\hline Edwards & $y=-3361.9 x+189271$ & .83 & $y=-0.0097 x+1.6119$ & .08 \\
\hline Finney & $y=-9630.4 x+489486$ & .68 & $y=-0.0036 x+1.3451$ & 0 \\
\hline Ford & $y=-2836.8 x+155916$ & .85 & $y=-0.0032 x+1.402$ & .01 \\
\hline Graham & $y=-535.24 x+21700$ & .79 & $y=-0.0154 x+1.6538$ & .14 \\
\hline Grant & $y=-1648.3 x+194630$ & .17 & $y=0.0246 x+0.9566$ & .05 \\
\hline Gray & $y=-3824.1 x+315811$ & .57 & $y=-0.0077 x+1.4623$ & .04 \\
\hline Greeley & $y=-1130.3 x+47359$ & .43 & $y=-0.0441 x+2.093$ & .24 \\
\hline Harvey & $y=-460.87 x+40082$ & .35 & $y=-0.0067 x+1.6175$ & .01 \\
\hline Haskell & $y=-3864.1 x+350668$ & .33 & $y=0.0037 x+1.272$ & 0 \\
\hline Kearny & $y=-3208.9 x+199603$ & .64 & $y=-0.036 x+1.9801$ & .24 \\
\hline Kiowa & $y=-1495.4 x+93668$ & .70 & $y=0.0084 x+1.1972$ & .05 \\
\hline Lane & $y=-363.8 x+25214$ & .39 & $y=-0.0121 x+1.5662$ & .06 \\
\hline McPherson & $y=-913.32 x+52321$ & .68 & $y=-0.0082 x+1.6353$ & .06 \\
\hline Meade & $y=-1620.7 x+205089$ & .41 & $y=0.0412 x+0.6042$ & .29 \\
\hline Morton & $y=-1174.2 x+76901$ & .22 & $y=-0.0007 x+1.406$ & 0 \\
\hline Pawnee & $y=-3251.9 x+141463$ & .91 & $y=-0.0034 x+1.439$ & .02 \\
\hline Pratt & $y=-2161.9 x+138174$ & .62 & $y=-0.0056 x+1.5667$ & .01 \\
\hline Rawlins & $y=-955.26 x+34492$ & .85 & $y=-0.0463 x+2.1954$ & .44 \\
\hline Reno & $y=-56.154 x+39110$ & .07 & $y=0.0026 x+1.3713$ & 0 \\
\hline Rice & $y=-907.33 x+42439$ & .82 & $y=0.0006 x+1.3702$ & 0 \\
\hline Scott & $y=-1533.8 x+88164$ & .40 & $y=-0.0181 x+1.626$ & .06 \\
\hline Sedgwick & $y=-688.53 x+54393$ & .50 & $y=-0.0018 x+1.4901$ & 0 \\
\hline Seward & $y=-2061.3 x+201174$ & .36 & $y=0.0875 x-0.082$ & .44 \\
\hline Sheridan & $y=-3264.9 x+141063$ & .83 & $y=-0.021 x+1.7417$ & .26 \\
\hline Sherman & $y=-4164 x+192872$ & .78 & $y=-0.03 x+1.7905$ & .32 \\
\hline Stafford & $y=-2371.9 x+141578$ & .71 & $y=-0.005 x+1.5$ & .02 \\
\hline Stanton & $y=-3520.1 x+225817$ & .30 & $y=-0.0022 x+1.3796$ & 0 \\
\hline Stevens & $y=-2528.7 x+258922$ & .40 & $y=0.0064 x+1.3172$ & 0 \\
\hline Thomas & $y=-5184.5 x+201931$ & .86 & $y=-0.0485 x+2.2238$ & .51 \\
\hline Wallace & $y=-1584.5 x+99184$ & .54 & $y=-0.0331 x+1.8789$ & .22 \\
\hline Wichita & $y=-3500.3 x+142917$ & .66 & $y=-0.001 x+1.3282$ & 0 \\
\hline $\begin{array}{l}\text { Average for all } \\
\text { counties }\end{array}$ & -- & .58 & -- & .12 \\
\hline
\end{tabular}


Table 14. Results of regression analysis for irrigation water use based on average annual precipitation by meteorological region for 1991-2003.

\begin{tabular}{cllc}
\hline $\begin{array}{c}\text { Groundwater } \\
\begin{array}{c}\text { Management } \\
\text { District } \\
\text { number } \\
\text { (fig. 1) }\end{array}\end{array}$ & Water-use equation & \multicolumn{1}{c}{ Meteorological region (fig. 1) } & $\begin{array}{c}\text { Percent } \\
\text { variability } \\
\text { explained } \\
\left(\mathrm{R}^{2}\right) \mathrm{x} 100\end{array}$ \\
\hline 1 & $\mathrm{y}=-4,720 \mathrm{x}+354,000$ & $\mathrm{x}=$ Region 4 average annual precipitation & 20 \\
2 & $\mathrm{y}=-3,670 \mathrm{x}+207,000$ & $\mathrm{x}=$ Region 8 average annual precipitation & 34 \\
3 & $\mathrm{y}=-43,200 \mathrm{x}+2,930,000$ & $\mathrm{x}=$ Region 7 average annual precipitation & 31 \\
4 & $\mathrm{y}=-13,400 \mathrm{x}+676,000$ & $\mathrm{x}=$ Region 1 average annual precipitation & 68 \\
5 & $\mathrm{y}=-19,800 \mathrm{x}+1,070,000$ & $\mathrm{x}=$ Region 8 average annual precipitation & 59 \\
All & $\mathrm{y}=-96,300+5,500,000$ & $\mathrm{x}=$ Average of regions 1, 4, 7, and 8 & 60 \\
& & precipitation & \\
\hline
\end{tabular}


Table 15. Irrigation water use adjusted using average regional precipitation by meteorological region for Groundwater Management Districts within the Kansas High Plains, 1991-2003.

\begin{tabular}{|c|c|c|c|c|}
\hline Year & $\begin{array}{c}\text { Average } \\
\text { precipitation for } \\
\text { meteorological } \\
\text { region } \\
1,4,7 \text {, and } 8 \\
\text { (fig. } 1 \text { ) (inches) }\end{array}$ & $\begin{array}{l}\text { Total irrigation } \\
\text { water use (acre- } \\
\text { feet) }\end{array}$ & $\begin{array}{l}\text { Regression- } \\
\text { equation water } \\
\text { use, equation } 1^{1} \\
\text { (acre-feet) }\end{array}$ & $\begin{array}{l}\text { Precipitation- } \\
\text { adjusted water } \\
\text { use, equation } 2^{2} \\
\text { (acre-feet) }\end{array}$ \\
\hline 1991 & 20.86 & $4,213,952$ & $3,492,000$ & $3,824,000$ \\
\hline 1992 & 25.30 & $3,104,234$ & $3,064,000$ & $3,418,000$ \\
\hline 1993 & 29.25 & $2,714,695$ & $2,683,000$ & $3,455,000$ \\
\hline 1994 & 20.12 & $3,584,044$ & $3,563,000$ & $3,137,000$ \\
\hline 1995 & 23.64 & $3,269,003$ & $3,223,000$ & $3,363,000$ \\
\hline 1996 & 27.21 & $3,023,116$ & $2,880,000$ & $3,580,000$ \\
\hline 1997 & 25.39 & $2,909,044$ & $3,055,000$ & $3,214,000$ \\
\hline 1998 & 23.25 & $3,152,441$ & $3,261,000$ & $3,190,000$ \\
\hline 1999 & 23.92 & $2,859,311$ & $3,196,000$ & $2,976,000$ \\
\hline 2000 & 22.15 & $3,506,969$ & $3,367,000$ & $3,380,000$ \\
\hline 2001 & 20.93 & $3,217,062$ & $3,484,000$ & $2,930,000$ \\
\hline 2002 & 16.89 & $3,842,129$ & $3,874,000$ & $2,823,000$ \\
\hline 2003 & 19.87 & $3,349,440$ & $3,586,000$ & $2,896,000$ \\
\hline Average & 22.98 & $\begin{array}{l}\mathrm{p}=0.760 \\
\text { slope }=+17,000\end{array}$ & $\begin{array}{l}\mathrm{p}=0.076 \\
\text { slope }=+52,100\end{array}$ & $\begin{array}{l}\mathrm{p}=0.0003 \\
\text { slope }=-77,700\end{array}$ \\
\hline
\end{tabular}

${ }^{1}$ Equation 1: Estimated water use $=-96,300 \times($ average regional precipitation $)+5,500,000$.

${ }^{2}$ Equation 2: Estimated water use $=$ Annual water use $\mathrm{x}$ (average regional precipitation/annual regional precipitation). 
Table 16. Average precipitation plus irrigation-applied water for all Groundwater Management Districts within the Kansas High Plains, 1991-2003.

\begin{tabular}{|c|c|c|c|c|}
\hline Year & $\begin{array}{c}\text { Average } \\
\text { precipitation } \\
\text { for } \\
\text { meteorological } \\
\text { regions 1, 4, 7, } \\
\text { and } 8 \text { (fig. 1) } \\
\text { (inches) }\end{array}$ & $\begin{array}{l}\text { All Groundwater } \\
\text { Management } \\
\text { Districts average } \\
\text { irrigation } \\
\text { application rate } \\
\text { (acre-feet per } \\
\text { acre) }\end{array}$ & $\begin{array}{l}\text { All Groundwater } \\
\text { Management } \\
\text { Districts average } \\
\text { irrigation } \\
\text { application rate } \\
\text { (inches) }\end{array}$ & $\begin{array}{c}\text { Average } \\
\text { precipitation plus } \\
\text { irrigation } \\
\text { application rate } \\
\text { (inches) }\end{array}$ \\
\hline 1991 & 20.86 & 1.53 & 18.40 & 39.25 \\
\hline 1992 & 25.30 & 1.16 & 13.92 & 39.22 \\
\hline 1993 & 29.25 & 1.04 & 12.44 & 41.69 \\
\hline 1994 & 20.12 & 1.33 & 16.02 & 36.13 \\
\hline 1995 & 23.64 & 1.23 & 14.77 & 38.41 \\
\hline 1996 & 27.21 & 1.13 & 13.57 & 40.78 \\
\hline 1997 & 25.39 & 1.06 & 12.69 & 38.08 \\
\hline 1998 & 23.25 & 1.14 & 13.67 & 36.92 \\
\hline 1999 & 23.92 & 1.02 & 12.29 & 36.21 \\
\hline 2000 & 22.15 & 1.26 & 15.11 & 37.26 \\
\hline 2001 & 20.93 & 1.18 & 14.11 & 35.04 \\
\hline 2002 & 16.89 & 1.37 & 16.47 & 33.36 \\
\hline 2003 & 19.87 & 1.24 & 14.86 & 34.74 \\
\hline
\end{tabular}


Table 17. Relation between irrigation system types and application rates, 1992-2003, for Groundwater Management Districts within the Kansas High Plains.

\begin{tabular}{|c|c|c|c|c|}
\hline $\begin{array}{c}\text { Groundwater } \\
\text { Management } \\
\text { District number } \\
\text { (fig. 1) }\end{array}$ & $\begin{array}{c}\text { Center pivot } \\
\text { irrigation rate } \\
\text { compared to } \\
\text { drop nozzle } \\
\text { irrigation rate }\end{array}$ & $\begin{array}{l}\text { Percent variability } \\
\text { explained, } R^{2} \times 100 \\
\text { (Y-intercept=0) }\end{array}$ & $\begin{array}{l}\text { Flood irrigation } \\
\text { rate compared } \\
\text { to drop nozzle } \\
\text { irrigation rate }\end{array}$ & $\begin{array}{l}\text { Percent variability } \\
\text { explained, } R^{2} \times 100 \\
(Y \text {-intercept=0) }\end{array}$ \\
\hline 1 & $y=1.048 x$ & 74 & $y=0.9302 x$ & 78 \\
\hline 2 & $y=0.9834 x$ & 70 & $y=0.8206$ & 82 \\
\hline 3 & $y=0.9886 x$ & 69 & $y=0.97 x$ & 14 \\
\hline 4 & $y=1.0004 x$ & 96 & $\mathrm{y}=0.8219 \mathrm{x}$ & 81 \\
\hline 5 & $y=1.0007 x$ & 90 & $y=1.1024 x$ & 57 \\
\hline $\begin{array}{l}\text { All Groundwater } \\
\text { Management } \\
\text { Districts }\end{array}$ & $y=0.9793 x$ & 83 & $y=0.9233 x$ & 12 \\
\hline $\begin{array}{l}\text { All counties in } \\
\text { study area }\end{array}$ & $y=0.9789$ & 62 & $y=0.8885 x$ & 19 \\
\hline
\end{tabular}


Table 18. Correlation coefficients for irrigation water-use factors for 33 counties in the Groundwater Management Districts within the Kansas High Plains, $1991-2003$.

[Shading indicates correlation coefficients greater than or equal to 0.50 . --, not applicable]

\begin{tabular}{|c|c|c|c|c|c|c|c|c|c|c|c|c|c|c|c|c|c|c|c|c|c|c|c|}
\hline & \multirow{2}{*}{$\begin{array}{c}\text { Total } \\
\text { water } \\
\text { used for } \\
\text { irri- } \\
\text { gation }\end{array}$} & \multirow{2}{*}{$\begin{array}{c}\text { Total } \\
\text { acres } \\
\text { irri- } \\
\text { gated }\end{array}$} & \multicolumn{2}{|c|}{ Alfalfa } & \multicolumn{2}{|c|}{ Corn } & \multicolumn{2}{|c|}{ Grain sorghum } & \multicolumn{2}{|c|}{ Soybeans } & \multicolumn{2}{|c|}{ Wheat } & \multicolumn{2}{|c|}{$\begin{array}{l}\text { Other/multiple } \\
\text { uses }\end{array}$} & \multicolumn{2}{|c|}{ Flood irrigation } & \multicolumn{2}{|c|}{$\begin{array}{l}\text { Center pivot } \\
\text { irrigation }\end{array}$} & \multicolumn{2}{|c|}{$\begin{array}{c}\text { Drop nozzle } \\
\text { irrigation }\end{array}$} & \multirow{2}{*}{$\begin{array}{l}\text { Annual } \\
\text { precip- } \\
\text { itation }\end{array}$} & \multirow{2}{*}{$\begin{array}{l}\text { March- } \\
\text { October } \\
\text { precip- } \\
\text { itation }\end{array}$} & \multirow{2}{*}{$\begin{array}{c}\text { County } \\
\text { soil } \\
\text { permea- } \\
\text { bility }\end{array}$} \\
\hline & & & Acres & $\begin{array}{c}\text { Water } \\
\text { appli- } \\
\text { cation } \\
\text { rate }\end{array}$ & Acres & $\begin{array}{c}\text { Water } \\
\text { appli- } \\
\text { cation } \\
\text { rate }\end{array}$ & Acres & $\begin{array}{c}\text { Water } \\
\text { appli- } \\
\text { cation } \\
\text { rate }\end{array}$ & Acres & $\begin{array}{c}\text { Water } \\
\text { appli- } \\
\text { cation } \\
\text { rate }\end{array}$ & Acres & $\begin{array}{c}\text { Water } \\
\text { appli- } \\
\text { cation } \\
\text { rate }\end{array}$ & Acres & $\begin{array}{c}\text { Water } \\
\text { appli- } \\
\text { cation } \\
\text { rate }\end{array}$ & Acres & $\begin{array}{c}\text { Water } \\
\text { appli- } \\
\text { cation } \\
\text { rate }\end{array}$ & Acres & $\begin{array}{c}\text { Water } \\
\text { appli- } \\
\text { cation } \\
\text { rate }\end{array}$ & Acres & $\begin{array}{l}\text { Water } \\
\text { appli- } \\
\text { cation } \\
\text { rate }\end{array}$ & & & \\
\hline $\begin{array}{l}\text { Total acre-feet of } \\
\text { water }\end{array}$ & 1 & -- & -- & -- & -- & -- & -- & -- & -- & -- & -- & -- & -- & -- & -- & -- & -- & -- & -- & -- & -- & -- & -- \\
\hline $\begin{array}{c}\text { Total acres } \\
\text { irrigated }\end{array}$ & .97 & 1 & -- & -- & -- & -- & -- & -- & -- & -- & -- & -- & -- & -- & -- & -- & -- & -- & -- & -- & -- & -- & -- \\
\hline Alfalfa acres & .61 & .63 & 1 & -- & -- & -- & -- & -- & -- & -- & -- & -- & -- & -- & -- & -- & -- & -- & -- & -- & -- & -- & -- \\
\hline $\begin{array}{l}\text { Alfalfa water } \\
\text { application rate }\end{array}$ & .55 & .48 & .33 & 1 & -- & -- & -- & -- & -- & -- & -- & -- & -- & -- & -- & -- & -- & -- & -- & -- & -- & -- & -- \\
\hline Corn acres & .58 & .66 & .44 & .19 & 1 & -- & -- & -- & -- & -- & -- & -- & -- & -- & -- & -- & -- & -- & -- & -- & -- & -- & -- \\
\hline $\begin{array}{l}\text { Corn water } \\
\text { application rate }\end{array}$ & .70 & .58 & .31 & .70 & .20 & 1 & -- & -- & -- & -- & -- & -- & -- & -- & -- & -- & -- & -- & -- & -- & -- & -- & -- \\
\hline $\begin{array}{l}\text { Grain sorghum } \\
\text { acres }\end{array}$ & .18 & .21 & .24 & .10 & .06 & .05 & 1 & -- & -- & -- & -- & -- & -- & -- & -- & -- & -- & -- & -- & -- & -- & -- & -- \\
\hline $\begin{array}{l}\text { Grain sorghum } \\
\text { water } \\
\text { application rate }\end{array}$ & .33 & .22 & .14 & .38 & -.01 & .55 & .15 & 1 & -- & -- & -- & -- & -- & -- & -- & -- & -- & -- & -- & -- & -- & -- & -- \\
\hline Soybean acres & -.09 & -.06 & .12 & -.26 & .22 & -.25 & .01 & -.03 & 1 & -- & -- & -- & -- & -- & -- & -- & -- & -- & -- & -- & -- & -- & -- \\
\hline $\begin{array}{l}\text { Soybean water } \\
\text { application rate }\end{array}$ & .23 & .21 & .27 & .10 & .36 & .19 & .11 & .10 & .33 & 1 & -- & -- & -- & -- & -- & -- & -- & -- & -- & -- & -- & -- & -- \\
\hline Wheat acres & .69 & .72 & .67 & .37 & .43 & .39 & .42 & .17 & -.07 & .16 & 1 & -- & -- & -- & -- & -- & -- & -- & -- & -- & -- & -- & -- \\
\hline $\begin{array}{l}\text { Wheat water } \\
\text { application rate }\end{array}$ & .50 & .41 & .22 & .48 & .05 & .62 & .16 & .43 & -.24 & -.03 & .32 & 1 & -- & -- & -- & -- & -- & -- & -- & -- & -- & -- & -- \\
\hline $\begin{array}{l}\text { Other/multiple use } \\
\text { acres }\end{array}$ & .91 & .90 & .39 & .51 & .34 & .64 & .13 & .26 & -.29 & .03 & .58 & .49 & 1 & -- & -- & -- & -- & -- & -- & -- & -- & -- & -- \\
\hline $\begin{array}{l}\text { Other/multiple use } \\
\text { water } \\
\text { application rate }\end{array}$ & .58 & .42 & .15 & .58 & .10 & .86 & .08 & .62 & -.07 & .22 & .23 & .57 & .49 & 1 & -- & -- & -- & -- & -- & -- & -- & -- & -- \\
\hline $\begin{array}{l}\text { Flood irrigation } \\
\text { acres }\end{array}$ & .66 & .61 & .26 & .32 & .04 & .47 & .29 & .30 & -.29 & -.05 & .50 & .49 & .75 & .43 & 1 & - & -- & -- & -- & -- & -- & -- & -- \\
\hline $\begin{array}{l}\text { Flood irrigation } \\
\text { water } \\
\text { application rate }\end{array}$ & .48 & .35 & .05 & .45 & .22 & .75 & .01 & .46 & -.13 & .19 & .21 & .44 & .37 & .80 & .25 & 1 & -- & -- & -- & -- & -- & -- & -- \\
\hline $\begin{array}{l}\text { Center pivot } \\
\text { irrigation acres }\end{array}$ & .60 & .65 & .59 & .26 & .59 & .25 & .36 & .13 & .10 & .13 & .65 & .18 & .44 & .21 & .32 & .18 & 1 & -- & -- & -- & -- & -- & -- \\
\hline $\begin{array}{l}\text { Center pivot } \\
\text { irrigation water } \\
\text { application rate }\end{array}$ & .64 & .51 & .43 & .68 & .28 & .84 & .02 & .54 & -.06 & .29 & .33 & .54 & .47 & .82 & .34 & .59 & .31 & 1 & -- & -- & -- & -- & -- \\
\hline $\begin{array}{l}\text { Drop nozzle } \\
\text { irrigation acres }\end{array}$ & .48 & .53 & .44 & .27 & .59 & .27 & -.12 & -.01 & .22 & .34 & .25 & .07 & .35 & .11 & -.14 & .14 & .01 & .28 & 1 & -- & -- & -- & -- \\
\hline $\begin{array}{l}\text { Drop nozzle } \\
\text { irrigation water } \\
\text { application rates }\end{array}$ & .30 & .31 & .28 & .24 & .33 & .26 & -.28 & -.06 & .07 & .11 & .09 & .07 & .23 & .13 & -.07 & .10 & .07 & .32 & .49 & 1 & -- & -- & -- \\
\hline $\begin{array}{l}\text { Annual } \\
\text { precipitation }\end{array}$ & -.39 & -.32 & -.14 & -.59 & -.12 & -.67 & .05 & -.37 & .40 & -.10 & -.22 & -.43 & -.39 & -.53 & -.19 & -.55 & .05 & -.56 & -.33 & -.28 & 1 & -- & -- \\
\hline $\begin{array}{l}\text { March-October } \\
\text { precipitation }\end{array}$ & -.37 & -.29 & -.12 & -.58 & -.08 & -.64 & .02 & -.38 & .37 & -.11 & -.21 & -.42 & -.36 & -.54 & -.21 & -.54 & .06 & -.54 & -.28 & -.23 & .97 & 1 & -- \\
\hline $\begin{array}{l}\text { County soil } \\
\text { permeability }\end{array}$ & -.12 & -.01 & -.04 & -.29 & .04 & -.39 & .01 & -.34 & -.07 & -.30 & -.01 & -.19 & -.02 & -.46 & .01 & -.42 & .14 & -.39 & -.07 & -.13 & .20 & .18 & 1 \\
\hline
\end{tabular}


Table 19. Predictive equations for irrigation water use and irrigation application rates from county data for all Groundwater Management Districts within the Kansas High Plains, 1991-2003.

[TWU, irrigation water use, in acre-feet; TA, total acres irrigated; MOP, March-October precipitation by county, in inches; AP, annual precipitation by county, in inches; CR, water application rate for corn, in acre-feet per acre; AR, water application rate for alfalfa, in acre-feet per acre; WR, water application rate for wheat, in acre-feet per acre; OA, other/multiple-use acres; SP, soil permeability]

\begin{tabular}{|c|c|c|c|c|}
\hline Predictor equation & $\begin{array}{c}\text { Percent } \\
\text { variability } \\
\text { explained } \\
\left(R^{2}\right) \times 100\end{array}$ & $\begin{array}{c}\text { Residual standard } \\
\text { error }^{1} \\
\text { (acre-feet) }\end{array}$ & $\begin{array}{l}\text { Degree of } \\
\text { freedom }\end{array}$ & F statistic ${ }^{2}$ \\
\hline TWU $=1.3809(\mathrm{TA})-14,866$ & 94 & 19,990 & 427 & 6,573 \\
\hline TWU $=-4,826(\mathrm{AP})+210,202$ & 16 & 74,410 & 427 & 78 \\
\hline $\mathrm{TWU}=-5,351(\mathrm{MOP})+206,781$ & 14 & 75,120 & 427 & 68.8 \\
\hline $\mathrm{TWU}=1.3385(\mathrm{TA})-1140(\mathrm{AP})+14,811$ & 95 & 18,700 & 426 & 3,788 \\
\hline $\mathrm{TWU}=1.3402(\mathrm{TA})-1,400(\mathrm{MOP})+16,576$ & 95 & 18,530 & 426 & 3,859 \\
\hline TWU $=184,305(\mathrm{CR})-135,625$ & 49 & 57,560 & 427 & 145 \\
\hline $\mathrm{TWU}=17,310(\mathrm{AR})+149,728(\mathrm{CR})+26,504(\mathrm{WR})-129,328$ & 51 & 57,010 & 425 & 417 \\
\hline $\mathrm{TWU}=1.7917(\mathrm{OA})+16.698$ & 82 & 34,090 & 427 & 1,980 \\
\hline $\mathrm{CR}=-0.0311(\mathrm{AP})+1.999$ & 43 & .2304 & 427 & 339.6 \\
\hline $\mathrm{CR}=-0.0333(\mathrm{AP})+0.0575(\mathrm{SP})+1.9131$ & 49 & .2202 & 426 & 206.4 \\
\hline $\mathrm{AR}=-0.0253(\mathrm{AP})+0.0641(\mathrm{SP})+1.5530$ & 45 & .188 & 426 & 173 \\
\hline
\end{tabular}

${ }^{1}$ Residual standard error $=$ standard deviation of difference between predicted value and actual value. Lower number defines a better model.

${ }^{2} \mathrm{~F}$-statistic $=$ a test to see if the model coefficients are statistically different from zero. A higher number defines a better model. 